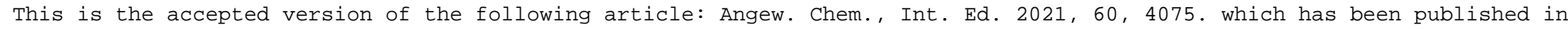
final form at https://onlinelibrary.wiley.com/doi/10.1002/anie.202013516. This article may be used for non-commercial purposes in accordance With Wiley-VCH Terms and Conditions for self-archiving

\title{
Azide Radical Initiated Ring Opening of Cyclopropenes Leading to Alkenyl Nitriles and Polycyclic Aromatic Compounds
}

\author{
Bastian Muriel and Jerome Waser ${ }^{*[a]}$
}

\begin{abstract}
We report herein a radical-mediated amination of cyclopropenes. The transformation proceeds through a cleavage of the three-membered ring after the addition of an azide radical on the strained double bond and leads to tetrasubstituted alkenyl nitrile derivatives upon loss of $\mathrm{N}_{2}$. With 1,2-diaryl substituted cyclopropenes, this methodology could be extended to a one pot synthesis of highly functionalized polycyclic aromatic compounds (PACs). This transformation allows the synthesis of nitrile-substituted alkenes and aromatic compounds from rapidly accessed cyclopropenes using only commercially available reagents.
\end{abstract}

Alkenyl nitriles and polycyclic aromatic compounds (PACs) are important scaffolds in organic chemistry, material sciences and medicine. ${ }^{1]}$ Alkenyl nitriles are key structural motifs in pharmaceuticals such as Entacapone used in the treatment of Parkinson's disease, ${ }^{[2]}$ or the reverse transcriptase inhibitor Rilpivirine. ${ }^{[3]}$ In addition, they are valuable synthetic intermediates that have been used as electrophilic partners in conjugate additions. ${ }^{[4]}$ PACs are also present in biologically active compounds, ${ }^{[5]}$ and have found many applications as organic materials for semiconductors ${ }^{[6]}$ and light-emitting diodes. ${ }^{[7]}$. For these reasons, developing new methods for the synthesis of PACs has been the focus of intensive research. ${ }^{\left[{ }^{[]}\right.}$Nevertheless, there is still a strong demand for modular methods to access selectively polysubstituted PACs.

Cyclopropenes have emerged in the past decades as important three-carbon building blocks, due to efficient methods for their synthesis combined with a versatile reactivity. ${ }^{[9]}$ Reaction at the double bond with organometallic intermediates or transition metal catalysts gives access to either functionalized cyclopropanes ${ }^{[10]}$ or substituted alkenes after ring-opening via vinyl carbene intermediates ${ }^{[11]}$ (Scheme 1, a). The formation of a reactive cyclopropyl radical intermediate upon the addition of a radical onto the alkene has been less explored. Nakamura and coworkers reported in 1994 the hydrostannation of cyclopropenes. ${ }^{[12]}$ Several reports have later described the addition of carbon-centered radicals, with a functionalization of the resulting cyclopropyl radical through $\mathrm{C}$-S bond formation, ${ }^{[13]}$ $\mathrm{H}$-abstraction, ${ }^{[14]}$ or a second $\mathrm{C}-\mathrm{C}$ bond forming event. ${ }^{[15]}$ We recently developed a photoredox-mediated $(3+2)$ annulation with cyclopropylanilines to access bicyclo[3.1.0]hexanes. ${ }^{[16]}$ In contrast, examples of a radical functionalization of cyclopropenes leading to a cleavage of the three-membered ring are scarce (Scheme 1, b). Miyata and co-workers reported a ring opening rearrangement after the addition of a trichloromethyl radical to

$\left.{ }^{*}\right] \quad$ B. Muriel, Prof. Dr. J. Waser
Laboratory of Catalysis and Organic Synthesis, Ecole Polytechnique Fédérale de Lausanne, EPFL, SB ISIC LCSO, BCH 4306 1015 Lausanne (Switzerland) Email: jerome.waser@epfl.ch Homepage : http://lcso.epfl.ch

Supporting information for this article is given via a link at the end of the document. Raw data for NMR, IR and MS is available at zenodo.org, DOI: 10.5281/zenodo.4279268 alkyl-substituted cyclopropenes, followed by trapping of the radical by dimethyl zinc (Scheme 1, b) 1.). ${ }^{[14 a]}$ Landais and coworkers developed a radical-mediated dicarbofunctionalization of cyclopropenes, followed by a base-mediated fragmentation of the in situ formed cyclopropane (Scheme 1, b) 2.). ${ }^{[17]}$

a) Cyclopropenes for the synthesis of Substituted Cyclopropanes and Alkenes

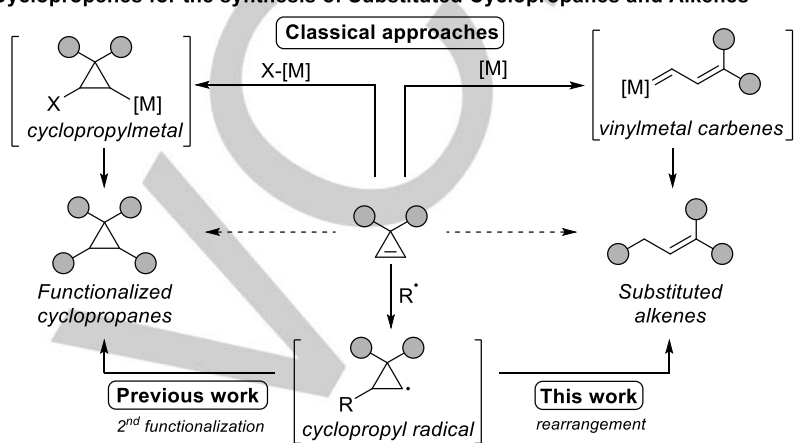

b) Radical Functionalization of Cyclopropenes with Ring Cleavage: Precedents 1. Zn-mediated ring cleavage

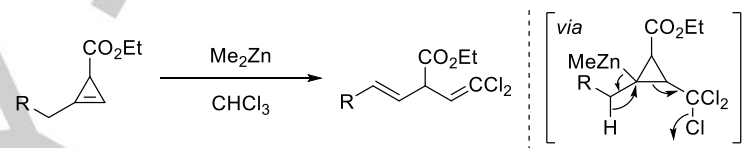

2. in situ base-mediated ring cleavage

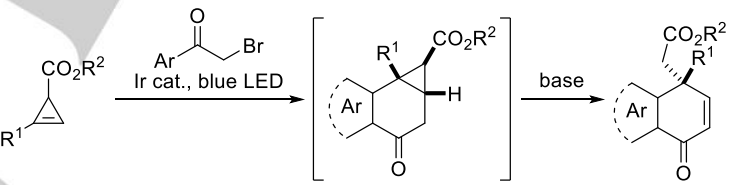

c) This work: Radical Amination of Cyclopropenes to Give Alkenyl Nitriles and PACs

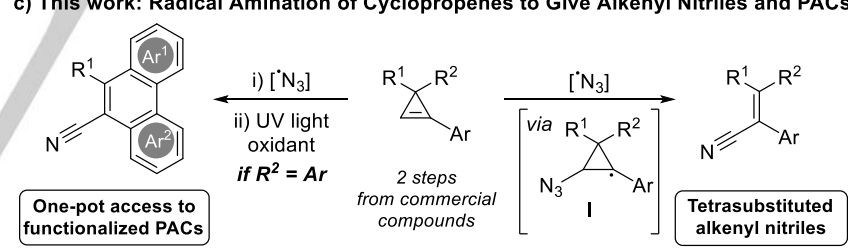

Scheme 1. Synthetic strategies towards functionalized cyclopropanes and substituted alkenes from cyclopropenes (a). Previous reports leading to a cleavage of the three-membered ring after a radical functionalization of cyclopropenes (b). This work: radical amination of cyclopropenes with an azide radical for the synthesis of alkenyl nitriles and substituted PACs (c).

Although examples of amination reactions of cyclopropenes have been reported in the presence of transition metals or lanthanides combined with electrophilic, ${ }^{[18]}$ or nucleophilic sources of nitrogen, ${ }^{[19]}$ the addition of $\mathrm{N}$-centered radicals has not been reported to date. We report herein a ring-opening reaction initiated by the addition of an azide radical (Scheme 1, c). This transformation led to the formation of alkenyl nitriles after the rearrangement of a putative azido-cyclopropyl radical (I). With 1,2-diaryl substituted cyclopropenes we developed a one-pot radical amination / oxidative photocyclization to access highly substituted PACs. While azide radicals have already been used for the synthesis of alkenyl nitriles through $\mathrm{C}-\mathrm{H}$ or $\mathrm{C}-\mathrm{C}$ bond cleavage of alkenes and alkynes, these processes were limited to 
the synthesis of trisubstituted olefins and cyclopropenes were not reported as substrates. ${ }^{[20]}$

Our investigations started with cyclopropene 1 employing ABZ (3) as safe azidation reagent under mild photoredox-catalyzed conditions known to generate azide radicals (Table 1). ${ }^{\left[{ }^{21]}\right.}$ Under green LED irradiation with $\mathrm{Cu}(\mathrm{dap})_{2} \mathrm{Cl}$ as photocatalyst, alkenyl nitrile 2 was formed in $82 \%$ yield (entry 1 ). When reducing the equivalents of $A B Z$ from 2.0 to 1.4, 2 was obtained in $78 \%$ yield (entry 2). In the presence of inexpensive $\mathrm{CuCl}_{2}$ and $\mathrm{ABZ}$ (3) without irradiation, 2 was formed in $62 \%$ yield (entry 3 ). Employing 2 equivalents of PIDA and TMS- $\mathrm{N}_{3}$ as commercial reagents, ${ }^{[22]} 2$ was obtained in $56 \%$ yield after 30 minutes of reaction (entry 4 ). Adding $10 \mathrm{~mol} \%$ of $\mathrm{CuCl}_{2}$ led to a high yield of $94 \%$ (entry 5). ${ }^{[23]}$ While going from 2.0 to 1.5 equivalent led to a decrease in yield to $85 \%$ (entry 6 ), we found 1.8 equivalent to be optimal combined with a reduced loading of $\mathrm{CuCl}_{2}$ to $5 \mathrm{~mol} \%$ (entry 7 ). Under these conditions, 2 could be isolated in $88 \%$ yield.

Table 1. Discovery and optimization of the radical amination. ${ }^{[a]}$

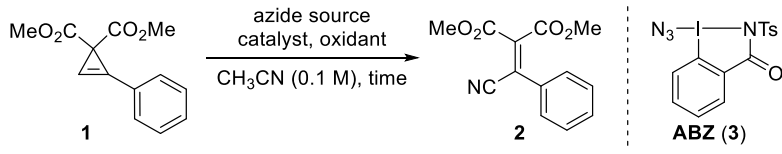

\begin{tabular}{|c|c|c|c|c|c|}
\hline Entry & $\begin{array}{l}\text { Azide Source } \\
\text { (equiv.) }\end{array}$ & $\begin{array}{c}\text { Cat. } \\
\text { (mol\%) }\end{array}$ & $\begin{array}{l}\text { Oxidant } \\
\text { (equiv.) }\end{array}$ & Time & Yield $^{[b]}$ \\
\hline $1^{[c]}$ & $A B Z(2)$ & $\mathrm{Cu}(\mathrm{dap})_{2} \mathrm{Cl}(1)$ & - & $\mathrm{O} / \mathrm{N}$ & $82 \%$ \\
\hline $2^{[c]}$ & $\mathrm{ABZ}(1.4)$ & $\mathrm{Cu}(\text { dap })_{2} \mathrm{Cl}(1)$ & - & $\mathrm{O} / \mathrm{N}$ & $78 \%$ \\
\hline 3 & $\mathrm{ABZ}(1.4)$ & $\mathrm{CuCl}_{2}(10)$ & - & $\mathrm{O} / \mathrm{N}$ & $62 \%$ \\
\hline 4 & TMS-N $\mathrm{N}_{3}(2)$ & - & PIDA (2) & $30 \mathrm{~min}$ & $56 \%$ \\
\hline 5 & TMS-N $\mathrm{N}_{3}(2)$ & $\mathrm{CuCl}_{2}(10)$ & PIDA (2) & $30 \mathrm{~min}$ & $94 \%$ \\
\hline 6 & TMS- $\mathrm{N}_{3}(1.5)$ & $\mathrm{CuCl}_{2}(10)$ & PIDA (1.5) & $30 \mathrm{~min}$ & $85 \%$ \\
\hline 7 & TMS-N ${ }_{3}(1.8)$ & $\mathrm{CuCl}_{2}(5)$ & PIDA (1.8) & $30 \mathrm{~min}$ & $\begin{array}{c}90 \% \\
\left(88 \%\left[{ }^{[d]}\right)\right.\end{array}$ \\
\hline
\end{tabular}

[a] Reaction conditions: $0.1 \mathrm{mmol}$ scale, $\mathrm{O} / \mathrm{N}$ : overnight. dap $=2,9-$ bis (paraanisyl) - 1,10 - phenanthroline. [b] Yields determined by ${ }^{1} \mathrm{H}$ NMR using $\mathrm{CH}_{2} \mathrm{Br}_{2}$ as internal standard. [c] Reaction performed under Green LED irradiation. [d] Isolated yield on $0.1 \mathrm{mmol}$ scale.

With optimized conditions in hands, we carried some preliminary scope investigations (Scheme 2). On a $0.3 \mathrm{mmol}$ scale, cyclopropene 1 delivered alkenyl nitrile 2 in 91\% yield. Dibenzyland ditrifluoroethyl-ester substituted cyclopropenes gave products 4 and 5 in $74 \%$ and $72 \%$ yield, respectively. Both electron-donating and -withdrawing groups in para-position on the aromatic substituent of the cyclopropene were compatible with the reaction (products 6 and 7). ${ }^{[2]}$ Meta-substitution was also tolerated (product $8,81 \%$ yield). The reaction proceeded as well with a 2-naphthyl substituent, delivering 9 in $94 \%$ yield. For $\mathbf{9}$, an $\mathrm{X}$-Ray structure could be obtained. ${ }^{[25]} \mathrm{An}$ ortho- $\mathrm{CF}_{3}$ substituted cyclopropene led to the formation of $\mathbf{1 0}$ in $\mathbf{7 7 \%}$ yield. As shown with examples 11, 12 and $\mathbf{1 3}$ for which only traces of the alkenyl nitriles were observed, having an aryl-substituent on the unsaturated bond was essential. With aryl groups both in position 1 and 2 of the cyclopropene ring, the reaction proceeded in high yield, with 14 isolated in $84 \%$ yield on a $1.6 \mathrm{mmol}$ scale, albeit with almost no stereoselectivity.

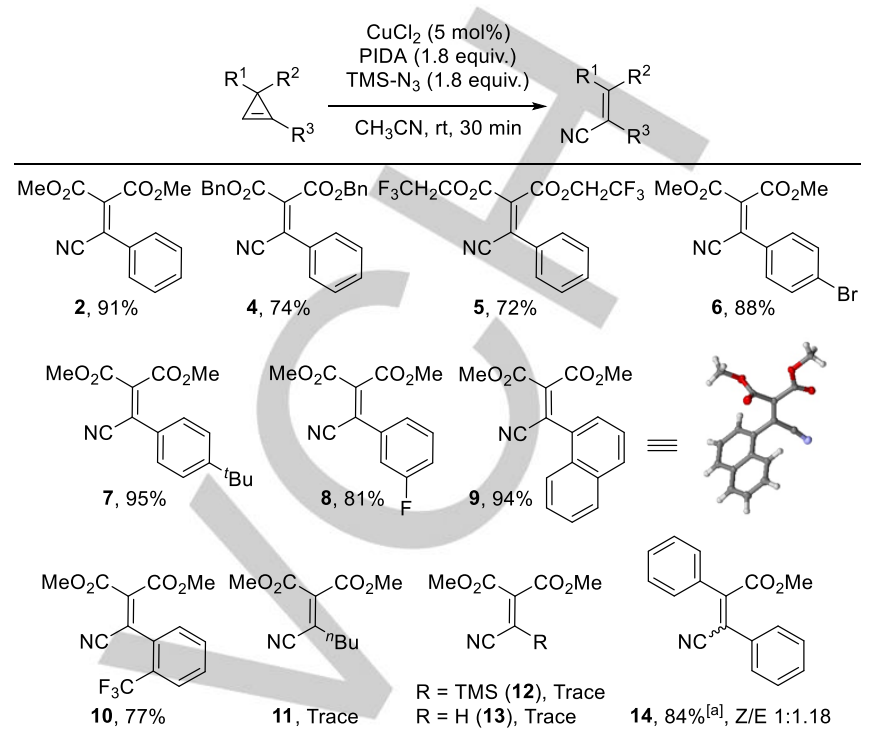

Scheme 2. Preliminary scope investigations of the radical amination. Reaction conditions: $0.3 \mathrm{mmol}$ scale. [a] Yield on $1.6 \mathrm{mmol}$ scale.

Alkenyl nitrile 2 could be obtained in $92 \%$ yield on gram scale (Scheme 3). Taking advantage of the electrophilic nature of 2 , it was successfully engaged in several transformations. A CoreyChaykovsky cyclopropanation led to 15 in 54\% yield. ${ }^{[26]}$ Alkene 2 underwent a $(3+2)$ annulation with allenoate 16, delivering cyclopentene 17 in $89 \%$ yield. ${ }^{[27]} 2$ could also be engaged in a Diels-Alder cycloaddition with Danishefsky's diene $18,{ }^{[28]}$ leading to cyclohexenone 19 after acidic deprotection in $64 \%$ yield. We then investigated the possibility to engage cyano-stilbene derivatives such as $\mathbf{1 4}$ into an oxidative photocyclization. ${ }^{[29]}$ Under irradiation, $E$ to $Z$ isomerization would occur, allowing converting both isomers to the product. While straightforward methods now exist for the synthesis of simple disubstituted stilbenes, ${ }^{[30]}$ accessing tetrasubstituted stilbenes with four different substituents as obtained in our work still constitutes an important challenge. ${ }^{[31]}$ In the presence of 1.1 equivalent of $\mathrm{DDQ}$ in acetonitrile $(0.05 \mathrm{M})$ under UV irradiation $(365 \mathrm{~nm})$ for 20 hours, cyano-phenanthrene 20 could be isolated in $74 \%$ yield.

A one-pot process from 1,2-diaryl substituted cyclopropenes allowed to directly access the corresponding PACs (Scheme 4, see SI for details). On a $0.3 \mathrm{mmol}$ scale, $\mathbf{2 0}$ could be isolated in $80 \%$ yield. A tert-butyl ester, a ketone and a trifluoromethyl group in position 1 of the cyclopropene led to phenanthrenes 21, 22 and 23 in $51-75 \%$ yields. Both electron-donating and -withdrawing substituents in para position of both aromatic groups were well tolerated, delivering products 24-27. Substitutions in ortho and meta positions were also tolerated (28-31). A 1-thienyl substituted cyclopropene gave heteroaromatic product 32 in $61 \%$ yield. 
a) Gram-scale Synthesis of Alkenyl Nitrile 2
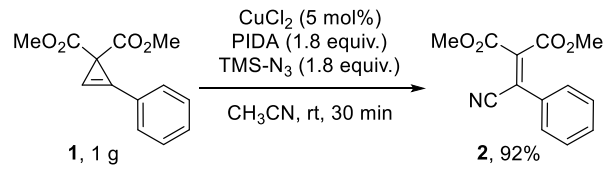

b) Further Transformations of Alkenyl Nitrile 2

$$
\begin{gathered}
\mathrm{SOMe}_{3} \mathrm{I}(1.4 \text { equiv.) } \\
\mathrm{DMSO}(1.2 \text { equiv.) } \\
\mathrm{DM} .1 \mathrm{M}), \mathrm{rt}, \mathrm{O} / \mathrm{N}
\end{gathered}
$$

$$
\mathrm{MeO}_{2} \mathrm{C} \overbrace{\mathrm{Ph}}^{\mathrm{CO}_{2} \mathrm{Me}}
$$$$
\mathrm{EtO}_{2} \mathrm{C} \text {. }
$$

$$
\text { i) }
$$

ii) TFA, DCM

c) Oxidative Photocyclization of 14

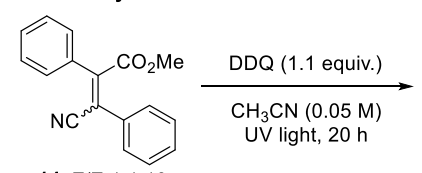

14, Z/E 1:1.18 most probably by hypervalent iodine reagent intermediates I or II, would give the nitrile product. ${ }^{[39]}$

The role of the copper catalyst is still unclear. When the reaction was performed with cyclopropene 39 in absence of $\mathrm{CuCl}_{2}$ (Scheme 5B), alkenyl nitrile $\mathbf{1 4}$ could be still isolated in $30 \%$ yield, but quinoline $\mathbf{4 0}$ was also obtained in $34 \%$. Quinoline $\mathbf{4 0}$ may be formed by addition of the iminyl radical in intermediate VIII onto the benzene ring, followed by oxidation and rearomatization. Its formation therefore further support path $\mathbf{b}$ of the proposed mechanism. One role for copper may be to recombine with the imine radical, preventing cyclization and facilitating formation of the nitrile via deprotonation, eventually after oxidation by the hypervalent iodine reagent. ${ }^{[40]}$
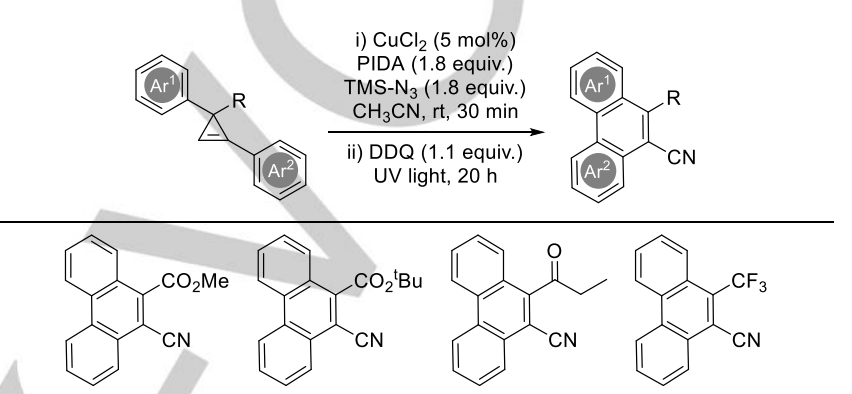
$20,80 \%$ 21, $75 \%$ 22, $51 \%$

Scheme 3. Gram-scale synthesis of 2 and further functionalizations of alkenyl nitriles 2 and 14. [a] Isolated as a 4.2:1 mixture of regioisomers, major regioisomer drawn.

We then investigated the synthesis of extended PACs. Cyclopropenes substituted with 2-naphthyl groups gave functionalized [4] helicenes 33-35 in good yields. Chrysene and benzo[g]chrysene derivatives could also be accessed (products $\mathbf{3 6}$ and $\mathbf{3 7}$ ). Substituted picene $\mathbf{3 8}$ was obtained in a slightly lower yield of $54 \%$. For all the substrates, full conversion of the starting material towards the corresponding alkenyl nitriles was observed. Hence when lower yields were obtained, the oxidative photocyclization was the limiting step.

Based on experimental observations and literature precedence in the use of azides to generate nitrile-containing compounds, ${ }^{\left[{ }^{32]}\right.}$ a speculative mechanism can be proposed (Scheme 5A). The reaction of PIDA with TMS- $\mathrm{N}_{3}$ would lead to the formation of unstable hypervalent iodine compounds I and II, which will dissociate into a iodanyl and an azide radical III and IV. ${ }^{[22,33] \text { IV }}$ would most likely add onto the C-C double bond of the cyclopropene to generate azido-cyclopropyl radical V. ${ }^{[34]}$ Two different paths can then be proposed. In path A, cyclopropyl radical $\mathbf{V}$ would be oxidized to cyclopropyl cation $\mathbf{V I}$, probably by an hypervalent iodine intermediate. From the latter a ring-opening rearrangement initiated by the azide group would occur, leading to the formation of VII. From VII, a base-mediated (azide or acetate $)^{[35]}$ fragmentation would then lead to the alkenyl nitrile upon loss of nitrogen. ${ }^{[36]}$ Another possibility from intermediate $\mathbf{V}$ would be a direct rearrangement triggered by the azide group with loss of nitrogen to give iminyl radical VIII (path b). ${ }^{[7]}$ The rearrangement of cyclopropyl to allyl radicals is a relatively easy process, with an energy barrier of only $22 \mathrm{kcal} / \mathrm{mol}$ for the cyclopropyl radical. ${ }^{[38]}$ In this case, coupling it with nitrogen release may even lower further the activation energy, although this remains highly speculative. From this intermediate, oxidation, 23, $65 \%$

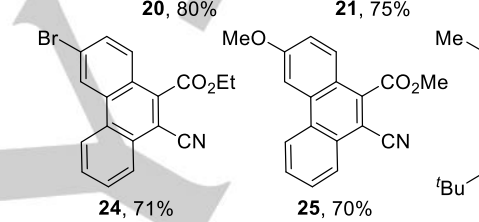<smiles>COC(=O)c1c(C)ccc2c(C(C)=O)c(C#N)c3ccc(C)cc3c12</smiles><smiles>COC(=O)c1c(C#N)c2ccccc2c2c(C#N)c(C(C)=O)c3c(Cl)cccc3c12</smiles><smiles>CC(=O)c1c(C#N)c2ccc(C)cc2c2cc(F)cc(Cl)c12</smiles><smiles>CC(=O)c1c(C#N)c2cc(C(F)(F)F)cc(C(F)(F)F)c2c2ccccc12</smiles><smiles>CCCCCc1cc2c(C(=O)OC)c(C#N)c3ccccc3c2s1</smiles>
$29,70 \%$

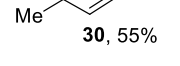
$\mathrm{CF}_{3} 31,68 \%$<smiles>CCOc1ccc2c(C#N)c(C(C)=O)c3ccc4ccccc4c3c2c1</smiles>
33, $76 \%$<smiles>COc1ccc2c(C(C)=O)c(C#N)c3ccc4ccccc4c3c2c1</smiles><smiles>CCOC(=O)c1c(C#N)c2ccc(Br)cc2c2c1ccc1ccccc12</smiles>

32, $61 \%$

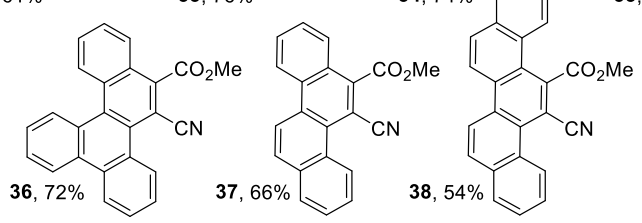

Scheme 4. Scope of the one-pot radical amination / oxidative photocyclization Reaction conditions: $0.3 \mathrm{mmol}$ scale.

In conclusion, we have developed a radical amination/ringopening of cyclopropenes using azides as nitrogen source. This transformation constitutes the first example of addition of an $\mathrm{N}$ centered radical onto cyclopropenes and is one of the few reports of a ring-opening event after a radical functionalization of the unsaturated bond. The obtained tetrasubstituted alkenyl nitriles could be further engaged in several transformations. In the case of 1,2-diaryl-substituted cyclopropenes, a one-pot process could be developed to access highly functionalized PACs. This methodology therefore provides a new way to use cyclopropenes as starting materials for the synthesis of valuable nitrile-containing scaffolds using commercially available reagents. 


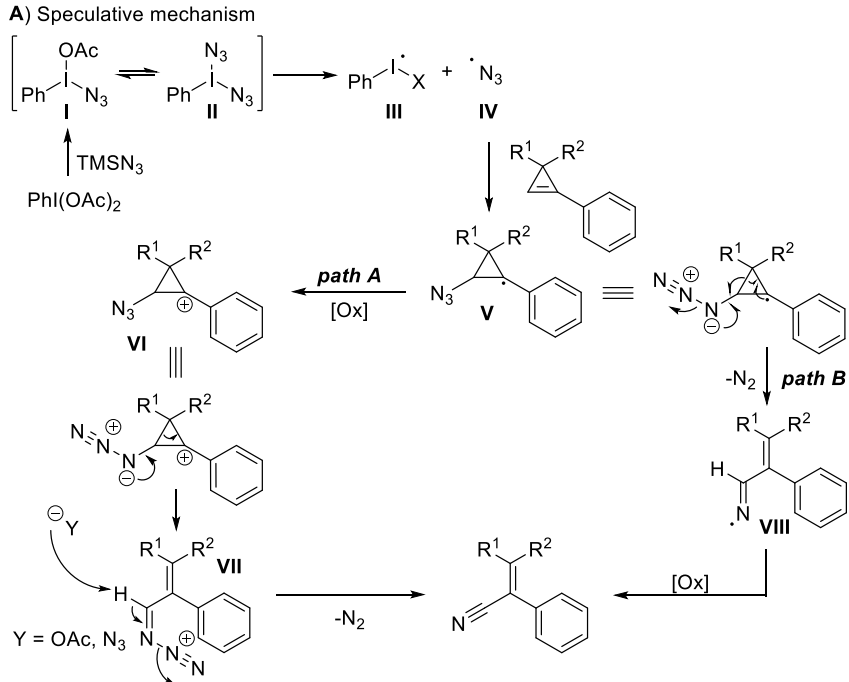

B) Control experiment in absence of $\mathrm{CuCl}_{2}$

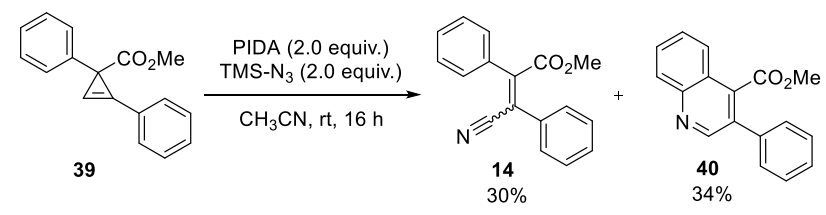

Scheme 5. Speculative mechanism and control experiment in absence of copper catalyst with cyclopropene 39 .

\section{Acknowledgements}

We thank the Swiss National Science Foundation (SNSF, grant no. 200020-182798) and EPFL for financial support. We thank Dr R. Scopelliti and Dr F. F. Tirani from ISIC at EPFL for X-ray analysis.

Keywords: cyclopropenes • amination $•$ radicals $•$ nitriles $•$ polycyclic aromatic compounds

[1] a) Z. Rappoport, The Chemistry of the Cyano Group, Wiley, London, 1970; b) J. S. Miller, J. L. Manson, Acc. Chem. Res. 2001, 34, 563; c) F. F. Fleming, L. Yao, P. C. Ravikumar, L. Funk, B. C. Shook, J. Med. Chem. 2010, 53, 7902; d) Y. Shen, C. F. Chen, Chem. Rev. 2012, 112, 1463; e) B. Tóth, J. Hohmann, A. Vasas, J. Nat. Prod. 2018, 81, 661; f) G. Albano, G. Pescitelli, L. Di Bari, Chem. Rev. 2020, 120, 10145.

[2] a) G. V. Sawle, D. J. Burn, P. K. Morrish, A. A. Lammertsma, B. J. Snow, S. Luthra, S. Osman, D. J. Brooks, Neurology 1994, 44, 1292; b) A. Schrag, Lancet Neurol. 2005, 4, 366.

[3] a) J. M. Molina, P. Cahn, B. Grinsztejn, A. Lazzarin, A. Mills, M. Saag, K. Supparatpinyo, S. Walmsley, H. Crauwels, L. T. Rimsky, S. Vanveggel, K. Boven, Lancet 2011, 378, 238; b) M. Sharma, L. D. Saravolatz, J. Antimicrob. Chemother. 2013, 68, 250.

[4] F. F. Fleming, Q. Wang, Chem. Rev. 2003, 103, 2035.

[5] a) A. Kovács, A. Vasas, J. Hohmann, Phytochemistry 2008, 69, 1084; b) J. C. Lin, S. C. Yang, T. M. Hong, S. L. Yu, S. Qian, W. Linyi, H. Y. Chen, P. C. Yang, K. H. Lee, J. Med. Chem. 2009, 52, 1903; c) X. Yang, Q. Shi, K. F. Bastow, K. H. Lee, Org. Lett. 2010, 12, 1416; d) J. Han, Z. Xian, Y. Zhang, J. Liu, A. Liang, Front. Pharmacol. 2019, 10, 648.

[6] a) C. Wang, H. Dong, W. Hu, Y. Liu, D. Zhu, Chem. Rev. 2012, 112, 2208; b) Z. He, X. Xu, X. Zheng, T. Ming, Q. Miao, Chem. Sci. 2013, 4, 4525; c) M. Li, C. An, T. Marszalek, X. Guo, Y. Z. Long, H. Yin, C. Gu, M. Baumgarten, W. Pisula, K. Müllen, Chem. Mater. 2015, 27, 2218.
[7] a) S. Wang, X. Yan, Z. Cheng, H. Zhang, Y. Liu, Y. Wang, Angew. Chem Int. Ed. 2015, 54, 13068; b) S. Jhulki, A. K. Mishra, T. J. Chow, J. N Moorthy, Chem. Eur. J. 2016, 22, 9375; c) S. Jhulki, A. K. Mishra, A. Ghosh, T. J. Chow, J. N. Moorthy, J. Mater. Chem. C 2016, 4, 9310; d) S. Jhulki, A. K. Mishra, T. J. Chow, J. N. Moorthy, New J. Chem. 2017, 41, 14730; e) S. Kang, H. Lee, H. Jung, M. Jo, M. Jung, J. Park, Dye Pigment. 2018, 156, 299.

[8] Review: a) A. J. Floyd, S. F. Dyke, S. E. Ward, Chem. Rev. 1976, 76 509; Recent examples: b) D. Peña, D. Pérez, E. Guitián, L. Castedo, J Am. Chem. Soc. 1999, 121, 5827; c) E. Yoshikawa, Y. Yamamoto Angew. Chem., Int. Ed. 2000, 39, 173; d) V. Mamane, P. Hannen, A. Fürstner, Chem. Eur. J. 2004, 10, 4556; e) D. C. Harrowven, I. L. Guy, L. Nanson, Angew. Chem., Int. Ed. 2006, 45, 2242; f) Y. B. Zhao, B. Mariampillai, D. A. Candito, B. Laleu, M. Z. Li, M. Lautens, Angew. Chem., Int. Ed. 2009, 48, 1849; g) A. Matsumoto, L. llies, E. Nakamura, J. Am. Chem. Soc. 2011, 133, 6557; h) C. C. McAtee, P. S. Riehl, C. S. Schindler, J. Am. Chem. Soc. 2017, 139, 2960; i) Z. Zhao, L. H. Britt, G. K. Murphy, Chem. Eur. J. 2018, 24, 17002.

[9] For recent reviews, see: a) R. Vicente, Synthesis 2016, 48, 2343; b) P. Li, X. Zhang, M. Shi, Chem. Commun. 2020, 56, 5457; c) B. Prasad Raiguru, S. Nayak, D. Ranjan Mishra, T. Das, S. Mohapatra, N. Priyadarsini Mishra, Asian J. Org. Chem. 2020, 9, 1088.

[10] For recent reviews, see: a) I. Marek, S. Simaan, A. Masarwa, Angew. Chem., Int. Ed. 2007, 46, 7364; b) L. Dian, I. Marek, Chem. Rev. 2018 118, 8415. For selected recent examples: c) Y. Luo, H.-L. Teng, M Nishiura, Z. Hou, Angew. Chem., Int. Ed. 2017, 56, 9207; d) L. Dian, I. Marek, Angew. Chem., Int. Ed. 2018, 57, 3682; e) H. Zhang, W. Huang, T. Wang, F. Meng, Angew. Chem., Int. Ed. 2019, 58, 11049.

[11] For selected reviews, see: a) F. Miege, C. Meyer, J. Cossy, Beilstein J. Org. Chem. 2011, 7, 717; b) R. Vicente, Chem. Rev. 2020, DOI 10.1021/acs.chemrev.0c00151. For selected examples, see: c) F. Miege, C. Meyer, J. Cossy, Angew. Chem., Int. Ed. 2011, 50, 5932; d) A. Archambeau, F. Miege, C. Meyer, J. Cossy, Angew. Chem., Int. Ed. 2012, 51, 11540; e) S. Mata, L. A. López, R. Vicente, Angew. Chem., Int. Ed. 2017, 56, 7930. Xiao and co-workers recently reported a different approach based on palladium catalysis: Z. Jiang, S.-L. Niu, Q. Zeng, Q. Ouyang, Y.-C. Chen, Q. Xiao, Angew. Chem., Int. Ed. 2020, DOI: doi:10.1002/anie.202008886.

[12] S. Yamago, S. Ejiri, E. Nakamura, Chem. Lett. 1994, 23, 1889.

[13] a) N. Legrand, B. Quiclet-Sire, S. Z. Zard, Tetrahedron Lett. 2000, 41, 9815 ; b) Z. Ferjančić, Ž. Čeković, R. N. Saičić, Tetrahedron Lett. 2000 , $41,2979$.

[14] a) M. Ueda, N. Doi, H. Miyagawa, S. Sugita, N. Takeda, T. Shinada, O. Miyata, Chem. Commun. 2015, 51, 4204; b) N. Doi, N. Takeda, O. Miyata, M. Ueda, J. Org. Chem. 2016, 81, 7855.

[15] N. S. Dange, F. Robert, Y. Landais, Org. Lett. 2016, 18, 6156.

[16] B. Muriel, A. Gagnebin, J. Waser, Chem. Sci. 2019, 10, 10716.

[17] N. S. Dange, A. Hussain Jatoi, F. Robert, Y. Landais, Org. Lett. 2017, 19, 3652.

[18] a) A. Parra, L. Amenós, M. Guisán-Ceinos, A. López, J. L. García Ruano, M. Tortosa, J. Am. Chem. Soc. 2014, 136, 15833; b) M. Simaan, I. Marek, Angew. Chem., Int. Ed. 2018, 57, 1543; c) Z. Li, M. Zhang, Y. Zhang, S Liu, J. Zhao, Q. Zhang, Org. Lett. 2019, 21, 5432; d) S. Feng, H. Hao, P. Liu, S. L. Buchwald, ACS Catal. 2020, 10, 282.

[19] a) T. K. Hyster, T. Rovis, Synlett 2013, 24, 1842; b) H. L. Teng, Y. Luo, B. Wang, L. Zhang, M. Nishiura, Z. Hou, Angew. Chem., Int. Ed. 2016 55, 15406; c) N. Semakul, K. E. Jackson, R. S. Paton, T. Rovis, Chem. Sci. 2017, 8, 1015; d) Z. Li, J. Zhao, B. Sun, T. Zhou, M. Liu, S. Liu, M. Zhang, Q. Zhang, J. Am. Chem. Soc. 2017, 139, 11702; e) H. L. Teng, Y. Luo, M. Nishiura, Z. Hou, J. Am. Chem. Soc. 2017, 139, 16506.

[20] a) C. Qin, N. Jiao, J. Am. Chem. Soc. 2010, 132, 15893; b) W. Zhou, J. Xu, L. Zhang, N. Jiao, Org. Lett. 2010, 12, 2888; c) W. Zhou, J. Xu, L. Zhang, N. Jiao, Synlett 2011, 2011, 887; d) X. Huang, N. Jiao, Org. Biomol. Chem. 2014, 12, 4324; e) X. Huang, X. Li, N. Jiao, Chem. Sci. 2015, 6, 6355.

[21] S. Alazet, J. Preindl, R. Simonet-Davin, S. Nicolai, A. Nanchen, T. Meyer, J. Waser, J. Org. Chem. 2018, 83, 12334.

[22] a) E. Zbiral, G. Nestler, Tetrahedron 1970, 26, 2945; b) P. Magnus, J. Lacour, J. Am. Chem. Soc. 1992, 114, 767; c) C. M. Pedersen, L. G. 
Marinescu, M. Bols, Org. Biomol. Chem. 2005, 3, 816; d) X. F. Xia, Z. Gu, W. Liu, H. Wang, Y. Xia, H. Gao, X. Liu, Y. M. Liang, J. Org. Chem. 2015, 80, 290; e) Z. Wu, R. Ren, C. Zhu, Angew. Chem., Int. Ed. 2016 $55,10821$.

[23] H. Ma, D. Li, W. Yu, Org. Lett. 2016, 18, 868.

[24] Decomposition was observed with a more electron-rich substrate bearing a para-methoxy group.

[25] The structure of $\mathbf{9}$ was confirmed by $\mathrm{X}$-ray analysis. Deposition Number 2027422 (for 9) contain the supplementary crystallo-graphic data for this paper. These data are provided free of charge by the joint Cambridge Crystallographic Data Centre and Fachinformationszentrum Karlsruhe Access Structures service www.ccdc.cam.ac.uk/structures.

[26] C. A. Carson, M. A. Kerr, Org. Lett. 2009, 11, 777.

[27] R. Liu, Z. Qin, B. Fan, R. Li, R. Zhou, Z. He, J. Org. Chem. 2019, 84 12490.

[28] J. M. Eagan, M. Hori, J. Wu, K. S. Kanyiva, S. A. Snyder, Angew. Chem., Int. Ed. 2015, 54, 7842.

[29] a) F. B. Mallory, C. W. Mallory, Org. React. 2005, 30, 1-456; b) A. Del Tito, H. O. Abdulla, D. Ravelli, S. Protti, M. Fagnoni, Beilstein J. Org. Chem. 2020, 16, 1476.

[30] a) K. B. Becker, Synthesis 1983, 1983, 341; b) Z. A. Khan, A. Iqbal, S. A. Shahzad, Mol. Diversity 2017, 21, 483.

[31] To the best of our knowledge there is only one report of the synthesis of a stilbene derivative bearing an ester and a cyano group, proceeding via the iodocyanation of an alkyne: N. Sakata, K. Sasakura, G. Matsushita, K. Okamoto, K. Ohe, Org. Lett. 2017, 19, 3422.

[32] T. Wang, N. Jiao, Acc. Chem. Res. 2014, 47, 1137.

[33] a) F. Fontana, F. Minisci, M. Y. Yong, Z. Lihua, Tetrahedron Lett. 1993 34, 2517; b) Y. Kita, H. Tohma, T. Takada, S. Mitoh, S. Fujita, M. Gyoten Synlett 1994, 1994, 427.

[34] Attempts to trap radical $\mathbf{V}$ via $\mathrm{H}$-transfer from solvents such as THF or dioxane or halogen transfer from $\mathrm{CCl}_{3} \mathrm{Br}$ were not successful.

[35] Acetate is the strongest base in less polar solvents, see: F. G. Bordwell, Acc. Chem. Res. 1988, 21, 456.

[36] W. Zhou, L. Zhang, N. Jiao, Angew. Chem., Int. Ed. 2009, 48, 7094.

[37] a) P. C. Montevecchi, M. L. Navacchia, P. Spagnolo, J. Org. Chem. 1997, 62, 5846; b) X. Sun, X. Li, S. Song, Y. Zhu, Y. F. Liang, N. Jiao, J. Am. Chem. Soc. 2015, 137, 6059.

[38] a) R. Walsh, Int. J. Chem. Kinet. 1970, 2, 71; b) D. J. Mann, W. L. Hase, J. Am. Chem. Soc. 2002, 124, 3208.

[39] G. Bencivenni, T. Lanza, R. Leardini, M. Minozzi, D. Nanni, P. Spagnolo, G. Zanardi, J. Org. Chem. 2008, 73, 4721.

[40] We thank a reviewer for suggesting us this pathway for the conversion of the imine radical to the nitrile product. 


\section{Entry for the Table of Contents}

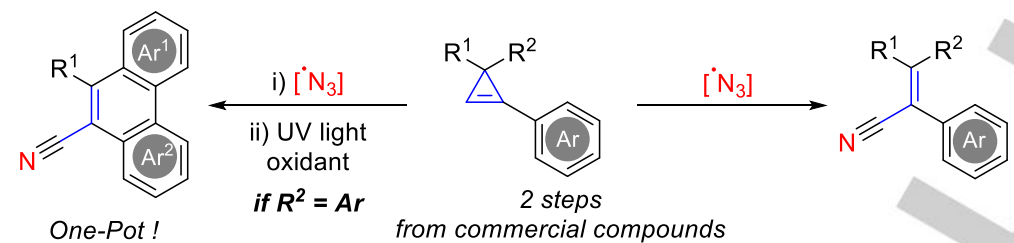

A radical amination of cyclopropenes has been developed. The transformation proceeds through a cleavage of the three-membered ring and provides access to alkenyl nitriles, without the need of an external cyanide source. With 1,2-diaryl substituted cyclopropenes, a one-pot process could be developed to access a wide range of highly functionalized polycyclic aromatic compounds.

Institute and/or researcher Twitter usernames: @LcsoLab, @EPFL_CHEM_Tweet. 


\section{Table of Contents}

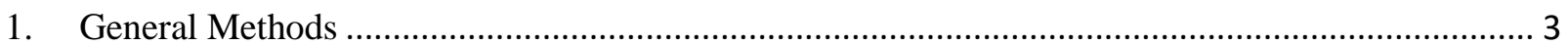

2. Preparation of Starting Materials...................................................................................... 4

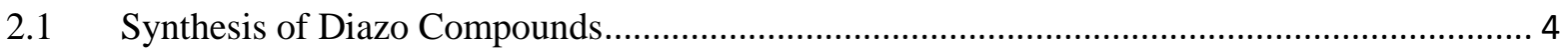

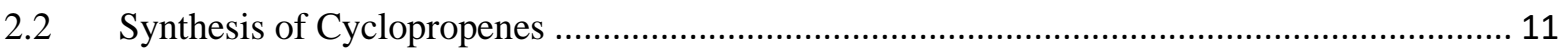

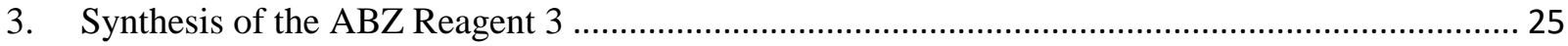

4. Radical Amination of Cyclopropenes for the Synthesis of Alkenyl Nitriles................................ 27

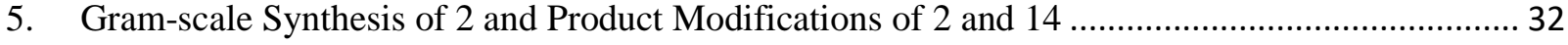

6. Optimization of the One-pot Synthesis of PACs from Cyclopropenes ....................................... 35

7. One-pot Synthesis of PACs from Cyclopropenes …................................................................... 36

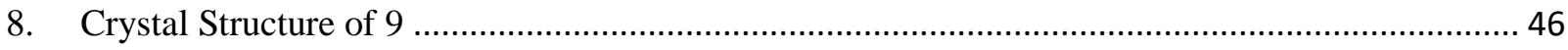

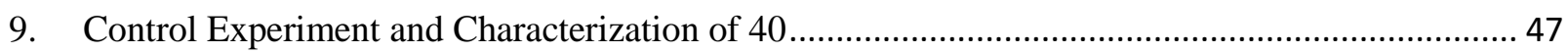

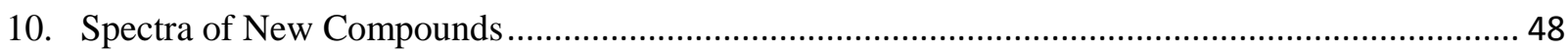




\section{General Methods}

All reactions were carried out in oven dried glassware under an atmosphere of nitrogen, unless stated otherwise. For quantitative flash chromatography technical grade solvents were used. For flash chromatography for analysis, HPLC grade solvents from Sigma-Aldrich were used. THF, $\mathrm{Et}_{2} \mathrm{O}, \mathrm{CH}_{3} \mathrm{CN}$, toluene, hexane and $\mathrm{CH}_{2} \mathrm{Cl}_{2}$ were dried by passage over activated alumina under nitrogen atmosphere $\left(\mathrm{H}_{2} \mathrm{O}\right.$ content $<10 \mathrm{ppm}$, Karl-Fischer titration). The solvents were degassed by Freeze-Pump-Thaw method when mentioned. All chemicals were purchased from Acros, Aldrich, Fluka, VWR, Aplichem, Fluorochem or Merck and used as such unless stated otherwise. Chromatographic purification was performed as flash chromatography using Macherey-Nagel silica 40-63, $60 \AA$, using the solvents indicated as eluent with 0.1-0.5 bar pressure. TLC was performed on Merck silica gel 60 F254 TLC glass plates or aluminium plates and visualized with UV light, permanganate stain, CAN stain or Anisaldehyde stain. Melting points were measured on a Büchi B-540 melting point apparatus using open glass capillaries, the data is uncorrected. ${ }^{1} \mathrm{H}$ NMR spectra were recorded on a Brucker DPX-400 $400 \mathrm{MHz}$ spectrometer in chloroform- $d$, all signals are reported in ppm with the internal chloroform signal at $7.26 \mathrm{ppm}$. The data is being reported as $(\mathrm{s}=$ singlet, $\mathrm{d}=$ doublet, $\mathrm{t}=$ triplet, $\mathrm{q}=$ quadruplet, $\mathrm{qi}=$ quintet, $\mathrm{m}=$ multiplet or unresolved, br = broad signal, app = apparent, coupling constant(s) in Hz, integration, interpretation). ${ }^{19} \mathrm{~F}-\mathrm{NMR}$ spectra were recorded on a Bruker DPX-400 $376 \mathrm{MHz}$ spectrometer in $\mathrm{CDCl}_{3}$ or $\mathrm{SO}\left(\mathrm{CD}_{3}\right)_{2} \cdot{ }^{13} \mathrm{C}$-NMR spectra were recorded with ${ }^{1} \mathrm{H}$-decoupling on a Brucker DPX-400 $100 \mathrm{MHz}$ spectrometer in chloroform-d, all signals are reported in ppm with the internal chloroform signal at $77.0 \mathrm{ppm}$. Infrared spectra were recorded on a JASCO FT-IR B4100 spectrophotometer with an ATR PRO410-S and a ZnSe prisma and are reported as $\mathrm{cm}^{-1}(\mathrm{w}=$ weak, $\mathrm{m}=$ medium, $\mathrm{s}=$ strong, br = broad). High resolution mass spectrometric measurements were performed by the mass spectrometry service of ISIC at the EPFL on a MICROMASS (ESI) Q-TOF Ultima API.

For reactions under the irradiation of UV light $(365 \mathrm{~nm})$, reactions were performed in borosilicate glass tubes which were placed around $7 \mathrm{~cm}$ far away from lamps (CAMAG UV Lamp 4, longwave UV light $365 \mathrm{~nm}$ ) in a Rayonet RPR-100 photochemical reactor. For reactions under the irradiation of green light, reactions were performed in $12 * 75 \mathrm{~mm}$ borosilicate glass tubes which were hold using a rack for test tubes placed at the center of a crystallization flask. On this flask were attached the green LEDs (LED ribbon cable with open ends, Barthelme Y51515213 182007 $12 \mathrm{~V} 502 \mathrm{~cm}$ green $1 \mathrm{pc}(\mathrm{s})$, purchased directly on www.conrad.ch/fr). The distance between the LEDs and the test tubes was approximately 3-4 cm. Long irradiation for more than $2 \mathrm{~h}$ resulted in the temperature increasing up to $34^{\circ} \mathrm{C}$. 


\section{Preparation of Starting Materials}

\subsection{Synthesis of Diazo Compounds}

General procedure A: Synthesis of $\alpha$-aryldiazo compounds.<smiles>[R]Cc1cc[R1]cc1</smiles>

Following a slightly modified procedure, ${ }^{1} \mathrm{DBU}$ (1.6 equiv.) and the indicated $\alpha$-aryl-acetate or ketone (1.0 equiv.) were added to a solution of $p \mathrm{ABSA}$ (1.5 equiv.) in $\mathrm{CH}_{3} \mathrm{CN}(0.5 \mathrm{M})$ at room temperature and the resulting mixture was stirred for 18 hours. The reaction mixture was then diluted with distilled water and extracted with diethyl ether. The combined organic layers were washed with a $10 \% \mathrm{NaHCO}_{3}$ solution and brine, then dried over $\mathrm{MgSO}_{4}$, filtered, and concentrated under reduced pressure. The crude residue was purified by column chromatography with the indicated solvents.

Dimethyl 2-diazomalonate (42)

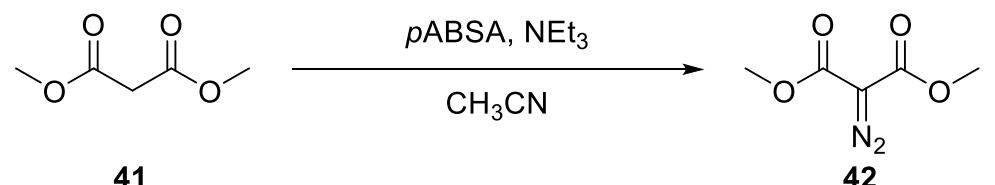

Following a modified procedure, ${ }^{2}$ triethylamine $(13.4 \mathrm{~mL}, 96.0 \mathrm{mmol}, 2.4$ equiv.) and dimethyl malonate (41) $(4.60 \mathrm{~mL}, 40.0 \mathrm{mmol}, 1.0$ equiv.) were added to a solution of $p$ ABSA (14.4 g, 60.0 mmol, 1.5 equiv.) in $\mathrm{CH}_{3} \mathrm{CN}(160 \mathrm{~mL})$ at room temperature and the resulting mixture was stirred for 18 hours at room temperature. Thereafter the mixture was filtered and the solvent was evaporated. The residue was triturated with $\mathrm{CH}_{2} \mathrm{Cl}_{2}(100 \mathrm{~mL})$, the remaining solids were filtered off and the solvent was evaporated. The crude residue was purified by column chromatography ( $\mathrm{SiO}_{2}$, Pentane:EtOAc 95:5 to 90:10) to afford compound 42 (6.26 g, $39.6 \mathrm{mmol}, 99 \%$ yield) as a yellow oil. ${ }^{1} \mathbf{H}$ NMR (400 MHz, Chloroform- $\left.d\right) \delta 3.84\left(\mathrm{~s}, 6 \mathrm{H}, \mathrm{CH}_{3}\right) ;{ }^{13} \mathbf{C}$ NMR (101 MHz, Chloroform- $\boldsymbol{d}) \delta$ 161.2, 52.4. One carbon was not resolved. The characterization data is corresponding to the reported values. ${ }^{2}$

Dibenzyl 2-diazomalonate (44)<smiles>CCN(CC)C(=O)C(=O)OCc1ccccc1</smiles>

${ }^{1}$ W.-Y. Yu, Y.-T. Tsoi, Z. Zhou, A. S. C. Chan, Org. Lett. 2009, 11, 469-472.

${ }^{2}$ F. de Nanteuil, J. Waser, Angew. Chem. Int. Ed. 2011, 50, 12075-12079. 
Following a modified procedure, ${ }^{3}$ triethylamine $(1.0 \mathrm{~mL}, 7.2 \mathrm{mmol}, 2.4$ equiv.) and dibenzyl malonate (43) $(0.75 \mathrm{~mL}, 4.0 \mathrm{mmol}, 1.0$ equiv.) were added to a solution of pABSA (1.08 $\mathrm{g}, 4.50$ mmol, 1.5 equiv.) in $\mathrm{CH}_{3} \mathrm{CN}$ (16.0 mL) at room temperature and the resulting mixture was stirred for 18 hours at room temperature. Thereafter the mixture was filtered and the solvent was evaporated. The residue was triturated with $\mathrm{CH}_{2} \mathrm{Cl}_{2}(15 \mathrm{~mL})$, the remaining solids were filtered off and the solvent was evaporated. The crude residue was purified by column chromatography $\left(\mathrm{SiO}_{2}\right.$, Pentane:EtOAc 95:5) to afford compound 44 (889 mg, $2.86 \mathrm{mmol}, 95 \%$ yield) as a yellow oil. ${ }^{\mathbf{1}} \mathbf{H}$ NMR (400 MHz, Chloroform-d) $\delta$ 7.39- $7.34(\mathrm{~m}, 10 \mathrm{H}, \mathrm{ArH}), 5.28\left(\mathrm{~s}, 4 \mathrm{H}, \mathrm{CH}_{2}\right) ;{ }^{\mathbf{1 3}} \mathbf{C}$ NMR (101 MHz, Chloroform- $\boldsymbol{d}$ ) $\delta 160.9,135.3,128.8,128.6,128.5,67.3$. One carbon was not resolved. The characterization data correspond to the reported values. ${ }^{3}$

Bis(2,2,2-trifluoroethyl) malonate (47)

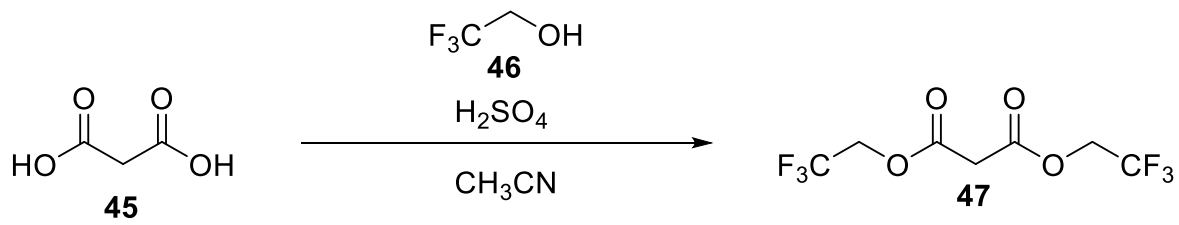

Following a reported procedure, ${ }^{2} \mathrm{H}_{2} \mathrm{SO}_{4}(1.00 \mathrm{~mL}, 18.8 \mathrm{mmol}, 0.25$ equiv.) was added to a solution of trifluoroethanol (46) (29.9 mL, $415 \mathrm{mmol}, 5.4$ equiv.) and malonic acid 45 (8.00 g, $77.0 \mathrm{mmol}$, 1.0 equiv.) in toluene $(40.0 \mathrm{~mL})$ and the resulting mixture was heated to reflux for 8 hours. After cooling to room temperature, toluene $(80.0 \mathrm{~mL})$ was added and the mixture was washed with aq. $\mathrm{NaOH}(200 \mathrm{~mL}, 1 \mathrm{M})$, water $(200 \mathrm{~mL})$ and brine $(200 \mathrm{~mL})$. The organic layer was dried over $\mathrm{MgSO}_{4}$ and the solvent was evaporated under reduced pressure to afford the title compound 47 (6.80 g, 25.4 mmol, 33\% yield) as a colorless oil. ${ }^{1} \mathbf{H}$ NMR (400 MHz, Chloroform-d) $\delta 4.55$ (q, $\left.J=8.2 \mathrm{~Hz}, 4 \mathrm{H}, \mathrm{OCH}_{2}\right), 3.61\left(\mathrm{~s}, 2 \mathrm{H}, \mathrm{CH}_{2}\right) ;{ }^{\mathbf{1 3}} \mathbf{C}$ NMR $(\mathbf{1 0 1} \mathbf{M H z}$, Chloroform-d) $\delta 164.4,122.8(\mathrm{q}, J$ $=277.3 \mathrm{~Hz}), 61.4(\mathrm{q}, J=37.0 \mathrm{~Hz}), 40.3$. The characterization data correspond to the reported values. $^{2}$

Bis(2,2,2-trifluoroethyl) 2-diazomalonate (48)

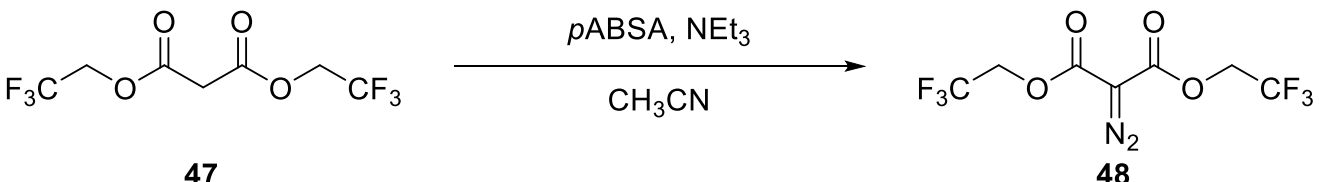

Following a modified procedure, ${ }^{2}$ triethylamine $(6.00 \mathrm{~mL}, 43.3 \mathrm{mmol}, 2.4$ equiv. $)$ and bis(trifluorethyl)malonate (47) (4.84 g, $18.0 \mathrm{mmol}, 1.0$ equiv.) were added to a solution of $p$ ABSA (6.50 g, $27.1 \mathrm{mmol}, 1.5$ equiv.) in $\mathrm{CH}_{3} \mathrm{CN}(72.0 \mathrm{~mL})$ at room temperature and the resulting mixture was stirred for 18 hours. Thereafter the mixture was filtered and the solvent was evaporated. The residue was triturated with $\mathrm{CH}_{2} \mathrm{Cl}_{2}(50 \mathrm{~mL})$, the solids were filtered off and the solvent was evaporated. The crude residue was purified by column chromatography $\left(\mathrm{SiO}_{2}\right.$, Pentane:EtOAc 95:5 to $90: 10)$ to afford compound 48 (5.26 g, $17.9 \mathrm{mmol}, 99 \%$ yield) as a yellow oil. ${ }^{\mathbf{1}} \mathbf{H}$ NMR (400 MHz, Chloroform-d) $\delta 4.62$ (q, $\left.4 \mathrm{H}, J=8.2 \mathrm{~Hz}, \mathrm{CH}_{2}\right) ;{ }^{13} \mathrm{C}$ NMR (101 MHz, Chloroform-d) $\delta$

${ }^{3}$ F. de Nanteuil, J. Loup, J. Waser, Org. Lett. 2013, 15, 3738-3741. 
158.7, $122.6(\mathrm{q}, J=277.3 \mathrm{~Hz}), 60.9$ (q, $J=37.0 \mathrm{~Hz})$. One carbon was not resolved. The characterization data correspond to the reported values. ${ }^{2}$

\section{Methyl 2-diazo-2-phenylacetate (49)}

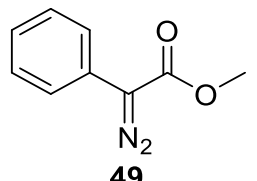

Following the general procedure A, starting from methyl 2-phenylacetate $(3.00 \mathrm{~mL}, 21.3 \mathrm{mmol}$, 1.0 equiv.), the title compound 49 was obtained after purification by column chromatography ( $\mathrm{SiO}_{2}$, Pentane:EtOAc 95:5) as a red oil (2.72 g, $15.4 \mathrm{mmol}, 73 \%$ yield). ${ }^{\mathbf{1}} \mathbf{H}$ NMR (400 MHz, Chloroform-d) $\delta 7.53-7.45(\mathrm{~m}, 2 \mathrm{H}, \mathrm{ArH}), 7.43-7.33(\mathrm{~m}, 2 \mathrm{H}, \operatorname{Ar} H), 7.23-7.14(\mathrm{~m}, 1 \mathrm{H}, \operatorname{Ar} H)$, 3.87 (s, 3H, $\left.\mathrm{CO}_{2} \mathrm{CH}_{3}\right) ;{ }^{13} \mathbf{C}$ NMR (101 MHz, Chloroform-d) $\delta 168.1,131.5,128.3,127.7,126.4$, 54.3. One carbon was not resolved. The characterization data correspond to the reported values. ${ }^{4}$

\section{Tert-butyl 2-diazo-2-phenylacetate (52)}

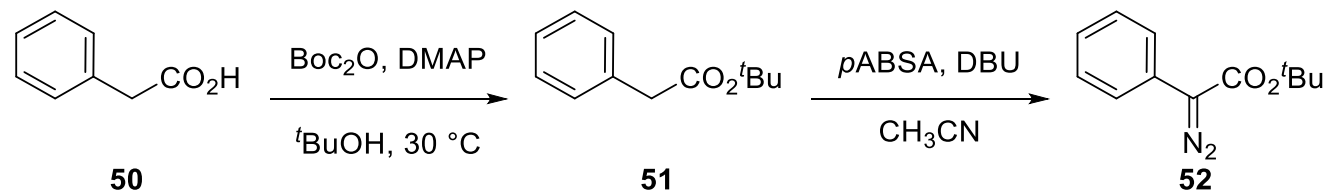

Following a reported procedure, ${ }^{5}$ 2-phenylacetic acid (50) (1.09 g, 8.00 mmol, 1.0 equiv), $\mathrm{Boc}_{2} \mathrm{O}$ (3.90 mL, $16.8 \mathrm{mmol}, 2.1$ equiv.) and DMAP (293 mg, $2.40 \mathrm{mmol}, 0.3$ equiv.) were dissolved in ${ }^{t} \mathrm{BuOH}(20 \mathrm{~mL})$. The mixture was stirred at $30{ }^{\circ} \mathrm{C}$ overnight. After cooling to room temperature, the solvent was evaporated and the crude residue was purified by column chromatography on Biotage (Büchi flashpure cartridge $40 \mathrm{~g}$, Pentane:EtOAc 98:2) to afford compound 51 (673 mg, $3.50 \mathrm{mmol}, 44 \%$ yield) as a colorless oil. ${ }^{1} \mathbf{H}$ NMR (400 MHz, Chloroform-d) $\delta$ 7.37-7.04 (m, 5H, $\mathrm{ArH}), 3.45(\mathrm{~s}, 2 \mathrm{H}, \mathrm{CH}), 1.36\left(\mathrm{~s}, 9 \mathrm{H},{ }^{t} \mathrm{Bu}\right) ;{ }^{13} \mathrm{C}$ NMR (101 MHz, Chloroform-d) $\delta 171.2$, $135.1,129.4,128.8,127.3,81.1,42.8,28.3$. The characterization data correspond to the reported values. ${ }^{6}$

Following the general procedure A, starting from tert-butyl 2-phenylacetate (51) (673 $\mathrm{mg}, 3.50$ mmol, 1.0 equiv.), the title compound $\mathbf{5 2}$ was obtained after purification by column chromatography on Biotage (Büchi flashpure cartridge $40 \mathrm{~g}$, Pentane:EtOAc 95:5) as a yellow oil (560 mg, $2.57 \mathrm{mmol}, 73 \%$ yield). ${ }^{1} \mathrm{H}$ NMR (400 MHz, Chloroform-d) $\delta 7.44-7.37$ (m, 2H, $\mathrm{ArH}), 7.37-7.32(\mathrm{~m}, 2 \mathrm{H}, \mathrm{ArH}), 7.21-7.13(\mathrm{~m}, 1 \mathrm{H}), 1.55\left(\mathrm{~s}, 9 \mathrm{H},{ }^{t} \mathrm{Bu}\right) ;{ }^{13} \mathrm{C}$ NMR (101 MHz, Chloroform- $d) \delta 164.6,128.9,126.4,125.8,124.2,82.2,28.5$. One carbon was not resolved. The characterization data correspond to the reported values. ${ }^{7}$

\footnotetext{
${ }^{4}$ H. M. L. Davies, T. Hansen, M. R. Churchill, J. Am. Chem. Soc. 2000, 122, 3063-3070.

${ }^{5}$ M. Gao, Y. Zhao, C. Zhong, S. Liu, P. Liu, Q. Yin, L. Hu, Org. Lett. 2019, 21, 5679-5684.

${ }^{6}$ T. Hama, J. F. Hartwig, Org. Lett. 2008, 10, 1549-1552.

${ }^{7}$ M. Buchsteiner, L. Martinez-Rodriguez, P. Jerabek, I. Pozo, M. Patzer, N. Nöthling, C. W. Lehmann, A. Fürstner, Chem. Eur. J. 2020, 26, 2509-2515.
} 


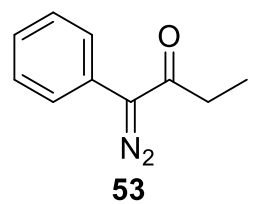

Following the general procedure A, starting from 1-phenylbutan-2-one $(0.75 \mathrm{~mL}, 5.0 \mathrm{mmol}, 1.0$ equiv.), the title compound $\mathbf{5 3}$ was obtained after purification by column chromatography on Biotage (Büchi flashpure cartridge $40 \mathrm{~g}$, Pentane:EtOAc 90:10) as a yellow solid (468 mg, 2.69 mmol, $54 \%$ yield). ${ }^{1} \mathbf{H}$ NMR (400 MHz, Chloroform-d) 7.52 (d, $\left.J=7.6 \mathrm{~Hz}, 2 \mathrm{H}, \mathrm{ArH}\right), 7.41$ (t, $J$ $=7.6 \mathrm{~Hz}, 2 \mathrm{H}, \mathrm{ArH}), 7.28(\mathrm{t}, J=7.6 \mathrm{~Hz}, 1 \mathrm{H}, \mathrm{ArH}), 2.63\left(\mathrm{q}, J=7.6 \mathrm{~Hz}, 2 \mathrm{H}, \mathrm{CH}_{2} \mathrm{CH}_{3}\right), 1.21(\mathrm{t}, J=$ $\left.7.6 \mathrm{~Hz}, 3 \mathrm{H}, \mathrm{CH}_{2} \mathrm{CH}_{3}\right) ;{ }^{13} \mathbf{C}$ NMR (101 MHz, Chloroform-d) $\delta$ 193.7, 129.1, 127.0, 126.0, 124.1, $32.6,8.7$. One carbon was not resolved. The characterization data correspond to the reported values. ${ }^{8}$

(1-Diazo-2,2,2-trifluoroethyl)benzene (56)

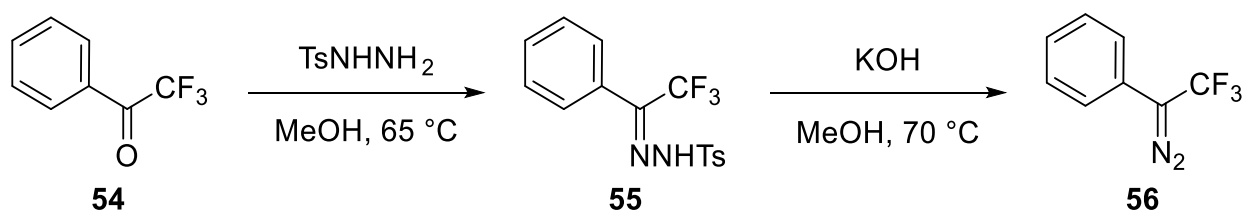

Following a reported procedure, ${ }^{9} \mathrm{TsNHNH}_{2}(2.24 \mathrm{~g}, 12.0 \mathrm{mmol}, 1.0$ equiv) was stirred in $\mathrm{MeOH}$ $(8 \mathrm{~mL})$ at reflux until complete dissolution. Then the reaction vessel was cooled to room temperature and 2,2,2-trifluoro-1-phenylethanone $(\mathbf{5 4})(1.69 \mathrm{~mL}, 12.0 \mathrm{mmol}, 1.0$ equiv.) was added in one portion. The resulting reaction mixture was then stirred at $65{ }^{\circ} \mathrm{C}$ for 12 hours, after which it was cooled to $0{ }^{\circ} \mathrm{C}$, leading to the precipitation of the desired product. The latter was collected by vacuum filtration and washed with pentane, leading to 55 (1.85 g, $5.40 \mathrm{mmol}, 45 \%$ yield $)$ as a white solid. ${ }^{1} \mathbf{H}$ NMR (400 MHz, Chloroform-d) $\delta 7.95$ (s, 1H, NH) $7.86-7.81$ (m, 2H, ArH), $7.59-7.48(\mathrm{~m}, 3 \mathrm{H}, \operatorname{Ar} H), 7.37(\mathrm{~d}, J=8.0 \mathrm{~Hz}, 2 \mathrm{H}, \operatorname{ArH}), 7.25(\mathrm{~d}, J=8.0 \mathrm{~Hz}, 2 \mathrm{H}, \operatorname{ArH}), 2.47$ (s, $\left.3 \mathrm{H}, \mathrm{CH}_{3}\right) .{ }^{13} \mathrm{C}$ NMR (101 MHz, Chloroform-d) $\delta 145.2,141.5$ (q, $\left.J=35.7 \mathrm{~Hz}\right), 134.7,131.6$, $130.3,129.9,128.6,128.2,125.3,120.2(\mathrm{q}, J=273 \mathrm{~Hz}), 21.9$. The characterization data correspond to the reported values. ${ }^{9}$

Following a reported procedure, ${ }^{9}$ 4-methyl-N'-(2,2,2-trifluoro-1-phenylethylidene)benzenesulfon -ohydrazide (55) (1.85 g, $5.40 \mathrm{mmol}, 1.0$ equiv.) was added to a solution of $\mathrm{KOH}$ (606 $\mathrm{mg}, 10.8$ mmol, 2.0 equiv.) in $\mathrm{MeOH}(13.5 \mathrm{~mL})$ and the resulting suspension was heated to reflux for two hours. The reaction was then cooled to room temperature and diluted with distilled water $(25 \mathrm{~mL})$. It was then extracted with DCM $(3 \times 20 \mathrm{~mL})$ and washed with a saturated aqueous solution of $\mathrm{NaHCO}_{3}(20 \mathrm{~mL})$ and brine $(20 \mathrm{~mL})$. The combined organic layers were dried over $\mathrm{MgSO}_{4}$, filtered, and concentrated under reduced pressure. The crude residue was purified by column chromatography on Biotage (Büchi flashpure cartridge $40 \mathrm{~g}$, Pentane) leading to the title compound

${ }^{8}$ H. Tomioka, H. Okuno, S. Hondo, Y. Izawa, J. Am. Chem. Soc. 1980, 102, 7123-7125.

${ }^{9}$ E. Emer, J. Twilton, M. Tredwell, S. Calderwood, T. L. Collier, B. Liégault, M. Taillefer, V. Gouverneur, Org. Lett. 2014, 16, 6004-6007. 
56 (352 mg, 1.89 mmol, $35 \%$ yield) as a volatile red liquid. ${ }^{1} \mathbf{H}$ NMR (400 MHz, Chloroform- $\boldsymbol{d}$ ) $\delta 7.46$ - $7.38(\mathrm{~m}, 2 \mathrm{H}, \mathrm{ArH}), 7.25$ - $7.19(\mathrm{~m}, 1 \mathrm{H}, \mathrm{ArH}), 7.17-7.08(\mathrm{~m}, 2 \mathrm{H}, \mathrm{Ar} H) ;{ }^{\mathbf{1 3}} \mathbf{C}$ NMR $(\mathbf{1 0 1}$ MHz, Chloroform- $d$ ) $\delta 129.4,126.4(\mathrm{q}, J=270 \mathrm{~Hz}), 125.9,123.2,122.7$. One carbon was not resolved. The characterization data correspond to the reported values. ${ }^{9}$

Ethyl 2-(4-bromophenyl)-2-diazoacetate (57)

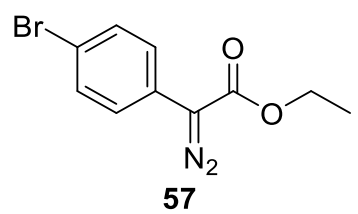

Following the general procedure A, starting from ethyl 2-(4-bromophenyl)acetate (1.30 g, 5.35 mmol, 1.0 equiv.), the title compound $\mathbf{5 7}$ was obtained after purification by column chromatography on Biotage (Büchi flashpure cartridge $40 \mathrm{~g}$, Pentane:EtOAc 90:10) as an orange solid (1.33 g, 4.94 mmol, $92 \%$ yield). ${ }^{1} \mathbf{H}$ NMR (400 MHz, Chloroform- $d$ ) $\delta 7.55-7.45$ (m, 2H, $\mathrm{ArH}), 7.40-7.32(\mathrm{~m}, 2 \mathrm{H}, \mathrm{Ar} H), 4.33\left(\mathrm{q}, J=7.1 \mathrm{~Hz}, 2 \mathrm{H}, \mathrm{CO}_{2} \mathrm{CH}_{2} \mathrm{CH}_{3}\right), 1.34(\mathrm{t}, J=7.1 \mathrm{~Hz}, 3 \mathrm{H}$, $\left.\mathrm{CO}_{2} \mathrm{CH}_{2} \mathrm{CH}_{3}\right) ;{ }^{13} \mathrm{C}$ NMR (101 MHz, Chloroform-d) $\delta 165.3,132.1,125.4,125.2,119.5,61.3$, 14.7. One carbon was not resolved. The characterization data correspond to the reported values. ${ }^{10}$

Methyl 2-diazo-2-(4-methoxyphenyl)acetate (58)

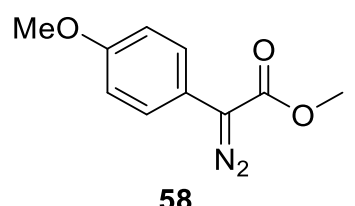

Following the general procedure A, starting from methyl 2-(4-methoxyphenyl)acetate (1.20 g, 6.66 mmol, 1.0 equiv.), the title compound $\mathbf{5 8}$ was obtained after purification by column chromatography on Biotage (Büchi flashpure cartridge $40 \mathrm{~g}$, Pentane:EtOAc 95:5) as an orange solid (838 mg, $4.06 \mathrm{mmol}, 61 \%$ yield). ${ }^{1} \mathbf{H}$ NMR (400 MHz, Chloroform-d) $\delta 7.42-7.34$ (m, 2H, $\mathrm{ArH}), 6.99-6.87(\mathrm{~m}, 2 \mathrm{H}, \mathrm{ArH}), 3.85\left(\mathrm{~s}, 3 \mathrm{H}, \mathrm{CO}_{2} \mathrm{CH}_{3}\right), 3.81\left(\mathrm{~s}, 3 \mathrm{H}, \mathrm{OCH}_{3}\right) ;{ }^{13} \mathbf{C}$ NMR (101 MHz, Chloroform- $d$ ) $\delta 166.2,158.2,126.1,116.7,114.5,55.5,51.7$. One carbon was not resolved. The characterization data correspond to the reported values. ${ }^{4}$

\section{Methyl 2-diazo-2-(p-tolyl)acetate (59)}

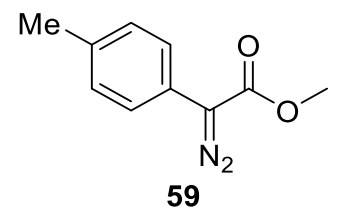

Following the general procedure A, starting from methyl 2-(p-tolyl)acetate $(800 \mathrm{mg}, 4.87 \mathrm{mmol}$, 1.0 equiv.), the title compound $\mathbf{5 9}$ was obtained after purification by column chromatography on

${ }^{10}$ N. D. Hahn, M. Nieger, K. H. Dötz, J. Organomet. Chem. 2004, 16, 2662-2673. 
Biotage (Büchi flashpure cartridge $40 \mathrm{~g}$, Pentane:EtOAc 95:5) as a red liquid (840 mg, $4.42 \mathrm{mmol}$, $91 \%$ yield). ${ }^{1} \mathbf{H}$ NMR (400 MHz, Chloroform-d) $\delta 7.42-7.32$ (m, 2H, ArH), 7.23 - 7.17 (m, 2H, $\mathrm{ArH}), 3.86$ (s, 3H, $\left.\mathrm{CO}_{2} \mathrm{CH}_{3}\right), 2.35\left(\mathrm{~s}, 3 \mathrm{H}, \mathrm{CH}_{3}\right) ;{ }^{13} \mathrm{C}$ NMR (101 MHz, Chloroform-d) $\delta 165.9$, $135.8,129.7,124.2,122.1,52.0,21.1$. One carbon was not resolved. The characterization data correspond to the reported values. ${ }^{4}$

Methyl 2-diazo-2-(2-fluorophenyl)acetate (60)

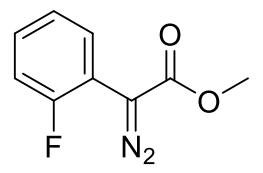

60

Following the general procedure A, starting from methyl 2-(2-fluorophenyl)acetate ( $800 \mathrm{mg}, 4.76$ mmol, 1.0 equiv.), the title compound $\mathbf{6 0}$ was obtained after purification by column chromatography on Biotage (Büchi flashpure cartridge $40 \mathrm{~g}$, Pentane:EtOAc 95:5) as a yellow oil (900 mg, 4.64 mmol, $97 \%$ yield). ${ }^{1}$ H NMR (400 MHz, Chloroform-d) $\delta 7.69$ (td, $J=7.8,2.0 \mathrm{~Hz}$, 1H, ArH), $7.29-7.15$ (m, 2H, ArH), 7.08 (ddd, $J=11.2,8.0,1.7 \mathrm{~Hz}, 1 \mathrm{H}, \operatorname{ArH}), 3.86(\mathrm{~s}, 3 \mathrm{H}$, $\left.\mathrm{CO}_{2} \mathrm{CH}_{3}\right) ;{ }^{13} \mathrm{C}$ NMR (101 MHz, Chloroform- $d$ ) $\delta 165.9,158.7(\mathrm{~d}, J=248.9 \mathrm{~Hz}), 129.8,128.9(\mathrm{~d}$, $J=8.4 \mathrm{~Hz}), 124.6(\mathrm{~d}, J=3.5 \mathrm{~Hz}), 116.1(\mathrm{~d}, J=21.3 \mathrm{~Hz}), 113.9(\mathrm{~d}, J=11.9 \mathrm{~Hz}), 52.4$. One carbon was not resolved. The characterization data correspond to the reported values. ${ }^{11}$

\section{Methyl 2-(2-chloro-4-fluorophenyl)-2-diazoacetate (61)}

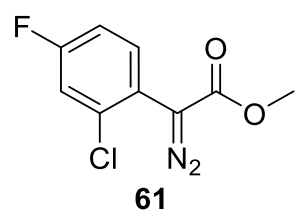

Following the general procedure A, starting from methyl 2-(2-chloro-4-fluorophenyl)acetate (900 mg, 4.44 mmol, 1.0 equiv.), the title compound $\mathbf{6 1}$ was obtained after purification by column chromatography on Biotage (Büchi flashpure cartridge $40 \mathrm{~g}$, Pentane:EtOAc 95:5) as a yellow oil (941 mg, 4.12 mmol, $93 \%$ yield). $\mathbf{R}_{f} 0.55$ (Pentane:EtOAc 95:5); ${ }^{1} \mathbf{H}$ NMR (400 MHz, Chloroform-d) $\delta 7.52(\mathrm{dd}, J=8.8,6.0 \mathrm{~Hz}, 1 \mathrm{H}, \mathrm{ArH}), 7.19(\mathrm{dd}, J=8.4,2.7 \mathrm{~Hz}, 1 \mathrm{H}, \mathrm{ArH}), 7.06$ (ddd, $J=8.8,7.8,2.7 \mathrm{~Hz}, 1 \mathrm{H}, \mathrm{ArH}), 3.84\left(\mathrm{~s}, 3 \mathrm{H}, \mathrm{CO}_{2} \mathrm{CH}_{3}\right) ;{ }^{\mathbf{1 3}} \mathbf{C}$ NMR (101 MHz, Chloroform-d) $\delta 166.0,162.5(\mathrm{~d}, J=252.4 \mathrm{~Hz}), 135.1(\mathrm{~d}, J=10.6 \mathrm{~Hz}), 133.8(\mathrm{~d}, J=9.1 \mathrm{~Hz}), 120.2(\mathrm{~d}, J=3.7$ $\mathrm{Hz}), 117.5(\mathrm{~d}, J=25.1 \mathrm{~Hz}), 115.0(\mathrm{~d}, J=21.6 \mathrm{~Hz}), 61.1,52.5 ;{ }^{19}$ F NMR (376 MHz, Chloroformd) $\delta$-110.6; IR $\widetilde{\boldsymbol{v}}_{\max } 3082(\mathrm{w}), 3001(\mathrm{w}), 2956(\mathrm{w}), 2100$ (s), 1703 (s), 1598 (m), 1495 (s), 1442 (m), $1394(\mathrm{~m}), 1347(\mathrm{~m}), 1290$ (s), 1258 (s), 1201 (s), 1161 (m), 1066 (m), 1023 (m), 920 (m), 891 $(\mathrm{m}), 865(\mathrm{~m}), 822(\mathrm{~m}), 743(\mathrm{~m}), 694(\mathrm{w}), 607$ (m); HRMS (ESI) calcd for $\mathrm{C}_{9} \mathrm{H}_{7} \mathrm{ClFN}_{2} \mathrm{O}_{2}{ }^{+}[\mathrm{M}+\mathrm{H}]^{+}$ 229.0175; found 229.0170.

11 T. C. Maier, G. C. Fu, J. Am. Chem. Soc. 2006, 128, 4594-4595. 
Methyl 2-diazo-2-(thiophen-3-yl)acetate (62)

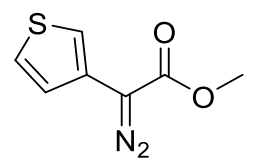

62

Following the general procedure A, starting from methyl 2-(thiophen-3-yl)acetate (830 mg, 5.31 mmol, 1.0 equiv.), the title compound $\mathbf{6 2}$ was obtained after purification by column chromatography on Biotage (Büchi flashpure cartridge $40 \mathrm{~g}$, Pentane:EtOAc 95:5) as a red liquid (758 mg, 4.16 mmol, $78 \%$ yield). ${ }^{1} \mathbf{H}$ NMR (400 MHz, Chloroform-d) $\delta 7.44-7.35$ (m, 2H, HetArH), $7.04(\mathrm{dd}, J=5.0,1.4 \mathrm{~Hz}, 1 \mathrm{H}, \operatorname{Het} \mathrm{Ar} H), 3.87\left(\mathrm{~s}, 3 \mathrm{H}, \mathrm{CO}_{2} \mathrm{CH}_{3}\right) ;{ }^{\mathbf{1 3}} \mathbf{C} \mathbf{N M R}(\mathbf{1 0 1} \mathbf{~ M H z}$, Chloroform-d) $\delta 165.9,126.4,123.8,123.4,117.6,52.1$. One carbon was not resolved. The characterization data correspond to the reported values. ${ }^{12}$

Ethyl 2-diazo-2-(naphthalen-2-yl)acetate (63)

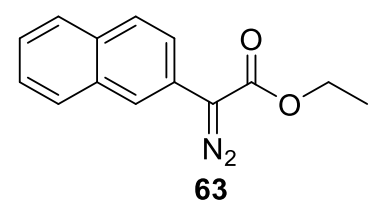

Following the general procedure A, starting from ethyl 2-(naphthalen-2-yl)acetate (952 mg, 4.45 mmol, 1.0 equiv.), the title compound $\mathbf{6 3}$ was obtained after purification by column chromatography on Biotage (Büchi flashpure cartridge $40 \mathrm{~g}$, Pentane:EtOAc 95:5) as an orange solid (897 mg, $3.73 \mathrm{mmol}, 84 \%$ yield). ${ }^{1} \mathbf{H}$ NMR (400 MHz, Chloroform- $d$ ) $\delta 8.02$ (d, $J=1.9$ $\mathrm{Hz}, 1 \mathrm{H}, \operatorname{Ar} H), 7.85(\mathrm{~d}, J=8.7 \mathrm{~Hz}, 1 \mathrm{H}, \operatorname{Ar} H), 7.80(\mathrm{~d}, J=8.8 \mathrm{~Hz}, 2 \mathrm{H}, \operatorname{Ar} H), 7.55(\mathrm{dd}, J=8.7,2.0$ $\mathrm{Hz}, 1 \mathrm{H}, \mathrm{Ar} H), 7.51-7.40(\mathrm{~m}, 2 \mathrm{H}, \mathrm{Ar} H), 4.38\left(\mathrm{q}, J=7.1 \mathrm{~Hz}, 2 \mathrm{H}, \mathrm{CH}_{2} \mathrm{CH}_{3}\right), 1.38(\mathrm{t}, J=7.1 \mathrm{~Hz}$, $\left.3 \mathrm{H}, \mathrm{CH}_{2} \mathrm{CH}_{3}\right)$; ${ }^{13} \mathbf{C}$ NMR (101 MHz, Chloroform-d) $\delta$ 165.5, 133.8, 131.6, 128.8, 127.8, 127.8, $126.7,125.9,123.0,122.7,122.1,61.2,14.7$. One carbon was not resolved. The characterization data correspond to the reported values. ${ }^{13}$

Methyl 2-diazo-2-(naphthalen-1-yl)acetate (64)

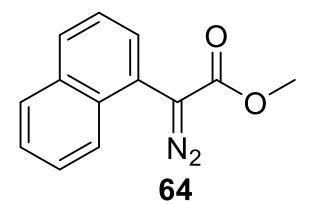

Following the general procedure A, starting from methyl 2-(naphthalen-1-yl)acetate (2.00 g, 9.99 mmol, 1.0 equiv.), the title compound $\mathbf{6 4}$ was obtained after purification by column chromatography on Biotage (Büchi flashpure cartridge $40 \mathrm{~g}$, Pentane:EtOAc 95:5) as a red liquid (2.05 g, 9.04 mmol, $90 \%$ yield). ${ }^{1}$ H NMR (400 MHz, Chloroform-d) $\delta 7.94-7.85$ (m, 3H, ArH),

${ }^{12}$ H. M. L. Davies, R. J. Townsend, J. Org. Chem. 2001, 66, 6595-6603.

${ }^{13}$ G. Chen, J. Song, Y. Yu, X. Luo, C. Li, X. Huang, Chem. Sci. 2016, 7, 1786-1790. 
7.66 - 7.51 (m, 4H, ArH), $3.86\left(\mathrm{~s}, 3 \mathrm{H}, \mathrm{CO}_{2} \mathrm{CH}_{3}\right)$; ${ }^{\mathbf{1 3}} \mathrm{C}$ NMR (101 MHz, Chloroform-d) $\delta$ 166.8, $134.2,131.5,129.7,129.6,128.7,126.4,126.2,125.3,124.4,122.2,52.1$. One carbon was not resolved. The characterization data correspond to the reported values. ${ }^{12}$

\subsection{Synthesis of Cyclopropenes}

General procedure B: Synthesis of cyclopropenes from diazo compounds.

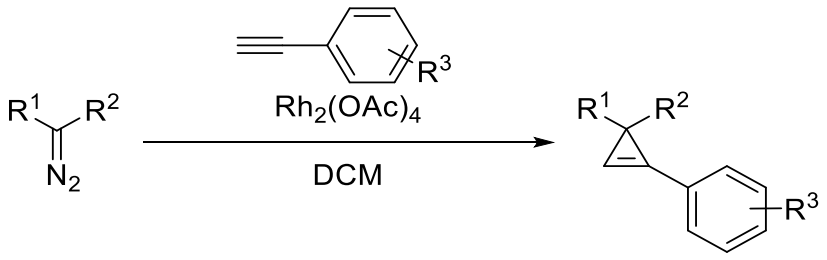

Following a modified procedure, ${ }^{14}$ the diazo compound was dissolved in DCM $(0.5 \mathrm{M})$ and the resulting solution was added via syringe pump to a suspension of $\mathrm{Rh}_{2}(\mathrm{OAc})_{4}(0.01$ equiv. $)$ and the indicated acetylene (3.0 equiv.) in DCM $(0.8 \mathrm{M})$ at room temperature over 10 hours. After the addition was complete, the reaction mixture was allowed to stir for another 10 hours. The reaction mixture was then filtered through a small pad of silica eluting with $\mathrm{CH}_{2} \mathrm{Cl}_{2}$ and the filtrate was concentrated under reduced pressure. The crude residue was purified by column chromatography with the indicated solvents.

\section{Dimethyl 2-phenylcycloprop-2-ene-1,1-dicarboxylate (1)}

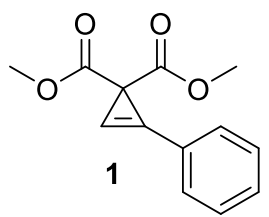

Following the general procedure B, starting from dimethyl 2-diazomalonate (42) (5.10 g, 32.0 mmol, 1.0 equiv.) and phenylacetylene (10.54 mL, $96.00 \mathrm{mmol}, 3.0$ equiv.), the title compound 1 was obtained after purification by column chromatography $\left(\mathrm{SiO}_{2}\right.$, Pentane:Et $\mathrm{E}_{2} \mathrm{O}$ 80:20) as a pale yellow solid (5.08 g, 21.9 mmol, $68 \%$ yield). ${ }^{1}$ H NMR (400 MHz, Chloroform-d) $\delta 7.67-7.60$ $(\mathrm{m}, 2 \mathrm{H}, \mathrm{ArH}), 7.48-7.41(\mathrm{~m}, 3 \mathrm{H}, \mathrm{ArH}), 6.89(\mathrm{~s}, 1 \mathrm{H}, \mathrm{C}=\mathrm{CH}), 3.74\left(\mathrm{~s}, 6 \mathrm{H}, \mathrm{CH}_{3}\right) ;{ }^{\mathbf{1 3}} \mathbf{C} \mathbf{N M R}(\mathbf{1 0 1}$ MHz, Chloroform-d) 171.3, 130.8, 130.4, 128.8, 123.5, 112.2, 95.1, 52.3, 32.8. The characterization data correspond to the reported values. ${ }^{15}$

\section{Dibenzyl 2-phenylcycloprop-2-ene-1,1-dicarboxylate (65)}

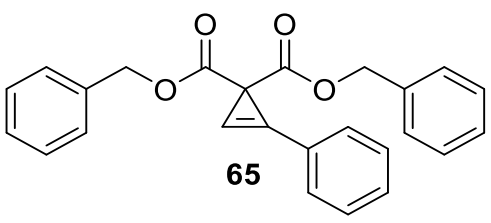

${ }^{14}$ S. Chuprakov, M. Rubin, V. Gevorgyan, J. Am. Chem. Soc. 2005, 127, 3714-3715.

${ }^{15}$ L.-A. Liao, F. Zhang, N. Yan, J. A. Golen, J. M. Fox, Tetrahedron 2004, 60, 1803-1816. 
Following the general procedure B, starting from dibenzyl 2-diazomalonate (44) (889 mg, 2.86 mmol, 1.0 equiv.) and phenylacetylene $(0.95 \mathrm{~mL}, 8.5 \mathrm{mmol}, 3.0$ equiv. $)$, the title compound 65 was obtained after purification by column chromatography $\left(\mathrm{SiO}_{2}\right.$, Pentane:Et $\mathrm{Et}_{2} \mathrm{O} 80: 20$ to $\left.70: 30\right)$ as a pale yellow solid (738 mg, $1.92 \mathrm{mmol}, 67 \%$ yield). ${ }^{1} \mathbf{H}$ NMR (400 MHz, Chloroform- $d$ ) $\delta 7.64-$ $7.58(\mathrm{~m}, 2 \mathrm{H}, \mathrm{Ar} H), 7.44-7.40(\mathrm{~m}, 3 \mathrm{H}, \mathrm{Ar} H), 7.31-7.26(\mathrm{~m}, 10 \mathrm{H}, \mathrm{Ar} H), 6.91(\mathrm{~s}, 1 \mathrm{H}, \mathrm{C}=\mathrm{CH})$, $5.18\left(\mathrm{~s}, 4 \mathrm{H}, \mathrm{CH}_{2}\right){ }^{13} \mathbf{C}$ NMR (101 MHz, Chloroform-d) $\delta$ 170.7, 135.9, 130.7, 130.5, 129.0, 128.6, $128.2,128.0,124.1,112.5,95.3,67.0,33.5$. The characterization data correspond to the reported values. $^{16}$

Bis(2,2,2-trifluoroethyl) 2-phenylcycloprop-2-ene-1,1-dicarboxylate (66)

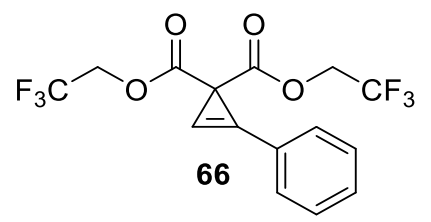

Following the general procedure B, starting from bis(2,2,2-trifluoroethyl) 2-diazomalonate (48) (935 mg, $3.18 \mathrm{mmol}, 1.0$ equiv.) and phenylacetylene (1.05 mL, $9.54 \mathrm{mmol}, 3.0$ equiv), the title compound 66 was obtained after purification by column chromatography $\left(\mathrm{SiO}_{2}, \mathrm{Pentane}_{\mathrm{Et}} \mathrm{O}\right.$ 90:10 to 80:20) as a pale yellow oil $\left(542 \mathrm{mg}, 1.47 \mathrm{mmol}, 46 \%\right.$ yield). $\mathbf{R}_{f} 0.55$ (Pentane: $\mathrm{Et}_{2} \mathrm{O}$ 80:20). ${ }^{1} \mathrm{H}$ NMR (400 MHz, Chloroform- $\left.\boldsymbol{d}\right) \delta 7.66-7.57$ (m, 2H, $\left.\mathrm{Ar} H\right), 7.51-7.44(\mathrm{~m}, 3 \mathrm{H}$, $\mathrm{ArH}), 6.92(\mathrm{~s}, 1 \mathrm{H}, \mathrm{C}=\mathrm{CH}), 4.53$ (qd, $J=8.3,6.3 \mathrm{~Hz}, 4 \mathrm{H}, \mathrm{CH})_{2}$ ) ${ }^{13} \mathbf{C}$ NMR (101 MHz, Chloroformd) $\delta 168.6,131.4,130.5,129.2,123.0,122.8(\mathrm{q}, J=277.3 \mathrm{~Hz}), 111.7,93.9,61.1(\mathrm{q}, J=37.0 \mathrm{~Hz})$, 32.5. The characterization data correspond to the reported values. ${ }^{17}$

Dimethyl 2-(4-bromophenyl)cycloprop-2-ene-1,1-dicarboxylate (67)<smiles>COC(=O)C1(C(=O)OC)CC1c1ccc(Br)cc1</smiles>

Following the general procedure B, starting from dimethyl 2-diazomalonate (42) (395 mg, 2.50 mmol, 1.0 equiv.) and 1-bromo-4-ethynylbenzene (1.36 g, $7.50 \mathrm{mmol}, 3.0$ equiv.), the title compound 67 was obtained after purification by column chromatography $\left(\mathrm{SiO}_{2}\right.$, Pentane:EtOAc 95:5 to 85:15) as a pale yellow oil (554 mg, $1.78 \mathrm{mmol}, 71 \%$ yield). ${ }^{1} \mathbf{H}$ NMR (400 MHz, Chloroform-d $) \delta 7.62-7.56(\mathrm{~m}, 2 \mathrm{H}, \mathrm{Ar} H), 7.51-7.46(\mathrm{~m}, 2 \mathrm{H}, \mathrm{Ar} H), 6.94(\mathrm{~s}, 1 \mathrm{H}, \mathrm{C}=\mathrm{CH}), 3.73$ (s, $\left.6 \mathrm{H}, \mathrm{CO}_{2} \mathrm{CH}_{3}\right) ;{ }^{13} \mathbf{C}$ NMR (101 MHz, Chloroform-d) $\delta$ 171.0, 132.4, 131.9, 125.4, 123.1, 111.7, $96.4,52.7,33.0$. The characterization data correspond to the reported values. ${ }^{18}$

\footnotetext{
${ }^{16}$ Y. Wang, E. A. F. Fordyce, F. Y. Chen, H. W. Lam, Angew. Chem. Int. Ed. 2008, 47, 7350 -7353.

${ }^{17}$ B. Muriel, A. Gagnebin, J. Waser, Chem. Sci. 2019, 10, 10716-10722.

${ }^{18}$ E. A. F. Fordyce, T. Luebbers, H. W. Lam, Org. Lett. 2008, 10, 3993-3996.
} 
Dimethyl 2-(4-(tert-butyl)phenyl)cycloprop-2-ene-1,1-dicarboxylate (68)

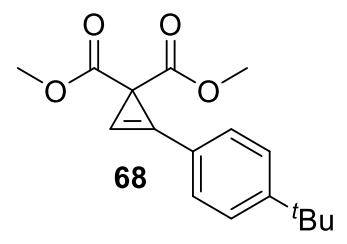

Following the general procedure B, starting from dimethyl 2-diazomalonate (42) (395 mg, 2.50 mmol, 1.0 equiv.) and 1-(tert-butyl)-4-ethynylbenzene ( $1.35 \mathrm{~mL}, 7.50 \mathrm{mmol}, 3.0$ equiv.), the title compound $\mathbf{6 8}$ was obtained after purification by column chromatography $\left(\mathrm{SiO}_{2}\right.$, Pentane:EtOAc 95:5 to 90:10) as an orange solid (517 mg, $1.79 \mathrm{mmol}, 72 \%$ yield). ${ }^{1} \mathbf{H}$ NMR (400 MHz, Chloroform-d) $\delta 7.58-7.54(\mathrm{~m}, 2 \mathrm{H}, \mathrm{Ar} H), 7.49-7.44(\mathrm{~m}, 2 \mathrm{H}, \mathrm{Ar} H), 6.82(\mathrm{~s}, 1 \mathrm{H}, \mathrm{C}=\mathrm{CH}), 3.73$ (s, 6H, $\left.\mathrm{OCH}_{3}\right), 1.33$ (s, 9H, $\left.{ }^{t} \mathrm{Bu}\right) ;{ }^{13} \mathbf{C}$ NMR (101 MHz, Chloroform-d) $\delta$ 171.4, 154.3, 130.3, $126.1,121.2,112.1,94.3,52.5,35.2,32.8,31.3$. The characterization data correspond to the reported values. ${ }^{19}$

Dimethyl 2-(4-methoxyphenyl)cycloprop-2-ene-1,1-dicarboxylate (69)

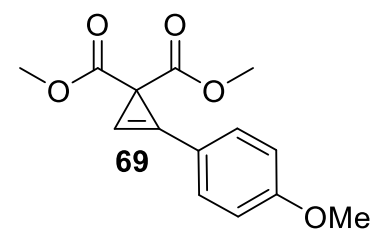

Following the general procedure B, starting from dimethyl 2-diazomalonate (42) (316 mg, 2.00 mmol, 1.0 equiv.) and 1-ethynyl-4-methoxybenzene (0.8 mL, $6 \mathrm{mmol}, 3.0$ equiv.), the title compound 69 was obtained after purification by column chromatography $\left(\mathrm{SiO}_{2}\right.$, Pentane:EtOAc 95:5 to 85:15) as a white solid (157 mg, $0.599 \mathrm{mmol}, 30 \%$ yield). ${ }^{1} \mathbf{H}$ NMR (400 MHz, Chloroform-d) $\delta 7.51-7.43(\mathrm{~m}, 2 \mathrm{H}, \mathrm{Ar} H), 6.95-6.87(\mathrm{~m}, 2 \mathrm{H}, \mathrm{Ar} H), 6.70(\mathrm{~s}, 1 \mathrm{H}, \mathrm{C}=\mathrm{C} H), 4.19$ (s, $\left.3 \mathrm{H}, \mathrm{OCH}_{3}\right), 3.83$ (s, 3H, $\left.\mathrm{CO}_{2} \mathrm{CH}_{3}\right), 3.82$ (s, $\left.3 \mathrm{H}, \mathrm{CO}_{2} \mathrm{CH}_{3}\right) ;{ }^{\mathbf{1 3}} \mathbf{C}$ NMR (101 MHz, Chloroformd) $\delta 163.6,161.5,159.1,143.8,124.5,122.9,114.4,104.6,93.1,58.3,55.5,51.5$. The characterization data correspond to the reported values. ${ }^{20}$

\section{Dimethyl 2-(3-fluorophenyl)cycloprop-2-ene-1,1-dicarboxylate (70)}

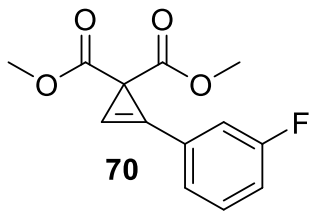

Following the general procedure B, starting from dimethyl 2-diazomalonate (42) (316 mg, 2.00 mmol, 1.0 equiv.) and 1-ethynyl-3-fluorobenzene ( $0.69 \mathrm{~mL}, 6.0 \mathrm{mmol}, 3.0$ equiv.), the title compound 70 was obtained after purification by column chromatography $\left(\mathrm{SiO}_{2}\right.$, Pentane:EtOAc

${ }^{19}$ Y. Liu, Q. Yu, S. Ma, Eur. J. Org. Chem. 2013, 3033-3040.

${ }^{20}$ Q. Ye, H. Ye, D. Cheng, X. Li, X. Xu, Tetrahedron Lett. 2018, 59, 2546-2549. 
90:10) as a pale yellow oil (282 mg, $1.13 \mathrm{mmol}, 56 \%$ yield). ${ }^{\mathbf{1}} \mathbf{H}$ NMR (400 MHz, Chloroformd) $\delta 7.44-7.40(\mathrm{~m}, 2 \mathrm{H}, \mathrm{ArH}), 7.33(\mathrm{dd}, J=9.0,2.5 \mathrm{~Hz}, 1 \mathrm{H}, \mathrm{ArH}), 7.18-7.11(\mathrm{~m}, 1 \mathrm{H}, \mathrm{ArH}), 6.96$ $(\mathrm{s}, 1 \mathrm{H}, \mathrm{C}=\mathrm{CH}), 3.75\left(\mathrm{~s}, 6 \mathrm{H}, \mathrm{OCH}_{3}\right) ;{ }^{13} \mathrm{C}$ NMR (101 MHz, Chloroform-d) $\delta 171.0,162.9(\mathrm{~d}, J=$ $248.0 \mathrm{~Hz}), 130.7(\mathrm{~d}, J=8.4 \mathrm{~Hz}), 126.3(\mathrm{~d}, J=3.1 \mathrm{~Hz}), 126.1(\mathrm{~d}, J=8.3 \mathrm{~Hz}), 117.9(\mathrm{~d}, J=21.2$ $\mathrm{Hz}), 117.1(\mathrm{~d}, J=22.7 \mathrm{~Hz}), 111.7(\mathrm{~d}, J=3.4 \mathrm{~Hz}), 97.0,52.7,33.2$. The characterization data correspond to the reported values. ${ }^{17}$

Dimethyl 2-(naphthalen-1-yl)cycloprop-2-ene-1,1-dicarboxylate (71)

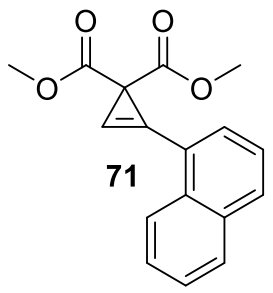

Following the general procedure B, starting from dimethyl 2-diazomalonate (42) (316 mg, 2.00 mmol, 1.0 equiv.) and 1-ethynylnaphthalene ( $0.85 \mathrm{~mL}, 6.0 \mathrm{mmol}, 3.0$ equiv.), the title compound 71 was obtained after purification by column chromatography $\left(\mathrm{SiO}_{2}\right.$, Pentane:EtOAc 90:10) as a pale yellow solid (288 mg, $1.02 \mathrm{mmol}, 51 \%$ yield). ${ }^{1} \mathbf{H}$ NMR (400 MHz, Chloroform-d) $\delta$ 8.39$8.34(\mathrm{~m}, 1 \mathrm{H}, \mathrm{ArH}), 7.98-7.91(\mathrm{~m}, 2 \mathrm{H}, \mathrm{ArH}), 7.79-7.76(\mathrm{~m}, 1 \mathrm{H}, \mathrm{ArH}), 7.70-7.54(\mathrm{~m}, 3 \mathrm{H}, \mathrm{ArH}), 7.22$ (s, 1H, C=CH), $3.75\left(\mathrm{~s}, 6 \mathrm{H}, \mathrm{CO}_{2} \mathrm{CH}_{3}\right) ;{ }^{13} \mathbf{C}$ NMR (101 MHz, Chloroform-d) $\delta 171.3,133.6,132.3$, 131.6, 131.0, 128.8, 127.7, 126.8, 125.6, 124.5, 120.5, 110.8, 97.5, 52.6, 31.5. The characterization data correspond to the reported values. ${ }^{18}$

Dimethyl 2-(2-(trifluoromethyl)phenyl)cycloprop-2-ene-1,1-dicarboxylate (72)

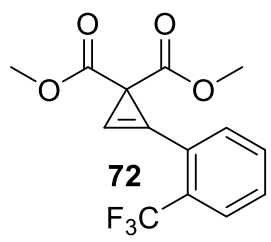

Following the general procedure B, starting from dimethyl 2-diazomalonate (42) (316 mg, 2.00 mmol, 1.0 equiv.) and 1-ethynyl-2-(trifluoromethyl)benzene ( $0.84 \mathrm{~mL}, 6.0 \mathrm{mmol}, 3.0$ equiv.), the title compound $\mathbf{7 2}$ was obtained after purification by column chromatography $\left(\mathrm{SiO}_{2}\right.$, Pentane:EtOAc 90:10) as a white solid (307 mg, $1.02 \mathrm{mmol}, 51 \%$ yield). $\mathbf{R}_{\boldsymbol{f}} 0.35$ (Pentane:EtOAc 80:20); m.p = 119-120 ${ }^{\circ} \mathrm{C} ;{ }^{1} \mathbf{H}$ NMR (400 MHz, Chloroform-d) $\delta 7.78(\mathrm{~d}, J=7.8 \mathrm{~Hz}, 1 \mathrm{H}, \operatorname{Ar} H$ ), $7.72-7.61(\mathrm{~m}, 2 \mathrm{H}, \mathrm{ArH}), 7.54(\mathrm{t}, J=7.6 \mathrm{~Hz}, 1 \mathrm{H}, \mathrm{ArH}), 7.10(\mathrm{~s}, 1 \mathrm{H}, \mathrm{C}=\mathrm{CH}), 3.74\left(\mathrm{~s}, 6 \mathrm{H}, \mathrm{CO}_{2} \mathrm{CH}_{3}\right)$; ${ }^{13}$ C NMR (101 MHz, Chloroform-d) $\delta$ 170.7, 132.8, 132.3, 130.5, 129.9 (q, $\left.J=32.3 \mathrm{~Hz}\right), 126.6$ $(\mathrm{q}, J=5.1 \mathrm{~Hz}), 123.6(\mathrm{q}, J=273.4 \mathrm{~Hz}), 121.9,109.2,101.2(\mathrm{q}, J=4.1 \mathrm{~Hz}), 52.5,33.2 ;{ }^{19} \mathbf{F ~ N M R}$ (376 MHz, Chloroform-d) $\delta$-62.7; IR $\widetilde{\boldsymbol{v}}_{\max } 3421$ (w), 3147 (w), 3040 (w), 2957 (w), 2847 (w), 1752 (s), 1442 (m), 1282 (s), 1174 (s), 1114 (s), 1066 (s), 981 (w), 909 (w), 778 (m), 743 (m), 701 (m), 642 (w); HRMS (ESI) calcd for $\mathrm{C}_{14} \mathrm{H}_{11} \mathrm{~F}_{3} \mathrm{NaO}_{4}{ }^{+}[\mathrm{M}+\mathrm{Na}]^{+} 323.0502$; found 323.0510. 
Dimethyl 2-butylcycloprop-2-ene-1,1-dicarboxylate (74)

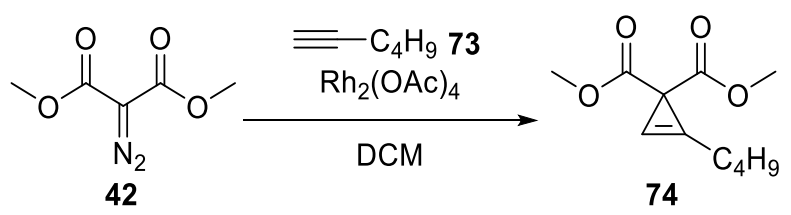

A solution of dimethyl 2-diazomalonate (42) (395 mg, $2.50 \mathrm{mmol}, 1.0$ equiv.) in DCM (1.75 mL) was added using a syringe pump over 15 hours to a stirred mixture of hex-1-yne (73) $(1.15 \mathrm{~mL}$, 10.0 mmol, 4.0 equiv.) and $\mathrm{Rh}_{2}(\mathrm{OAc})_{4}(11 \mathrm{mg}, 0.025 \mathrm{mmol}, 0.01$ equiv.) in DCM (2.0 mL). After the addition was complete, the mixture was stirred for additional 3 hours. The suspension was then filtered through a small pad of silica gel eluting with DCM, and the filtrate was evaporated under reduced pressure. The crude residue was purified by column chromatography $\left(\mathrm{SiO}_{2}\right.$, Pentane:EtOAc 90:10) affording the title compound 74 (240 $\mathrm{mg}, 1.13 \mathrm{mmol}, 45 \%$ yield) as a pale yellow oil. ${ }^{1} \mathbf{H}$ NMR (400 MHz, Chloroform-d) $\delta 6.35$ (t, $J=1.4 \mathrm{~Hz}, 1 \mathrm{H}, \mathrm{C}=\mathrm{CH}$ ), 3.71 (s, 6H, $\left.\mathrm{OCH}_{3}\right), 2.55\left(\mathrm{td}, J=7.4,1.4 \mathrm{~Hz}, 2 \mathrm{H}, \mathrm{CH}_{2} \mathrm{CH}_{2} \mathrm{CH}_{2} \mathrm{CH}_{3}\right), 1.60-1.53\left(\mathrm{~m}, 2 \mathrm{H}, \mathrm{CH}_{2} \mathrm{CH}_{2} \mathrm{CH}_{2} \mathrm{CH}_{3}\right)$, $1.44-1.32\left(\mathrm{~m}, 2 \mathrm{H}, \mathrm{CH}_{2} \mathrm{CH}_{2} \mathrm{CH}_{2} \mathrm{CH}_{3}\right), 0.91\left(\mathrm{t}, J=7.3 \mathrm{~Hz}, 3 \mathrm{H}, \mathrm{CH}_{2} \mathrm{CH}_{2} \mathrm{CH}_{2} \mathrm{CH}_{3}\right) ;{ }^{13} \mathbf{C ~ N M R}(\mathbf{1 0 1}$ MHz, Chloroform-d) 172.5, 114.8, 93.8, 52.8, 32.4, 29.1, 24.5, 22.5, 14.2. The characterization data correspond to the reported values. ${ }^{15}$

\section{Dimethyl 2-(trimethylsilyl)cycloprop-2-ene-1,1-dicarboxylate (76)}

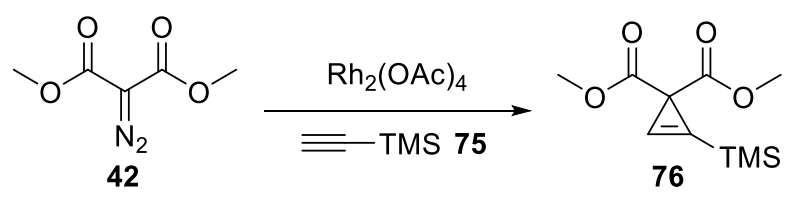

Following a modified procedure, ${ }^{21}$ a solution of dimethyl 2-diazomalonate (42) (0.79 g, $5.0 \mathrm{mmol}$, 1.0 equiv.) in ethynyltrimethylsilane (75) $(2 \mathrm{~mL})$ was added using a syringe pump over 18 hours to a refluxing stirred suspension of $\mathrm{Rh}_{2}(\mathrm{OAc})_{4}(22 \mathrm{mg}, 0.050 \mathrm{mmol}, 0.01$ equiv.) in ethynyltrimethylsilane (75) $(10 \mathrm{~mL})$. After the addition was complete, the reaction mixture was stirred at reflux for an additional 4 hours. The suspension was then filtered through a small pad of silica gel eluting with DCM, and the filtrate was evaporated under reduced pressure. The crude residue was purified by column chromatography $\left(\mathrm{SiO}_{2}\right.$, Pentane:EtOAc 90:10) affording the title compound $76\left(824 \mathrm{mg}, 3.61 \mathrm{mmol}, 72 \%\right.$ yield) as a colorless oil. ${ }^{1} \mathbf{H}$ NMR (400 $\mathbf{~ M H z}$, Chloroform-d) $\delta 7.05(\mathrm{~s}, 1 \mathrm{H}, \mathrm{C}=\mathrm{CH}), 3.70\left(\mathrm{~s}, 6 \mathrm{H}, \mathrm{OCH}_{3}\right), 0.25(\mathrm{~s}, 9 \mathrm{H}, \mathrm{TMS}) ;{ }^{13} \mathrm{C}$ NMR (101 MHz, Chloroform- $\boldsymbol{d}) \delta 172.4,113.5,110.8,52.8,30.8,-1.7$. The characterization data correspond to the reported values. ${ }^{21}$

Dimethyl cycloprop-2-ene-1,1-dicarboxylate (77)

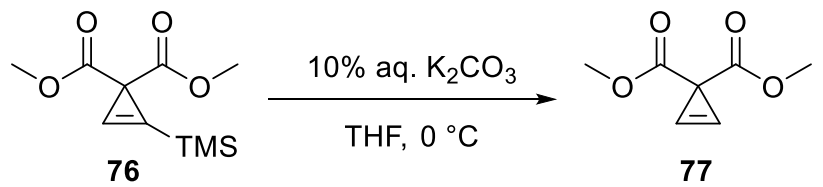

${ }^{21}$ S. Chuprakov, D. A. Malyshev, A. Trofimov, V. Gevorgyan, J. Am. Chem. Soc. 2007, 129, 14868-14869. 
Following a reported procedure, ${ }^{22}$ to a stirred solution of dimethyl 2-(trimethylsilyl)cycloprop-2ene-1,1-dicarboxylate (76) $(355 \mathrm{mg}, 1.55 \mathrm{mmol}, 1$ equiv.) in THF ( $3 \mathrm{~mL})$ was added dropwise a $10 \%$ aq. $\mathrm{K}_{2} \mathrm{CO}_{3}$ solution $(2 \mathrm{~mL})$. The reaction mixture was stirred for $30 \mathrm{~min}$ at $0{ }^{\circ} \mathrm{C}$ and $30 \mathrm{~min}$ at room temperature. Then brine $(5 \mathrm{~mL})$ was added and the layers were separated. The organic layer was further washed with brine $(2 \times 5 \mathrm{~mL})$, dried over $\mathrm{Na}_{2} \mathrm{SO}_{4}$, filtered and concentrated under reduced pressure. The crude residue was purified by column chromatography $\left(\mathrm{SiO}_{2}\right.$, Pentane:EtOAc 85:15) affording compound 77 (150 mg, $0.961 \mathrm{mmol}, 62 \%$ yield) as a colorless oil. ${ }^{1} \mathbf{H}$ NMR (400 MHz, Chloroform-d) $\delta 6.90(\mathrm{~s}, 2 \mathrm{H}, \mathrm{HC}=\mathrm{CH}), 3.73\left(\mathrm{~s}, 6 \mathrm{H}, \mathrm{OCH}_{3}\right) ;{ }^{13} \mathbf{C}$ NMR (101 MHz, Chloroform- $d$ ) $\delta 171.9,102.5,52.7,30.3$. The characterization data correspond to the reported values. ${ }^{22}$

\section{Methyl 1,2-diphenylcycloprop-2-ene-1-carboxylate (39)}

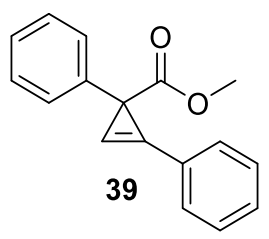

Following the general procedure B, starting from methyl 2-diazo-2-phenylacetate (49) (700 mg, $3.97 \mathrm{mmol}, 1.0$ equiv.) and phenylacetylene (1.31 mL, $11.9 \mathrm{mmol}, 3.0$ equiv.), the title compound 39 was obtained after purification by column chromatography on Biotage (Büchi flashpure cartridge $40 \mathrm{~g}$, Pentane:EtOAc 90:10) as a pale yellow oil (667 mg, $2.66 \mathrm{mmol}, 67 \%$ yield). ${ }^{1} \mathbf{H}$ NMR (400 MHz, Chloroform-d) $\delta 7.65-7.60$ (m, 2H, $\mathrm{ArH}$ ), $7.45-7.37$ (m, 5H, ArH), $7.33-$ $7.26(\mathrm{~m}, 2 \mathrm{H}, \mathrm{Ar} H), 7.23-7.18(\mathrm{~m}, 2 \mathrm{H}, \mathrm{ArH} \& \mathrm{C}=\mathrm{CH}), 3.72\left(\mathrm{~s}, 3 \mathrm{H}, \mathrm{CO}_{2} \mathrm{CH}_{3}\right) ;{ }^{13} \mathbf{C}$ NMR $(\mathbf{1 0 1}$ MHz, Chloroform- $d$ ) $\delta 175.2,141.0,130.1,130.1,129.0,128.3,128.2,126.7,125.6,117.5,100.5$, $52.4,33.7$. The characterization data correspond to the reported values. ${ }^{15}$

\section{Tert-butyl 1,2-diphenylcycloprop-2-ene-1-carboxylate (78)}

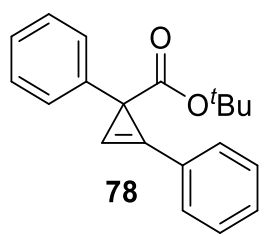

Following the general procedure B, starting from tert-butyl 2-diazo-2-phenylacetate (52) $560 \mathrm{mg}$, $2.57 \mathrm{mmol}, 1.0$ equiv.) and phenylacetylene ( $0.85 \mathrm{~mL}, 7.7 \mathrm{mmol}, 3.0$ equiv.), the title compound 78 was obtained after purification by column chromatography on Biotage (Büchi flashpure cartridge $40 \mathrm{~g}$, Pentane:EtOAc 85:15) as an orange oil (310 mg, $1.06 \mathrm{mmol}, 41 \%$ yield). $\mathbf{R}_{f} 0.36$ (Pentane:EtOAc 80:20); ${ }^{\mathbf{1}} \mathbf{H}$ NMR (400 MHz, Chloroform- $d$ ) $\delta 7.66$ - 7.59 (m, 2H, ArH), $7.45-$ $7.36(\mathrm{~m}, 5 \mathrm{H}, \operatorname{Ar} H), 7.29-7.25(\mathrm{~m}, 2 \mathrm{H}, \mathrm{Ar} H), 7.21-7.17$ (m, 2H, $\mathrm{ArH} \& \mathrm{C}=\mathrm{CH}), 1.44(\mathrm{~s}, 9 \mathrm{H}$, $\left.{ }^{t} \mathrm{Bu}\right) ;{ }^{13} \mathrm{C}$ NMR (101 MHz, Chloroform- $d$ ) $\delta$ 173.9, 141.7, 129.9, 129.9, 128.9, 128.3, 128.0, 126.3, 126.0, 117.6, 100.9, 80.8, 34.8, 28.2; IR $\widetilde{v}_{\max } 3134$ (w), 3059 (w), 3026 (w), 2932 (w), 1953

\footnotetext{
${ }^{22}$ K. Krämer, P. Leong, M. Lautens, Org. Lett. 2011, 13, 819-821.
} 
(w), 1711 (s), 1600 (w), 1491 (m), 1449 (m), 1390 (w), 1367 (m), 1247 (m), 1156 (s), 1074 (w), 1031 (w), 984 (m), 915 (w), 848 (m), 764 (m), 697 (s); HRMS (ESI) calcd for $\mathrm{C}_{20} \mathrm{H}_{20} \mathrm{NaO}_{2}^{+}[\mathrm{M}+\mathrm{Na}]^{+} 315.1356$; found 315.1355.

\section{1-(1,2-Diphenylcycloprop-2-en-1-yl)propan-1-one (79)}

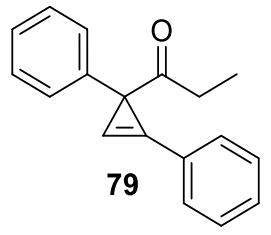

Following the general procedure B, starting from 1-diazo-1-phenylbutan-2-one (53) (468 mg, 2.69 mmol, 1.0 equiv.) and phenylacetylene ( $0.89 \mathrm{~mL}, 8.1 \mathrm{mmol}, 3.0$ equiv.), the title compound 79 was obtained after purification by column chromatography on Biotage (Büchi flashpure cartridge $40 \mathrm{~g}$, Pentane:EtOAc 95:5) as a yellow oil $\left(280 \mathrm{mg}, 1.13 \mathrm{mmol}, 39 \%\right.$ yield, $92 \%$ purity). $\mathbf{R}_{f} 0.38$ (Pentane:EtOAc 90:10); ${ }^{\mathbf{1}} \mathbf{H}$ NMR (400 MHz, Chloroform-d) $\delta 7.59-7.53$ (m, 2H, ArH), $7.41-$ $7.34(\mathrm{~m}, 3 \mathrm{H}, \mathrm{ArH}), 7.31-7.27(\mathrm{~m}, 2 \mathrm{H}, \mathrm{ArH}), 7.26-7.20(\mathrm{~m}, 3 \mathrm{H}, \mathrm{ArH} \& \mathrm{C}=\mathrm{CH}), 7.18-7.12(\mathrm{~m}$, $1 \mathrm{H}, \mathrm{ArH}), 2.39\left(\mathrm{dq}, J=17.7,7.3 \mathrm{~Hz}, 1 \mathrm{H}, \mathrm{CH}_{2}\right), 2.24\left(\mathrm{dq}, J=17.7,7.2 \mathrm{~Hz}, 1 \mathrm{H}, \mathrm{CH}_{2}\right), 0.96(\mathrm{t}, J=$ $\left.7.3 \mathrm{~Hz}, 3 \mathrm{H}, \mathrm{CH}_{3}\right) ;{ }^{13} \mathbf{C}$ NMR (101 MHz, Chloroform-d) $\delta 212.1,141.2,130.3,130.0,129.2,128.7$, 128.3, 126.7, 125.7, 119.4, 101.9, 42.1, 32.4, 8.4; IR $\widetilde{\boldsymbol{v}}_{\max } 3126(\mathrm{w}), 3059(\mathrm{w}), 3028(\mathrm{w}), 2976(\mathrm{w})$, 2936 (w), 1755 (w), 1687 (m), 1600 (w), 1553 (w), 1492 (m), 1449 (m), 1409 (w), 1343 (w), 1210 (w), 1148 (m), 1070 (m), 1027 (w), 989 (w), 913 (m), 851 (w), 814 (w), 762 (s), 732 (m), 698 (s); HRMS (ESI) calcd for $\mathrm{C}_{18} \mathrm{H}_{17} \mathrm{O}^{+}[\mathrm{M}+\mathrm{H}]^{+} 249.1274$; found 249.1279.

\section{(1-(Trifluoromethyl)cycloprop-2-ene-1,2-diyl)dibenzene (80)}

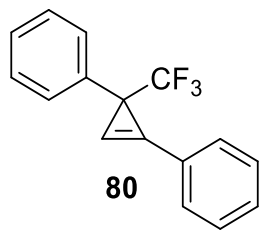

Following the general procedure B, starting from (1-diazo-2,2,2-trifluoroethyl)benzene (56) (1.0 g, $5.4 \mathrm{mmol}, 1.0$ equiv.) and phenylacetylene (1.77 mL, $16.1 \mathrm{mmol}, 3.0$ equiv.), the title compound 80 was obtained after purification by column chromatography on Biotage (Büchi flashpure cartridge $40 \mathrm{~g}$, Pentane) as a yellow oil (867 mg, $3.33 \mathrm{mmol}, 62 \%$ yield). ${ }^{\mathbf{1}} \mathbf{H}$ NMR (400 MHz, Chloroform-d) $\delta 7.69-7.63(\mathrm{~m}, 2 \mathrm{H}, \mathrm{ArH}), 7.50-7.39(\mathrm{~m}, 5 \mathrm{H}, \mathrm{Ar} H), 7.35-7.29(\mathrm{~m}, 2 \mathrm{H}, \mathrm{ArH})$, 7.29 - 7.24 (m, 1H, ArH), 7.17 (q, J = $1.6 \mathrm{~Hz}, 1 \mathrm{H}, \mathrm{C}=\mathrm{CH}) ;{ }^{\mathbf{1 3}} \mathrm{C}$ NMR (101 MHz, Chloroform-d) $138.3,130.5,130.0,128.9,128.4,126.7$ (q, $J=278.4$ Hz), 127.9 (q, $J=1.7 \mathrm{~Hz}), 125.3,117.4$ (q, $J=2.4 \mathrm{~Hz}), 98.9(\mathrm{q}, J=3.3 \mathrm{~Hz}), 32.1(\mathrm{q}, J=35.6 \mathrm{~Hz})$. The characterization data correspond to the reported values. ${ }^{23}$

\footnotetext{
${ }^{23}$ M. Uehara, H. Suematsu, Y. Yasutomi, T. Katsuki, J. Am. Chem. Soc. 2011, 133, 170-171.
} 
Ethyl 1-(4-bromophenyl)-2-phenylcycloprop-2-ene-1-carboxylate (81)

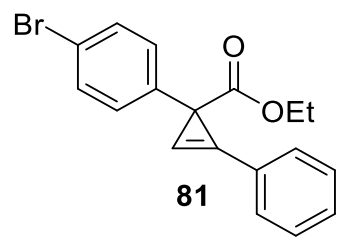

Following the general procedure B, starting from ethyl 2-(4-bromophenyl)-2-diazoacetate (57) (1.32 g, $4.91 \mathrm{mmol}, 1.0$ equiv.) and phenylacetylene (1.62 mL, $14.7 \mathrm{mmol}, 3.0$ equiv.), the title compound 81 was obtained after purification by column chromatography on Biotage (Büchi flashpure cartridge $40 \mathrm{~g}$, Pentane:EtOAc 90:10) as a pale yellow oil (869 mg, $2.53 \mathrm{mmol}, 52 \%$ yield). ${ }^{1} \mathbf{H}$ NMR (400 MHz, Chloroform-d) $\delta 7.60$ - 7.55 (m, 2H, ArH), $7.45-7.35$ (m, 5H, ArH), $7.27-7.22(\mathrm{~m}, 2 \mathrm{H}, \mathrm{ArH}), 7.16(\mathrm{~s}, 1 \mathrm{H}, \mathrm{C}=\mathrm{CH}), 4.18\left(\mathrm{q}, J=7.1 \mathrm{~Hz}, 2 \mathrm{H}, \mathrm{CH}_{2} \mathrm{CH}_{3}\right), 1.20(\mathrm{t}, J=7.1$ $\left.\mathrm{Hz}, 3 \mathrm{H}, \mathrm{CH}_{2} \mathrm{CH}_{3}\right) ;{ }^{13} \mathbf{C}$ NMR (101 MHz, Chloroform-d) $\delta$ 174.2, 140.1, 131.3, 130.3, 130.2, $130.0,129.0,125.3,120.5,117.1,99.8,61.3,33.4,14.4$. The characterization data correspond to the reported values. ${ }^{24}$

Methyl 1-(4-methoxyphenyl)-2-phenylcycloprop-2-ene-1-carboxylate (82)

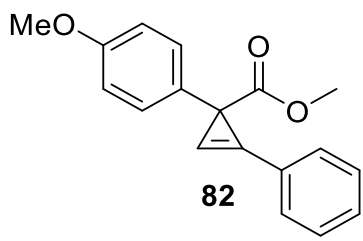

Following the general procedure B, starting from methyl 2-diazo-2-(4-methoxyphenyl)acetate (58) (412 mg, $2.00 \mathrm{mmol}, 1.0$ equiv.) and phenylacetylene (0.66 mL, $6.0 \mathrm{mmol}, 3.0$ equiv.), the title compound 82 was obtained after purification by column chromatography on Biotage (Büchi flashpure cartridge $40 \mathrm{~g}$, Pentane:EtOAc 90:10) as a yellow oil (252 mg, $0.899 \mathrm{mmol}, 45 \%$ yield). ${ }^{1}$ H NMR (400 MHz, Chloroform-d) $\delta 7.65-7.58(\mathrm{~m}, 2 \mathrm{H}, \mathrm{ArH}), 7.46-7.36(\mathrm{~m}, 3 \mathrm{H}, \mathrm{ArH}), 7.34$ - $7.28(\mathrm{~m}, 2 \mathrm{H}, \mathrm{ArH}), 7.21(\mathrm{~s}, 1 \mathrm{H}, \mathrm{C}=\mathrm{CH}), 6.89-6.79(\mathrm{~m}, 2 \mathrm{H}, \mathrm{ArH}), 3.78\left(\mathrm{~s}, 3 \mathrm{H}, \mathrm{OCH}_{3}\right), 3.71(\mathrm{~s}$, $\left.3 \mathrm{H}, \mathrm{CO}_{2} \mathrm{CH}_{3}\right) ;{ }^{13} \mathbf{C}$ NMR (101 MHz, Chloroform-d) $\delta$ 175.5, 158.4, 133.2, 130.1, 130.0, 129.4, $129.0,125.6,117.8,113.7,100.7,55.4,52.4,33.0$. The characterization data correspond to the reported values. $^{25}$

\section{Methyl 2-(4-(tert-butyl)phenyl)-1-(p-tolyl)cycloprop-2-enecarboxylate (83)}

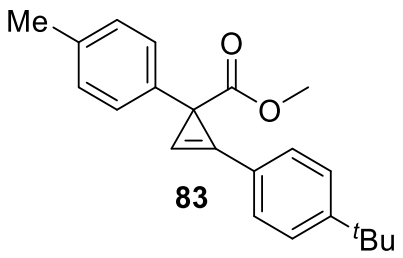

\footnotetext{
${ }^{24}$ R. Hommelsheim, Y. Guo, Z. Yang, C. Empel, R. M. Koenigs, Angew. Chemie Int. Ed. 2019, 58, 1203-1207.

${ }^{25}$ H. M. L. Davies, G. H. Lee, Org. Lett. 2004, 6, 1233-1236.
} 
Following the general procedure B, starting from methyl 2-diazo-2-( $p$-tolyl)acetate (59) (380 mg, $2.00 \mathrm{mmol}, 1.0$ equiv.) and 1-(tert-butyl)-4-ethynylbenzene (1.1 mL, $6.0 \mathrm{mmol}, 3.0$ equiv.), the title compound 83 was obtained after purification by column chromatography on Biotage (Büchi flashpure cartridge $40 \mathrm{~g}$, Pentane:EtOAc 95:5) as a yellow oil (282 mg, $0.880 \mathrm{mmol}, 44 \%$ yield). $\mathbf{R}_{\boldsymbol{f}} 0.48$ (Pentane:EtOAc 90:10); ${ }^{1} \mathbf{H}$ NMR (400 MHz, Chloroform-d) $\delta 7.59-7.53$ (m, 2H, ArH), $7.47-7.43(\mathrm{~m}, 2 \mathrm{H}, \mathrm{ArH}), 7.31-7.26(\mathrm{~m}, 2 \mathrm{H}, \operatorname{ArH}), 7.14(\mathrm{~s}, 1 \mathrm{H}, \mathrm{C}=\mathrm{CH}), 7.12-7.07$ (m, 2H, $\mathrm{ArH}), 3.71\left(\mathrm{~s}, 3 \mathrm{H}, \mathrm{CO}_{2} \mathrm{CH}_{3}\right), 2.31\left(\mathrm{~s}, 3 \mathrm{H}, \mathrm{CH}_{3}\right), 1.33\left(\mathrm{~s}, 9 \mathrm{H},{ }^{t} \mathrm{Bu}\right) ;{ }^{13} \mathrm{C}$ NMR (101 MHz, Chloroform- $d$ ) $\delta 175.5,153.5,138.2,136.2,129.8,128.9,128.3,126.0,122.8,117.4,99.4,52.3$, 35.1, 33.3, 31.3, 21.2; IR $\widetilde{\boldsymbol{v}}_{\max } 3666$ (w), 3134 (w), 2961 (s), 2912 (m), 1910 (w), 1720 (s), 1608 (w), $1508(\mathrm{~m}), 1457(\mathrm{~m}), 1442(\mathrm{~m}), 1402$ (m), $1371(\mathrm{~m}), 1277$ (s), 1212 (s), 1109 (m), 1027 (s), $904(\mathrm{~m}), 833(\mathrm{~s}), 766(\mathrm{~m}), 732(\mathrm{~m}), 689(\mathrm{~m})$; HRMS (ESI) calcd for $\mathrm{C}_{22} \mathrm{H}_{25} \mathrm{O}_{2}{ }^{+}[\mathrm{M}+\mathrm{H}]^{+} 321.1849$; found 321.1843.

Methyl 1-(p-tolyl)-2-(4-(trifluoromethoxy)phenyl)cycloprop-2-enecarboxylate (84)

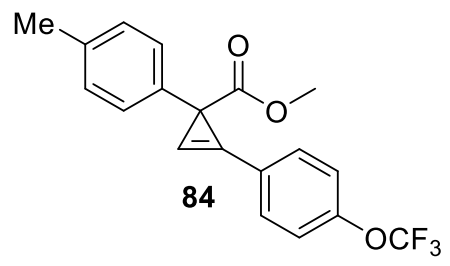

Following the general procedure B, starting from methyl 2-diazo-2-( $p$-tolyl)acetate (59) (380 mg, $2.00 \mathrm{mmol}, 1.0$ equiv.) and 1-ethynyl-4-(trifluoromethoxy)benzene (0.92 mL, $6.0 \mathrm{mmol}, 3.0$ equiv.), the title compound $\mathbf{8 4}$ was obtained after purification by column chromatography on Biotage (Büchi flashpure cartridge $40 \mathrm{~g}$, Pentane:EtOAc 95:5) as a yellow oil (312 mg, 0.896 mmol, $40 \%$ yield, 94\% purity). $\mathbf{R}_{f} 0.45$ (Pentane:EtOAc 90:10); ${ }^{1} \mathbf{H}$ NMR (400 MHz, Chloroform- $d) \delta 7.85-7.77(\mathrm{~m}, 2 \mathrm{H}, \mathrm{ArH}), 7.46-7.41(\mathrm{~m}, 5 \mathrm{H}, \mathrm{ArH} \& \mathrm{C}=\mathrm{CH}), 7.31-7.25(\mathrm{~m}$, 2H, $\mathrm{ArH}$ ), 3.89 (s, 3H, $\left.\mathrm{CO}_{2} \mathrm{CH}_{3}\right), 2.49$ (s, 3H, CH 3$) ;{ }^{13} \mathbf{C}$ NMR (101 MHz, Chloroform-d) $\delta 175.0$, $150.5-150.0(\mathrm{~m}), 137.6,136.5,131.5,129.1,128.1,124.4,121.5,120.4$ (q, $J=278.2 \mathrm{~Hz}), 116.8$, 101.5, 52.0, 33.6, 21.2; ${ }^{19}$ F NMR (376 MHz, Chloroform-d) $\delta$-57.7; IR $\widetilde{\boldsymbol{v}}_{\max } 3133(\mathrm{w}), 2995(\mathrm{w})$, 2952 (w), 1721 (m), 1597 (w), 1506 (m), 1441 (w), 1255 (s), 1212 (s), 1169 (s), 1113 (m), 1024 (m), $906(\mathrm{~m}), 848(\mathrm{~m}), 819(\mathrm{~m}), 766(\mathrm{w}), 731(\mathrm{~m}), 692(\mathrm{w})$; HRMS (ESI) calcd for $\mathrm{C}_{19} \mathrm{H}_{16} \mathrm{~F}_{3} \mathrm{O}_{3}{ }^{+}$ $[\mathrm{M}+\mathrm{H}]^{+} 349.1046$; found 349.1052.

Methyl 2-(2-chlorophenyl)-1-phenylcycloprop-2-ene-1-carboxylate (85)

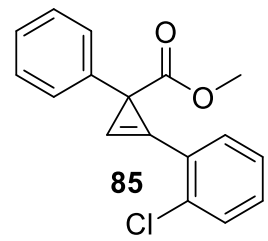

Following the general procedure B, starting from methyl 2-diazo-2-phenylacetate (49) (352 mg, $2.00 \mathrm{mmol}, 1.0$ equiv.) and 1-chloro-2-ethynylbenzene $(0.73 \mathrm{~mL}, 6.0 \mathrm{mmol}, 3.0$ equiv.), the title compound 85 was obtained after purification by column chromatography on Biotage (Büchi flashpure cartridge $40 \mathrm{~g}$, Pentane:EtOAc 95:5) as a yellow oil (329 mg, $1.16 \mathrm{mmol}, 58 \%$ yield). 
${ }^{1}$ H NMR (400 MHz, Chloroform- $d$ ) $\delta 7.56-7.53(\mathrm{~m}, 1 \mathrm{H}, \operatorname{Ar} H), 7.51-7.47(\mathrm{~m}, 1 \mathrm{H}, \operatorname{Ar} H), 7.45$ (s, 1H, C=CH), $7.41-7.36(\mathrm{~m}, 2 \mathrm{H}, \operatorname{Ar} H), 7.35-7.27(\mathrm{~m}, 4 \mathrm{H}, \mathrm{Ar} H), 7.25-7.19(\mathrm{~m}, 1 \mathrm{H}, \operatorname{Ar} H)$, 3.73 (s, $\left.3 \mathrm{H}, \mathrm{CO}_{2} \mathrm{CH}_{3}\right) ;{ }^{13} \mathbf{C}$ NMR (101 MHz, Chloroform-d) $\delta$ 174.8, 140.6, 136.4, 131.7, 131.0, $130.2,128.3,128.3,127.1,126.8,124.4,115.1,105.1,52.4,33.4$. The characterization data correspond to the reported values. ${ }^{26}$

\section{Methyl 1-(2-fluorophenyl)-2-phenylcycloprop-2-ene-1-carboxylate (86)}

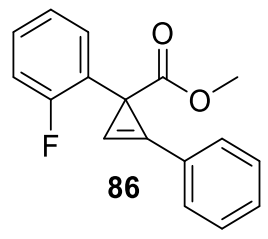

Following the general procedure B, starting from methyl 2-diazo-2-(2-fluorophenyl)acetate (60) (900 mg, $4.64 \mathrm{mmol}, 1.0$ equiv.) and phenylacetylene (1.53 mL, $13.9 \mathrm{mmol}, 3.0$ equiv.), the title compound 86 was obtained after purification by column chromatography on Biotage (Büchi flashpure cartridge $40 \mathrm{~g}$, Pentane:EtOAc 90:10) as a pale yellow oil (734 mg, $2.74 \mathrm{mmol}, 59 \%$ yield). ${ }^{1} \mathrm{H}$ NMR (400 MHz, Chloroform- $\boldsymbol{d}$ ) $\delta 7.73-7.66(\mathrm{~m}, 2 \mathrm{H}, \mathrm{Ar} H), 7.48-7.38(\mathrm{~m}, 3 \mathrm{H}, \mathrm{Ar} H$ ), $7.27(\mathrm{~s}, 1 \mathrm{H}, \mathrm{C}=\mathrm{CH}), 7.25-7.16(\mathrm{~m}, 2 \mathrm{H}, \mathrm{Ar} H), 7.09-7.05(\mathrm{~m}, 1 \mathrm{H}, \mathrm{ArH}), 7.05-7.01(\mathrm{~m}, 1 \mathrm{H}$, $\mathrm{Ar} H), 3.71\left(\mathrm{~s}, 3 \mathrm{H}, \mathrm{CO}_{2} \mathrm{CH}_{3}\right) ;{ }^{13} \mathbf{C}$ NMR (101 MHz, Chloroform- $\left.d\right) \delta 174.9,161.7(\mathrm{~d}, J=244.6$ $\mathrm{Hz}), 130.2,130.1,130.0(\mathrm{~d}, J=4.5 \mathrm{~Hz}), 129.2,128.9(\mathrm{~d}, J=8.7 \mathrm{~Hz}), 125.4,124.2(\mathrm{~d}, J=3.4 \mathrm{~Hz})$, $119.2,115.5(\mathrm{~d}, J=22.1 \mathrm{~Hz}), 99.7,52.3,29.8$. The characterization data correspond to the reported values. $^{24}$

\section{Methyl 1-(2-chloro-4-fluorophenyl)-2-(p-tolyl)cycloprop-2-enecarboxylate (87)}

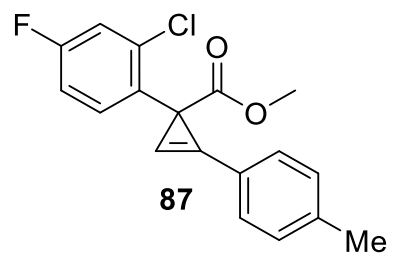

Following the general procedure $\mathrm{B}$, starting from methyl 2-(2-chloro-4-fluorophenyl)-2diazoacetate (61) (941 mg, $4.12 \mathrm{mmol}, 1.0$ equiv.) and 1-ethynyl-4-methylbenzene (1.60 mL, 12.4 mmol, 3.0 equiv.), the title compound $\mathbf{8 7}$ was obtained after purification by column chromatography on Biotage (Büchi flashpure cartridge $40 \mathrm{~g}$, Pentane:EtOAc 95:5) as an orange oil (439 mg, $1.39 \mathrm{mmol}, 32 \%$ yield, 95\% purity). $\mathbf{R}_{f} 0.32$ (Pentane:EtOAc 90:10); ${ }^{1} \mathbf{H}$ NMR (400 MHz, Chloroform- $d$ ) $\delta 7.52(\mathrm{~d}, J=8.1 \mathrm{~Hz}, 2 \mathrm{H}, \operatorname{Ar} H), 7.21-7.17(\mathrm{~m}, 3 \mathrm{H}, \operatorname{Ar} H), 7.16(\mathrm{~s}, 1 \mathrm{H}$, $\mathrm{C}=\mathrm{CH}) 7.02(\mathrm{dd}, J=8.5,2.6 \mathrm{~Hz}, 1 \mathrm{H}, \mathrm{Ar} H), 6.77$ (ddd, $J=8.5,8.0,2.6 \mathrm{~Hz}, 1 \mathrm{H}, \mathrm{Ar} H), 3.63$ (s, 3H, $\left.\mathrm{CO}_{2} \mathrm{CH}_{3}\right), 2.32$ (s, 3H, $\left.\mathrm{CH}_{3}\right) ;{ }^{13} \mathrm{C}$ NMR (101 MHz, Chloroform-d) $\delta 174.6,161.6(\mathrm{~d}, J=248.9$ $\mathrm{Hz}), 140.7,136.2(\mathrm{~d}, J=3.7 \mathrm{~Hz}), 135.7(\mathrm{~d}, J=10.4 \mathrm{~Hz}), 130.9(\mathrm{~d}, J=8.8 \mathrm{~Hz}), 129.9,129.7,122.6$, $118.1,116.9(\mathrm{~d}, J=24.8 \mathrm{~Hz}), 114.0(\mathrm{~d}, J=21.0 \mathrm{~Hz}), 100.6,52.5,32.7,21.6 ;{ }^{19} \mathbf{F}$ NMR (376 MHz, Chloroform- $d$ ) $\delta-113.4\left(\mathrm{td}, J=8.2,5.9 \mathrm{~Hz}\right.$ ); IR $\widetilde{\boldsymbol{v}}_{\max } 3139(\mathrm{w}), 3028(\mathrm{w}), 2951(\mathrm{w}), 1723(\mathrm{~s})$, 1597 (m), 1490 (m), 1442 (m), 1386 (w), 1224 (s), 1115 (w), 1049 (m), 1012 (m), 912 (m), 862

${ }^{26}$ L. Chen, D. Leslie, M. G. Coleman, J. Mack, Chem. Sci. 2018, 9, 4650-4661. 
(m), 821 (m), $776(\mathrm{~m}), 730$ (s), 687 (m), 654 (w), 604 (w) ; HRMS (ESI) calcd for $\mathrm{C}_{18} \mathrm{H}_{15} \mathrm{ClFO}_{2}{ }^{+}$ $[\mathrm{M}+\mathrm{H}]^{+}$317.0739; found 317.0745.

Methyl 2-(3,5-bis(trifluoromethyl)phenyl)-1-phenylcycloprop-2-ene-1-carboxylate (88)

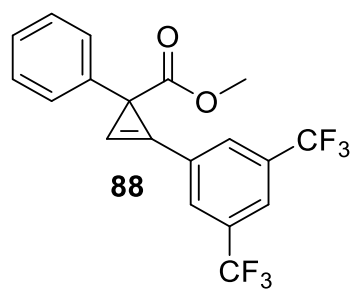

Following the general procedure B, starting from methyl 2-diazo-2-phenylacetate (49) (352 mg, $2.00 \mathrm{mmol}, 1.0$ equiv.) and 1-ethynyl-3,5-bis(trifluoromethyl)benzene (1.1 mL, $6.0 \mathrm{mmol}, 3.0$ equiv.), the title compound $\mathbf{8 8}$ was obtained after purification by column chromatography on Biotage (Büchi flashpure cartridge $40 \mathrm{~g}$, Pentane:EtOAc 97:3) as a yellow oil (333 $\mathrm{mg}, 0.862$ mmol, $43 \%$ yield). $\mathbf{R}_{\boldsymbol{f}} 0.30$ (Pentane:EtOAc 95:5); ${ }^{1} \mathrm{H}$ NMR (400 MHz, Chloroform- $d$ ) $\delta 8.00$ (s, 2H, $\operatorname{ArH}), 7.86(\mathrm{~s}, 1 \mathrm{H}, \operatorname{ArH}), 7.50(\mathrm{~s}, 1 \mathrm{H}, \mathrm{C}=\mathrm{CH}), 7.34-7.31(\mathrm{~m}, 2 \mathrm{H}, \mathrm{ArH}), 7.31-7.28(\mathrm{~m}, 2 \mathrm{H}$, $\mathrm{ArH}), 7.26-7.23(\mathrm{~m}, 1 \mathrm{H}, \mathrm{ArH}), 3.73\left(\mathrm{~s}, 3 \mathrm{H}, \mathrm{CO}_{2} \mathrm{CH}_{3}\right) ;{ }^{13} \mathrm{C}$ NMR (101 MHz, Chloroform-d) $\delta$ 174.2, 139.7, $132.7(\mathrm{q}, J=33.8 \mathrm{~Hz}), 129.7-129.4(\mathrm{~m}), 128.6,128.1,128.0,127.3,123.5(\mathrm{p}, J=$ $3.8 \mathrm{~Hz}), 123.0$ (q, $J=272.9 \mathrm{~Hz}), 116.4,105.2,52.7,34.5 ;{ }^{19}$ F NMR (376 MHz, Chloroform-d) $\delta$ -63.1; IR $\widetilde{\boldsymbol{v}}_{\max } 3136(\mathrm{w}), 3031$ (w), 2956 (w), 2851 (w), 1725 (m), 1610 (w), 1495 (w), 1443 (w), 1373 (m), 1278 (s), 1176 (s), 1131 (s), 1013 (m), 904 (m), 841 (m), 704 (m), 692 (m); HRMS (ESI) calcd for $\mathrm{C}_{19} \mathrm{H}_{12} \mathrm{~F}_{6} \mathrm{O}_{2}{ }^{+}[\mathrm{M}]^{+}$386.0736; found 386.0743.

\section{Methyl 2-phenyl-1-(thiophen-3-yl)cycloprop-2-enecarboxylate (89)}

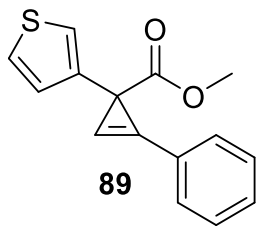

Following the general procedure B, starting from methyl 2-diazo-2-(thiophen-3-yl)acetate (62) (758 mg, $4.16 \mathrm{mmol}, 1.0$ equiv.) and phenylacetylene (1.37 mL, $12.5 \mathrm{mmol}, 3.0$ equiv.), the title compound 89 was obtained after purification by column chromatography on Biotage (Büchi flashpure cartridge $40 \mathrm{~g}$, Pentane:EtOAc 96:4) as a brown oil (490 mg, $1.91 \mathrm{mmol}, 46 \%$ yield). ${ }^{1}$ H NMR (400 MHz, Chloroform-d) $\delta 7.61-7.55$ (m, 2H, ArH), $7.46-7.39(\mathrm{~m}, 3 \mathrm{H}, \mathrm{ArH}), 7.35$ $(\mathrm{dd}, J=3.0,1.3 \mathrm{~Hz}, 1 \mathrm{H}$, HetArH), $7.19(\mathrm{dd}, J=5.0,3.0 \mathrm{~Hz}, 1 \mathrm{H}, \operatorname{HetAr} H), 7.11(\mathrm{~s}, 1 \mathrm{H}, \mathrm{C}=\mathrm{C} H)$, $7.05\left(\mathrm{dd}, J=5.0,1.4 \mathrm{~Hz}, 1 \mathrm{H}\right.$, HetArH), $3.72\left(\mathrm{~s}, 3 \mathrm{H}, \mathrm{CO}_{2} \mathrm{CH}_{3}\right) ;{ }^{13} \mathbf{C}$ NMR (101 MHz, Chloroformd) $\delta 174.7,142.1,130.0,129.9,128.7,127.7,125.0,124.5,121.6,115.6,99.9,52.2,33.0$. The characterization data correspond to the reported values. ${ }^{25}$ 
Methyl 2-(naphthalen-2-yl)-1-phenylcycloprop-2-ene-1-carboxylate (90)

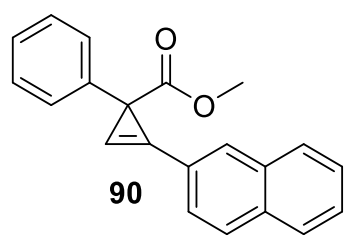

Following the general procedure B, starting from methyl 2-diazo-2-phenylacetate (49) (352 mg, $2.00 \mathrm{mmol}, 1.0$ equiv.) and 2-ethynylnaphthalene (913 $\mathrm{mg}, 6.00 \mathrm{mmol}, 3.0$ equiv.), the title compound 90 was obtained after purification by column chromatography on Biotage (Büchi flashpure cartridge $40 \mathrm{~g}$, Pentane:EtOAc 95:5) as a white solid (389 mg, $1.29 \mathrm{mmol}, 65 \%$ yield). ${ }^{1}$ H NMR (400 MHz, Chloroform-d) $\delta 8.09-8.06$ (m, 1H, ArH), $7.91-7.82(\mathrm{~m}, 3 \mathrm{H}, \mathrm{ArH}), 7.72$ (dd, $J=8.4,1.6 \mathrm{~Hz}, 1 \mathrm{H}, \operatorname{ArH}), 7.56-7.49$ (m, 2H, ArH), $7.48-7.42$ (m, 2H, ArH), $7.33-7.27$ $(\mathrm{m}, 3 \mathrm{H}, \mathrm{ArH} \& \mathrm{C}=\mathrm{CH}), 7.25-7.20(\mathrm{~m}, 1 \mathrm{H}, \mathrm{ArH}), 3.74\left(\mathrm{~s}, 3 \mathrm{H}, \mathrm{CO}_{2} \mathrm{CH}_{3}\right) ;{ }^{\mathbf{1 3}} \mathbf{C} \mathbf{N M R}(\mathbf{1 0 1} \mathbf{M H z}$, Chloroform- $d$ ) $\delta 175.2,141.0,134.0,133.3,130.1,128.9,128.6,128.4,128.3,128.0,127.5,126.9$, $126.7,126.7,122.9,117.2,101.3,52.4,33.9$. The characterization data correspond to the reported values. ${ }^{25}$

\section{Methyl 1-(4-methoxyphenyl)-2-(naphthalen-2-yl)cycloprop-2-enecarboxylate (91)}

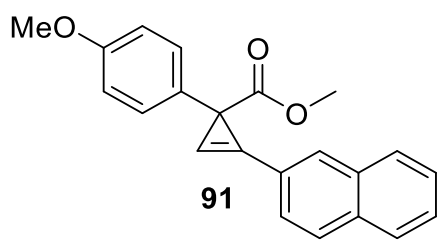

Following the general procedure B, starting from methyl 2-diazo-2-(4-methoxyphenyl)acetate (58) (412 mg, $2.00 \mathrm{mmol}, 1.0$ equiv.) and 2-ethynylnaphthalene (913 mg, $6.00 \mathrm{mmol}, 3.0$ equiv.), the title compound 91 was obtained after purification by column chromatography on Biotage (Büchi flashpure cartridge $40 \mathrm{~g}$, Pentane:EtOAc 90:10) as a yellow oil (220 mg, $0.666 \mathrm{mmol}, 29 \%$ yield, 88\% purity). $\mathbf{R}_{\boldsymbol{f}} 0.32$ (Pentane:EtOAc 90:10); ${ }^{\mathbf{1}} \mathbf{H}$ NMR (400 MHz, Chloroform-d) $\delta 8.10-8.05$ $(\mathrm{m}, 1 \mathrm{H}, \mathrm{ArH}), 7.91-7.82(\mathrm{~m}, 3 \mathrm{H}, \mathrm{ArH}), 7.72(\mathrm{dd}, J=8.5,1.6 \mathrm{~Hz}, 1 \mathrm{H}, \mathrm{ArH}), 7.55-7.50(\mathrm{~m}, 2 \mathrm{H}$, $\operatorname{ArH}), 7.42-7.35(\mathrm{~m}, 2 \mathrm{H}, \operatorname{ArH}), 7.31(\mathrm{~s}, 1 \mathrm{H}, \mathrm{C}=\mathrm{CH}), 6.88-6.82(\mathrm{~m}, 2 \mathrm{H}, \mathrm{ArH}), 3.78(\mathrm{~s}, 3 \mathrm{H}$, $\left.\mathrm{CO}_{2} \mathrm{CH}_{3}\right), 3.74\left(\mathrm{~s}, 3 \mathrm{H}, \mathrm{OCH}_{3}\right) ;{ }^{13} \mathbf{C}$ NMR (101 MHz, Chloroform-d) $\delta$ 175.4, 158.3, 133.9, 133.2, $133.1,129.9,129.4,128.8,128.5,127.9,127.4,126.8,126.6,122.9,117.5,113.6,101.5,55.3$, 52.3, 33.1; IR $\widetilde{\boldsymbol{v}}_{\max } 3134(\mathrm{~m}), 3051(\mathrm{~m}), 3003(\mathrm{~m}), 2952(\mathrm{~m}), 2843(\mathrm{~m}), 2359(\mathrm{~m}), 2333(\mathrm{~m}), 1718$ (s), 1604 (s), 1511 (s), 1460 (s), 1341 (m), 1242 (s), 1218 (s), 1173 (s), 1103 (m), 1029 (s), 904 (s), 859 (s), 822 (s), 738 (s), 653 (s); HRMS (ESI) calcd for $\mathrm{C}_{22} \mathrm{H}_{18} \mathrm{NaO}_{3}{ }^{+}[\mathrm{M}+\mathrm{Na}]^{+} 353.1148$; found 353.1150 . 
Ethyl 2-(4-bromophenyl)-1-(naphthalen-2-yl)cycloprop-2-enecarboxylate (92)

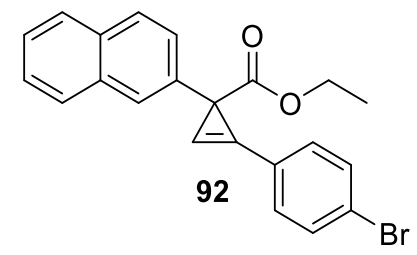

Following the general procedure B, starting from ethyl 2-diazo-2-(naphthalen-2-yl)acetate (63) (481 mg, $2.00 \mathrm{mmol}, 1.0$ equiv.) and 1-bromo-4-ethynylbenzene (1.09 g, $6.00 \mathrm{mmol}, 3.0$ equiv.), the title compound $\mathbf{9 2}$ was obtained after purification by column chromatography on Biotage (Büchi flashpure cartridge $40 \mathrm{~g}$, Pentane:EtOAc 90:10) as a fluffy white solid (543 mg, $1.38 \mathrm{mmol}$, $69 \%$ yield). Rf 0.28 (Pentane:EtOAc 90:10); m.p = 129-130 ${ }^{\circ} \mathrm{C} ;{ }^{1} \mathbf{H}$ NMR (400 MHz, Chloroform-d) $\delta 7.81-7.73(\mathrm{~m}, 4 \mathrm{H}, \mathrm{ArH}), 7.59-7.55(\mathrm{~m}, 2 \mathrm{H}, \mathrm{ArH}), 7.54-7.48(\mathrm{~m}, 3 \mathrm{H}, \mathrm{ArH})$, $7.45-7.38(\mathrm{~m}, 2 \mathrm{H}, \mathrm{ArH}), 7.33(\mathrm{~s}, 1 \mathrm{H}, \mathrm{C}=\mathrm{CH}), 4.23\left(\mathrm{q}, J=7.1 \mathrm{~Hz}, 2 \mathrm{H}, \mathrm{CO}_{2} \mathrm{CH}_{2} \mathrm{CH}_{3}\right), 1.23(\mathrm{t}, J=$ $\left.7.1 \mathrm{~Hz}, 3 \mathrm{H}, \mathrm{CO}_{2} \mathrm{CH}_{2} \mathrm{CH}_{3}\right) ;{ }^{13} \mathbf{C}$ NMR (101 MHz, Chloroform-d) $\delta$ 174.2, 138.3, 133.3, 132.3, 132.2, 131.3, 127.8, 127.6, 127.6, 126.6, 126.5, 126.0, 125.6, 124.5, 124.4, 116.8, 101.3, 61.1, 34.0, 14.3; IR $\widetilde{\boldsymbol{v}}_{\max } 3135(\mathrm{w}), 3056(\mathrm{w}), 2983(\mathrm{~m}), 1715(\mathrm{~s}), 1593(\mathrm{~m}), 1477(\mathrm{~m}), 1392(\mathrm{~m}), 1360$ (m), 1242 (s), 1213 (s), 1176 (s), $1104(\mathrm{~m}), 1075$ (m), 1033 (s), 909 (m), 825 (s), 745 (s), 681 (m); HRMS (ESI) calcd for $\mathrm{C}_{22} \mathrm{H}_{17}{ }^{79} \mathrm{BrNaO}_{2}{ }^{+}[\mathrm{M}+\mathrm{Na}]^{+}$415.0304; found 415.0315.

\section{Methyl 2-(phenanthren-9-yl)-1-phenylcycloprop-2-enecarboxylate (93)}

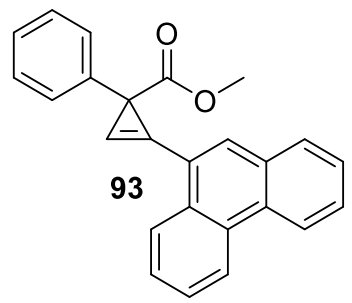

Following the general procedure B, starting from methyl 2-diazo-2-phenylacetate (49) (352 mg, $2.00 \mathrm{mmol}, 1.0$ equiv.) and 9-ethynylphenanthrene (1.21 g, $6.00 \mathrm{mmol}, 3.0$ equiv.), the title compound 93 was obtained after purification by column chromatography on Biotage (Büchi flashpure cartridge $40 \mathrm{~g}$, Pentane:EtOAc 95:5) as a yellow powder (342 mg, $0.976 \mathrm{mmol}, 49 \%$ yield). $\mathbf{R}_{\boldsymbol{f}} 0.35$ (Pentane:EtOAc 90:10); m.p = 150-151 ${ }^{\circ} \mathrm{C} ; \mathbf{1}^{\mathbf{1}} \mathbf{H}$ NMR (400 MHz, Chloroform- $\boldsymbol{d}$ ) $\delta 8.78-8.71(\mathrm{~m}, 1 \mathrm{H}, \operatorname{Ar} H), 8.68(\mathrm{~d}, J=8.3 \mathrm{~Hz}, 1 \mathrm{H}, \operatorname{Ar} H), 8.57-8.48(\mathrm{~m}, 1 \mathrm{H}, \operatorname{ArH}), 8.01(\mathrm{~s}, 1 \mathrm{H}$, $\operatorname{ArH}), 7.91(\mathrm{dd}, J=7.9,1.4 \mathrm{~Hz}, 1 \mathrm{H}, \operatorname{ArH}), 7.76-7.68(\mathrm{~m}, 3 \mathrm{H}, \operatorname{ArH}), 7.64-7.59$ (m, 2H, ArH \& $\mathrm{C}=\mathrm{CH}), 7.55-7.50(\mathrm{~m}, 2 \mathrm{H}, \operatorname{ArH}), 7.35-7.28(\mathrm{~m}, 2 \mathrm{H}, \operatorname{ArH}), 7.25-7.20(\mathrm{~m}, 1 \mathrm{H}, \operatorname{Ar} H), 3.76(\mathrm{~s}$, $\left.3 \mathrm{H}, \mathrm{CO}_{2} \mathrm{CH}_{3}\right) ;{ }^{13} \mathbf{C} \mathbf{N M R}(\mathbf{1 0 1} \mathbf{M H z}$, Chloroform-d) $\delta$ 175.1, 140.8, 132.0, 131.3, 131.2, 130.8, $130.6,129.7,128.5,128.4,128.3,127.5,127.4,127.3,126.7,125.4,123.2,122.8,120.5,115.1$, 103.9, 52.4, 32.1; IR $\widetilde{\boldsymbol{v}}_{\max } 3130$ (w), 3060 (w), 3027 (w), 2951 (w), 2253 (w), 1717 (s), $1601(\mathrm{w})$, 1495 (m), 1442 (m), 1283 (m), 1217 (s), $1114(\mathrm{~m}), 1027$ (m), 907 (s), 857 (m), 733 (s), 613 (m); HRMS (ESI) calcd for $\mathrm{C}_{25} \mathrm{H}_{19} \mathrm{O}_{2}{ }^{+}[\mathrm{M}+\mathrm{H}]^{+} 351.1380$; found 351.1385 . 


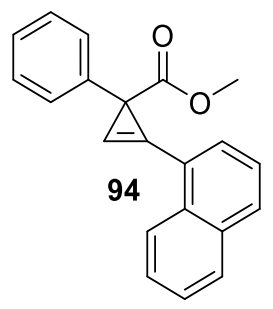

Following the general procedure B, starting from methyl 2-diazo-2-phenylacetate (49) (640 mg, $3.63 \mathrm{mmol}, 1.0$ equiv.) and 1-ethynylnaphthalene (1.55 mL, $10.9 \mathrm{mmol}, 3.0$ equiv.), the title compound 94 was obtained after purification by column chromatography on Biotage (Büchi flashpure cartridge $40 \mathrm{~g}$, Pentane:EtOAc 95:5) as a yellow oil (556 mg, $1.85 \mathrm{mmol}, 51 \%$ yield). ${ }^{1}$ H NMR (400 MHz, Chloroform-d) $\delta 8.45(\mathrm{~d}, J=8.1 \mathrm{~Hz}, 1 \mathrm{H}, \operatorname{Ar} H), 7.92(\mathrm{dd}, J=7.8,3.0 \mathrm{~Hz}$, 2H, ArH), 7.75 (dd, J=7.1, 1.2 Hz, 1H, ArH), $7.71-7.63$ (m, 1H, ArH), $7.62-7.48$ (m, 5H, ArH), $7.34-7.28(\mathrm{~m}, 2 \mathrm{H}, \mathrm{ArH}), 7.28-7.21(\mathrm{~m}, 1 \mathrm{H}, \mathrm{ArH}), 3.76\left(\mathrm{~s}, 3 \mathrm{H}, \mathrm{CO}_{2} \mathrm{CH}_{3}\right) ;{ }^{13} \mathbf{C}$ NMR (101 MHz, Chloroform- $d$ ) $\delta 175.1,140.9,133.7,132.4,130.9,130.0,128.8,128.7,128.4,128.2,127.5,126.7$, $126.6,124.4,121.8,115.4,102.9,52.4,32.1$. The characterization data correspond to the reported values. ${ }^{27}$

Methyl 1,2-di(naphthalen-1-yl)cycloprop-2-enecarboxylate (95)

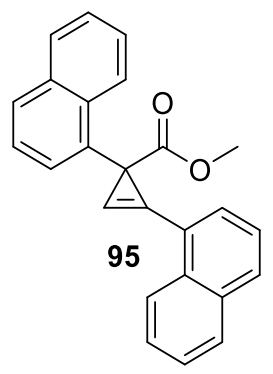

Following the general procedure B, starting from methyl 2-diazo-2-(naphthalen-1-yl)acetate (64) (588 mg, $2.60 \mathrm{mmol}, 1.0$ equiv.) and 1-ethynylnaphthalene (1.1 mL, $7.8 \mathrm{mmol}, 3.0$ equiv.), the title compound 95 was obtained after purification by column chromatography on Biotage (Büchi flashpure cartridge $40 \mathrm{~g}$, Pentane:EtOAc 90:10) as an orange powder (143 mg, $0.408 \mathrm{mmol}, 14 \%$ yield, 90\% purity). $\mathbf{R}_{\boldsymbol{f}} 0.29$ (Pentane:EtOAc 90:10); m.p $=172-173{ }^{\circ} \mathrm{C} ;{ }^{\mathbf{1}} \mathbf{H}$ NMR (400 $\mathbf{~ M H z}$, Chloroform- $d$ ) $\delta 8.42-8.35(\mathrm{~m}, 1 \mathrm{H}, \operatorname{Ar} H), 8.22(\mathrm{dt}, J=8.4,1.0 \mathrm{~Hz}, 1 \mathrm{H}, \operatorname{ArH}), 8.01-7.87(\mathrm{~m}$, $4 \mathrm{H}, \operatorname{Ar} H), 7.84(\mathrm{~s}, 1 \mathrm{H}, \mathrm{C}=\mathrm{CH}), 7.76(\mathrm{dt}, J=8.2,1.2 \mathrm{~Hz}, 1 \mathrm{H}, \mathrm{ArH}), 7.62-7.55(\mathrm{~m}, 5 \mathrm{H}, \mathrm{ArH}), 7.54$ $-7.50(\mathrm{~m}, 1 \mathrm{H}, \mathrm{ArH}), 7.31(\mathrm{dd}, J=8.2,7.1 \mathrm{~Hz}, 1 \mathrm{H}, \mathrm{ArH}), 3.70\left(\mathrm{~s}, 3 \mathrm{H}, \mathrm{CO}_{2} \mathrm{CH}_{3}\right) ;{ }^{13} \mathbf{C}$ NMR (101 MHz, Chloroform- $d$ ) $\delta$ 176.1 139.0, 134.0, 133.8, 132.6, 132.5, 131.0, 129.6, 129.0, 128.8, 128.0, 127.5, 126.8, 126.3 125.9, 125.8, 125.8, 125.6, 124.8, 124.6, 122.7, 116.4, 106.6, 52.7, 31.0; IR $\widetilde{\boldsymbol{v}}_{\max } 3132(\mathrm{w}), 3054(\mathrm{~m}), 2949(\mathrm{w}), 1939(\mathrm{w}), 1714(\mathrm{~s}), 1589(\mathrm{~m}), 1504(\mathrm{~m}), 1439(\mathrm{~m}), 1397(\mathrm{~m})$, $1288(\mathrm{~m}), 1226$ (s), $1174(\mathrm{~m}), 1084$ (m), 1053 (m), 1011 (m), 994 (m), 910 (s), 778 (s), 731 (s), 647 (m) ; HRMS (ESI) calcd for $\mathrm{C}_{25} \mathrm{H}_{19} \mathrm{O}_{2}{ }^{+}[\mathrm{M}+\mathrm{H}]^{+} 351.1380$; found 351.1380.

${ }^{27}$ C. Li, Y. Zeng, J. Wang, Tetrahedron Lett. 2009, 50, 2956-2959. 


\section{Synthesis of the ABZ Reagent 3}

\section{2-Iodo- $N$-tosylbenzamide (97)}

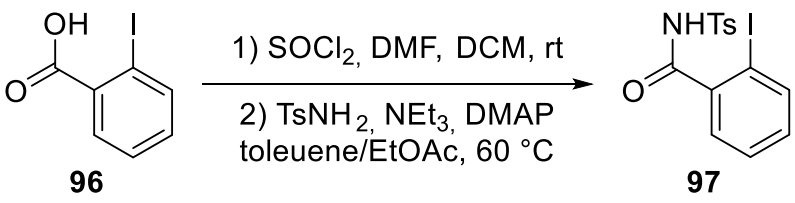

Following a reported procedure, ${ }^{28} \mathrm{SOCl}_{2}(3.53 \mathrm{~mL}, 48.4 \mathrm{mmol}, 1.2$ equiv) followed by DMF $(0.20$ $\mathrm{mL}, 2.6 \mathrm{mmol}, 0.06$ equiv) were added dropwise to a solution of 2-iodobenzoic acid (96) (10.0 g, $40.3 \mathrm{mmol}, 1.0$ equiv.) in DCM $(200 \mathrm{~mL}, 0.2 \mathrm{M})$, and the resulting mixture was stirred for $3 \mathrm{~h} \mathrm{at}$ room temperature. Thereafter, the solvent was evaporated, the residue was dissolved in toluene (32 $\mathrm{mL})$, and then the mixture was added to a solution of 4-toluenesulfoneamide (6.90 g, $40.3 \mathrm{mmol}$, 1.0 equiv.), triethylamine (14.0 mL, $101 \mathrm{mmol}, 2.5$ equiv), and DMAP (250 mg, $2.02 \mathrm{mmol}, 0.05$ equiv.) in EtOAc $(80 \mathrm{~mL})$. The mixture was heated for $1 \mathrm{~h}$ to $60{ }^{\circ} \mathrm{C}$, then cooled to room temperature, and quenched with $1 \mathrm{M} \mathrm{HCl}(50 \mathrm{~mL})$. The mixture was extracted with EtOAc $(3 \times 50$ $\mathrm{mL}$ ), and the combined organic extracts were washed with brine, dried over $\mathrm{MgSO}_{4}$, filtered, and concentrated under reduced pressure. The crude residue was purified by column chromatography $\left(\mathrm{SiO}_{2}, \mathrm{DCM}\right)$ affording $97\left(15.8 \mathrm{~g}, 39.5 \mathrm{mmol}, 98 \%\right.$ yield) as a colorless oil. ${ }^{\mathbf{1}} \mathbf{H}$ NMR (400 MHz, Chloroform-d) $\delta 8.78(\mathrm{~s}, 1 \mathrm{H}, \mathrm{NH}), 8.01(\mathrm{~d}, J=8.4 \mathrm{~Hz}, 2 \mathrm{H}, \operatorname{Ar} H), 7.80(\mathrm{dd}, J=8.0,1.0 \mathrm{~Hz}, 1 \mathrm{H}$, $\mathrm{ArH}), 7.43-7.31(\mathrm{~m}, 4 \mathrm{H}, \mathrm{ArH}), 7.10$ (ddd, $J=8.0,7.2,2.0 \mathrm{~Hz}, 1 \mathrm{H}, \mathrm{ArH}), 2.44\left(\mathrm{~s}, 3 \mathrm{H}, \mathrm{CH}_{3}\right) ;{ }^{13} \mathbf{C}$ NMR (101 MHz, Chloroform-d) $\delta$ 165.6, 145.3, 140.2, 138.4, 134.9, 132.4, 129.5, 128.8, 128.7, $128.3,91.6,21.7$. The characterization data is corresponding to the reported values. ${ }^{28}$

\section{3-Oxo-2-tosyl-2,3-dihydro-1H-1 $\lambda^{3}$-benzo[d][1,2]iodazol-1-yl acetate (98)}

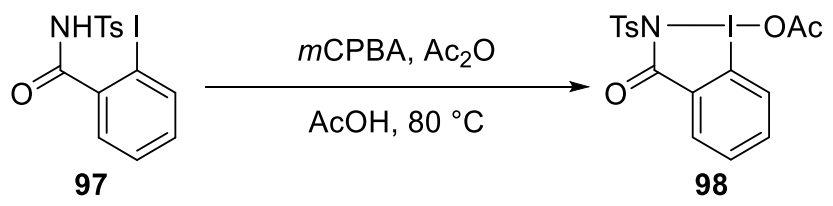

Caution: For safety reasons, the reaction was carried out behind an antiblast shield. Following a reported procedure, ${ }^{28} \mathrm{mCPBA}$ (77\% pure; $8.85 \mathrm{~g}, 39.6 \mathrm{mmol}, 1.0$ equiv.) was added to a solution of 97 (15.8 g, $39.5 \mathrm{mmol}, 1.0$ equiv.) and $\mathrm{Ac}_{2} \mathrm{O}$ (160 mL, $1.68 \mathrm{~mol}, 42.4$ equiv.) in $\mathrm{AcOH}$ (158 $\mathrm{mL}, 0.10 \mathrm{M}$ ), and the resulting mixture was heated for $48 \mathrm{~h}$ to $80{ }^{\circ} \mathrm{C}$. Thereafter, the mixture was cooled to room temperature, and diethyl ether $(150 \mathrm{~mL})$ was added. Compound $98(7.13 \mathrm{~g}, 15.5$ mmol, $39 \%$ yield) crystallized at $0{ }^{\circ} \mathrm{C}$ from the solution and was collected by filtration. ${ }^{1} \mathbf{H}$ NMR (400 MHz, Chloroform-d) $\delta 8.02-7.95(\mathrm{~m}, 2 \mathrm{H}, \mathrm{ArH}), 7.95-7.89(\mathrm{~m}, 2 \mathrm{H}, \mathrm{ArH}), 7.86(\mathrm{dd}, J=$ 8.8, $0.9 \mathrm{~Hz}, 1 \mathrm{H}, \mathrm{ArH}), 7.80-7.71(\mathrm{~m}, 1 \mathrm{H}, \mathrm{ArH}), 7.44(\mathrm{~d}, J=8.1 \mathrm{~Hz}, 2 \mathrm{H}, \mathrm{ArH}), 2.38\left(\mathrm{~s}, 3 \mathrm{H}, \mathrm{CH}_{3}\right)$, 2.26 (s, 3H, $\left.\mathrm{OCOCH}_{3}\right) ;{ }^{13} \mathbf{C}$ NMR (101 MHz, Chloroform-d) $\delta 174.1,162.4,144.5,136.8,136.0$, $132.3,131.4,131.2,129.7,128.8,127.9,118.0,21.1,20.2$. The characterization data is corresponding to the reported values. ${ }^{28}$

${ }^{28}$ S. Alazet, J. Preindl, R. Simonet-Davin, S. Nicolai, A. Nanchen, T. Meyer, J. Waser, J. Org. Chem. 2018, 83, $12334-12356$. 


\section{1-azido-2-tosyl-1,2-dihydro-3H-1 $\lambda^{3}$-benzo[d][1,2]iodazol-3-one (ABZ, 3)}

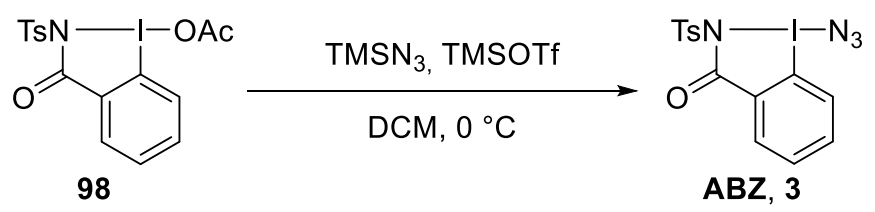

Caution: For safety reasons, the reaction was carried out behind an antiblast shield. Following a reported procedure, ${ }^{28}$ TMSOTf $(14 \mu \mathrm{L}, 0.080 \mathrm{mmol}, 0.005$ equiv) was added dropwise to a suspension of 98 (7.13 g, $15.5 \mathrm{mmol}, 1.0$ equiv.) and $\mathrm{TMSN}_{3}(3.0 \mathrm{~mL}, 23 \mathrm{mmol}, 1.5$ equiv.) in DCM $(31 \mathrm{~mL})$ at $0{ }^{\circ} \mathrm{C}$. The resulting mixture was stirred for $30 \mathrm{~min}$ at $0{ }^{\circ} \mathrm{C}$, and thereafter, the formed solid was filtered, washed with DCM and then pentane, and dried in vacuo to afford ABZ (3) $(5.38 \mathrm{~g}, 12.2 \mathrm{mmol}, 78 \%)$ as a pale yellow solid. ${ }^{1} \mathbf{H}$ NMR (400 MHz, DMSO-d $\left.\boldsymbol{d}_{\boldsymbol{\sigma}}\right) \delta 8.17$ (dd, $J=8.3,0.9 \mathrm{~Hz}, 1 \mathrm{H}, \mathrm{Ar} H), 8.03-7.93(\mathrm{~m}, 2 \mathrm{H}, \mathrm{Ar} H), 7.93-7.87$ (m, 2H, $\mathrm{ArH}), 7.75(\mathrm{td}, J=7.4,0.9$ $\mathrm{Hz}, 1 \mathrm{H}, \operatorname{Ar} H$ ), $7.46-7.37$ (m, 2H, $\mathrm{Ar} H$ ), 2.38 (s, 3H, CH ); ${ }^{13} \mathbf{C}$ NMR (400 MHz, DMSO-d 6 ) $\delta$ $162.6,144.0,136.7,136.3,132.5,131.2,131.2,129.5,128.5,127.8,118.2,21.1$. The characterization data is corresponding to the reported values. ${ }^{28}$ 


\section{Radical Amination of Cyclopropenes for the Synthesis of Alkenyl Nitriles}

\section{General procedure $\mathbf{C}$}

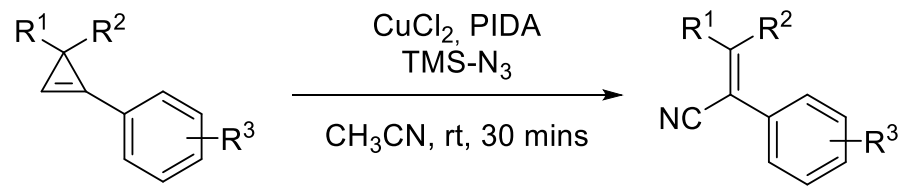

A $5 \mathrm{~mL}$ test tube was charged with $\mathrm{CuCl}_{2}(2 \mathrm{mg}, 0.05 \mathrm{mmol}, 0.05$ equiv.), the cyclopropene ( 0.30 mmol, 1.0 equiv.) and PIDA (174 $\mathrm{mg}, 0.540 \mathrm{mmol}, 1.8$ equiv.). The tube was sealed, evacuated and back-filled with nitrogen ( 3 times). Then $\mathrm{CH}_{3} \mathrm{CN}(3 \mathrm{~mL}, 0.1 \mathrm{M})$ and TMS- $\mathrm{N}_{3}(72 \mu \mathrm{L}, 0.54$ mmol, 1.8 equiv.) were added via syringe and the resulting mixture was stirred at room temperature for 30 minutes. The reaction mixture was then filtered through a small pad of silica gel eluting with $\mathrm{Et}_{2} \mathrm{O}$, and the filtrate was concentrated under reduced pressure. The obtained crude residue was then subjected to column chromatography using the indicated solvents.

\section{Dimethyl 2-(cyano(phenyl)methylene)malonate (2)}

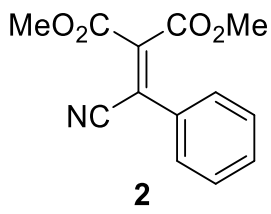

Following the general procedure $\mathrm{C}$, starting from dimethyl 2-phenylcycloprop-2-ene-1,1dicarboxylate (1) $(70 \mathrm{mg}, 0.30 \mathrm{mmol}, 1.0$ equiv.), the title compound 2 was obtained after purification by column chromatography on Biotage (Büchi flashpure cartridge $25 \mathrm{~g}$, Pentane:EtOAc 80:20) as a grey solid $\left(67 \mathrm{mg}, 0.27 \mathrm{mmol}, 91 \%\right.$ yield). $\mathbf{R}_{f} 0.33$ (Pentane:EtOAc 80:20); m.p = 74-75 ${ }^{\circ} \mathrm{C} ;{ }^{1} \mathbf{H}$ NMR (400 MHz, Chloroform- $\left.\boldsymbol{d}\right) \delta 7.55-7.38(\mathrm{~m}, 5 \mathrm{H}, \operatorname{Ar} H), 3.94$ $\left(\mathrm{s}, 3 \mathrm{H}, \mathrm{CO}_{2} \mathrm{CH}_{3}\right), 3.70\left(\mathrm{~s}, 3 \mathrm{H}, \mathrm{CO}_{2} \mathrm{CH}_{3}\right) ;{ }^{13} \mathbf{C}$ NMR (101 MHz, Chloroform-d) $\delta$ 164.2, 161.7, 137.0, 132.0, 131.6, 129.3, 128.3, 125.8, 115.6, 53.7, 53.3; IR $\widetilde{v}_{\max } 2956(\mathrm{w}), 1737(\mathrm{~s}), 1608(\mathrm{w})$, $1436(\mathrm{~m}), 1309(\mathrm{~m}), 1256(\mathrm{~s}), 1229(\mathrm{~s}), 1187(\mathrm{~m}), 1098(\mathrm{~m}), 1021(\mathrm{~m}), 982(\mathrm{w}), 929(\mathrm{w}), 862(\mathrm{w})$, 768 (s), 697 (m); HRMS (ESI) calcd for $\mathrm{C}_{13} \mathrm{H}_{12} \mathrm{NO}_{4}{ }^{+}[\mathrm{M}+\mathrm{H}]^{+}$246.0761; found 246.0764.

\section{Dibenzyl 2-(cyano(phenyl)methylene)malonate (4)}

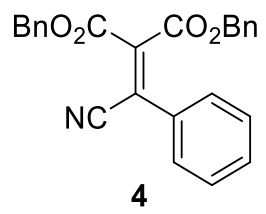

Following the general procedure $\mathrm{C}$, starting from dibenzyl 2-phenylcycloprop-2-ene-1,1dicarboxylate (65) (115 mg, $0.300 \mathrm{mmol}, 1.0$ equiv.), the title compound 4 was obtained after purification by column chromatography on Biotage (Büchi flashpure cartridge $25 \mathrm{~g}$, Pentane:EtOAc 90:10) as a colorless oil ( $88 \mathrm{mg}, 0.22 \mathrm{mmol}, 74 \%$ yield). $\mathbf{R}_{f} 0.52$ (Pentane:EtOAc 
80:20); ${ }^{1}$ H NMR (400 MHz, Chloroform-d) $\delta 7.46-7.40$ (m, 3H, ArH), 7.37 (m, 5H, ArH), 7.33 - 7.29 (m, 3H, ArH), 7.27 - 7.24 (m, 2H, ArH), $7.10-7.05$ (m, 2H, ArH), 5.35 (s, 2H, CH $H_{2}, 5.10$ (s, 2H, $\left.\mathrm{CH}_{2}\right) ;{ }^{13} \mathbf{C}$ NMR (101 MHz, Chloroform-d) $\delta$ 163.4, 161.0, 137.2, 134.6, 134.1, 131.9, $131.5,129.2,128.9,128.9,128.8,128.8,128.7,128.6,128.4,126.0,115.6,68.5,68.4$; IR $\widetilde{v}_{\max }$ 3655 (w), 3453 (w), 3064 (m), 3035 (m), 2951 (w), $2113(\mathrm{w}), 1959$ (w), 1733 (s), $1605(\mathrm{w}), 1496$ (w), $1451(\mathrm{~m}), 1377(\mathrm{w}), 1303$ (m), 1214 (s), $1095(\mathrm{~m}), 1014(\mathrm{~m}), 975(\mathrm{w}), 955(\mathrm{~m}), 907(\mathrm{w}), 738$ (s), 694 (s), 638 (w); HRMS (ESI) calcd for $\mathrm{C}_{25} \mathrm{H}_{19} \mathrm{NNaO}_{4}{ }^{+}[\mathrm{M}+\mathrm{Na}]^{+} 420.1206$; found 420.1206.

Bis(2,2,2-trifluoroethyl) 2-(cyano(phenyl)methylene)malonate (5)

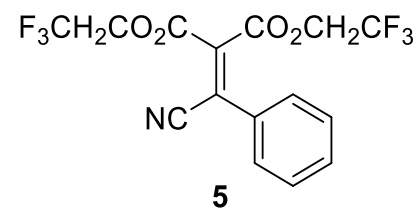

Following the general procedure C, starting from bis(2,2,2-trifluoroethyl) 2-phenylcycloprop-2ene-1,1-dicarboxylate (66) (110 $\mathrm{mg}, 0.300 \mathrm{mmol}, 1.0$ equiv.), the title compound $\mathbf{5}$ was obtained after purification by column chromatography on Biotage (Büchi flashpure cartridge $25 \mathrm{~g}$, Pentane:EtOAc 90:10) as a colorless oil ( $82 \mathrm{mg}, 0.22 \mathrm{mmol}, 72 \%$ yield). $\mathbf{R}_{f} 0.25$ (Pentane:EtOAc 90:10); ${ }^{1} \mathrm{H}$ NMR (400 MHz, Chloroform-d) $\delta 7.59-7.43(\mathrm{~m}, 5 \mathrm{H}, \operatorname{Ar} H), 4.70$ (q, $J=8.1,2 \mathrm{H}$, $\mathrm{CH}_{2} \mathrm{CF}_{3}$ ), 4.49 (q, $\left.J=8.2,2 \mathrm{H}, \mathrm{CH}_{2} \mathrm{CF}_{3}\right) ;{ }^{13} \mathrm{C}$ NMR (101 MHz, Chloroform-d) $\delta$ 161.6, 159.2, $133.5,132.5,131.3,129.7,129.6,128.4,122.4$ (q, $J=277.3 \mathrm{~Hz}), 122.2$ (q, $J=277.6 \mathrm{~Hz}), 114.8$, $62.0(\mathrm{q}, J=37.8 \mathrm{~Hz}), 61.7(\mathrm{q}, J=37.6 \mathrm{~Hz}) ;{ }^{19} \mathbf{F}$ NMR (376 MHz, Chloroform- $d$ ) $\delta=-73.5,-73.6$; IR $\widetilde{\boldsymbol{v}}_{\max } 2981(\mathrm{w}), 1757(\mathrm{~m}), 1608(\mathrm{w}), 1576(\mathrm{w}), 1446(\mathrm{w}), 1413(\mathrm{~m}), 1287$ (s), 1210 (s), 1166 (s), $1100(\mathrm{~m}), 1050$ (m), 1018 (w), $980(\mathrm{~m}), 846$ (w), 766 (m), 697 (m), $656(\mathrm{~m})$; HRMS (APPI) calcd for $\mathrm{C}_{15} \mathrm{H}_{10} \mathrm{~F}_{6} \mathrm{NO}_{4}{ }^{+}[\mathrm{M}+\mathrm{H}]^{+}$382.0509; found 382.0521 .

\section{Dimethyl 2-((4-bromophenyl)(cyano)methylene)malonate (6)}

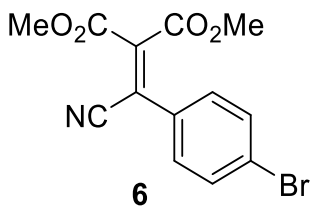

Following the general procedure $\mathrm{C}$, starting from dimethyl 2-(4-bromophenyl)cycloprop-2-ene1,1-dicarboxylate (67) (93 $\mathrm{mg}, 0.30 \mathrm{mmol}, 1.0$ equiv.), the title compound $\mathbf{6}$ was obtained after purification by column chromatography on Biotage (Büchi flashpure cartridge $25 \mathrm{~g}$, Pentane:EtOAc 85:15) as a white solid ( $86 \mathrm{mg}, 0.27 \mathrm{mmol}, 88 \%$ yield). $\mathbf{R}_{f} 0.30$ (Pentane:EtOAc 80:20); m.p = 66-67 ${ }^{\circ} \mathrm{C}$; ${ }^{1} \mathbf{H}$ NMR (400 MHz, Chloroform-d) $\delta 7.63-7.55$ (m, 2H, ArH), $7.41-$ $7.33(\mathrm{~m}, 2 \mathrm{H}, \mathrm{ArH}), 3.94\left(\mathrm{~s}, 3 \mathrm{H}, \mathrm{CO}_{2} \mathrm{CH}_{3}\right), 3.72\left(\mathrm{~s}, 3 \mathrm{H}, \mathrm{CO}_{2} \mathrm{CH}_{3}\right) ;{ }^{13} \mathrm{C}$ NMR (101 MHz, Chloroform- $d$ ) $\delta 163.9,161.5,137.4,132.6,130.8,129.8,126.5,124.6,115.3,53.8,53.5$; IR $\widetilde{v}_{\max }$ 3664 (w), 2960 (m), 2902 (w), 1736 (s), 1593 (m), 1485 (m), 1441 (m), 1399 (m), 1308 (s), 1232 (s), 1083 (s), 1020 (m), 981 (m), 928 (w), 832 (m), 774 (m), 722 (m), 654 (w); HRMS (ESI) calcd for $\mathrm{C}_{13} \mathrm{H}_{11} \mathrm{BrNO}_{4}{ }^{+}[\mathrm{M}+\mathrm{H}]^{+}$323.9866; found 323.9874 . 
Dimethyl 2-((4-(tert-butyl)phenyl)(cyano)methylene)malonate (7)<smiles>COC(=O)C(C#N)=C(C#N)c1ccc(Br)cc1</smiles>

Following the general procedure $\mathrm{C}$, starting from dimethyl 2-(4-(tert-butyl)phenyl)cycloprop-2ene-1,1-dicarboxylate (68) (87 mg, $0.30 \mathrm{mmol}, 1.0$ equiv.), the title compound 7 was obtained after purification by column chromatography on Biotage (Büchi flashpure cartridge $25 \mathrm{~g}$, Pentane:EtOAc 90:10) as a colorless oil ( $86 \mathrm{mg}, 0.29 \mathrm{mmol}, 95 \%$ yield). $\mathbf{R}_{f} 0.48$ (Pentane:EtOAc 80:20); ${ }^{1} \mathbf{H}$ NMR (400 MHz, Chloroform-d) $\delta 7.45$ (d, $J=1.0 \mathrm{~Hz}, 4 \mathrm{H}, \operatorname{Ar} H$ ), 3.92 (s, 3H, $\left.\mathrm{CO}_{2} \mathrm{CH}_{3}\right), 3.73$ (s, $\left.3 \mathrm{H}, \mathrm{CO}_{2} \mathrm{CH}_{3}\right), 1.32\left(\mathrm{~s}, 9 \mathrm{H},{ }^{t} \mathrm{Bu}\right) ;{ }^{13} \mathbf{C}$ NMR (101 MHz, Chloroform-d) $\delta 164.5$, $161.8,155.4,135.8,129.0,128.3,126.3,125.8,115.7,53.6,53.3,35.1,31.1$; IR $\widetilde{v}_{\max } 3670(\mathrm{w})$, 3461 (w), 2960 (m), 2872 (w), 2224 (w), 2120 (w), 1737 (s), 1601 (m), 1510 (w), 1308 (m), 1257 (s), 1092 (s), 1026 (m), 984 (w), 930 (w), 840 (m), 737 (s), 663 (w); HRMS (ESI) calcd for $\mathrm{C}_{17} \mathrm{H}_{19} \mathrm{NNaO}_{4}{ }^{+}[\mathrm{M}+\mathrm{Na}]^{+}$324.1206; found 324.1208.

\section{Dimethyl 2-(cyano(3-fluorophenyl)methylene)malonate (8)}

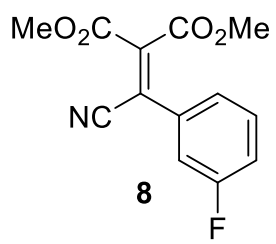

Following the general procedure $\mathrm{C}$, starting from dimethyl 2-(3-fluorophenyl)cycloprop-2-ene-1,1dicarboxylate (70) $(75 \mathrm{mg}, 0.30 \mathrm{mmol}, 1.0$ equiv.), the title compound 8 was obtained after purification by column chromatography on Biotage (Büchi flashpure cartridge $25 \mathrm{~g}$, Pentane:EtOAc 85:15) as a white solid (67 mg, $0.26 \mathrm{mmol}, 81 \%$ yield, $96 \%$ purity). ${ }^{29} \mathbf{R}_{f} 0.38$ (Pentane:EtOAc 80:20); m.p = 62-63 ${ }^{\circ} \mathrm{C} ;{ }^{1} \mathbf{H}$ NMR (400 MHz, Chloroform- $\left.d\right) \delta 7.47-7.39(\mathrm{~m}$, $1 \mathrm{H}, \mathrm{ArH}), 7.32-7.27(\mathrm{~m}, 1 \mathrm{H}, \mathrm{ArH}), 7.24-7.15$ (m, 2H, $\mathrm{ArH}), 3.94$ (s, 3H, $\left.\mathrm{CO}_{2} \mathrm{CH}_{3}\right), 3.72$ (s, $3 \mathrm{H}$, $\left.\mathrm{CO}_{2} \mathrm{CH}_{3}\right) ;{ }^{13} \mathrm{C}$ NMR (101 MHz, Chloroform-d) $\delta 163.7,162.7(\mathrm{~d}, J=249.5 \mathrm{~Hz}), 161.4,138.0$, $133.7(\mathrm{~d}, J=8.1 \mathrm{~Hz}), 131.1(\mathrm{~d}, J=8.4 \mathrm{~Hz}), 124.2,124.2(\mathrm{~d}, J=3.6 \mathrm{~Hz}), 118.7(\mathrm{~d}, J=21.2 \mathrm{~Hz})$, $115.5(\mathrm{~d}, J=23.9 \mathrm{~Hz}), 115.2,53.8,53.5 ;{ }^{19} \mathbf{F}$ NMR $\left(376 \mathrm{MHz}\right.$, Chloroform-d) $\delta-110.3$; IR $\widetilde{\boldsymbol{v}}_{\max }$ 3077 (w), 3012 (w), 2959 (w), 2849 (w), 1738 (s), $1587(\mathrm{~m}), 1484(\mathrm{w}), 1440(\mathrm{~m}), 1317(\mathrm{~m}), 1242$ (s), 1191 (m), 1099 (m), 1031 (m), 928 (m), 840 (w), 792 (m), 697 (m), 652 (w); HRMS (ESI) calcd for $\mathrm{C}_{13} \mathrm{H}_{10} \mathrm{FNNaO}_{4}{ }^{+}[\mathrm{M}+\mathrm{Na}]^{+} 286.0486$; found 286.0492 .

\footnotetext{
${ }^{29}$ For characterization purposes, a small sample of the product was re-purified.
} 
Dimethyl 2-(cyano(naphthalen-1-yl)methylene)malonate (9)<smiles>COC(=O)C(C(C)=O)=C(C#N)c1cccc2ccccc12</smiles>

Following the general procedure $\mathrm{C}$, starting from dimethyl 2-(naphthalen-1-yl)cycloprop-2-ene1,1-dicarboxylate (71) (85 mg, $0.30 \mathrm{mmol}, 1.0$ equiv.), the title compound 9 was obtained after purification by column chromatography on Biotage (Büchi flashpure cartridge $25 \mathrm{~g}$, Pentane:EtOAc 85:15) as a yellow solid ( $83 \mathrm{mg}, 0.28 \mathrm{mmol}, 94 \%$ yield). $\mathbf{R}_{f} 0.35$ (Pentane:EtOAc 80:20); m.p = 138-139 ${ }^{\circ} \mathrm{C}$; ${ }^{1} \mathbf{H}$ NMR (400 MHz, Chloroform-d) $\delta 7.97-7.87$ (m, 3H, ArH), 7.64 - $7.55(\mathrm{~m}, 2 \mathrm{H}, \mathrm{ArH}), 7.50-7.40(\mathrm{~m}, 2 \mathrm{H}, \mathrm{ArH}), 4.00\left(\mathrm{~s}, 3 \mathrm{H}, \mathrm{CO}_{2} \mathrm{CH}_{3}\right), 3.43\left(\mathrm{~s}, 3 \mathrm{H}, \mathrm{CO}_{2} \mathrm{CH}_{3}\right) ;{ }^{13} \mathrm{C}$ NMR (101 MHz, Chloroform-d) $\delta$ 163.2, 161.6, 140.5, 133.6, 131.5, 130.1, 129.0, 128.9, 127.8, 127.2, 127.1, 125.1, 125.0, 124.4, 115.1, 53.7, 53.1; IR $\widetilde{\boldsymbol{v}}_{\max } 3135$ (w), 3025 (w), 2951 (w), 1718 (s), 1601 (w), 1492 (m), 1445 (m), 1434 (m), 1288 (m), 1212 (s), 1112 (w), 1073 (w), 1020 (m), 763 (m), 699 (s); HRMS (ESI) calcd for $\mathrm{C}_{17} \mathrm{H}_{14} \mathrm{NO}_{4}{ }^{+}[\mathrm{M}+\mathrm{H}]^{+} 296.0917$; found 296.0911.

\section{Dimethyl 2-(cyano(2-(trifluoromethyl)phenyl)methylene)malonate (10)}<smiles>COC(=O)/C(C#N)=C(\C(C)=O)c1ccccc1C(F)(F)F</smiles>

Following the general procedure $\mathrm{C}$, starting from dimethyl 2-(2-(trifluoromethyl)phenyl)cycloprop-2-ene-1,1-dicarboxylate (72) (90 mg, $0.30 \mathrm{mmol}, 1.0$ equiv.), the title compound 10 was obtained after purification by column chromatography on Biotage (Büchi flashpure cartridge $25 \mathrm{~g}$, Pentane:EtOAc 80:20) as a colorless oil (72 $\mathrm{mg}, 0.23 \mathrm{mmol}, 77 \%$ yield). $\mathbf{R}_{f} 0.30$ (Pentane:EtOAc 80:20); ${ }^{1} \mathbf{H}$ NMR (400 MHz, Chloroform-d) $\delta 7.80-7.73$ (m, 1H, ArH), $7.66-7.57$ (m, 2H, $\mathrm{ArH}), 7.39-7.32(\mathrm{~m}, 1 \mathrm{H}, \mathrm{ArH}), 3.98\left(\mathrm{~s}, 3 \mathrm{H}, \mathrm{CO}_{2} \mathrm{CH}_{3}\right), 3.56\left(\mathrm{~s}, 3 \mathrm{H}, \mathrm{CO}_{2} \mathrm{CH}_{3}\right) ;{ }^{13} \mathrm{C}$ NMR (101 MHz, Chloroform- $d$ ) $\delta 162.0,161.6,140.6,132.4,130.8,130.1,129.4$ (q, $J=2.6 \mathrm{~Hz}), 128.6$ (q, $J=31.6 \mathrm{~Hz}), 127.3(\mathrm{q}, J=4.7 \mathrm{~Hz}), 123.5,123.4(\mathrm{q}, J=274.0 \mathrm{~Hz}), 114.2,53.9,53.2 ;{ }^{19} \mathbf{F ~ N M R}$ (376 MHz, Chloroform-d) $\delta$-59.0; IR $\widetilde{\boldsymbol{v}}_{\max } 3012(\mathrm{w}), 2960(\mathrm{w}), 2850(\mathrm{w}), 1740(\mathrm{~s}), 1623(\mathrm{w})$, 1442 (m), 1313 (s), 1261 (s), 1176 (s), 1121 (s), 1060 (m), 1029 (m), 978 (m), 928 (w), 766 (s), $707(\mathrm{w}), 659$ (m); HRMS (ESI) calcd for $\mathrm{C}_{14} \mathrm{H}_{11} \mathrm{~F}_{3} \mathrm{NO}_{4}{ }^{+}[\mathrm{M}+\mathrm{H}]^{+}$314.0635; found 314.0640.

\section{Methyl 3-cyano-2,3-diphenylacrylate (14)}<smiles>COC(=O)/C(=C(\C#N)c1ccccc1)c1ccccc1</smiles> 
Following the general procedure $\mathrm{C}$, starting from methyl 1,2-diphenylcycloprop-2-enecarboxylate (39) (400 $\mathrm{mg}, 1.60 \mathrm{mmol}, 1.0$ equiv.), the title compound $\mathbf{1 4}$ was obtained after purification by column chromatography on Biotage (Büchi flashpure cartridge $40 \mathrm{~g}$, Pentane:EtOAc 90:10) as a white solid (370 $\mathrm{mg}, 1.41 \mathrm{mmol}, 84 \%$ yield, $95 \%$ purity) and as an inseparable 1:1.18 mixture of unassigned Z/E isomers. ${ }^{29} \mathbf{R}_{f} 0.32$ (Pentane:EtOAc 90:10); $\mathbf{m . p}=85-86{ }^{\circ} \mathrm{C} ;{ }^{1} \mathbf{H}$ NMR (400 MHz, Chloroform- $\boldsymbol{d}$ ) $\delta$ (Major isomer) $7.64-7.62(\mathrm{~m}, 1 \mathrm{H}, \mathrm{ArH}), 7.49-7.46(\mathrm{~m}, 2 \mathrm{H}, \mathrm{ArH}), 7.45-$ $7.44(\mathrm{~m}, 1 \mathrm{H}, \operatorname{Ar} H), 7.26-7.23(\mathrm{~m}, 3 \mathrm{H}, \operatorname{Ar} H), 7.22-7.21(\mathrm{~m}, 2 \mathrm{H}, \operatorname{Ar} H), 7.15-7.13(\mathrm{~m}, 1 \mathrm{H}, \operatorname{Ar} H)$, $3.93\left(\mathrm{~s}, 3 \mathrm{H}, \mathrm{CO}_{2} \mathrm{CH}_{3}\right) ;{ }^{1} \mathbf{H}$ NMR (400 MHz, Chloroform-d) $\delta$ (Minor isomer) $7.65-7.63(\mathrm{~m}, 1 \mathrm{H}$, $\operatorname{Ar} H), 7.53-7.49(\mathrm{~m}, 2 \mathrm{H}, \operatorname{Ar} H), 7.43-7.42(\mathrm{~m}, 1 \mathrm{H}, \operatorname{Ar} H), 7.33-7.26(\mathrm{~m}, 3 \mathrm{H}, \operatorname{Ar} H), 7.24-7.22$ $(\mathrm{m}, 2 \mathrm{H}, \operatorname{Ar} H), 7.13-7.11(\mathrm{~m}, 1 \mathrm{H}, \operatorname{Ar} H), 3.65\left(\mathrm{~s}, 3 \mathrm{H}, \mathrm{CO}_{2} \mathrm{CH}_{3}\right) ;{ }^{13} \mathrm{C}$ NMR (101 MHz, Chloroform-d) $\delta$ (Major isomer) 166.7, 146.7, 133.3, 132.6, 130.1, 129.7, 129.6, 129.1, 128.7, 128.1, 118.7, 117.7, 53.4; ${ }^{13} \mathbf{C}$ NMR (101 MHz, Chloroform-d) $\delta$ (Minor isomer) 167.5, 148.3, 133.7, 133.5, 130.7, 129.7, 129.6, 129.1, 128.7, 128.4, 117.7, 116.0, 53.0; IR $\widetilde{v}_{\max } 3060$ (w), 2953 (w), $2217(\mathrm{w}), 2111(\mathrm{w}), 1729(\mathrm{~s}), 1592(\mathrm{w}), 1494(\mathrm{w}), 1445(\mathrm{~m}), 1326(\mathrm{~m}), 1306(\mathrm{~m}), 1255(\mathrm{~s})$, 1211 (s), 1084 (w), 1065 (w), 1013 (m), 986 (w), 924 (w), 852 (w), 762 (m), 738 (s), 698 (s), 648 (w); HRMS (ESI) calcd for $\mathrm{C}_{17} \mathrm{H}_{14} \mathrm{NO}_{2}{ }^{+}[\mathrm{M}+\mathrm{H}]^{+} 264.1019$; found 264.1019 . 


\section{Gram-scale Synthesis of 2 and Product Modifications of 2 and 14}

Procedure for the gram scale radical amination of cyclopropene 1

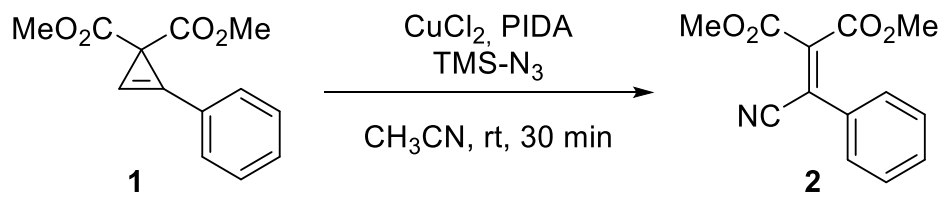

A $100 \mathrm{~mL}$ round bottom flask was charged with $\mathrm{CuCl}_{2}(29 \mathrm{mg}, 0.22 \mathrm{mmol}, 0.05$ equiv.), dimethyl 2-phenylcycloprop-2-ene-1,1-dicarboxylate (1) (1.00 g, $4.31 \mathrm{mmol}, 1.0$ equiv.) and PIDA (2.49 g, $7.75 \mathrm{mmol}, 1.8$ equiv.). The flask was sealed, evacuated and back-filled with nitrogen ( 3 times). Then $\mathrm{CH}_{3} \mathrm{CN}(43 \mathrm{~mL}, 0.1 \mathrm{M})$ and $\mathrm{TMS}-\mathrm{N}_{3}(1.02 \mathrm{~mL}, 7.75 \mathrm{mmol}, 1.8$ equiv.) were added via syringe and the resulting mixture was stirred at room temperature for 30 minutes. The reaction mixture was then filtered through a small pad of silica gel eluting with $\mathrm{Et}_{2} \mathrm{O}$, and the filtrate was concentrated under reduced pressure. The crude residue was purified by column chromatography $\left(\mathrm{SiO}_{2}\right.$, Pentane:EtOAc 80:20) affording 2 (971 mg, $3.96 \mathrm{mmol}, 92 \%$ yield) as a grey solid. Characterization as described in section 4 .

\section{Dimethyl 2-cyano-2-phenylcyclopropane-1,1-dicarboxylate (15)}

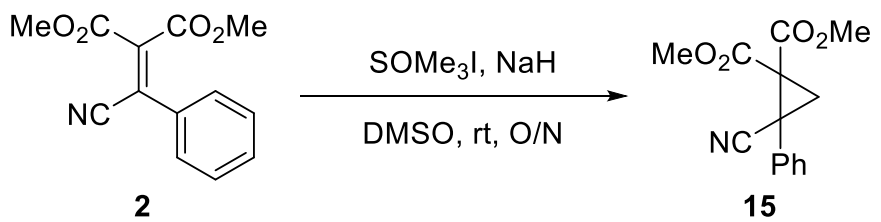

$\mathrm{NaH}$ (9.6 mg, $0.24 \mathrm{mmol}, 1.2$ equiv.) and $\mathrm{SOMe}_{3} \mathrm{I}(62 \mathrm{mg}, 0.28 \mathrm{mmol}, 1.4$ equiv.) were placed in a $5 \mathrm{~mL}$ microwave vial. DMSO $(1.0 \mathrm{~mL})$ was then added and the reaction mixture was stirred until gas evolution has ceased and the mixture was homogeneous. At this point a solution of dimethyl 2-(cyano(phenyl)methylene)malonate (2) $(49 \mathrm{mg}, 0.20 \mathrm{mmol}, 1.0$ equiv.) in DMSO (1.0 mL) was added via syringe and the resulting mixture was left stirring for 3 hours at room temperature. It was then quenched with water $(2 \mathrm{~mL})$, and extracted with $\mathrm{Et}_{2} \mathrm{O}(2 \times 10 \mathrm{~mL})$. The aqueous layer was further washed with $\mathrm{Et}_{2} \mathrm{O}(2 \times 10 \mathrm{~mL})$ and the combined organic layers were washed with water $(5$ x $10 \mathrm{~mL})$ and brine $(10 \mathrm{~mL})$. The combined organic layers were dried over $\mathrm{MgSO}_{4}$, filtered, and concentrated under reduced pressure. The crude residue was purified by column chromatography $\left(\mathrm{SiO}_{2}\right.$, Pentane:EtOAc 75:25) affording $15\left(28 \mathrm{mg}, 0.11 \mathrm{mmol}, 54 \%\right.$ yield) as a colorless oil. $\mathbf{R}_{f}$ 0.32 (Pentane:EtOAc 75:25); ${ }^{1} \mathbf{H}$ NMR (400 MHz, Chloroform-d) $\delta 7.42-7.33$ (m, 5H, $\operatorname{Ar} H$ ), $3.91\left(\mathrm{~s}, 3 \mathrm{H}, \mathrm{CO}_{2} \mathrm{CH}_{3}\right), 3.35$ (s, $\left.3 \mathrm{H}, \mathrm{CO}_{2} \mathrm{CH}_{3}\right), 2.60\left(\mathrm{~d}, J=6.2 \mathrm{~Hz}, 1 \mathrm{H}, \mathrm{CH}_{2}\right), 2.37$ (d, $J=6.2 \mathrm{~Hz}$, $\left.1 \mathrm{H}, \mathrm{CH}_{2}\right) .{ }^{13} \mathbf{C}$ NMR (101 MHz, Chloroform-d) $\delta$ 166.2, 164.5, 130.6, 129.6, 129.0, 128.9, 118.3, 53.8, 53.1, 42.2, 28.2, 22.7; IR $\widetilde{v}_{\max } 2956$ (w), 2241 (w), 1739 (s), 1495 (w), 1438 (m), 1341 (s), 1262 (s), 1233 (s), 1196 (w), 1078 (w), 983 (w), 906 (w), 735 (m), 699 (m); HRMS (ESI) calcd for $\mathrm{C}_{14} \mathrm{H}_{14} \mathrm{NO}_{4}{ }^{+}[\mathrm{M}+\mathrm{H}]^{+} 260.0917$; found 260.0929 . 


\section{3-Ethyl 1,1-dimethyl 2-cyano-2-phenylcyclopent-3-ene-1,1,3-tricarboxylate (17)}

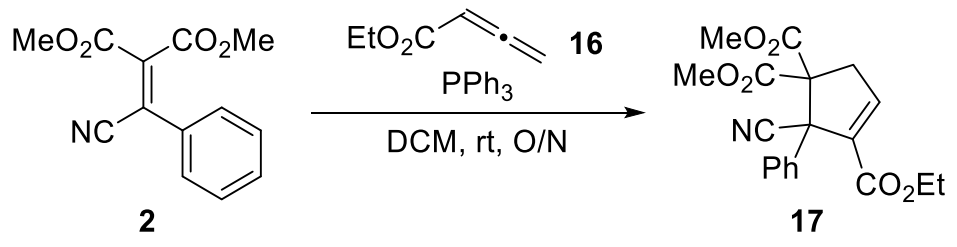

A $5 \mathrm{~mL}$ test tube was charged with $\mathrm{PPh}_{3}(11 \mathrm{mg}, 0.040 \mathrm{mmol}, 0.2$ equiv.) and dimethyl 2(cyano(phenyl)methylene)malonate (2) (49 mg, $0.20 \mathrm{mmol}, 1.0$ equiv.). The tube was sealed, evacuated and back-filled with nitrogen ( 3 times). Then DCM $(2.0 \mathrm{~mL})$ and ethyl buta-2,3-dienoate (16) $(46 \mu \mathrm{L}, 0.40 \mathrm{mmol}, 2.0$ equiv.) were added via syringe, and the resulting reaction mixture was left stirring overnight at room temperature. The mixture was then filtered through a small plug of celite eluting with EtOAc, and the filtrate was concentrated under reduced pressure. The crude residue was purified by column chromatography $\left(\mathrm{SiO}_{2}\right.$, Pentane:EtOAc 90:10 to 70:30) affording 17 (64 mg, $0.18 \mathrm{mmol}, 89 \%$ yield) as a colorless oil. $\mathbf{R}_{\boldsymbol{f}} 0.38$ (Pentane:EtOAc 70:30); ${ }^{\mathbf{1}} \mathbf{H}$ NMR (400 MHz, Chloroform-d) $\delta 7.44-7.38(\mathrm{~m}, 2 \mathrm{H}, \mathrm{ArH}), 7.37-7.30$ (m, 3H, ArH), 7.19 (t, $J=2.6$ $\mathrm{Hz}, 1 \mathrm{H}, \mathrm{C}=\mathrm{CH}), 4.11\left(\mathrm{q}, J=7.2 \mathrm{~Hz}, 2 \mathrm{H}, \mathrm{CH}_{2} \mathrm{CH}_{3}\right), 3.84\left(\mathrm{~s}, 3 \mathrm{H}, \mathrm{CO}_{2} \mathrm{CH}_{3}\right), 3.51$ (dd, $J=19.3,2.5$ $\mathrm{Hz}, 1 \mathrm{H}, \mathrm{CH}_{2}$ ), 3.27 (s, 3H, $\left.\mathrm{CO}_{2} \mathrm{CH}_{3}\right), 3.03$ (dd, $\left.J=19.4,2.9 \mathrm{~Hz}, 1 \mathrm{H}, \mathrm{CH}_{2}\right), 1.11(\mathrm{t}, J=7.1 \mathrm{~Hz}, 3 \mathrm{H}$, $\left.\mathrm{CH}_{2} \mathrm{CH}_{3}\right) ;{ }^{13} \mathbf{C}$ NMR (101 MHz, Chloroform-d) $\delta 169.1,167.3,161.5,144.2,135.8,133.5,129.1$, 128.7, 127.0, 118.7, 69.8, 61.3, 58.2, 53.6, 52.8, 39.4, 13.9 ; IR $\widetilde{\boldsymbol{v}}_{\max } 2985$ (w), $2956(\mathrm{w}), 2253(\mathrm{w})$, 1739 (s), 1719 (s), 1642 (w), 1495 (w), 1448 (m), 1436 (m), 1371 (m), 1331 (m), 1267 (s), 1247 (s), $1196(\mathrm{~m}), 1159(\mathrm{~m}), 1137(\mathrm{~m}), 1110(\mathrm{~m}), 1092(\mathrm{~m}), 1028(\mathrm{~m}), 957(\mathrm{w}), 914(\mathrm{~m}), 863(\mathrm{w}), 841$ (w), $775(\mathrm{~m}), 733$ (s), $700(\mathrm{~m}), 647(\mathrm{w})$; HRMS (ESI) calcd for $\mathrm{C}_{19} \mathrm{H}_{19} \mathrm{NNaO}_{6}{ }^{+}[\mathrm{M}+\mathrm{Na}]^{+} 380.1105$; found 380.1109 .

\section{Dimethyl 1-cyano-4-oxo-3,4-dihydro-[1,1'-biphenyl]-2,2(1H)-dicarboxylate (19) and dimethyl 1-cyano-5-oxo-5,6-dihydro-[1,1'-biphenyl]-2,2(1H)-dicarboxylate (99)}
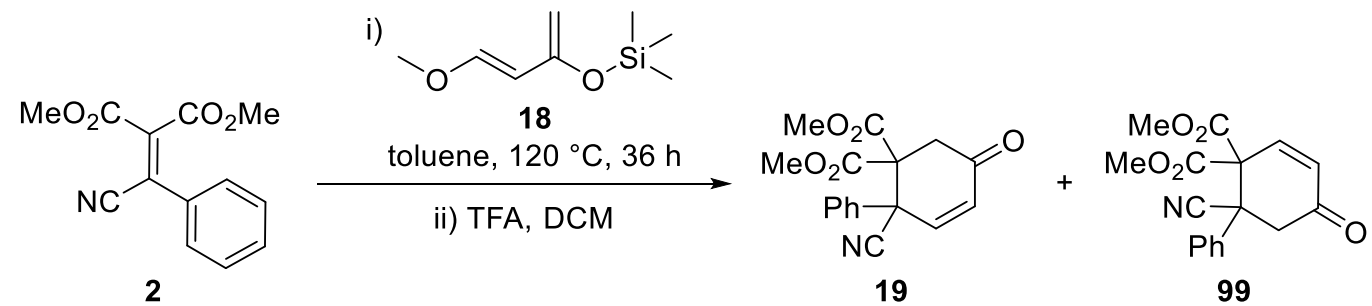

To a solution of dimethyl 2-(cyano(phenyl)methylene)malonate (2) (49 mg, $0.20 \mathrm{mmol}, 1.0$ equiv.) in toluene $(2 \mathrm{~mL})$ was added $(E)$-((4-methoxybuta-1,3-dien-2-yl)oxy)trimethylsilane $(\mathbf{1 8})(81 \mu \mathrm{L}$, $0.40 \mathrm{mmol}, 2.0$ equiv.) and the resulting reaction mixture was stirred at $120{ }^{\circ} \mathrm{C}$ for 36 hours. The reaction mixture was then concentrated under reduced pressure. To the residue was added DCM ( 2 $\mathrm{mL}$ ) and one drop of TFA. The reaction mixture was stirred at room temperature for 2 hours and poured into a saturated $\mathrm{NaHCO}_{3}$ solution $(5 \mathrm{~mL})$. The aqueous layer was then extracted with DCM $(3 \times 10 \mathrm{~mL})$ and the combined organic layers were washed with brine $(10 \mathrm{~mL})$, dried over $\mathrm{MgSO}_{4}$, filtered, and concentrated under reduced pressure. The crude residue was purified by column chromatography $\left(\mathrm{SiO}_{2}\right.$, Pentane:EtOAc 75:25) affording 19 and 99 as an inseparable 4.2:1 mixture of putative regioisomers (determined by integration of the olefinic proton peaks in beta position of 
the ketone in ${ }^{1} \mathrm{H}$ NMR) and as a colorless oil (40 mg, $0.13 \mathrm{mmol}, 64 \%$ yield). ${ }^{30} \mathbf{R}_{f} 0.35$ (Pentane:EtOAc 70:30); ${ }^{1} \mathbf{H}$ NMR (400 MHz, Chloroform-d) $\delta$ (Major, 19) $7.51-7.46$ (m, 2H, $\operatorname{ArH}), 7.45-7.41(\mathrm{~m}, 3 \mathrm{H}, \mathrm{ArH}), 6.90(\mathrm{~d}, J=10.1 \mathrm{~Hz}, 1 \mathrm{H}, H \mathrm{C}=\mathrm{CHCO}), 6.41(\mathrm{~d}, J=10.0 \mathrm{~Hz}, 1 \mathrm{H}$, $\mathrm{HC}=\mathrm{CHCO}), 3.80\left(\mathrm{~s}, 3 \mathrm{H}, \mathrm{CO}_{2} \mathrm{CH}_{3}\right), 3.66\left(\mathrm{~s}, 3 \mathrm{H}, \mathrm{CO}_{2} \mathrm{CH}_{3}\right), 3.04\left(\mathrm{~d}, J=17.8 \mathrm{~Hz}, 1 \mathrm{H}, \mathrm{CH}_{2}\right), 2.93$ $\left(\mathrm{d}, J=17.8 \mathrm{~Hz}, 1 \mathrm{H}, \mathrm{CH}_{2}\right)$; ${ }^{1} \mathrm{H}$ NMR (400 MHz, Chloroform-d) $\delta$ (Minor, 98) $7.42-7.39$ (m, 2H, $\operatorname{Ar} H), 7.36-7.31(\mathrm{~m}, 3 \mathrm{H}, \mathrm{ArH}), 7.20(\mathrm{~d}, J=10.4 \mathrm{~Hz}, 1 \mathrm{H}, H \mathrm{C}=\mathrm{CHCO}), 6.36(\mathrm{~d}, J=10.4 \mathrm{~Hz}, 1 \mathrm{H}$, $\mathrm{HC}=\mathrm{CHCO}), 3.86\left(\mathrm{~s}, 3 \mathrm{H}, \mathrm{CO}_{2} \mathrm{CH}_{3}\right), 3.83$ (hidden $\left.d, 1 \mathrm{H}, \mathrm{CH}_{2}\right), 3.64\left(\mathrm{~s}, 3 \mathrm{H}, \mathrm{CO}_{2} \mathrm{CH}_{3}\right), 3.50(\mathrm{~d}, J=$ $\left.23.2 \mathrm{~Hz}, 1 \mathrm{H}, \mathrm{CH}_{2}\right) ;{ }^{13} \mathbf{C}$ NMR (101 MHz, Chloroform-d) $\delta$ (Major, 19) 193.1, 168.0, 166.5, 143.8, 130.1, 129.3, 129.0, 128.4, 127.5, 119.2, 54.0, 53.4, 48.1, 45.5, 39.8; ${ }^{13}$ C NMR (101 MHz, Chloroform- $d$ ) $\delta$ (Minor, 98) 192.6, 168.0, 166.3, 142.0, 135.1, 130.9, 129.4, 129.0, 128.6, 120.4, 62.7, 54.3, 53.7, 47.9, 45.5; IR $\widetilde{\boldsymbol{v}}_{\max } 3060$ (w), 3009 (w), 2957 (w), $2844(\mathrm{w}), 1743$ (s), $1694(\mathrm{~s})$, 1493 (w), 1449 (m), 1436 (m), 1382 (w), 1277 (s), 1239 (s), 1200 (m), 1175 (m), 1137 (w), 1105 (w), 1070 (w), 1053 (w), 1023 (w), 955 (w), 933 (m), 854 (w), 759 (m), 738 (m), 702 (m); HRMS (ESI) calcd for $\mathrm{C}_{17} \mathrm{H}_{15} \mathrm{NNaO}_{5}{ }^{+}[\mathrm{M}+\mathrm{Na}]^{+} 336.0842$; found 336.0840 .

\section{Methyl 10-cyanophenanthrene-9-carboxylate (20)}

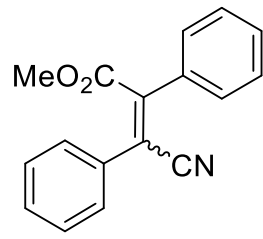

14, Z/E $1: 1.18$

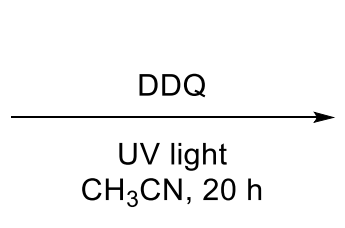

UV light
$\mathrm{CH}_{3} \mathrm{CN}, 20 \mathrm{~h}$

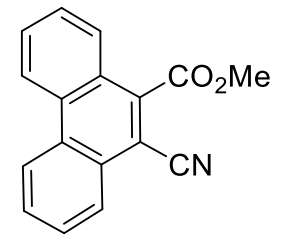

20

A $10 \mathrm{~mL}$ test tube was charged with methyl 3-cyano-2,3-diphenylacrylate (14) (1:1.18 unassigned mixture of $\mathrm{Z} / \mathrm{E}$ isomers, $79 \mathrm{mg}, 0.30 \mathrm{mmol}, 1.0$ equiv.) and DDQ (75 mg, $0.33 \mathrm{mmol}, 1.1 \mathrm{equiv}$.). The tube was sealed, evacuated and back-filled with nitrogen ( 3 times). Then $\mathrm{CH}_{3} \mathrm{CN}(6.0 \mathrm{~mL})$ was added to the reaction mixture before being placed under UV irradiation $(365 \mathrm{~nm})$ in a Rayonet reactor for 20 hours. The reaction mixture was then diluted with EtOAc and concentrated under reduced pressure. The obtained residue was filtered through a small pad of silica gel eluting with a 1:1 mixture of pentane:DCM and the filtrate was evaporated under reduced pressure. The crude residue was purified by column chromatography $\left(\mathrm{SiO}_{2}\right.$, Pentane:EtOAc 90:10) affording 20 (58 $\mathrm{mg}, 0.22 \mathrm{mmol}, 74 \%$ yield) as a white solid. $\mathbf{R}_{\boldsymbol{f}} 0.28$ (Pentane:EtOAc 90:10); m.p = $118-119{ }^{\circ} \mathrm{C}$; ${ }^{1}$ H NMR (400 MHz, Chloroform-d) $\delta 8.76-8.65$ (m, 2H, ArH), $8.42-8.34(\mathrm{~m}, 1 \mathrm{H}, \mathrm{ArH}), 8.10$ (ddd, $J=8.3,1.4,0.6 \mathrm{~Hz}, 1 \mathrm{H}, \mathrm{ArH}), 7.86-7.75$ (m, 3H, ArH), 7.71 (ddd, $J=8.2,7.0,1.2 \mathrm{~Hz}, 1 \mathrm{H}$, $\mathrm{ArH}), 4.19$ (s, 3H, $\left.\mathrm{CO}_{2} \mathrm{CH}_{3}\right) ;{ }^{13} \mathbf{C}$ NMR (101 MHz, Chloroform-d) $\delta 167.0,137.8,131.8,130.5$, $130.2,129.5,128.7,128.3,127.9,127.2,127.1,126.8,123.2,123.2,116.1,109.1,53.6$; IR $\widetilde{\boldsymbol{v}}_{\max }$ $3067(\mathrm{w}), 2954(\mathrm{w}), 2225(\mathrm{~m}), 1731$ (s), $1611(\mathrm{w}), 1526(\mathrm{w}), 1494(\mathrm{w}), 1448(\mathrm{~s}), 1381(\mathrm{~m}), 1331$ (w), 1318 (w), 1298 (w), 1253 (s), 1215 (s), 1153 (m), 1063 (m), 986 (m), 799 (w), 760 (s), 740 (m), 722 (s), $677(\mathrm{w}), 652(\mathrm{w}), 616(\mathrm{w})$; HRMS (ESI) calcd for $\mathrm{C}_{17} \mathrm{H}_{12} \mathrm{NO}_{2}{ }^{+}[\mathrm{M}+\mathrm{H}]^{+} 262.0863$; found 262.0868 .

\footnotetext{
${ }^{30}$ Complete characterization of compound 99 could not be achieved with 2D NMR experiments, the structure proposed here is the one that seemed most likely of the other possible regioisomer, based on ${ }^{1} \mathrm{H}$ and ${ }^{13} \mathrm{C}$ NMR analysis.
} 


\section{Optimization of the One-pot Synthesis of PACs from Cyclopropenes}

General method for the optimization:

A $5 \mathrm{~mL}$ test tube was charged with $\mathrm{CuCl}_{2}(0.7 \mathrm{mg}, 0.05 \mathrm{mmol}, 0.05$ equiv. $)$, methyl 1,2diphenylcycloprop-2-enecarboxylate (39) (25 mg, $0.10 \mathrm{mmol}, 1.0$ equiv.) and PIDA (58 mg, 0.18 mmol, 1.8 equiv.). The tube was sealed, evacuated and back-filled with nitrogen ( 3 times). Then $\mathrm{CH}_{3} \mathrm{CN}(1 \mathrm{~mL}, 0.1 \mathrm{M})$ and TMS- $\mathrm{N}_{3}(24 \mu \mathrm{L}, 0.18 \mathrm{mmol}, 1.8$ equiv.) were added via syringe and the resulting mixture was stirred at room temperature for 30 minutes. Then a solution of DDQ in $\mathrm{CH}_{3} \mathrm{CN}$ was added to the reaction mixture before being placed under UV irradiation ( $365 \mathrm{~nm}$ ) in a Rayonet reactor for 20 hours. The reaction mixture was then diluted with EtOAc and concentrated under reduced pressure. The obtained residue was filtered through a small pad of silica gel eluting with a 1:1 mixture of pentane:DCM and the filtrate was evaporated under reduced pressure. When product 20 was isolated, the obtained crude residue was subjected to purification by column chromatography on Biotage (Büchi flashpure cartridge $25 \mathrm{~g}$, Pentane:EtOAc 90:10).

\section{Table S1: Optimization of the one-pot synthesis of PACs from cyclopropene 39}

\begin{tabular}{|c|c|c|c|c|c|}
\hline X & $\begin{array}{l}\text { i) } \mathrm{C} \\
\mathrm{PID} \\
\mathrm{TMS} \\
\mathrm{CH}_{3} \\
\text { ii) } \mathrm{D} \\
\mathrm{CH} \\
\mathrm{U}\end{array}$ & $\begin{array}{l}(5 \mathrm{~mol} \%) \\
.8 \text { equiv.) } \\
1.8 \text { equiv. }) \\
1 \mathrm{tr}, 30 \mathrm{mins} \\
(\mathrm{X} \text { equiv. }) \\
\mathrm{J}(\mathrm{Y} \mathrm{mL}) \\
\mathrm{ht}, 20 \mathrm{~h}\end{array}$ & & & $\mathrm{MeO}_{2} \mathrm{C}$ \\
\hline Entry & $\mathrm{X}$ equiv. & $\mathbf{Y} \mathbf{~ m L}$ & Yield of $20^{a}$ & Yield of $\mathbf{1 0 0}^{a}$ & Yield of $14^{a}$ \\
\hline 1 & - & - & $42 \%$ & $39 \%$ & - \\
\hline $2^{b}$ & - & - & $61 \%$ & $15 \%$ & - \\
\hline 3 & 1.1 equiv. & $1 \mathrm{~mL}$ & $69 \%$ & - & $22 \%$ \\
\hline 4 & 1.5 equiv. & $1 \mathrm{~mL}$ & $67 \%$ & - & $20 \%$ \\
\hline 5 & 0.5 equiv. & $1 \mathrm{~mL}$ & $60 \%$ & $15 \%$ & $7 \%$ \\
\hline $6^{c}$ & 1.1 equiv. & $1 \mathrm{~mL}$ & $37 \%$ & $32 \%$ & - \\
\hline $7^{d}$ & 1.1 equiv. & $1 \mathrm{~mL}$ & $72 \%$ & - & $16 \%$ \\
\hline $8^{d}$ & 1.1 equiv. & $2 \mathrm{~mL}$ & $82 \%\left(80 \%{ }^{e}\right)$ & - & $10 \%$ \\
\hline
\end{tabular}

(a) Yields estimated by ${ }^{1} \mathrm{H}$ NMR of the crude reaction mixture using dibromomethane as internal standard. (b) 3.0 equivalents of PIDA were used. (c) After the first step, the reaction vessel was placed for $20 \mathrm{~h}$ under UV irradiation, then the diluted DDQ solution was added and the reaction was stopped after $6 \mathrm{~h}$. (d) commercial DDQ recrystallized from distilled $\mathrm{CHCl}_{3}$ was used. (e) Isolated yield on 0.3 mmol. 


\section{One-pot Synthesis of PACs from Cyclopropenes}

\section{General procedure D}
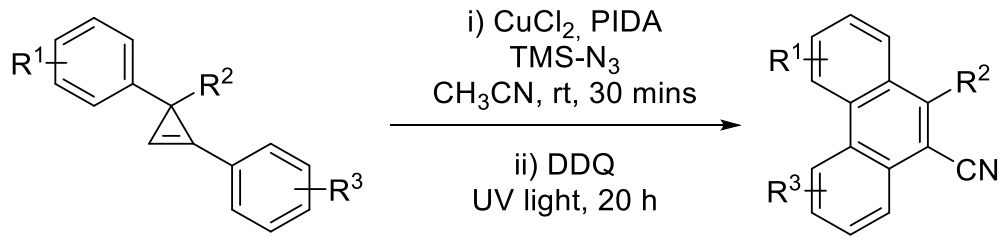

A $10 \mathrm{~mL}$ test tube was charged with $\mathrm{CuCl}_{2}(2 \mathrm{mg}, 0.05 \mathrm{mmol}, 0.05$ equiv. $)$, the cyclopropene ( 0.30 mmol, 1.0 equiv.) and PIDA (174 $\mathrm{mg}, 0.540 \mathrm{mmol}, 1.8$ equiv.). The tube was sealed, evacuated and back-filled with nitrogen (3 times). Then $\mathrm{CH}_{3} \mathrm{CN}(3 \mathrm{~mL}, 0.1 \mathrm{M}$ ) and TMS-N $3(72 \mu \mathrm{L}, 0.54$ mmol, 1.8 equiv.) were added via syringe and the resulting mixture was stirred at room temperature for 30 minutes. Then a solution of DDQ (commercial batch recrystallized from distilled $\mathrm{CHCl}_{3}, 75$ $\mathrm{mg}, 0.33$ mmol, 1.1 equiv.) in $\mathrm{CH}_{3} \mathrm{CN}$ (6 mL) was added to the reaction mixture before being placed under UV irradiation $(365 \mathrm{~nm})$ in a Rayonet reactor for 20 hours. The reaction mixture was then diluted with EtOAc and concentrated under reduced pressure. The obtained residue was filtered through a small pad of silica gel eluting with a 1:1 mixture of pentane:DCM and the filtrate was evaporated under reduced pressure. The obtained crude residue was then subjected to column chromatography using the indicated solvents.

\section{Methyl 10-cyanophenanthrene-9-carboxylate (20)}<smiles>CC(=O)c1c(C#N)c2ccccc2c2ccccc12</smiles>

20

Following the general procedure D, starting from methyl 1,2-diphenylcycloprop-2-enecarboxylate (39) $(75 \mathrm{mg}, 0.30 \mathrm{mmol}, 1.0$ equiv.), the title compound 20 was obtained after purification by column chromatography on Biotage (Büchi flashpure cartridge $25 \mathrm{~g}$, Pentane:EtOAc 90:10) as a white solid (63 mg, $0.24 \mathrm{mmol}, 80 \%$ yield). Characterization as described in section 5 .

\section{Tert-butyl 10-cyanophenanthrene-9-carboxylate (21)}

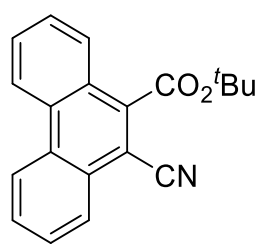

21

Following the general procedure D, starting from tert-butyl 1,2-diphenylcycloprop-2enecarboxylate (78) (88 $\mathrm{mg}, 0.30 \mathrm{mmol}, 1.0$ equiv.), the title compound 21 was obtained after 
purification by column chromatography on Biotage (Büchi flashpure cartridge $25 \mathrm{~g}$, Pentane:EtOAc 95:5) as a white solid (68 mg, $0.22 \mathrm{mmol}, 75 \%$ yield). $\mathbf{R}_{f} 0.32$ (Pentane:EtOAc 95:5); m.p = 125-126 ${ }^{\circ} \mathrm{C}$; ${ }^{1} \mathbf{H}$ NMR (400 MHz, Chloroform-d) $\delta 8.75-8.67$ (m, 2H, ArH), 8.41 $-8.34(\mathrm{~m}, 1 \mathrm{H}, \mathrm{Ar} H), 8.13(\mathrm{dd}, J=8.3,1.3 \mathrm{~Hz}, 1 \mathrm{H}, \mathrm{ArH}), 7.85-7.75$ (m, 3H, ArH), $7.75-7.69$ $(\mathrm{m}, 1 \mathrm{H}, \mathrm{ArH}), 1.78\left(\mathrm{~s}, 9 \mathrm{H}, \mathrm{CO}_{2} \mathrm{C}\left(\mathrm{CH}_{3}\right)_{3}\right) ;{ }^{13} \mathrm{C}$ NMR (101 MHz, Chloroform-d) $\delta$ 165.7, 139.2, $131.9,130.3,130.1,129.2,128.6,128.2$, 128.1, 127.1, 126.9, 126.9, 123.2, 123.1, 116.2, 108.1, 85.0, 28.2; IR $\widetilde{\boldsymbol{v}}_{\max } 3073(\mathrm{w}), 2980(\mathrm{~m}), 2933$ (m), $2113(\mathrm{w}), 1720$ (s), $1610(\mathrm{w}), 1526(\mathrm{w}), 1453$ (m), 1378 (s), 1295 (w), 1254 (s), 1215 (m), 1152 (s), 1058 (m), 959 (w), 910 (w), 841 (m), 756 (s), 729 (s), 625 (w); HRMS (APCI) calcd for $\mathrm{C}_{20} \mathrm{H}_{17} \mathrm{NNaO}_{2}{ }^{+}[\mathrm{M}+\mathrm{Na}]^{+}$326.1151; found 326.1152.

\section{0-propionylphenanthrene-9-carbonitrile (22)}

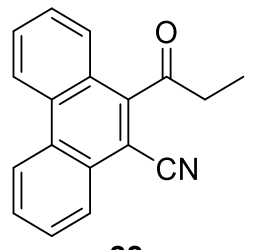

22

Following the general procedure D, starting from 1-(1,2-diphenylcycloprop-2-en-1-yl)propan-1one (79) (75 $\mathrm{mg}, 0.30 \mathrm{mmol}, 1.0$ equiv.), the title compound 22 was obtained after purification by column chromatography on Biotage (Büchi flashpure cartridge $25 \mathrm{~g}$, Pentane:EtOAc 95:5) as a colorless oil (40 mg, 0.15 mmol, $51 \%$ yield). $\mathbf{R}_{f} 0.27$ (Pentane:EtOAc 95:5); ${ }^{1} \mathbf{H}$ NMR (400 MHz, Chloroform- $d) \delta 8.82-8.71(\mathrm{~m}, 2 \mathrm{H}, \mathrm{ArH}), 8.41-8.34(\mathrm{~m}, 1 \mathrm{H}, \mathrm{ArH}), 7.90-7.79(\mathrm{~m}, 3 \mathrm{H}, \operatorname{Ar} H)$, $7.78-7.67(\mathrm{~m}, 2 \mathrm{H}, \mathrm{ArH}), 3.18\left(\mathrm{q}, J=7.2 \mathrm{~Hz}, 2 \mathrm{H}, \mathrm{CH}_{2}\right), 1.41\left(\mathrm{t}, J=7.2 \mathrm{~Hz}, 3 \mathrm{H}, \mathrm{CH}_{3}\right)$; ${ }^{13} \mathbf{C} \mathbf{N M R}$ (101 MHz, Chloroform-d) $\delta$ 206.5, 147.6, 132.1, 130.3, 130.0, 129.1, 128.8, 128.3, 128.0, 126.7, 126.6, 126.5, 123.6, 123.2, 116.3, 105.3, 38.4, 7.9; IR $\widetilde{\boldsymbol{v}}_{\max } 3389(\mathrm{w}), 3065$ (w), $2977(\mathrm{~m}), 2928$ (m), $2224(\mathrm{~m}), 2103(\mathrm{w}), 1706(\mathrm{~s}), 1606(\mathrm{w}), 1450(\mathrm{~m}), 1377(\mathrm{~m}), 1340(\mathrm{~m}), 1246(\mathrm{~m}), 1168(\mathrm{~m})$, $1128(\mathrm{~m}), 1047(\mathrm{~m}), 905(\mathrm{~m}), 852(\mathrm{~m}), 758(\mathrm{~s}), 731(\mathrm{~s})$; HRMS (ESI) calcd for $\mathrm{C}_{18} \mathrm{H}_{14} \mathrm{NO}^{+}[\mathrm{M}+\mathrm{H}]^{+}$ 260.1070; found 260.1077.

10-(Trifluoromethyl)phenanthrene-9-carbonitrile (23)

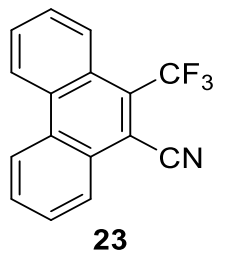

Following the general procedure D, starting from (1-(trifluoromethyl)cycloprop-2-ene-1,2diyl)dibenzene (80) (78 $\mathrm{mg}, 0.30 \mathrm{mmol}, 1.0$ equiv.), the title compound 23 was obtained after purification by column chromatography on Biotage (Büchi flashpure cartridge $25 \mathrm{~g}$, Pentane:EtOAc 95:5) as a white solid (53 mg, $0.20 \mathrm{mmol}, 65 \%$ yield). $\mathbf{R}_{\boldsymbol{f}}$ 0.29 (Pentane:EtOAc 95:5); m.p = 208-209 ${ }^{\circ} \mathrm{C}$; ${ }^{1} \mathbf{H}$ NMR (400 MHz, Chloroform- $d$ ) $\delta 8.78$ (ddd, $J=13.7,8.3,1.3 \mathrm{~Hz}$, $2 \mathrm{H}, \mathrm{Ar} H), 8.59(\mathrm{dd}, J=8.1,1.5 \mathrm{~Hz}, 1 \mathrm{H}, \mathrm{ArH}), 8.35(\mathrm{dt}, J=6.3,2.1 \mathrm{~Hz}, 1 \mathrm{H}, \mathrm{Ar} H), 7.94-7.82(\mathrm{~m}$, $3 \mathrm{H}, \mathrm{ArH}), 7.78$ (ddd, $J=8.4,7.0,1.3 \mathrm{~Hz}, 1 \mathrm{H}, \mathrm{Ar} H) ;{ }^{13} \mathrm{C}$ NMR (101 MHz, Chloroform-d) $\delta$ 132.2, 
$131.2,131.1(\mathrm{t}, J=29.9 \mathrm{~Hz}), 130.5,130.3,129.1,128.5,127.9,127.8,126.8(\mathrm{q}, J=4.1 \mathrm{~Hz}), 125.9$, 123.4, $123.3(\mathrm{q}, J=276.8 \mathrm{~Hz}), 123.1,114.9,109.6(\mathrm{q}, J=3.3 \mathrm{~Hz}) ;{ }^{19} \mathbf{F}$ NMR $(376 \mathbf{~ M H z}$, Chloroform- $\boldsymbol{d}) \delta-54.8\left(\mathrm{~d}, J=2.2 \mathrm{~Hz}\right.$ ); IR $\widetilde{\boldsymbol{v}}_{\max } 2928(\mathrm{~m}), 2853(\mathrm{~m}), 2360(\mathrm{w}), 2229(\mathrm{w}), 2113$ (m), $1740(\mathrm{~m}), 1528(\mathrm{~m}), 1453(\mathrm{~m}), 1373(\mathrm{~m}), 1314(\mathrm{~m}), 1249(\mathrm{~m}), 1164(\mathrm{~s}), 1123(\mathrm{~s}), 1024(\mathrm{~m})$, $766(\mathrm{~m}), 714(\mathrm{~m})$; HRMS (APPI) calcd for $\mathrm{C}_{16} \mathrm{H}_{8} \mathrm{~F}_{3} \mathrm{~N}^{+}[\mathrm{M}]^{+} 271.0603$; found 271.0610.

\section{Ethyl 6-bromo-10-cyanophenanthrene-9-carboxylate (24)}

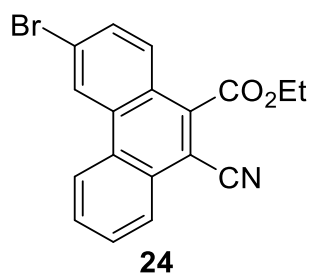

Following the general procedure D, starting from ethyl 1-(4-bromophenyl)-2-phenylcycloprop-2enecarboxylate (81) (103 $\mathrm{mg}, 0.300 \mathrm{mmol}, 1.0$ equiv.), the title compound 24 was obtained after purification by column chromatography on Biotage (Büchi flashpure cartridge $25 \mathrm{~g}$, Pentane:DCM 60:40) as a white solid (75 mg, $0.21 \mathrm{mmol}, 71 \%$ yield). $\mathbf{R}_{f} 0.42$ (Pentane:DCM 50:50); $\mathbf{m} . \mathbf{p}=159$ $160{ }^{\circ} \mathrm{C}$; ${ }^{1} \mathrm{H}$ NMR (400 MHz, Chloroform-d) $\delta 8.69(\mathrm{~d}, J=2.0 \mathrm{~Hz}, 1 \mathrm{H}, \mathrm{ArH}), 8.54-8.45$ (m, 1H, $\mathrm{ArH}), 8.35-8.26(\mathrm{~m}, 1 \mathrm{H}, \mathrm{ArH}), 7.95(\mathrm{~d}, J=8.9 \mathrm{~Hz}, 1 \mathrm{H}, \mathrm{ArH}), 7.83-7.68(\mathrm{~m}, 3 \mathrm{H}, \mathrm{ArH}), 4.66(\mathrm{q}$, $\left.J=7.2 \mathrm{~Hz}, 2 \mathrm{H}, \mathrm{CH}_{2} \mathrm{CH}_{3}\right), 1.56$ (t, $\left.J=7.2 \mathrm{~Hz}, 3 \mathrm{H}, \mathrm{CH}_{2} \mathrm{CH}_{3}\right) ;{ }^{13} \mathbf{C}$ NMR (101 MHz, Chloroformd) $\delta 165.9,137.1,132.9,131.4,129.7,129.3,129.1,128.6,128.2,127.1,126.0,125.3,125.2,123.1$, 115.7, 109.4, 63.2, 14.2; IR $\widetilde{\boldsymbol{v}}_{\max } 3668$ (w), $2981(\mathrm{~m}), 2361(\mathrm{w}), 2227(\mathrm{w}), 1727$ (s), 1593 (m), 1485 (m), 1449 (m), 1389 (m), 1319 (m), 1242 (s), 1209 (s), 1158 (m), 1065 (s), 1013 (s), 957 (w), 905 (w), 865 (m), $818(\mathrm{~m}), 758$ (s); HRMS (ESI) calcd for $\mathrm{C}_{18} \mathrm{H}_{12}{ }^{79} \mathrm{BrNNaO}_{2}{ }^{+}[\mathrm{M}+\mathrm{Na}]^{+} 375.9944$; found 375.9954 .

Methyl 10-cyano-6-methoxyphenanthrene-9-carboxylate (25)

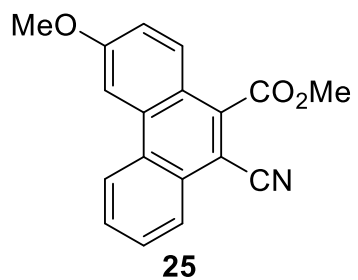

Following the general procedure D, starting from methyl 1-(4-methoxyphenyl)-2phenylcycloprop-2-enecarboxylate (82) (84 mg, $0.30 \mathrm{mmol}, 1.0$ equiv.), the title compound 25 was obtained after purification by column chromatography on Biotage (Büchi flashpure cartridge $25 \mathrm{~g}$, Pentane:EtOAc 95:5) as a white solid (61 mg, $0.21 \mathrm{mmol}, 70 \%$ yield). $\mathbf{R}_{f} 0.34$ (Pentane:EtOAc 90:10); m.p = 163-164 ${ }^{\circ} \mathrm{C}$; ${ }^{1} \mathbf{H}$ NMR (400 MHz, Chloroform-d) $\delta 8.67-8.59(\mathrm{~m}, 1 \mathrm{H}, \mathrm{ArH}), 8.41$ - 8.33 (m, 1H, ArH), $8.10-8.00$ (m, 2H, ArH), $7.83-7.74(\mathrm{~m}, 2 \mathrm{H}, \mathrm{ArH}), 7.33$ (dd, J=9.2, 2.5 $\mathrm{Hz}, 1 \mathrm{H}, \mathrm{ArH}), 4.17$ (s, 3H, $\left.\mathrm{CO}_{2} \mathrm{CH}_{3}\right), 4.06$ (s, 3H, $\left.\mathrm{OCH}_{3}\right) ;{ }^{13} \mathrm{C}$ NMR (101 MHz, Chloroform-d) $\delta$ 167.1, 161.1, 137.6, 134.0, 129.9, 129.0, 128.9, 128.6, 127.1, 123.2, 121.4, 118.5, 116.5, 106.4, 104.5, 55.8, 53.5; IR $\widetilde{\boldsymbol{v}}_{\max } 3010(\mathrm{w}), 2952(\mathrm{w}), 2846(\mathrm{w}), 2358(\mathrm{~m}), 2224(\mathrm{~m}), 2052(\mathrm{w}), 1729(\mathrm{~s})$, 1616 (s), 1515 (m), 1446 (m), 1386 (m), 1325 (m), 1241 (s), 1155 (m), 1065 (m), 1025 (m), 988 
(m), $912(\mathrm{~m}), 835(\mathrm{~m}), 767(\mathrm{~m}), 736(\mathrm{~m}), 669(\mathrm{~m}), 627(\mathrm{~m})$; HRMS (ESI) calcd for $\mathrm{C}_{18} \mathrm{H}_{14} \mathrm{NO}_{3}{ }^{+}$ $[\mathrm{M}+\mathrm{H}]^{+}$292.0968; found 292.0968.

Methyl 3-(tert-butyl)-10-cyano-6-methylphenanthrene-9-carboxylate (26)<smiles>CC(=O)c1c(C#N)c2ccc(C(C)(C)C)cc2c2ccc(C)cc12</smiles>

Following the general procedure D, starting from methyl 2-(4-(tert-butyl)phenyl)-1-(ptolyl)cycloprop-2-enecarboxylate (83) $(96 \mathrm{mg}, 0.30 \mathrm{mmol}, 1.0$ equiv.), the title compound 26 was obtained after purification by column chromatography on Biotage (Büchi flashpure cartridge $25 \mathrm{~g}$, Pentane:DCM 60:40) as a white solid ( $80 \mathrm{mg}, 0.24 \mathrm{mmol}, 80 \%$ yield). $\mathbf{R}_{f} 0.47$ (Pentane:DCM 50:50); m.p = 184-185 ${ }^{\circ} \mathrm{C}$; ${ }^{1} \mathbf{H}$ NMR (400 MHz, Chloroform-d) $\delta 8.67$ (d, $J=1.9 \mathrm{~Hz}, 1 \mathrm{H}, \operatorname{Ar} H$ ), 8.52 (s, 1H, ArH), 8.32 (d, $J=8.6 \mathrm{~Hz}, 1 \mathrm{H}, \operatorname{ArH}), 8.02$ (d, $J=8.4 \mathrm{~Hz}, 1 \mathrm{H}, \operatorname{ArH}), 7.85$ (dd, $J=8.7$, $1.9 \mathrm{~Hz}, 1 \mathrm{H}, \mathrm{ArH}), 7.52$ (dd, $J=8.5,1.6 \mathrm{~Hz}, 1 \mathrm{H}, \mathrm{ArH}), 4.16\left(\mathrm{~s}, 3 \mathrm{H}, \mathrm{CO}_{2} \mathrm{CH}_{3}\right), 2.68\left(\mathrm{~s}, 3 \mathrm{H}, \mathrm{CH}_{3}\right)$, $1.52\left(\mathrm{~s}, 9 \mathrm{H},{ }^{t} \mathrm{Bu}\right) ;{ }^{13} \mathbf{C}$ NMR (101 MHz, Chloroform-d) $\delta 167.2,152.6,140.5,136.8,132.1,130.0$, $129.9,127.1,127.1,126.8,126.2,125.0,122.8,118.7,116.5,107.9,53.4,35.7,31.5,22.5$; IR $\widetilde{v}_{\max }$ $2961(\mathrm{w}), 2872(\mathrm{w}), 2255$ (w), 2226 (w), $1731(\mathrm{~m}), 1616(\mathrm{w}), 1509(\mathrm{w}), 1444(\mathrm{w}), 1378(\mathrm{w}), 1314$ (w), 1258 (m), 1221 (m), 1168 (m), 1073 (w), 988 (w), 909 (s), 732 (s), 650 (w); HRMS (ESI) calcd for $\mathrm{C}_{22} \mathrm{H}_{22} \mathrm{NO}_{2}{ }^{+}[\mathrm{M}+\mathrm{H}]^{+} 332.1645$; found 332.1650.

\section{Methyl 10-cyano-6-methyl-3-(trifluoromethoxy)phenanthrene-9-carboxylate (27)}<smiles>CCOc1ccc2c(C#N)c(C(C)=O)c3ccc(C)cc3c2c1</smiles>

Following the general procedure D, starting from methyl 1-(p-tolyl)-2-(4(trifluoromethoxy)phenyl)cycloprop-2-enecarboxylate (84) (104 mg, $0.300 \mathrm{mmol}, 1.0$ equiv.), the title compound 27 was obtained after purification by column chromatography on Biotage (Büchi flashpure cartridge $25 \mathrm{~g}$, Pentane:EtOAc 95:5) as a white solid (82 mg, $0.23 \mathrm{mmol}, 76 \%$ yield). $\mathbf{R}_{f}$ 0.42 (Pentane:EtOAc 90:10); m.p = 197-198 ${ }^{\circ} \mathrm{C}$; ${ }^{\mathbf{1}} \mathbf{H}$ NMR (400 MHz, Chloroform- $\left.\boldsymbol{d}\right) \delta 8.44-$ 8.39 (m, 1H, ArH), 8.36 (d, J=9.0 Hz, 1H, ArH), 8.30 (s, 1H, ArH), 7.97 (d, J= 8.5 Hz, 1H, ArH), $7.60(\mathrm{ddd}, J=8.9,2.4,1.1 \mathrm{~Hz}, 1 \mathrm{H}, \mathrm{ArH}), 7.53(\mathrm{dd}, J=8.5,1.7 \mathrm{~Hz}, 1 \mathrm{H}, \mathrm{ArH}), 4.18\left(\mathrm{~s}, 3 \mathrm{H}, \mathrm{CO}_{2} \mathrm{CH}_{3}\right)$, 2.65 (s, 3H, CH $) ;{ }^{13} \mathbf{C}$ NMR (101 MHz, Chloroform-d) $\delta 166.6,149.6(\mathrm{~d}, J=2.3 \mathrm{~Hz}), 141.3$, 138.0, 131.4, 131.1, 130.8, 129.2, 127.2, 126.4, 125.0, 123.0, 121.7, 120.7 (q, $J=258.6 \mathrm{~Hz}), 115.9$, 114.7, 107.4, 53.6, 22.4; ${ }^{19}$ F NMR (376 MHz, Chloroform-d) $\delta$-57.5; IR $\widetilde{\boldsymbol{v}}_{\max } 3087$ (w), 2921 (w), $2227(\mathrm{w}), 1742(\mathrm{~m}), 1618(\mathrm{w}), 1513(\mathrm{w}), 1442(\mathrm{w}), 1378(\mathrm{w}), 1249$ (s), 1206 (s), 1161 (s), $1069(\mathrm{w}), 976(\mathrm{w}), 908(\mathrm{~m}), 867(\mathrm{~m}), 809(\mathrm{w}), 734$ (s), 648 (w); HRMS (ESI) calcd for $\mathrm{C}_{19} \mathrm{H}_{12} \mathrm{~F}_{3} \mathrm{NNaO}_{3}{ }^{+}[\mathrm{M}+\mathrm{Na}]^{+} 382.0661$; found 382.0654 . 
Methyl 1-chloro-10-cyanophenanthrene-9-carboxylate (28)

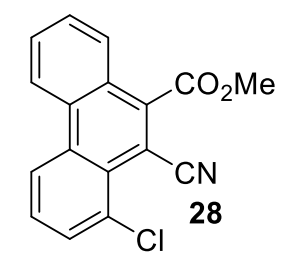

Following the general procedure D, starting from methyl 2-(2-chlorophenyl)-1-phenylcycloprop2-enecarboxylate (85) (85 $\mathrm{mg}, 0.30 \mathrm{mmol}, 1.0$ equiv.), the title compound 28 was obtained after purification by column chromatography on Biotage (Büchi flashpure cartridge $25 \mathrm{~g}$, Pentane:EtOAc 95:5 to 90:10) as a grey solid (57 mg, 0.19 mmol, $61 \%$ yield, 95\% purity). ${ }^{29} \mathbf{R}_{f}$ 0.37 (Pentane:EtOAc 80:20); m.p = $171-172{ }^{\circ} \mathrm{C} ; \mathbf{1}^{\mathbf{1}} \mathbf{H}$ NMR (400 MHz, Chloroform- $\left.\boldsymbol{d}\right) \delta 8.72-$ $8.64(\mathrm{~m}, 2 \mathrm{H}, \mathrm{ArH}), 7.89-7.82(\mathrm{~m}, 2 \mathrm{H}, \mathrm{ArH}), 7.81-7.65$ (m, 3H, $\mathrm{ArH}), 4.19\left(\mathrm{~s}, 3 \mathrm{H}, \mathrm{CO}_{2} \mathrm{CH}_{3}\right)$; ${ }^{13}$ C NMR (101 MHz, Chloroform-d) $\delta$ 167.5, 144.3, 132.7, 132.4, 131.6, 131.1, 131.0, 129.0, 128.9, 127.0, 126.5, 124.9, 123.7, 122.4, 117.4, 104.9, 54.0; IR $\widetilde{\boldsymbol{v}}_{\max } 3072(\mathrm{w}), 2951(\mathrm{~m}), 2925$ (m), $2221(\mathrm{w}), 2109(\mathrm{w}), 1736(\mathrm{~s}), 1603(\mathrm{w}), 1521(\mathrm{~m}), 1442$ (s), $1375(\mathrm{~m}), 1248$ (s), $1213(\mathrm{~s}), 1165$ (m), $1127(\mathrm{~m}), 1051(\mathrm{~m}), 985(\mathrm{~m}), 918$ (m), $799(\mathrm{~m}), 756$ (s), 690 (m), 642 (m); HRMS (ESI) calcd for $\mathrm{C}_{17} \mathrm{H}_{11} \mathrm{ClNO}_{2}{ }^{+}[\mathrm{M}+\mathrm{H}]^{+} 296.0473$; found 296.0476.

\section{Methyl 10-cyano-8-fluorophenanthrene-9-carboxylate (29)}

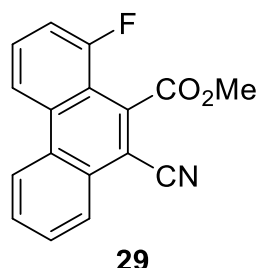

Following the general procedure D, starting from methyl 1-(2-fluorophenyl)-2-phenylcycloprop2-enecarboxylate (86) (80 $\mathrm{mg}, 0.30 \mathrm{mmol}, 1.0$ equiv.), the title compound 29 was obtained after purification by column chromatography on Biotage (Büchi flashpure cartridge $25 \mathrm{~g}$, Pentane:EtOAc 90:10) as a white solid (62 mg, $0.22 \mathrm{mmol}, 70 \%$ yield, 95\% purity). ${ }^{29} \mathbf{R}_{f} 0.27$ (Pentane:EtOAc 90:10); m.p = 171-172 ${ }^{\circ} \mathrm{C} ; \mathbf{1}^{\mathbf{H}} \mathbf{H}$ NMR (400 MHz, Chloroform-d) $\delta 8.59-8.51$ $(\mathrm{m}, 1 \mathrm{H}, \operatorname{Ar} H), 8.40(\mathrm{~d}, J=8.4 \mathrm{~Hz}, 1 \mathrm{H}, \operatorname{Ar} H), 8.26-8.19(\mathrm{~m}, 1 \mathrm{H}, \mathrm{ArH}), 7.79-7.68(\mathrm{~m}, 3 \mathrm{H}, \operatorname{Ar} H)$, 7.31 (ddd, $J=11.4,7.9,1.0 \mathrm{~Hz}, 1 \mathrm{H}, \mathrm{ArH}), 4.14\left(\mathrm{~s}, 3 \mathrm{H}, \mathrm{CO}_{2} \mathrm{CH}_{3}\right) ;{ }^{13} \mathrm{C}$ NMR (101 MHz, Chloroform- $d) \delta 167.6,158.5(\mathrm{~d}, J=255.2 \mathrm{~Hz}), 134.3,133.4(\mathrm{~d}, J=2.5 \mathrm{~Hz}), 130.8(\mathrm{~d}, J=9.5$ $\mathrm{Hz}), 129.7,129.3,129.2(\mathrm{~d}, J=2.6 \mathrm{~Hz}), 127.8,126.7,123.5,119.2$ (d, $J=3.8 \mathrm{~Hz}), 116.5(\mathrm{~d}, J=$ 12.5 Hz), 115.5, $113.4(\mathrm{~d}, J=21.5 \mathrm{~Hz}), 109.0,53.8 ;{ }^{19}$ F NMR (376 MHz, Chloroform- $d$ ) $\delta-110.8$ (dd, $J=11.3,5.7 \mathrm{~Hz}$ ); IR $\widetilde{\boldsymbol{v}}_{\max } 3068(\mathrm{w}), 2952(\mathrm{w}), 2854$ (w), 2226 (w), 1739 (s), 1620 (w), 1579 (w), 1522 (w), 1453 (s), 1371 (m), 1325 (w), 1249 (s), 1207 (s), 1158 (m), 1067 (m), 987 (m), 917 (m), 751 (s); HRMS (ESI) calcd for $\mathrm{C}_{17} \mathrm{H}_{11} \mathrm{FNO}_{2}{ }^{+}[\mathrm{M}+\mathrm{H}]^{+}$280.0768; found 280.0774.

Methyl 8-chloro-10-cyano-6-fluoro-3-methylphenanthrene-9-carboxylate (30) 


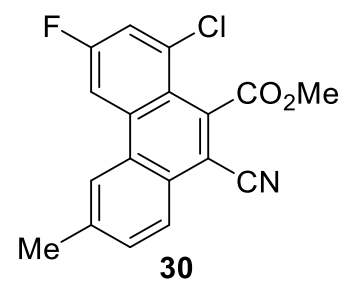

Following the general procedure $\mathrm{D}$, starting from methyl 1-(2-chloro-4-fluorophenyl)-2-(ptolyl)cycloprop-2-enecarboxylate (87) (95 $\mathrm{mg}, 0.30 \mathrm{mmol}, 1.0$ equiv.), the title compound $\mathbf{3 0}$ was obtained after purification by column chromatography on Biotage (Büchi flashpure cartridge $25 \mathrm{~g}$, Pentane:DCM 60:40) as a white solid (57 mg, $0.17 \mathrm{mmol}, 55 \%$ yield, $95 \%$ purity) ${ }^{29} \mathbf{R}_{f} 0.42$ (Pentane:DCM 50:50); m.p = $185-186{ }^{\circ} \mathrm{C} ;{ }^{1} \mathbf{H}$ NMR (400 MHz, Chloroform- $\left.\boldsymbol{d}\right) \delta 8.25-8.18(\mathrm{~m}$, $2 \mathrm{H}, \operatorname{Ar} H), 8.13(\mathrm{~d}, J=8.3 \mathrm{~Hz}, 1 \mathrm{H}, \mathrm{Ar} H), 7.62-7.55(\mathrm{~m}, 1 \mathrm{H}, \mathrm{Ar} H), 7.46(\mathrm{dd}, J=7.8,2.5 \mathrm{~Hz}, 1 \mathrm{H}$, $\mathrm{ArH}$ ), 4.05 (s, 3H, $\left.\mathrm{CO}_{2} \mathrm{CH}_{3}\right), 2.59$ (s, 3H, $\left.\mathrm{CH}_{3}\right) ;{ }^{13} \mathrm{C}$ NMR (101 MHz, Chloroform-d) $\delta 167.9$, $162.0(\mathrm{~d}, J=254.2 \mathrm{~Hz}), 140.5,135.5(\mathrm{~d}, J=1.8 \mathrm{~Hz}), 135.3(\mathrm{~d}, J=9.2 \mathrm{~Hz}), 133.7(\mathrm{~d}, J=11.2 \mathrm{~Hz})$, $131.9,129.5(\mathrm{~d}, J=4.4 \mathrm{~Hz}), 126.8,126.0,123.4,121.6(\mathrm{~d}, J=2.3 \mathrm{~Hz}), 119.7(\mathrm{~d}, J=26.9 \mathrm{~Hz})$, $115.7,110.5$ (d, $J=2.9 \mathrm{~Hz}), 108.1$ (d, $J=22.4 \mathrm{~Hz}), 53.9,22.4$; ${ }^{\mathbf{1 9}}$ F NMR (376 MHz, Chloroformd) $\delta-106.9(\mathrm{dd}, J=9.9,7.8 \mathrm{~Hz})$; IR $\widetilde{\boldsymbol{v}}_{\max } 2955(\mathrm{~m}), 2928(\mathrm{~m}), 2359(\mathrm{~m}), 2226(\mathrm{~m}), 2054(\mathrm{~m}), 1734$ (s), $1612(\mathrm{~s}), 1577(\mathrm{~m}), 1508(\mathrm{~m}), 1437(\mathrm{~m}), 1374(\mathrm{~m}), 1314(\mathrm{~m}), 1236$ (s), $1195(\mathrm{~s}), 1177(\mathrm{~s}), 1130$ (m), $1070(\mathrm{~m}), 986(\mathrm{~m}), 908(\mathrm{~m}), 863(\mathrm{~s}), 820(\mathrm{~m}), 775(\mathrm{~m}), 739(\mathrm{~m}), 634(\mathrm{~m})$; HRMS (ESI) calcd for $\mathrm{C}_{18} \mathrm{H}_{12} \mathrm{ClFNO}_{2}{ }^{+}[\mathrm{M}+\mathrm{H}]^{+}$328.0535; found 328.0536 .

\section{Methyl 10-cyano-2,4-bis(trifluoromethyl)phenanthrene-9-carboxylate (31)}<smiles></smiles>

Following the general procedure D, starting from methyl 2-(3,5-bis(trifluoromethyl)phenyl)-1phenylcycloprop-2-enecarboxylate (88) (116 $\mathrm{mg}, 0.300 \mathrm{mmol}, 1.0$ equiv.), the title compound 31 was obtained after purification by column chromatography on Biotage (Büchi flashpure cartridge $25 \mathrm{~g}$, Pentane:EtOAc 90:10) as a white solid $\left(81 \mathrm{mg}, 0.20 \mathrm{mmol}, 68 \%\right.$ yield). $\mathbf{R}_{f} 0.35$ (Pentane:EtOAc 90:10); m.p = 177-178 ${ }^{\circ} \mathrm{C} ;{ }^{1} \mathbf{H}$ NMR (400 MHz, Chloroform-d) $\delta 8.87(\mathrm{~s}, 1 \mathrm{H}$, $\operatorname{ArH}), 8.84-8.77(\mathrm{~m}, 1 \mathrm{H}, \operatorname{Ar} H), 8.44$ (s, 1H, $\operatorname{ArH}), 8.17-8.09$ (m, 1H, $\operatorname{ArH}), 7.92-7.84$ (m, 2H, $\mathrm{Ar} H$ ), 4.21 (s, $\left.3 \mathrm{H}, \mathrm{CO}_{2} \mathrm{CH}_{3}\right) ;{ }^{13} \mathbf{C}$ NMR (101 MHz, Chloroform-d) $\delta$ 166.0, 140.8, 131.5, 130.4, 130.3, 130.0, 129.9-129.7 (m), 129.6 - 129.2 (m), 128.8, 128.7, 128.6-128.2 (m), 128.0, 127.0, $126.8-126.3(\mathrm{~m}), 123.1(\mathrm{q}, J=272.7 \mathrm{~Hz}), 123.0(\mathrm{q}, J=273.4 \mathrm{~Hz}), 115.1,108.7,54.0 ;{ }^{19} \mathbf{F}$ NMR (376 MHz, Chloroform- $\boldsymbol{d}$ ) $\delta$-54.6, -62.6; IR $\widetilde{\boldsymbol{v}}_{\max } 2959$ (w), 1739 (m), $1632(\mathrm{w}), 1586(\mathrm{w}), 1532$ (m), $1443(\mathrm{~m}), 1347(\mathrm{~m}), 1272(\mathrm{~s}), 1171(\mathrm{~s}), 1131(\mathrm{~s}), 1005(\mathrm{~m}), 911(\mathrm{~s}), 861(\mathrm{~m}), 777(\mathrm{~m}), 732(\mathrm{~s})$, $689(\mathrm{~m}), 672(\mathrm{~m})$; HRMS (ESI) calcd for $\mathrm{C}_{19} \mathrm{H}_{9} \mathrm{~F}_{6} \mathrm{NNaO}_{2}{ }^{+}[\mathrm{M}+\mathrm{Na}]^{+} 420.0430$; found 420.0439 .

\section{Methyl 5-cyanonaphtho[1,2-b]thiophene-4-carboxylate (32)}




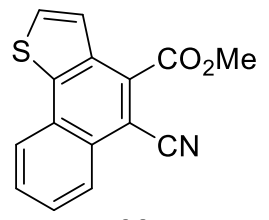

32

Following the general procedure D, starting from methyl 2-phenyl-1-(thiophen-3-yl)cycloprop-2enecarboxylate (89) (77 $\mathrm{mg}, 0.30 \mathrm{mmol}, 1.0$ equiv.), the title compound 32 was obtained after purification by column chromatography on Biotage (Büchi flashpure cartridge $25 \mathrm{~g}$, Pentane:EtOAc 95:5) as a white solid (49 mg, $0.18 \mathrm{mmol}, 61 \%$ yield). $\mathbf{R}_{f} 0.20$ (Pentane:EtOAc 95:5); m.p = 166-167 ${ }^{\circ} \mathrm{C}$; ${ }^{1} \mathbf{H}$ NMR (400 MHz, Chloroform-d) $\delta 8.49-8.43$ (m, $\left.1 \mathrm{H}, \mathrm{ArH}\right), 8.22$ $-8.15(\mathrm{~m}, 1 \mathrm{H}, \operatorname{ArH}), 7.96(\mathrm{~d}, J=5.5 \mathrm{~Hz}, 1 \mathrm{H}, \operatorname{HetArH}), 7.78-7.70(\mathrm{~m}, 2 \mathrm{H}, \operatorname{Ar} H), 7.66(\mathrm{~d}, J=5.6$ $\mathrm{Hz}, 1 \mathrm{H}, \mathrm{HetArH}), 4.15$ (s, 3H, $\left.\mathrm{CO}_{2} \mathrm{CH}_{3}\right) ;{ }^{13} \mathrm{C}$ NMR (101 MHz, Chloroform-d) $\delta 165.8,143.2$, 134.0, 130.6, 129.9, 129.7, 128.6, 128.5, 127.7, 127.4, 125.8, 124.2, 116.5, 109.0, 53.3; IR $\widetilde{\boldsymbol{v}}_{\max }$ 3097 (w), 2952 (w), 2849 (w), 2224 (w), 1724 (s), 1619 (w), 1552 (w), 1440 (m), 1308 (m), 1269 (m), 1223 (s), 1157 (m), 1096 (m), 975 (m), 896 (w), 804 (m), 762 (s), 662 (m); HRMS (ESI) calcd for $\mathrm{C}_{15} \mathrm{H}_{10} \mathrm{NO}_{2} \mathrm{~S}^{+}[\mathrm{M}+\mathrm{H}]^{+}$268.0427; found 268.0427.

\section{Methyl 6-cyanobenzo[c]phenanthrene-5-carboxylate (33)}

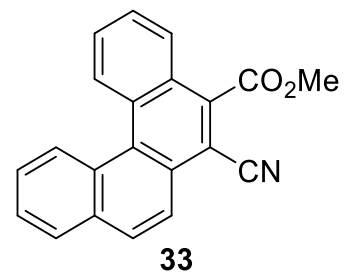

Following the general procedure D, starting from methyl 2-(naphthalen-2-yl)-1-phenylcycloprop2-enecarboxylate (90) (90 $\mathrm{mg}, 0.30 \mathrm{mmol}, 1.0$ equiv.), the title compound 33 was obtained after purification by column chromatography on Biotage (Büchi flashpure cartridge $25 \mathrm{~g}$, Pentane:EtOAc 95:5) as a yellow solid ( $71 \mathrm{mg}, 0.23 \mathrm{mmol}, 76 \%$ yield). $\mathbf{R}_{\boldsymbol{f}} 0.22$ (Pentane:EtOAc 95:5); m.p = 134-135 ${ }^{\circ} \mathrm{C}$; ${ }^{1} \mathbf{H}$ NMR (400 MHz, Chloroform-d) $\delta 9.04(\mathrm{~d}, J=8.6 \mathrm{~Hz}, 1 \mathrm{H}, \operatorname{Ar} H)$, $8.98-8.91(\mathrm{~m}, 1 \mathrm{H}, \operatorname{Ar} H), 8.29$ (d, $J=8.8 \mathrm{~Hz}, 1 \mathrm{H}, \operatorname{Ar} H), 8.24(\mathrm{dd}, J=8.3,1.4 \mathrm{~Hz}, 1 \mathrm{H}, \operatorname{Ar} H), 8.09$ - $8.02(\mathrm{~m}, 2 \mathrm{H}, \mathrm{Ar} H), 7.82(\mathrm{ddd}, J=8.5,6.9,1.4 \mathrm{~Hz}, 1 \mathrm{H}, \mathrm{ArH}), 7.77-7.69$ (m, 3H, ArH), 4.21 (s, $\left.3 \mathrm{H}, \mathrm{CO}_{2} \mathrm{CH}_{3}\right) ;{ }^{13} \mathbf{C} \mathbf{N M R}(\mathbf{1 0 1}$ MHz, Chloroform-d) $\delta$ 167.0, 136.7, 134.2, 131.7, 129.9, 129.4, 129.3, 129.3, 128.9, 128.7, 128.4, 128.3, 127.8, 127.8, 127.5, 127.3, 126.7, 123.3, 116.4, 108.8, 53.6; IR $\widetilde{\boldsymbol{v}}_{\max } 3058(\mathrm{w}), 2953(\mathrm{w}), 2849$ (w), 2225 (m), $2111(\mathrm{w}), 1728(\mathrm{~s}), 1610(\mathrm{w}), 1573(\mathrm{w})$, 1496 (m), 1430 (m), 1393 (m), 1261 (s), 1223 (s), 1151 (m), 1005 (m), $958(\mathrm{~m}), 905$ (m), $824(\mathrm{~s})$, 743 (s), 662 (m); HRMS (ESI) calcd for $\mathrm{C}_{21} \mathrm{H}_{14} \mathrm{NO}_{2}{ }^{+}[\mathrm{M}+\mathrm{H}]^{+} 312.1019$; found 312.1012.

Methyl 6-cyano-2-methoxybenzo[c]phenanthrene-5-carboxylate (34) 


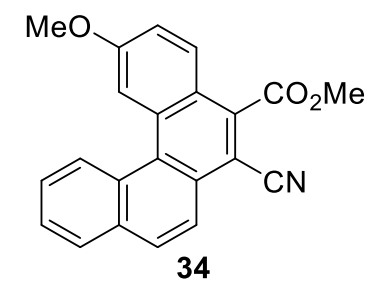

Following the general procedure D, starting from methyl 1-(4-methoxyphenyl)-2-(naphthalen-2yl)cycloprop-2-enecarboxylate (91) $(99 \mathrm{mg}, 0.30 \mathrm{mmol}, 1.0$ equiv.), the title compound 34 was obtained after purification by column chromatography on Biotage (Büchi flashpure cartridge $25 \mathrm{~g}$, Pentane:EtOAc 95:5) as a white solid (76 $\mathrm{mg}, 0.22 \mathrm{mmol}, 74 \%$ yield). $\mathbf{R}_{f} 0.33$ (Pentane:EtOAc 90:10); m.p = 166-167 ${ }^{\circ} \mathrm{C} ;{ }^{\mathbf{1}} \mathbf{H}$ NMR (400 MHz, Chloroform- $\left.\boldsymbol{d}\right) \delta 9.12-9.03(\mathrm{~m}, 1 \mathrm{H}, \mathrm{Ar} H), 8.51$ $(\mathrm{d}, J=2.5 \mathrm{~Hz}, 1 \mathrm{H}, \operatorname{Ar} H), 8.29(\mathrm{~d}, J=8.8 \mathrm{~Hz}, 1 \mathrm{H}, \operatorname{Ar} H), 8.19(\mathrm{~d}, J=9.2 \mathrm{~Hz}, 1 \mathrm{H}, \operatorname{Ar} H), 8.11-8.02$ $(\mathrm{m}, 2 \mathrm{H}, \mathrm{Ar} H), 7.75-7.67(\mathrm{~m}, 2 \mathrm{H} . \mathrm{Ar} H), 7.38(\mathrm{dd}, J=9.2,2.5 \mathrm{~Hz}, 1 \mathrm{H}, \mathrm{Ar} H), 4.19\left(\mathrm{~s}, 3 \mathrm{H}, \mathrm{CO}_{2} \mathrm{CH}_{3}\right)$, 4.03 (s, 3H, OCH $\left.{ }_{3}\right) ;{ }^{13} \mathbf{C}$ NMR (101 MHz, Chloroform-d) $\delta$ 167.1, 160.7, 136.5, 134.1, 133.8, 130.0, 129.7, 129.1, 128.5, 128.4, 128.3, 127.6, 127.5, 127.1, 123.6, 123.4, 118.4, 116.8, 109.8, 106.2, 55.8, 53.5; IR $\widetilde{\boldsymbol{v}}_{\max } 3052(\mathrm{w}), 3015(\mathrm{w}), 2952(\mathrm{~m}), 2843(\mathrm{w}), 2359(\mathrm{~m}), 2224(\mathrm{~m}), 2051(\mathrm{~m})$, $1730(\mathrm{~s}), 1614(\mathrm{~m}), 1512(\mathrm{~m}), 1439(\mathrm{~m}), 1407(\mathrm{~m}), 1334(\mathrm{~m}), 1232(\mathrm{~s}), 1150(\mathrm{~m}), 1111(\mathrm{~m}), 1038$ (m), $911(\mathrm{~m}), 821$ (s), 735 (s), $660(\mathrm{~m})$; HRMS (ESI) calcd for $\mathrm{C}_{22} \mathrm{H}_{16} \mathrm{NO}_{3}{ }^{+}[\mathrm{M}+\mathrm{H}]^{+}$342.1125; found 342.1132 .

\section{Ethyl 2-bromo-5-cyanobenzo[c]phenanthrene-6-carboxylate (35)}<smiles>CCOc1c(C#N)c(C)cc2ccc(Br)cc12</smiles>

Following the general procedure D, starting from ethyl 2-(4-bromophenyl)-1-(naphthalen-2yl)cycloprop-2-enecarboxylate (92) (118 $\mathrm{mg}, 0.300 \mathrm{mmol}, 1.0$ equiv.), after UV irradiation the crude mixture was directly filtered in order to recover the formed precipitate. The filter cake was washed with cold acetonitrile and pentane, and dried under reduced pressure to afford $\mathbf{3 5}(85 \mathrm{mg}$, $0.21 \mathrm{mmol}, 70 \%$ yield) as a grey powder, without further purification. $\mathbf{R}_{f} 0.25$ (Pentane:EtOAc 90:10); m.p = 202-203 ${ }^{\circ} \mathrm{C} ;{ }^{1} \mathbf{H}$ NMR (400 MHz, Chloroform-d) $\delta 9.21(\mathrm{~d}, J=1.9 \mathrm{~Hz}, 1 \mathrm{H}, \operatorname{Ar} H)$, $8.96-8.88(\mathrm{~m}, 1 \mathrm{H}, \operatorname{Ar} H), 8.38(\mathrm{~d}, J=8.8 \mathrm{~Hz}, 1 \mathrm{H}, \operatorname{Ar} H), 8.09-8.04(\mathrm{~m}, 1 \mathrm{H}, \operatorname{Ar} H), 8.04-7.97$ $(\mathrm{m}, 2 \mathrm{H}, \operatorname{Ar} H), 7.91(\mathrm{dd}, J=8.8,1.9 \mathrm{~Hz}, 1 \mathrm{H}, \operatorname{Ar} H), 7.82-7.73(\mathrm{~m}, 2 \mathrm{H}, \operatorname{Ar} H), 4.68(\mathrm{q}, J=7.2 \mathrm{~Hz}$, $\left.2 \mathrm{H}, \mathrm{CH}_{2} \mathrm{CH}_{3}\right), 1.56\left(\mathrm{t}, J=7.2 \mathrm{~Hz}, 3 \mathrm{H}, \mathrm{CH}_{2} \mathrm{CH}_{3}\right) ;{ }^{13} \mathrm{C}$ NMR (101 MHz, Chloroform-d) $\delta 166.5$, 137.4, 134.5, 131.6, 131.4, 131.1, 129.9, 129.9, 129.2, 128.9, 128.5, 128.5, 128.3, 128.1, 127.8, 126.6, 123.6, 122.9, 115.8, 108.1, 63.3, 14.2; IR $\widetilde{\boldsymbol{v}}_{\max } 2990(\mathrm{~m}), 1731(\mathrm{~s}), 1599(\mathrm{~m}), 1491(\mathrm{~m})$, $1457(\mathrm{~m}), 1397(\mathrm{~s}), 1359(\mathrm{~m}), 1298(\mathrm{~m}), 1266(\mathrm{~s}), 1220(\mathrm{~s}), 1154(\mathrm{~s}), 1076(\mathrm{~s}), 1012(\mathrm{~m}), 913(\mathrm{~s})$, $852(\mathrm{~m}), 737(\mathrm{~s}), 660(\mathrm{~m})$; HRMS (APPI) calcd for $\mathrm{C}_{22} \mathrm{H}_{15}{ }^{79} \mathrm{BrNO}_{2}{ }^{+}[\mathrm{M}+\mathrm{H}]^{+} 404.0281$; found 404.0295 .

\section{Methyl 5-cyanobenzo[g]chrysene-6-carboxylate (36)}




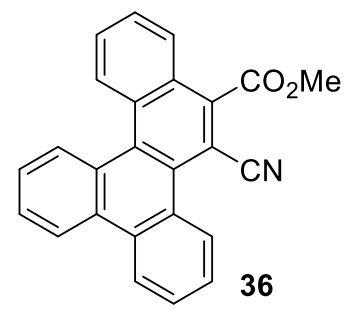

Following the general procedure $\mathrm{D}$, starting from methyl 2-(phenanthren-9-yl)-1-phenylcycloprop2-enecarboxylate (93) (105 mg, $0.300 \mathrm{mmol}, 1.0$ equiv.), the title compound 36 was obtained after purification by column chromatography on Biotage (Büchi flashpure cartridge $25 \mathrm{~g}$, Pentane:DCM 60:40) as a yellow solid (78 $\mathrm{mg}, 0.22 \mathrm{mmol}, 72 \%$ yield). $\mathbf{R}_{f} 0.35$ (Pentane:DCM 50:50); $\mathbf{m} . \mathbf{p}=$ $163-164{ }^{\circ} \mathrm{C} ;{ }^{1} \mathbf{H}$ NMR (400 MHz, Chloroform- $d$ ) $\delta 9.21-9.14(\mathrm{~m}, 1 \mathrm{H}, \mathrm{ArH}), 8.70-8.64(\mathrm{~m}, 1 \mathrm{H}$, $\operatorname{Ar} H), 8.63-8.56(\mathrm{~m}, 2 \mathrm{H}, \operatorname{Ar} H), 8.52(\mathrm{~d}, J=8.3 \mathrm{~Hz}, 1 \mathrm{H}, \operatorname{Ar} H), 8.08-8.01(\mathrm{~m}, 1 \mathrm{H}, \operatorname{Ar} H), 7.74-$ $7.65(\mathrm{~m}, 5 \mathrm{H}, \mathrm{Ar} H), 7.57$ (ddd, $J=8.3,7.0,1.3 \mathrm{~Hz}, 1 \mathrm{H}, \mathrm{Ar} H), 4.23\left(\mathrm{~s}, 3 \mathrm{H}, \mathrm{CO}_{2} \mathrm{CH}_{3}\right) ;{ }^{13} \mathbf{C} \mathbf{~ N M R}$ (101 MHz, Chloroform- $d$ ) $\delta 167.5,139.1,131.6,131.1,130.9,130.5,129.7,129.3,129.0,128.6$, $128.5,128.2,128.1,127.9,127.6,127.6,127.0,126.4,125.9,125.8,123.9,123.5,118.9,104.3$, 53.7; IR $\widetilde{\boldsymbol{v}}_{\max } 3064$ (w), 2952 (w), 2351 (w), 2221 (w), 1730 (s), 1610 (w), 1496 (m), $1442(\mathrm{~m})$, 1376 (m), 1245 (s), 1214 (s), 1154 (m), 1035 (m), 974 (m), 909 (w), 761 (s), 733 (s), 626 (m); HRMS (ESI) calcd for $\mathrm{C}_{25} \mathrm{H}_{16} \mathrm{NO}_{2}{ }^{+}[\mathrm{M}+\mathrm{H}]^{+} 362.1176$; found 362.1186.

\section{Methyl 5-cyanochrysene-6-carboxylate (37)}<smiles>CC(=O)c1c(C#N)c2c3ccccc3ccc2c2ccccc12</smiles>

Following the general procedure D, starting from methyl 2-(naphthalen-1-yl)-1-phenylcycloprop2-enecarboxylate (94) (90 $\mathrm{mg}, 0.30 \mathrm{mmol}, 1.0$ equiv.), the title compound 37 was obtained after purification by column chromatography on Biotage (Büchi flashpure cartridge $25 \mathrm{~g}$, Pentane:DCM 60:40) as a white solid (64 mg, $0.21 \mathrm{mmol}, 66 \%$ yield, $96 \%$ purity). ${ }^{29} \mathbf{R}_{\boldsymbol{f}} 0.38$ (Pentane:DCM 50:50); m.p = 135-136 ${ }^{\circ} \mathrm{C}$; ${ }^{1} \mathbf{H}$ NMR (400 MHz, Chloroform-d) $\delta 9.72(\mathrm{~d}, J=8.6 \mathrm{~Hz}, 1 \mathrm{H}, \operatorname{Ar} H$ ), $8.72(\mathrm{~d}, J=8.5 \mathrm{~Hz}, 1 \mathrm{H}, \operatorname{Ar} H), 8.59(\mathrm{~d}, J=9.1 \mathrm{~Hz}, 1 \mathrm{H}, \operatorname{Ar} H), 8.05(\mathrm{~d}, J=9.1 \mathrm{~Hz}, 1 \mathrm{H}, \operatorname{Ar} H), 8.00$ - $7.91(\mathrm{~m}, 2 \mathrm{H}, \mathrm{ArH}), 7.82$ (ddd, $J=8.4,6.8,1.4 \mathrm{~Hz}, 1 \mathrm{H}, \mathrm{ArH}), 7.78-7.62$ (m, 3H, ArH), 4.23 (s, $\left.3 \mathrm{H}, \mathrm{CO}_{2} \mathrm{CH}_{3}\right) ;{ }^{13} \mathbf{C}$ NMR (101 MHz, Chloroform-d) $\delta$ 167.9, 141.7, 133.0, 132.0, 130.7, 130.3, 130.0, 129.4, 128.9, 128.3, 127.8, 127.6, 126.8, 126.6, 125.1, 124.5, 123.9, 120.5, 119.6, 104.5, 53.9; IR $\widetilde{\boldsymbol{v}}_{\max } 3060(\mathrm{w}), 2953(\mathrm{w}), 2217(\mathrm{~m}), 1732(\mathrm{~s}), 1605(\mathrm{w}), 1568(\mathrm{w}), 1518(\mathrm{~m}), 1475(\mathrm{w})$, $1436(\mathrm{~m}), 1364(\mathrm{~m}), 1230(\mathrm{~s}), 1150(\mathrm{~m}), 1038(\mathrm{~m}), 965(\mathrm{~m}), 910(\mathrm{~m}), 859(\mathrm{~m}), 823(\mathrm{~m}), 754(\mathrm{~s})$, 735 (s), 654 (m); HRMS (ESI) calcd for $\mathrm{C}_{21} \mathrm{H}_{14} \mathrm{NO}_{2}{ }^{+}[\mathrm{M}+\mathrm{H}]^{+} 312.1019$; found 312.1018.

Methyl 14-cyanopicene-13-carboxylate (38) 


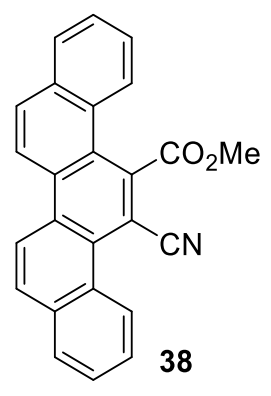

Following the general procedure D, starting from methyl 1,2-di(naphthalen-1-yl)cycloprop-2enecarboxylate (95) (105 $\mathrm{mg}, 0.300 \mathrm{mmol}, 1.0$ equiv.), the title compound $\mathbf{3 8}$ was obtained after purification by column chromatography on Biotage (Büchi flashpure cartridge $25 \mathrm{~g}$, Pentane:EtOAc 95:5) as a light brown solid (61 $\mathrm{mg}, 0.17 \mathrm{mmol}$, 54\% yield, 95\% purity). ${ }^{29} \mathbf{R}_{f} 0.42$ (Pentane:EtOAc 95:5); m.p = 158-159 ${ }^{\circ} \mathrm{C} ;{ }^{1} \mathbf{H}$ NMR (400 MHz, Chloroform- $d$ ) $\delta 9.64(\mathrm{dt}, J=$ 8.6, $0.9 \mathrm{~Hz}, 1 \mathrm{H}, \mathrm{Ar} H), 8.63(\mathrm{dd}, J=9.2,4.5 \mathrm{~Hz}, 2 \mathrm{H}, \operatorname{Ar} H), 8.47-8.38(\mathrm{~m}, 1 \mathrm{H}, \operatorname{Ar} H), 8.12-8.03$ $(\mathrm{m}, 2 \mathrm{H}, \operatorname{Ar} H), 8.01-7.95(\mathrm{~m}, 2 \mathrm{H}, \operatorname{Ar} H), 7.79$ (ddd, $J=8.6,7.0,1.6 \mathrm{~Hz}, 1 \mathrm{H}, \operatorname{Ar} H), 7.75-7.66(\mathrm{~m}$, $3 \mathrm{H}, \mathrm{ArH}), 4.20\left(\mathrm{~s}, 3 \mathrm{H}, \mathrm{CO}_{2} \mathrm{CH}_{3}\right) ;{ }^{13} \mathbf{C}$ NMR (101 MHz, Chloroform-d) $\delta$ 170.4, 138.9, 133.0, 132.8, 132.3, 131.9, 130.8, 130.1, 129.1, 129.1, 128.9, 128.8, 128.3, 127.9, 127.7, 127.6, 126.5, 125.9, 125.4, 124.8, 121.0, 120.7, 119.8, 105.6, 54.1; IR $\widetilde{\boldsymbol{v}}_{\max } 3052(\mathrm{w}), 2950(\mathrm{~m}), 2860(\mathrm{w}), 2218$ (m), $2106(\mathrm{~m}), 1731(\mathrm{~s}), 1607(\mathrm{~m}), 1515(\mathrm{~m}), 1439(\mathrm{~m}), 1371(\mathrm{~m}), 1337(\mathrm{~m}), 1265(\mathrm{~s}), 1227(\mathrm{~s})$, 1193 (m), 1164 (m), 1037 (m), 967 (m), 912 (s), 806 (s), 738 (s), 660 (m); HRMS (ESI) calcd for $\mathrm{C}_{25} \mathrm{H}_{16} \mathrm{NO}_{2}{ }^{+}[\mathrm{M}+\mathrm{H}]^{+} 362.1176$; found 362.1178 . 


\section{Crystal Structure of 9}

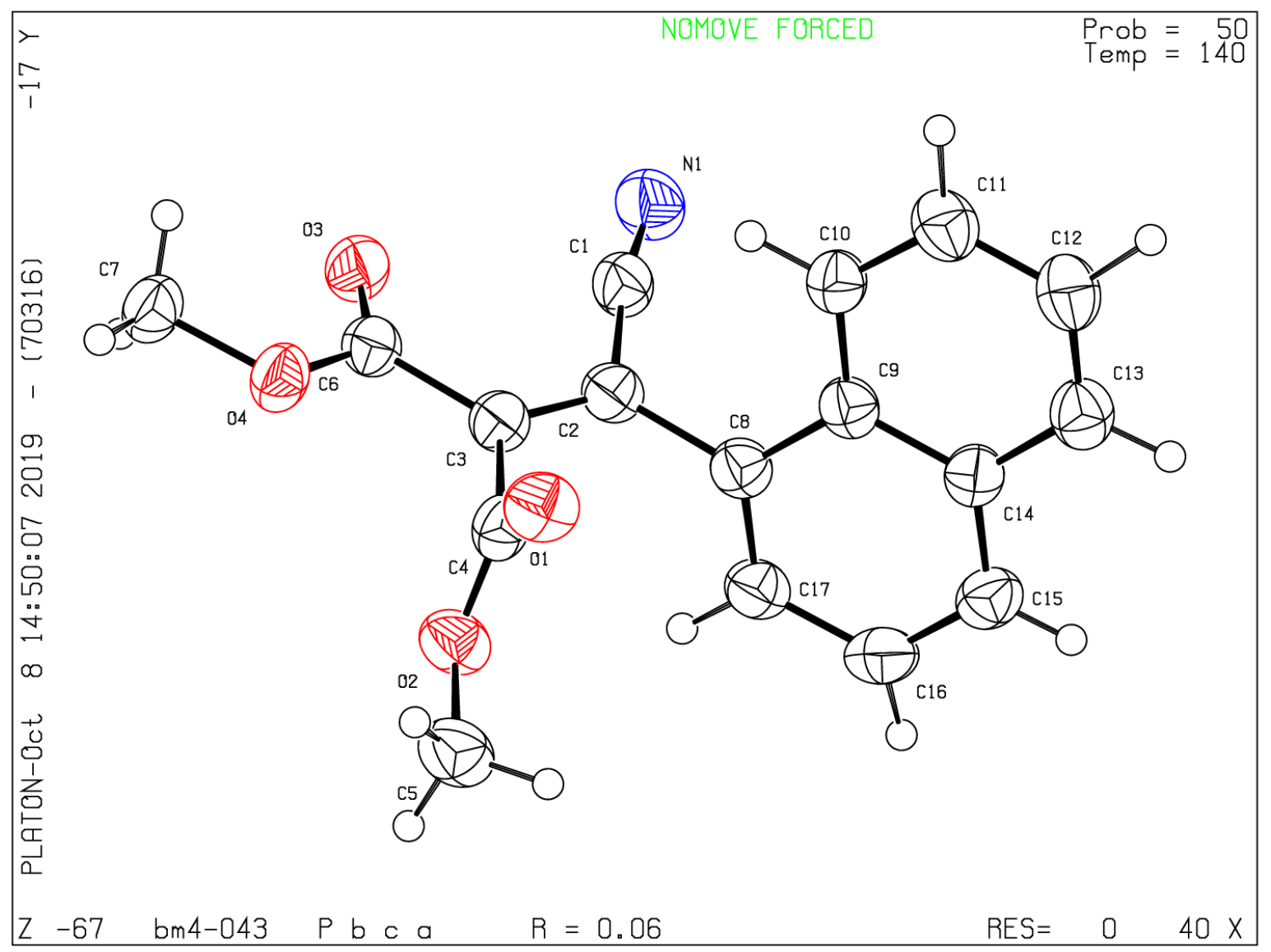

A single crystal was obtained by recrystallisation of 9 from a 1:1 DCM/Pentane mixture at room temperature. Supplementary crystallographic data for this compound have been deposited at Cambridge Crystallographic Data Centre (2027422) and can be obtained free of charge via www.ccdc.cam.ac.uk/data_request/cif. 


\section{Control Experiment and Characterization of 40}

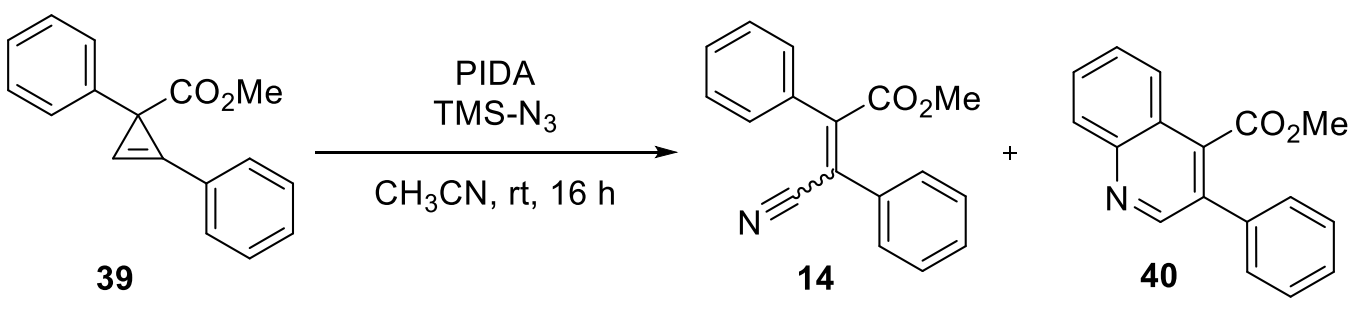

A $5 \mathrm{~mL}$ test tube was charged with cyclopropene 39 (50 mg, $0.20 \mathrm{mmol}, 1.0$ equiv.) and PIDA (129 mg, $0.400 \mathrm{mmol}, 2.0$ equiv.). The tube was sealed, evacuated and back-filled with nitrogen (3 times). Then $\mathrm{CH}_{3} \mathrm{CN}(2 \mathrm{~mL}, 0.1 \mathrm{M})$ and TMS-N $3(53 \mu \mathrm{L}, 0.40 \mathrm{mmol}, 2.0$ equiv.) were added via syringe and the resulting mixture was stirred at room temperature overnight. The reaction mixture was then filtered through a small pad of silica gel eluting with $\mathrm{Et}_{2} \mathrm{O}$, and the filtrate was concentrated under reduced pressure. The crude residue was purified by column chromatography $\left(\mathrm{SiO}_{2}\right.$, Pentane:EtOAc 90:10) affording compounds 14 (16 mg, $0.060 \mathrm{mmol}, 30 \%$ yield) as a pale yellow oil and compound 40 (18 mg, $0.068 \mathrm{mmol}, 34 \%$ yield) as a yellow oil. For 14 the characterization data is the same as the one reported in section 4. For compound 40: $\mathbf{R}_{\boldsymbol{f}} 0.42$ (Pentane:EtOAc 90:10); ${ }^{1} \mathbf{H}$ NMR (400 MHz, Chloroform-d) $\delta 9.02$ (s, 1H, HetArH), 8.19 (d, $J$ $=8.3 \mathrm{~Hz}, 1 \mathrm{H}, \operatorname{HetAr} H), 7.92(\mathrm{~d}, J=8.5 \mathrm{~Hz}, 1 \mathrm{H}, \operatorname{HetAr} H), 7.78$ (ddd, $J=8.4,6.9,1.4 \mathrm{~Hz}, 1 \mathrm{H}$, HetArH), 7.65 (ddd, $J=8.2,6.8,1.3 \mathrm{~Hz}, 1 \mathrm{H}$, HetArH), $7.53-7.45$ (m, 5H, ArH), $3.78(\mathrm{~s}, 3 \mathrm{H}$, $\left.\mathrm{CO}_{2} \mathrm{CH}_{3}\right) ;{ }^{13} \mathrm{C}$ NMR (101 MHz, Chloroform-d) $\delta 168.1,151.5,147.3,137.2,137.1,131.8,130.0$, 129.9, 129.0, 128.8, 128.5, 128.2, 125.1, 123.9, 52.7; IR $\widetilde{\boldsymbol{v}}_{\max } 3060$ (m), 2924 (m), 2855 (m), 1731 (s), $1611(\mathrm{~m}), 1575(\mathrm{~m}), 1500(\mathrm{~m}), 1442(\mathrm{~m}), 1375(\mathrm{~m}), 1321(\mathrm{~m}), 1255$ (s), 1223 (s), $1133(\mathrm{~m})$, $1096(\mathrm{~m}), 1029(\mathrm{~m}), 951$ (m), 765 (s), 705 (s) ; HRMS (ESI) calcd for $\mathrm{C}_{17} \mathrm{H}_{14} \mathrm{NO}_{2}{ }^{+}[\mathrm{M}+\mathrm{H}]^{+}$ 264.1019; found 264.1020. 


\section{Spectra of New Compounds}

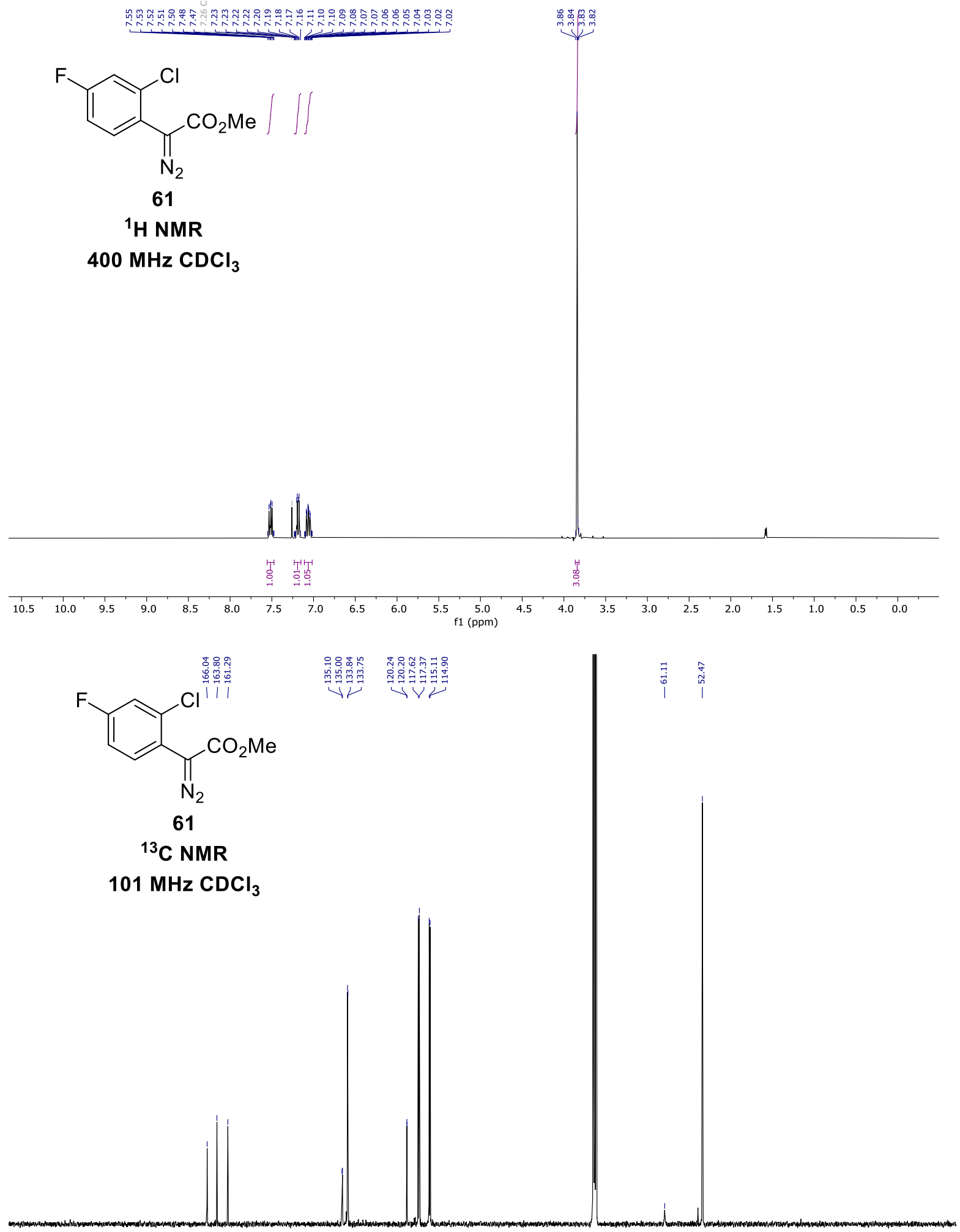

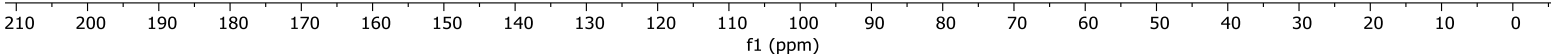


<smiles>COC(=O)C(=N)c1ccc(F)cc1Cl</smiles>

61

${ }^{19} \mathrm{~F}$ NMR

$376 \mathrm{MHz} \mathrm{CDCl}_{3}$

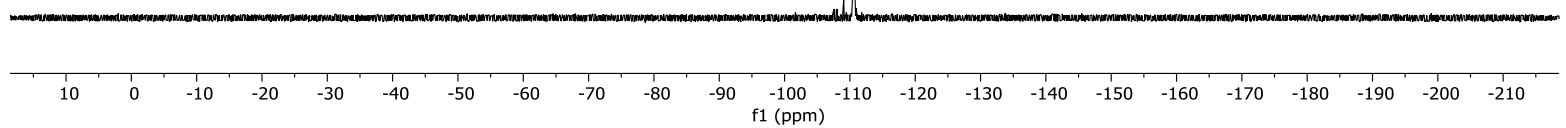




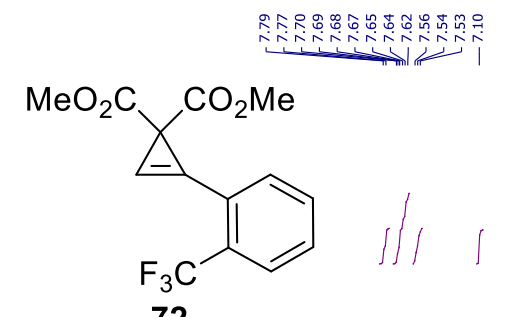

72

${ }^{1} \mathrm{H}$ NMR

$400 \mathrm{MHz} \mathrm{CDCl}_{3}$
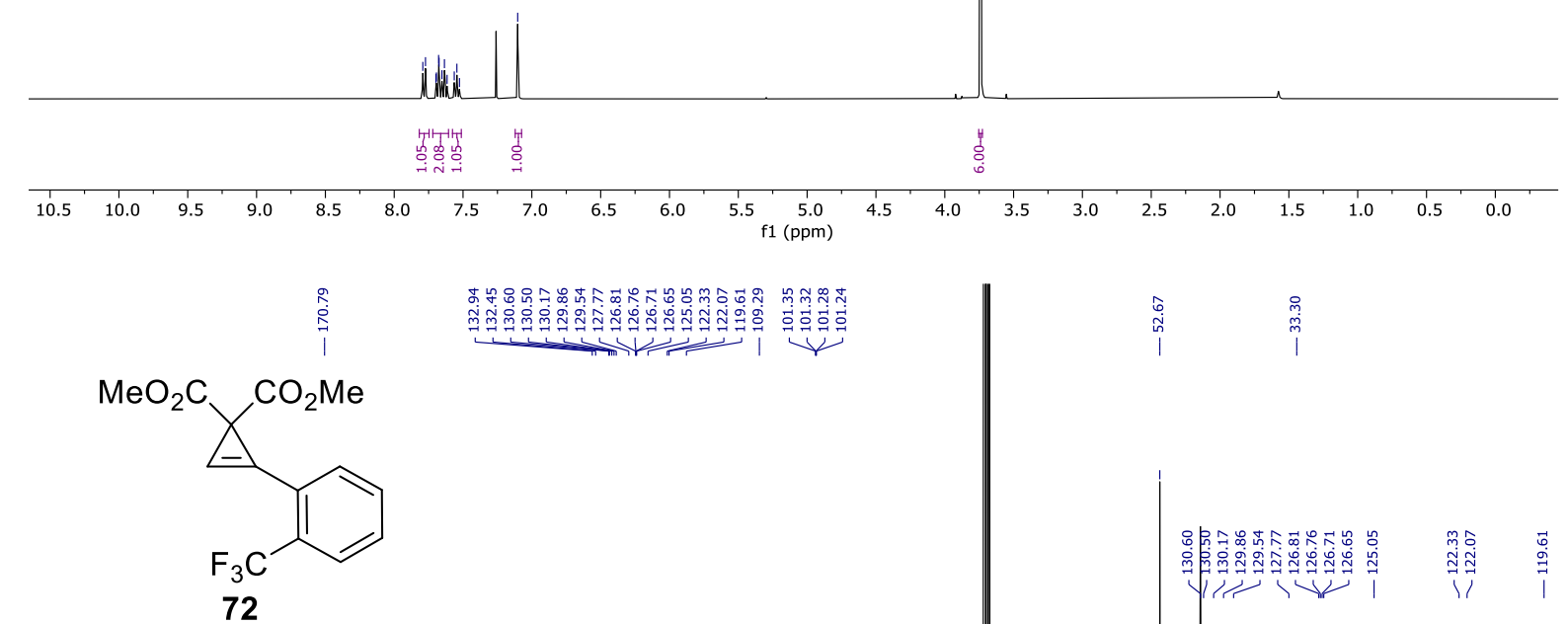

${ }^{13} \mathrm{C}$ NMR

$101 \mathrm{MHz} C D C l_{3}$

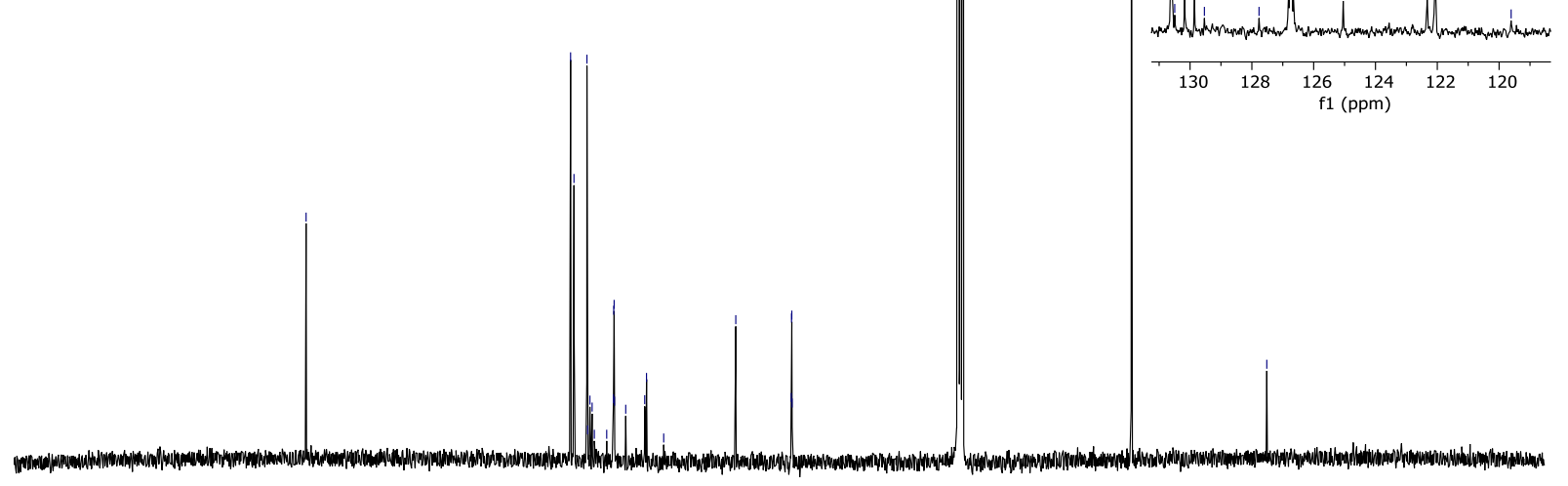

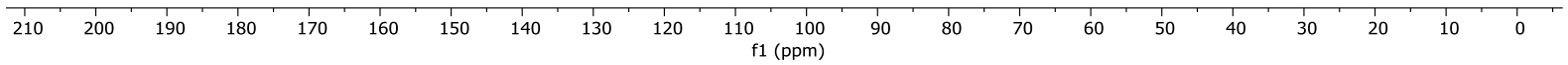




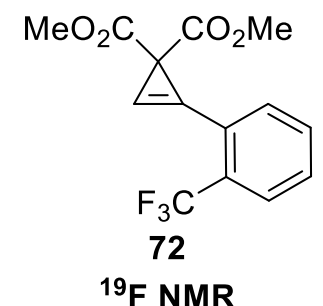

$400 \mathrm{MHz} \mathrm{CDCl}_{3}$

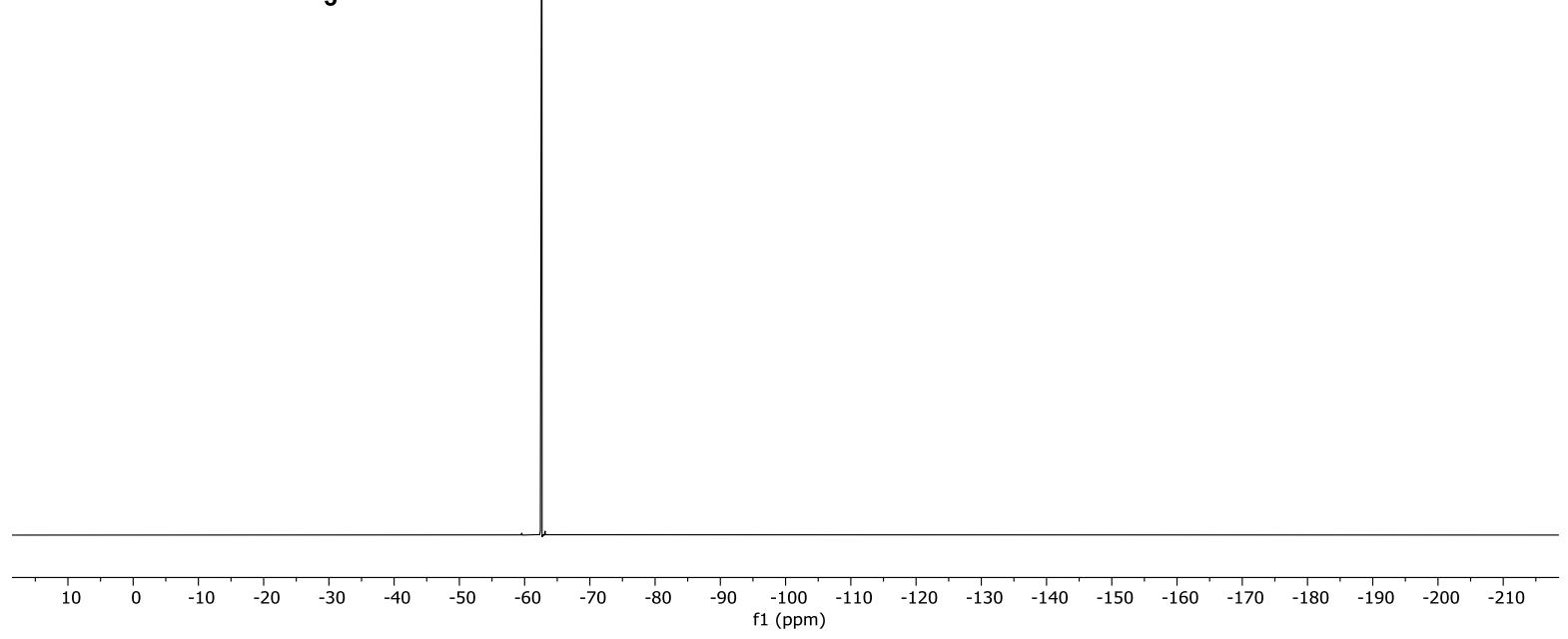




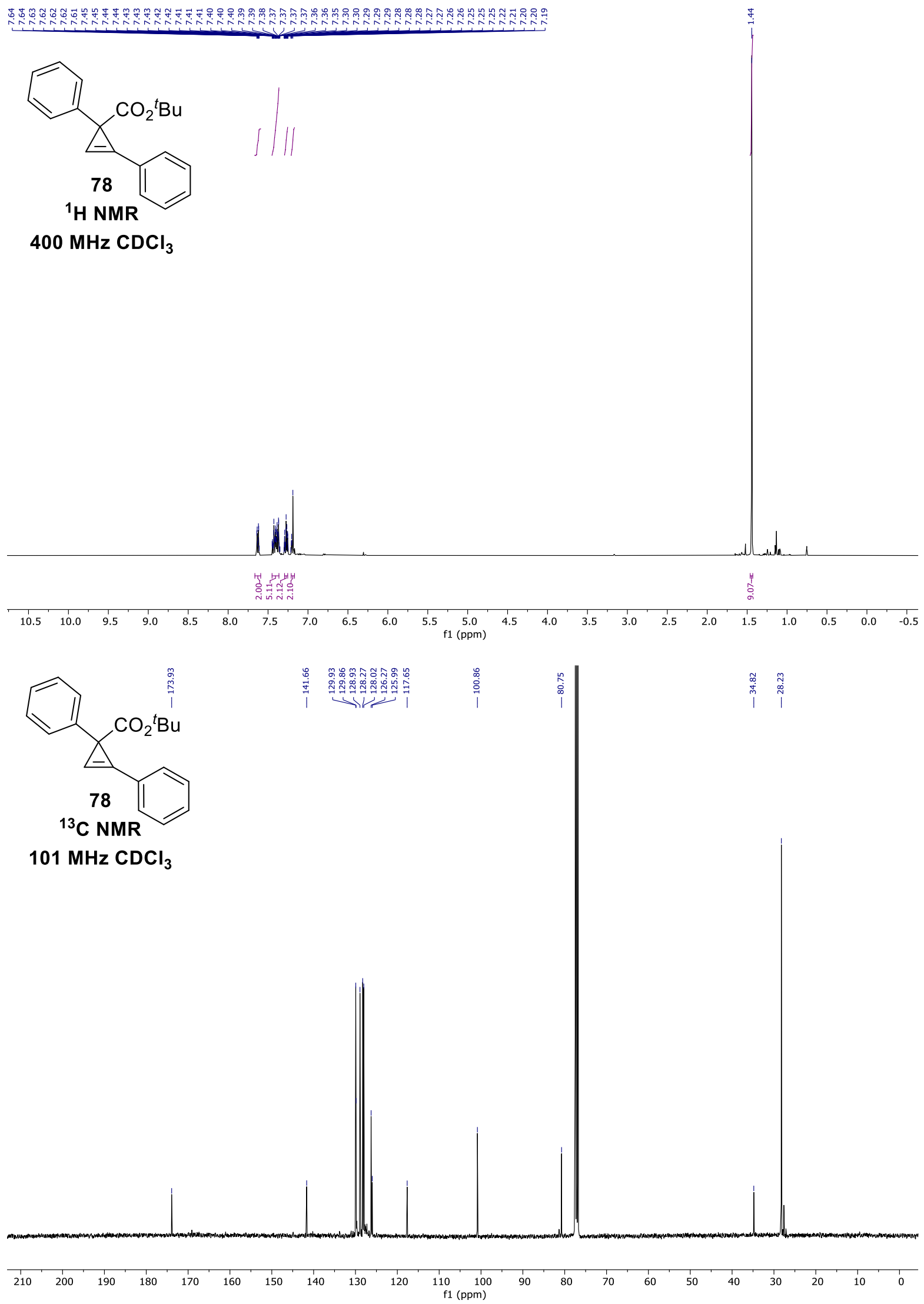




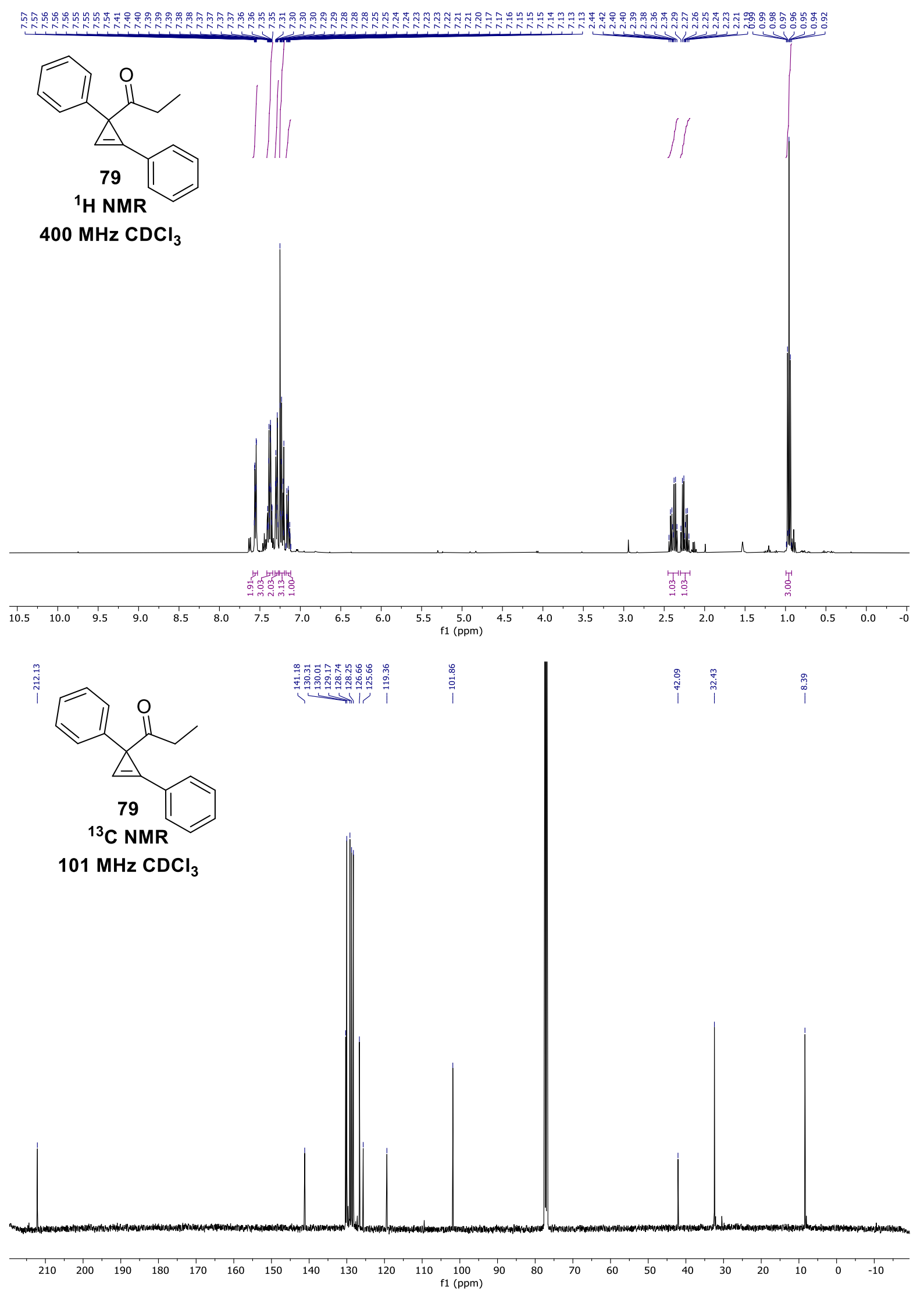



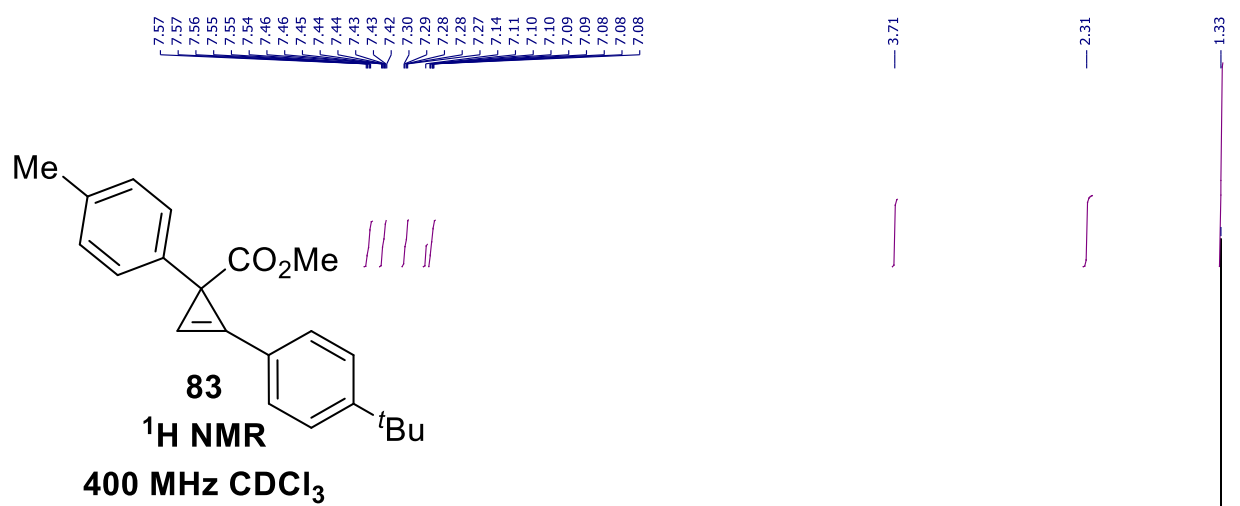

$\mathrm{MHz} \mathrm{CDCl}_{3}$
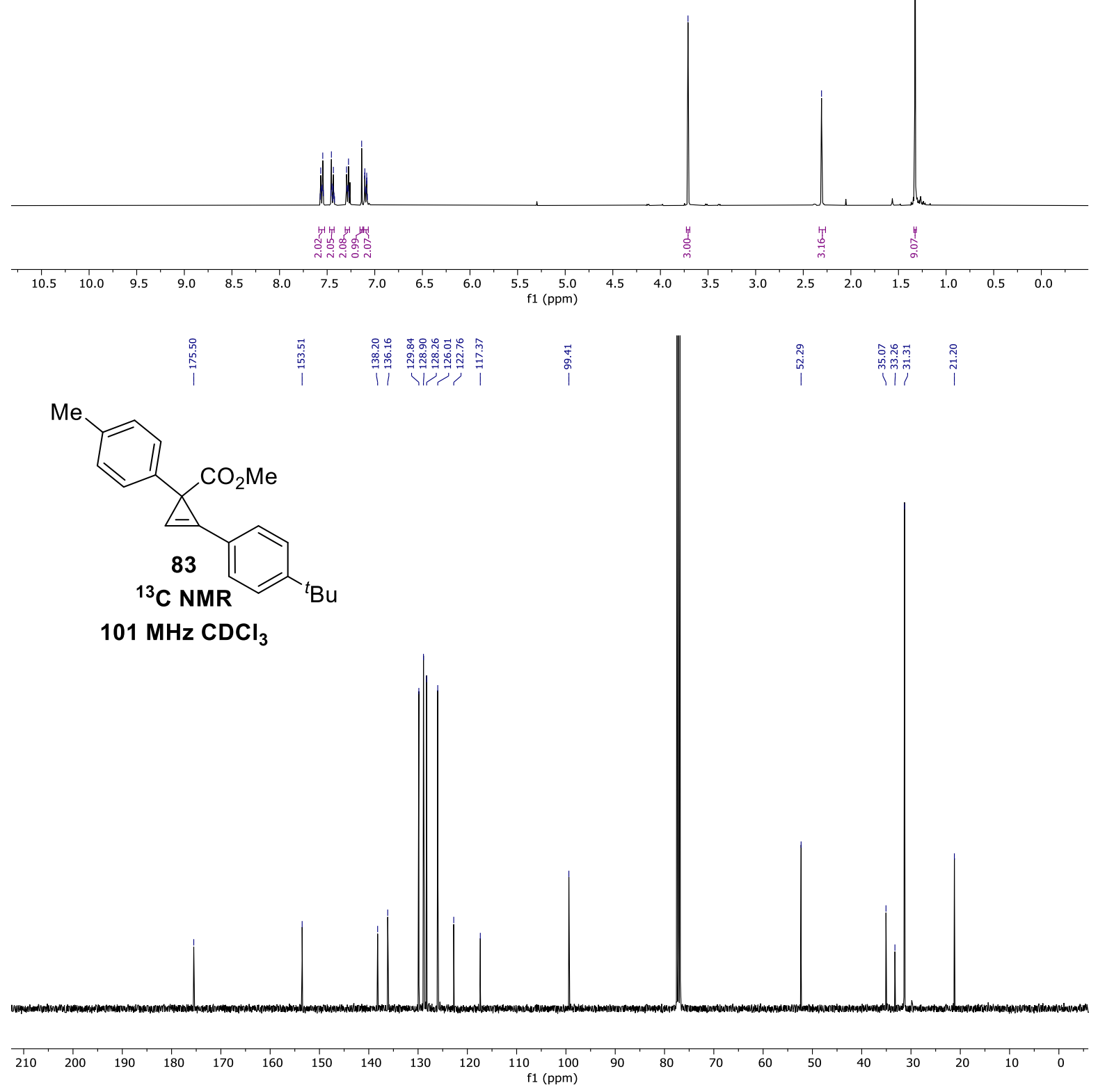


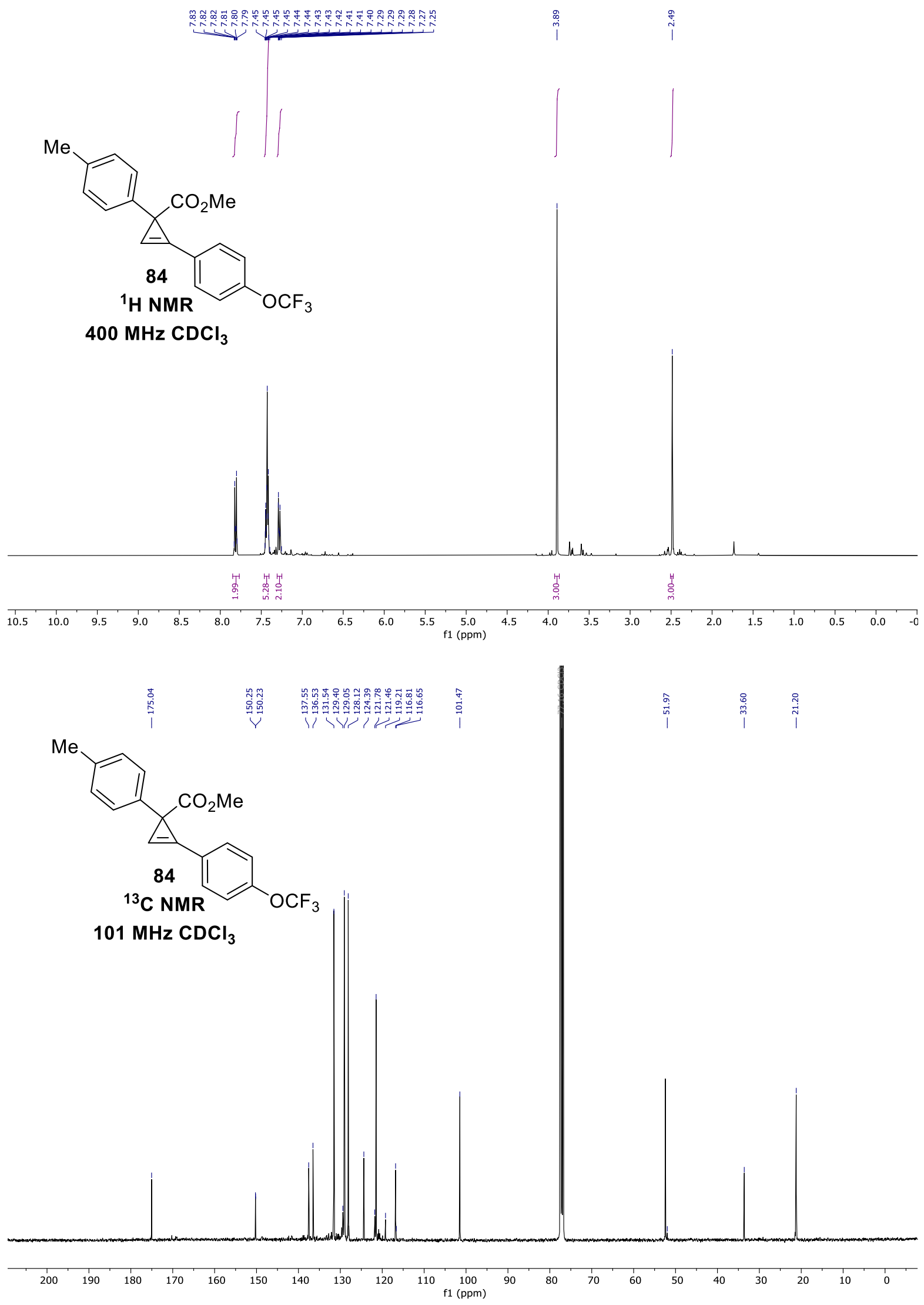




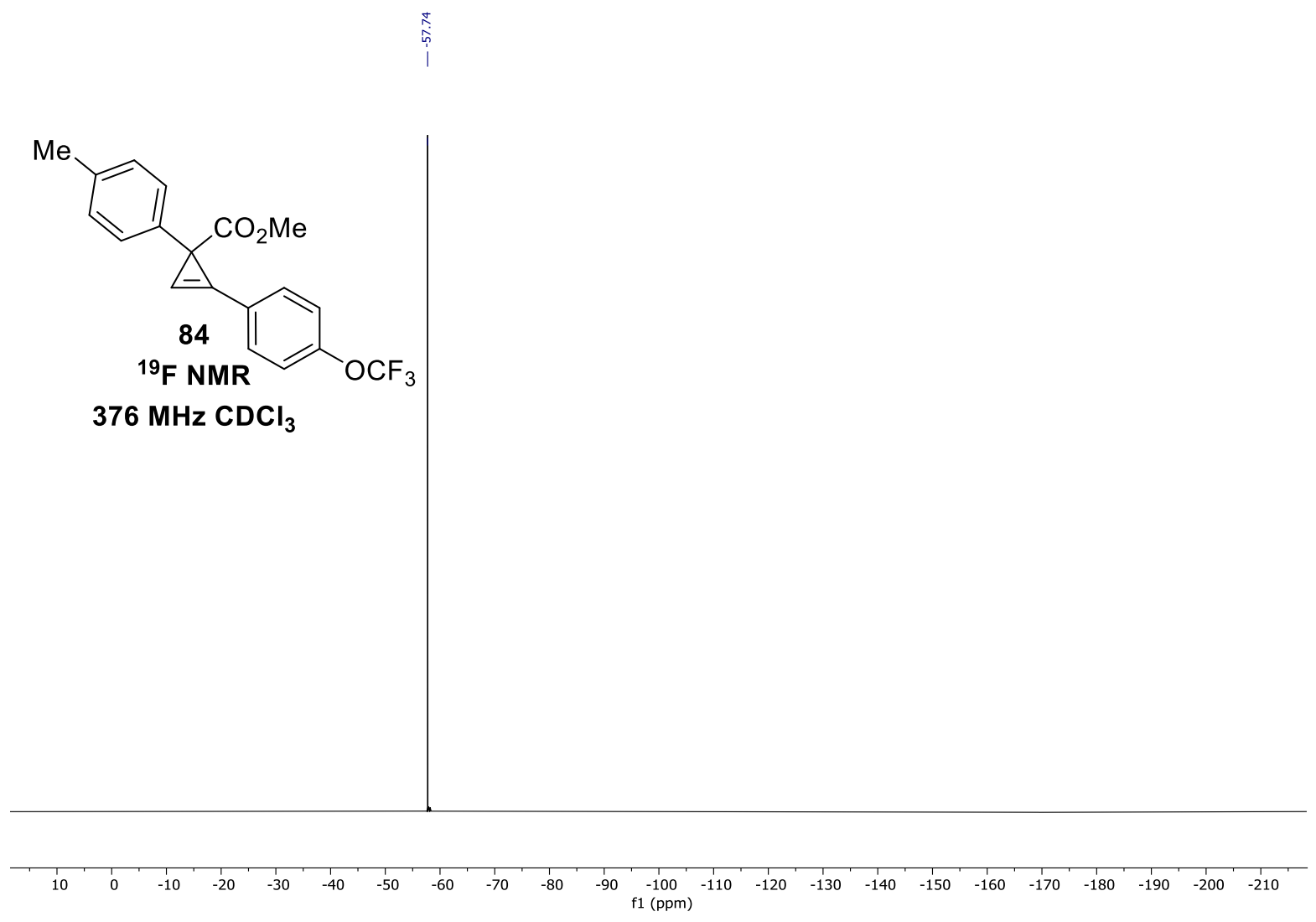




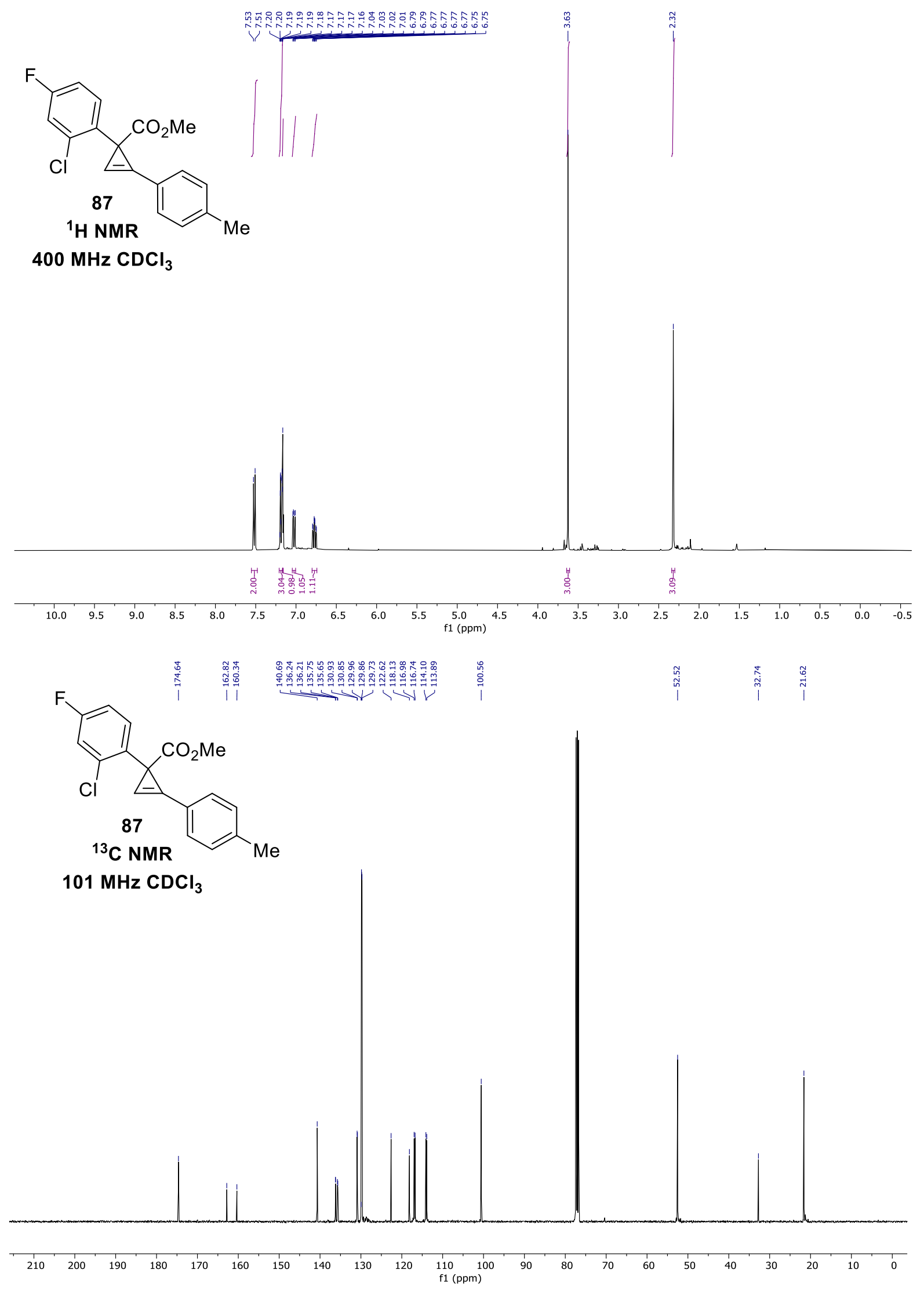



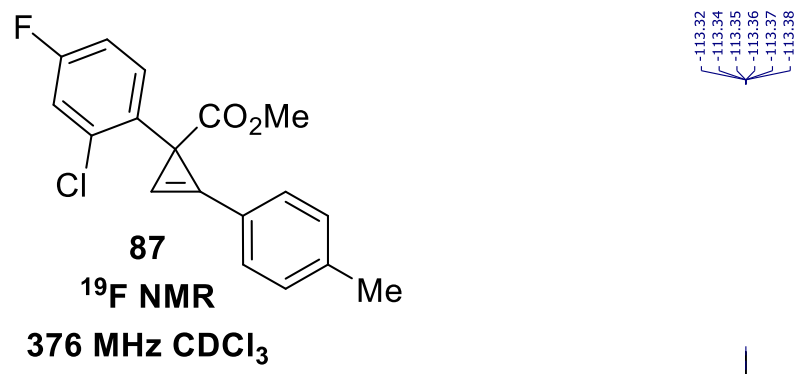

$376 \mathrm{MHz} \mathrm{CDCl}_{3}$

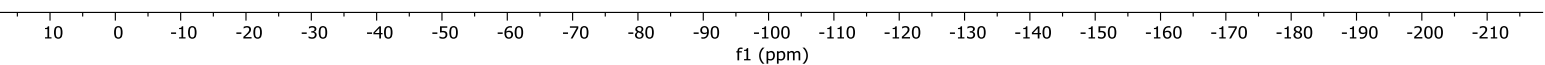




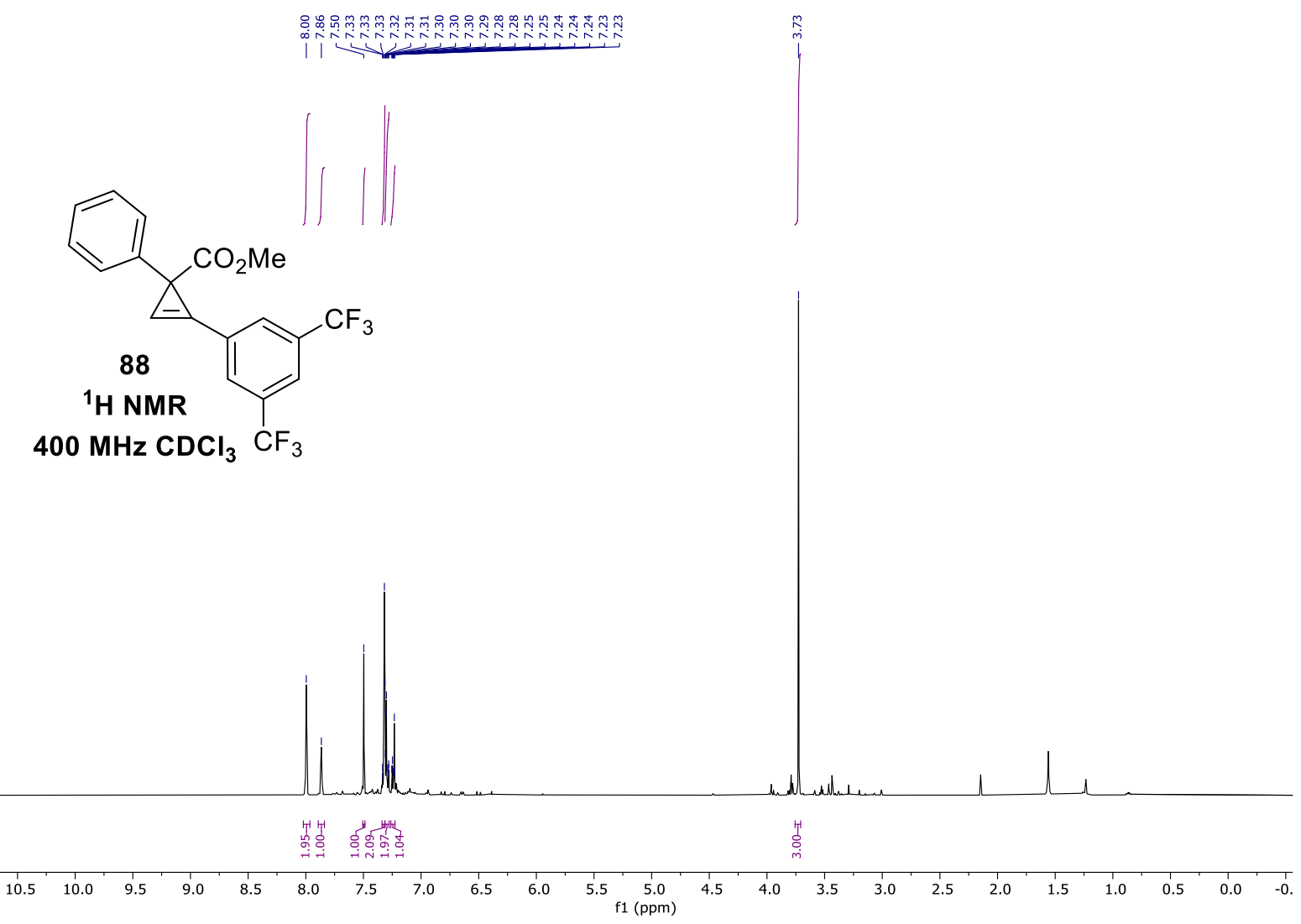

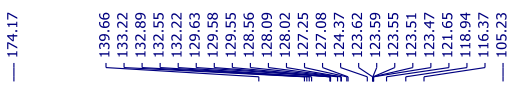

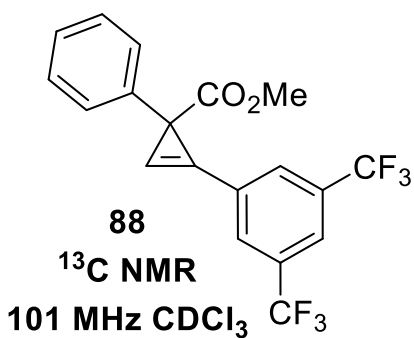

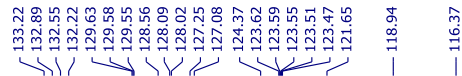

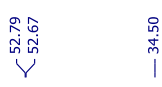




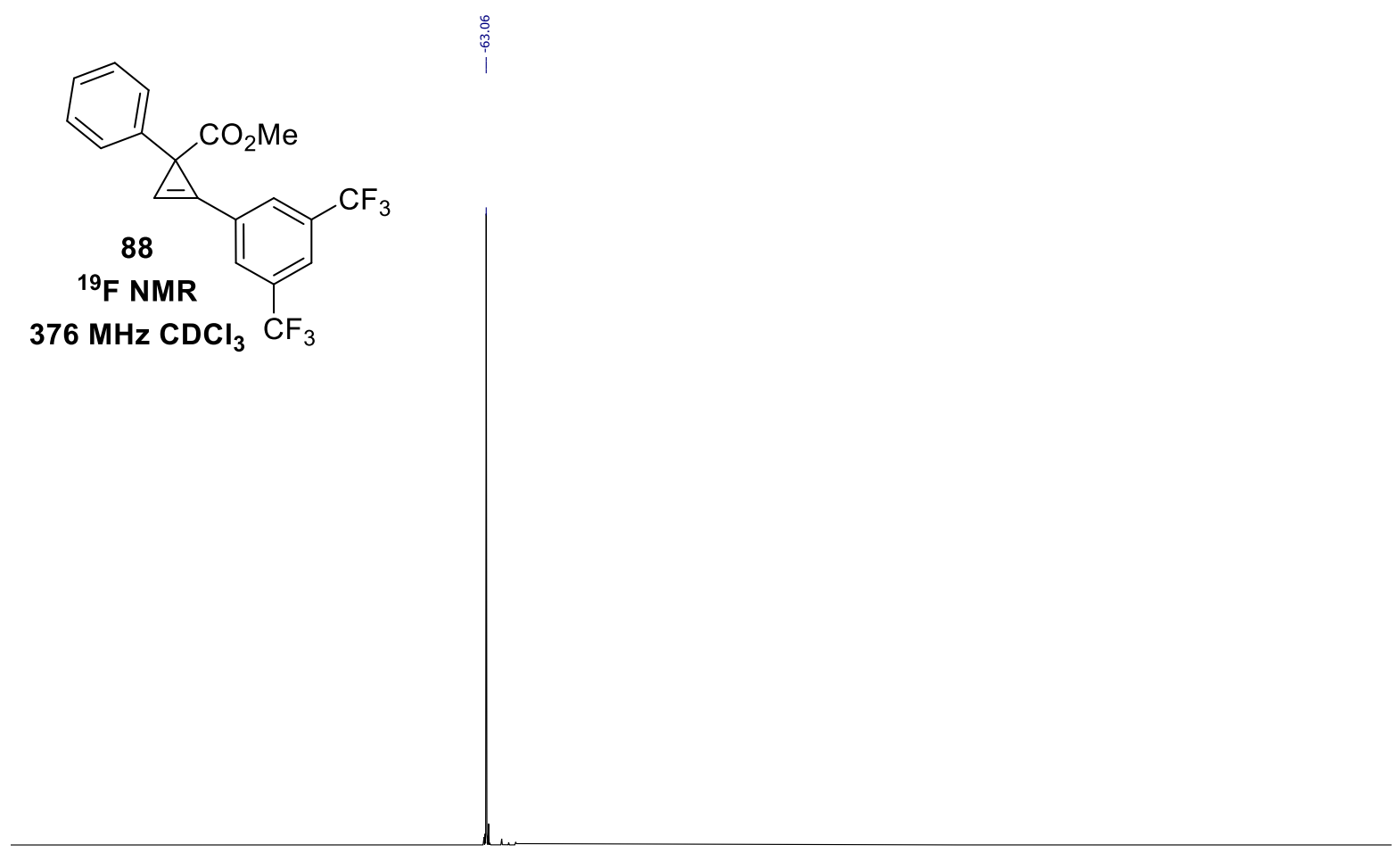

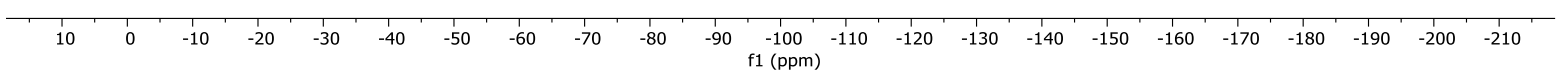



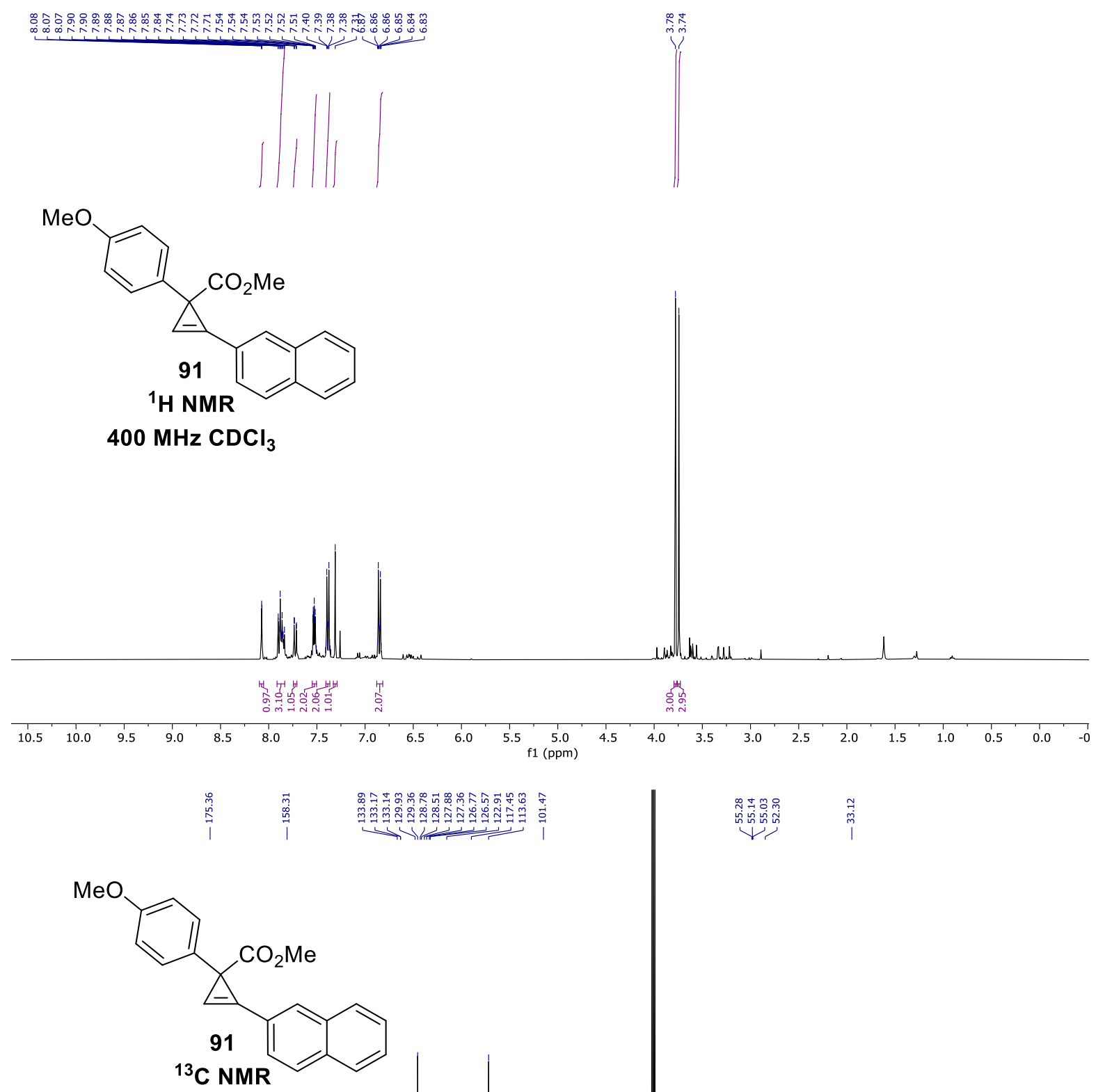

$101 \mathrm{MHz} \mathrm{CDCl}_{3}$

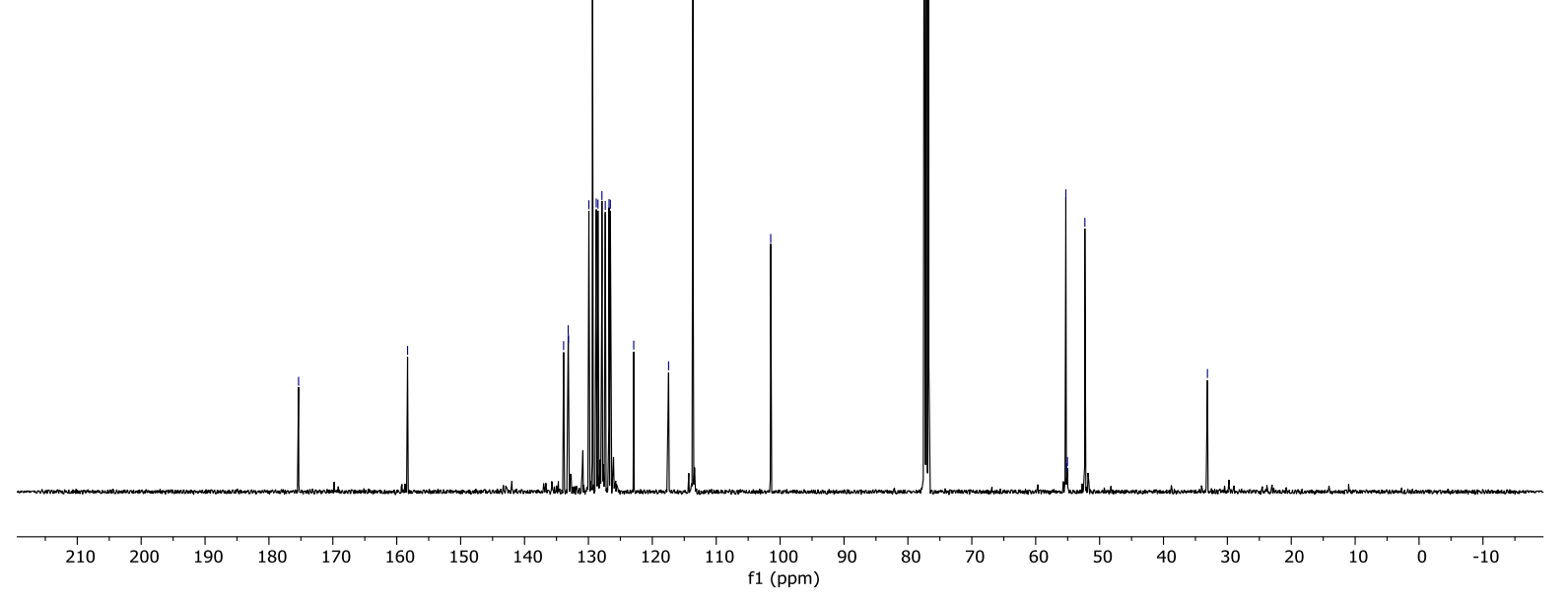



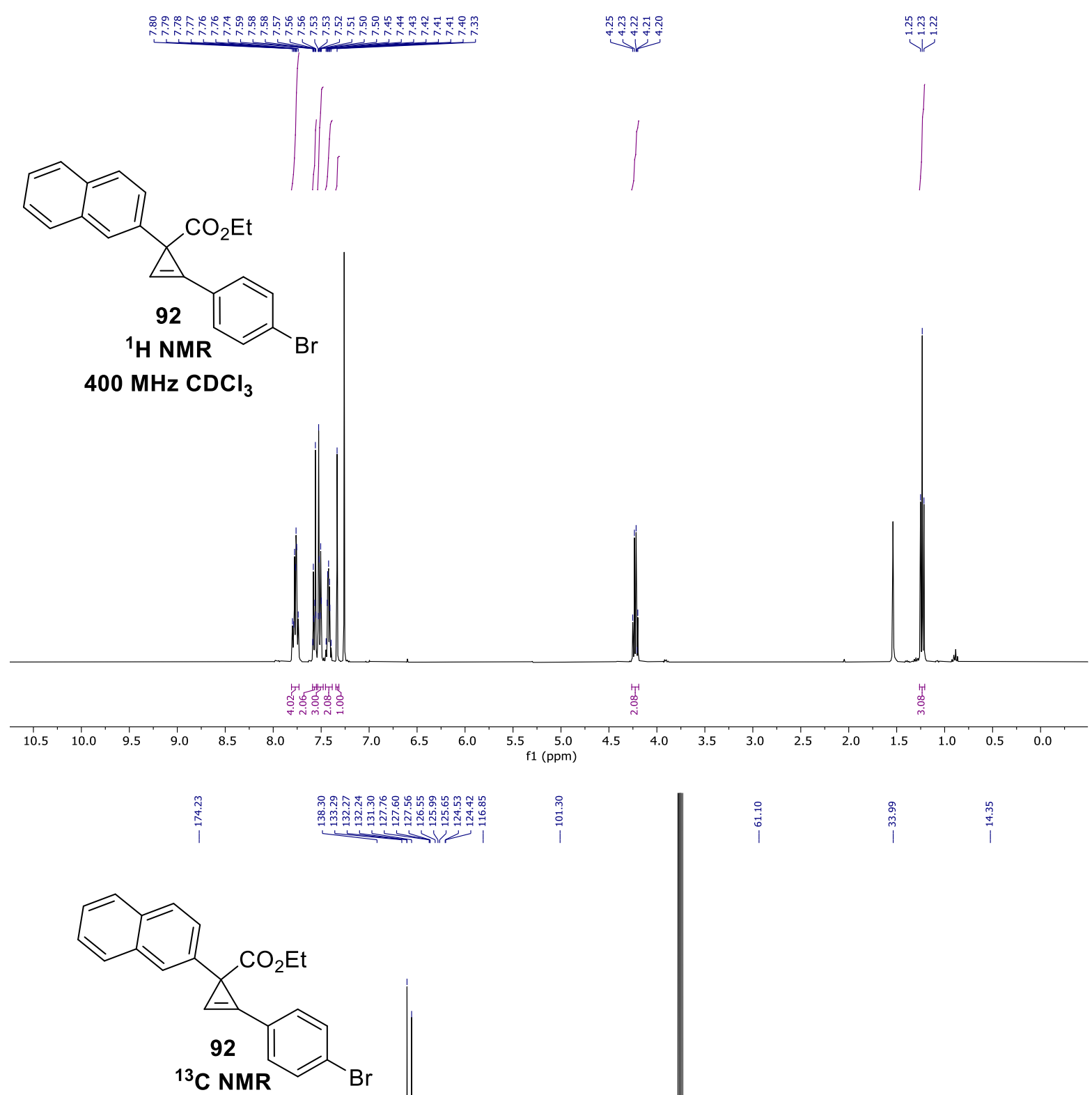

$101 \mathrm{MHz} \mathrm{CDCl}_{3}$
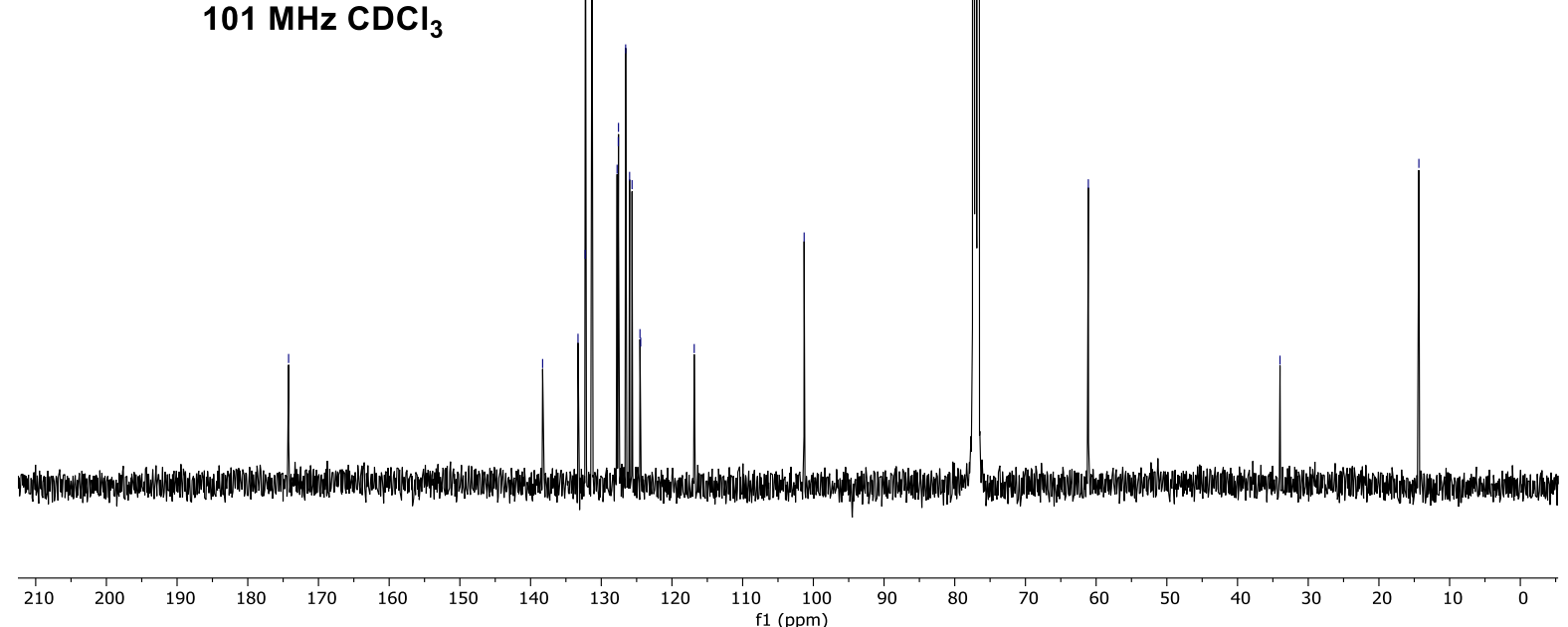

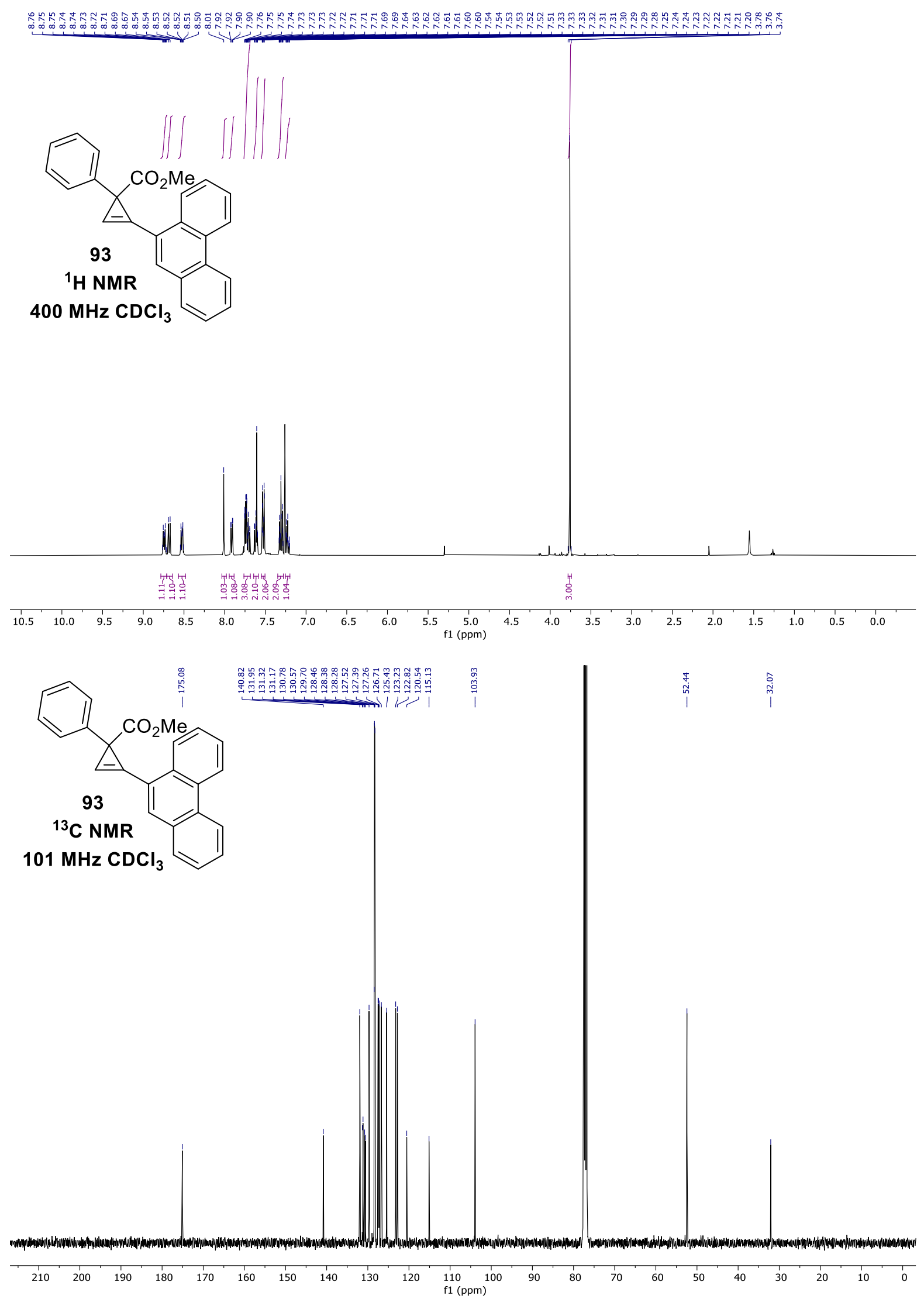


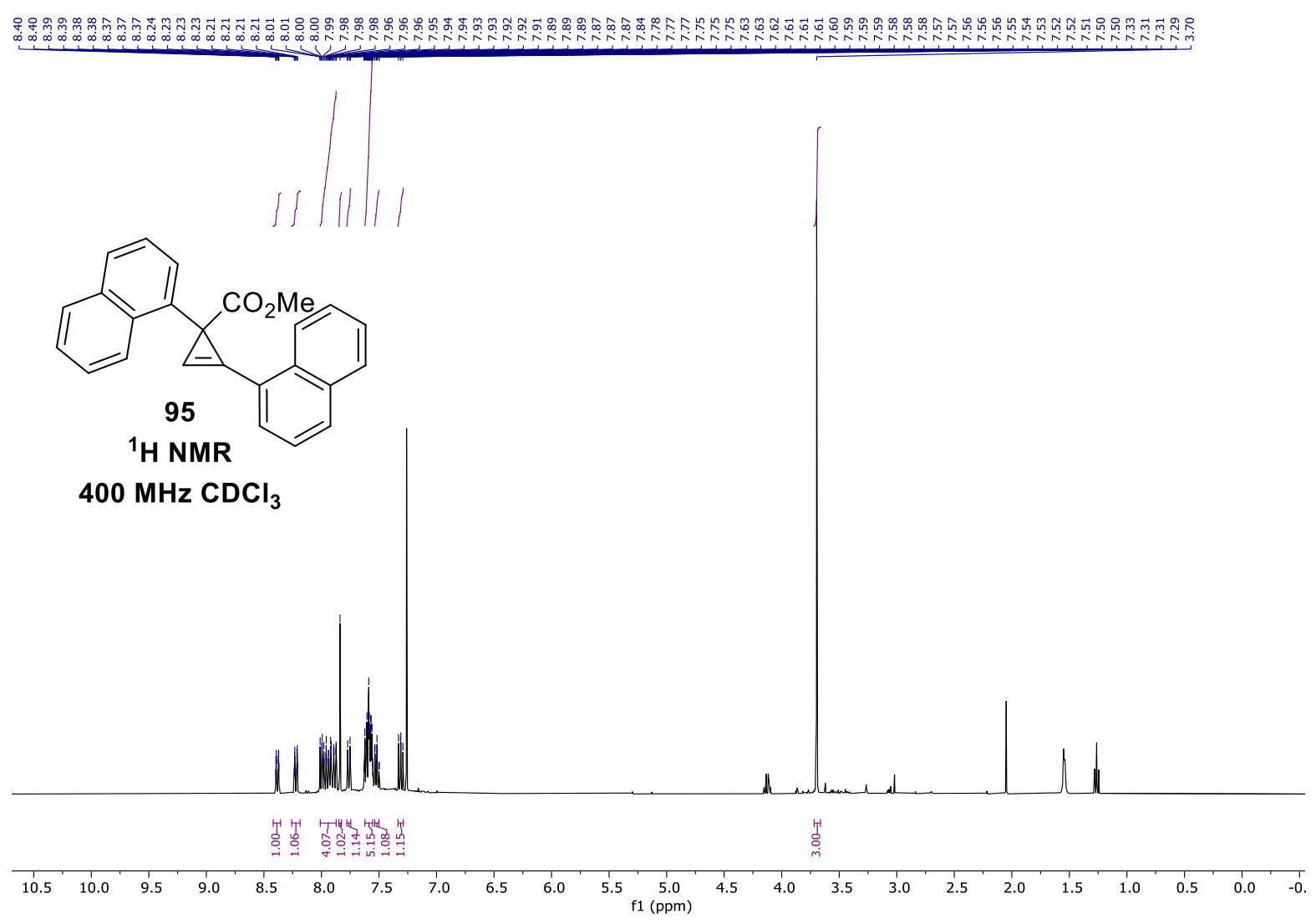

\begin{tabular}{ll||l}
\hline \\
\hline
\end{tabular}<smiles>COC(=O)C1(c2cccc3ccccc23)C=C1c1cccc2ccccc12</smiles>

${ }^{13} \mathrm{C}$ NMR

$101 \mathrm{MHz} \mathrm{CDCl}_{3}$

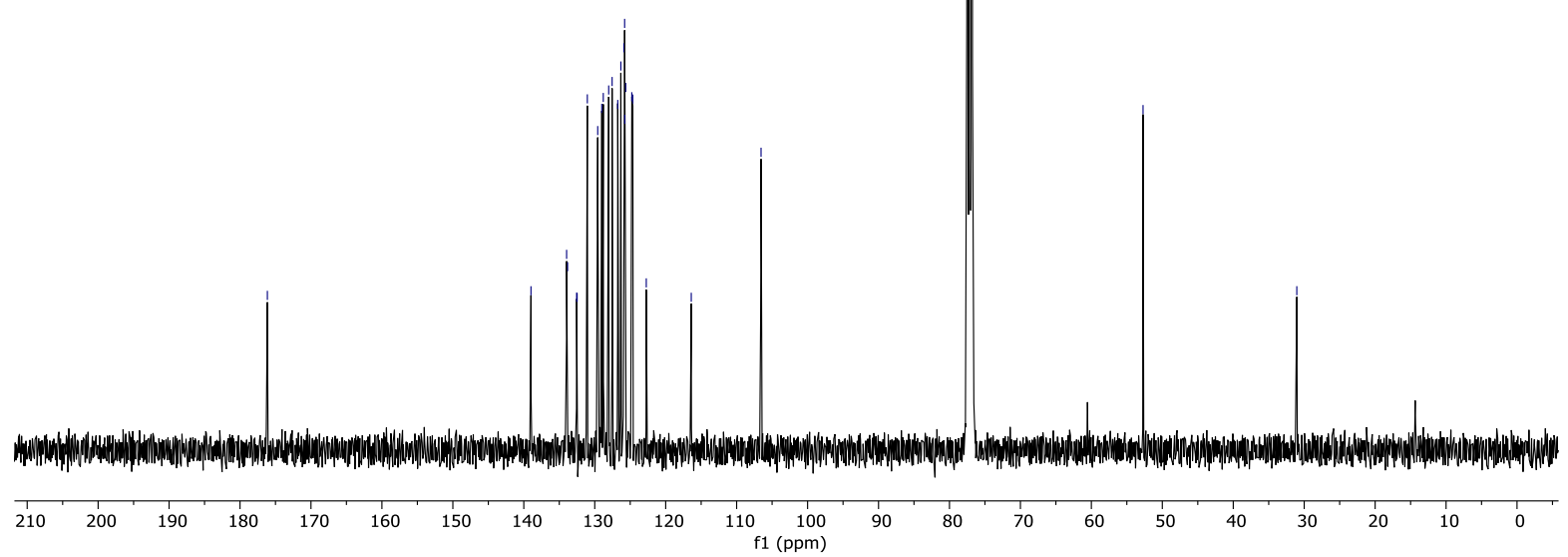



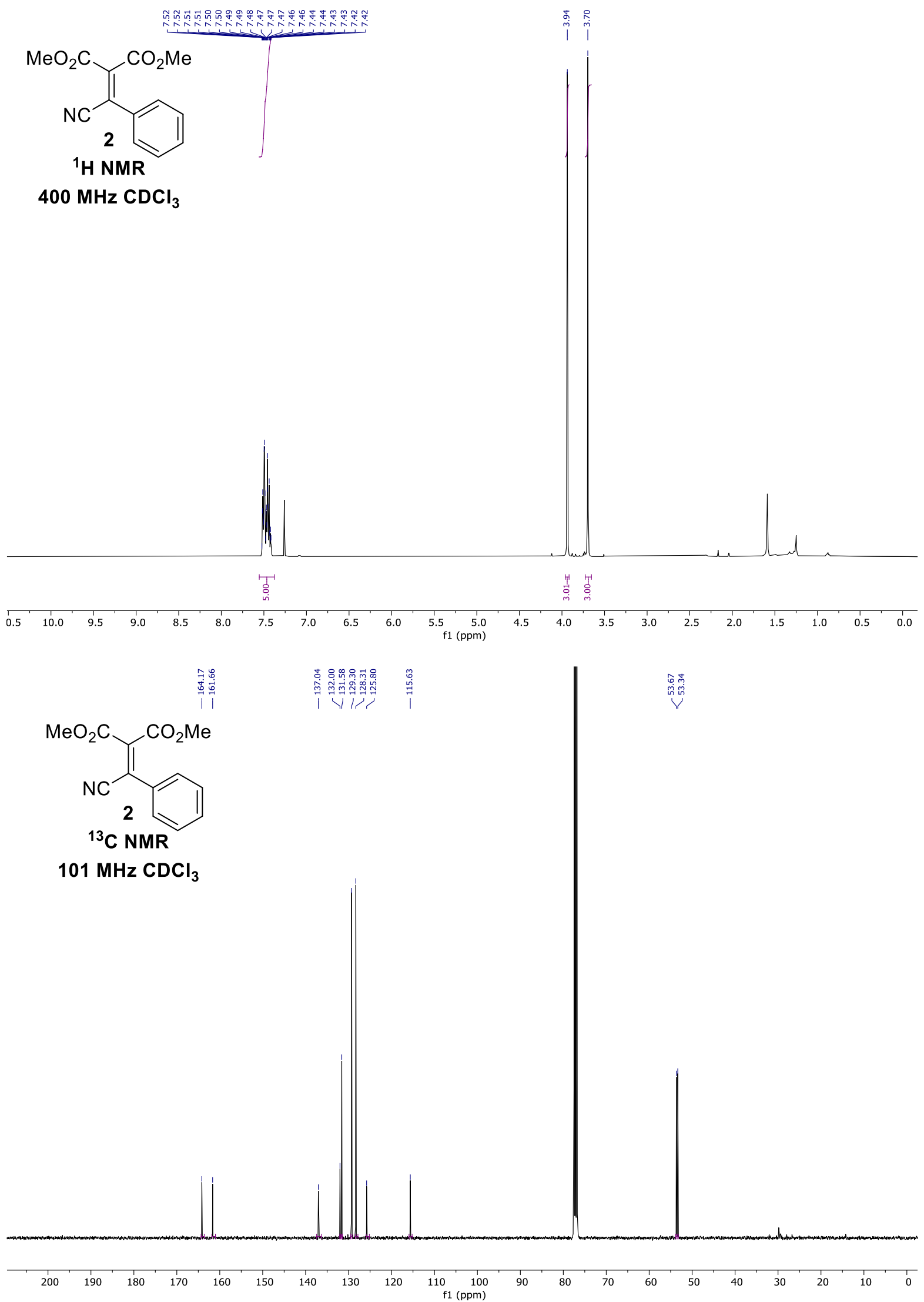


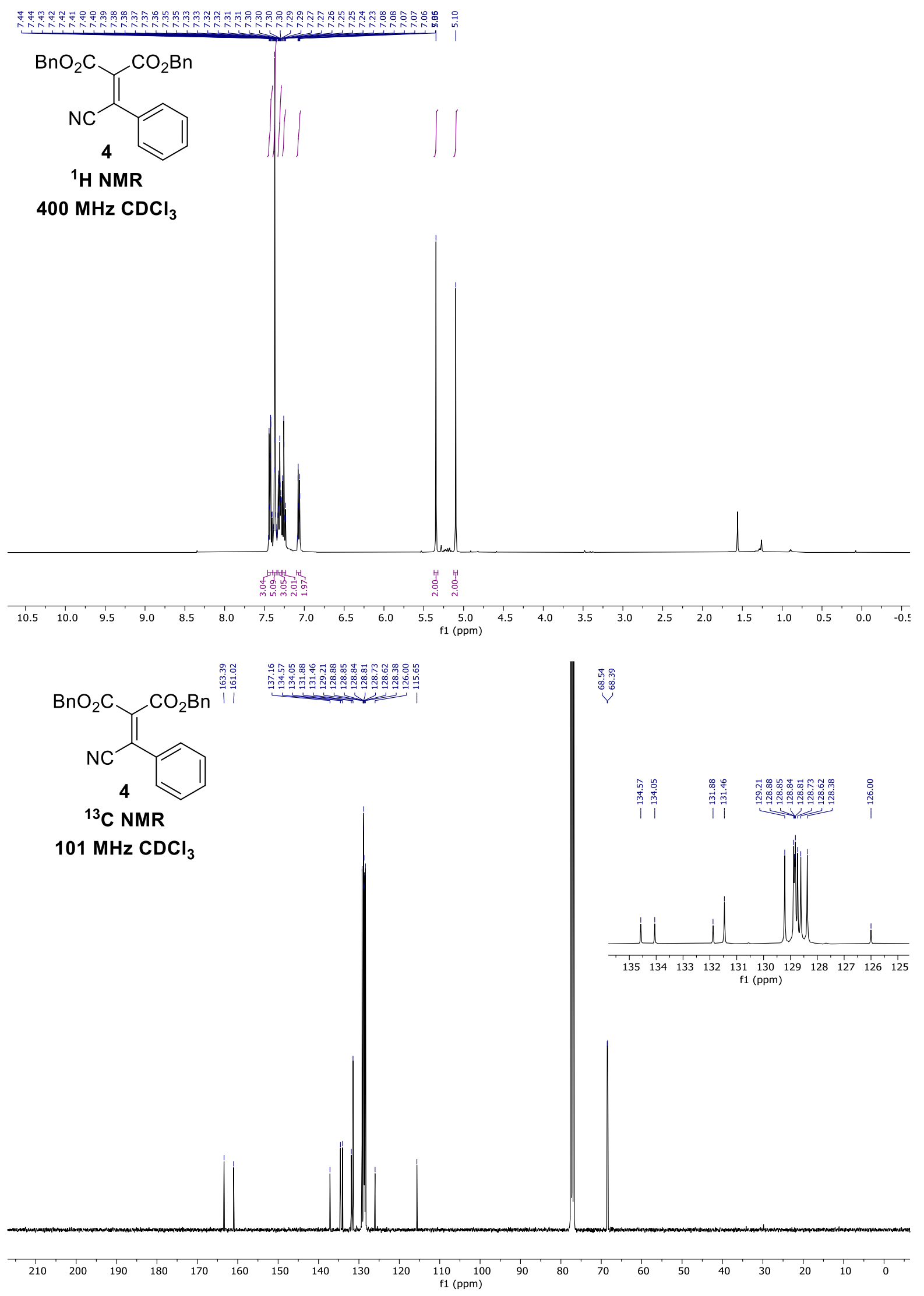



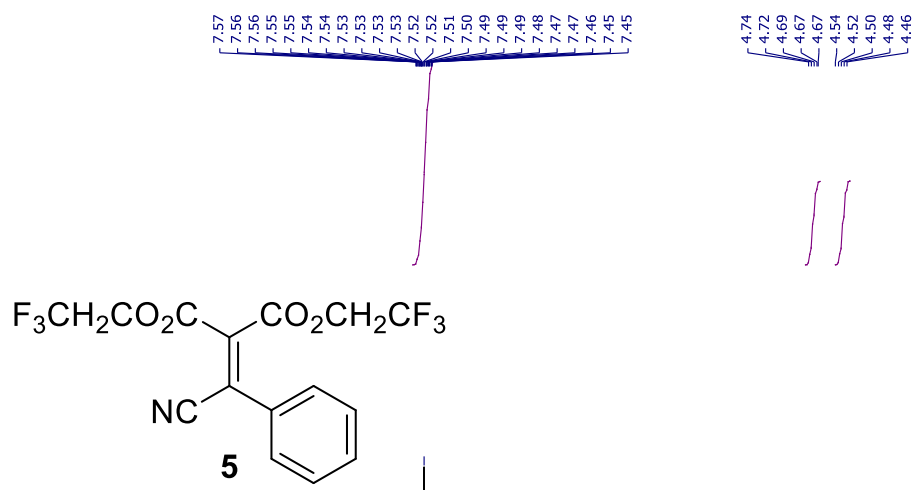

${ }^{1} \mathrm{H}$ NMR $400 \mathrm{MHz} \mathrm{CDCl}_{3}$
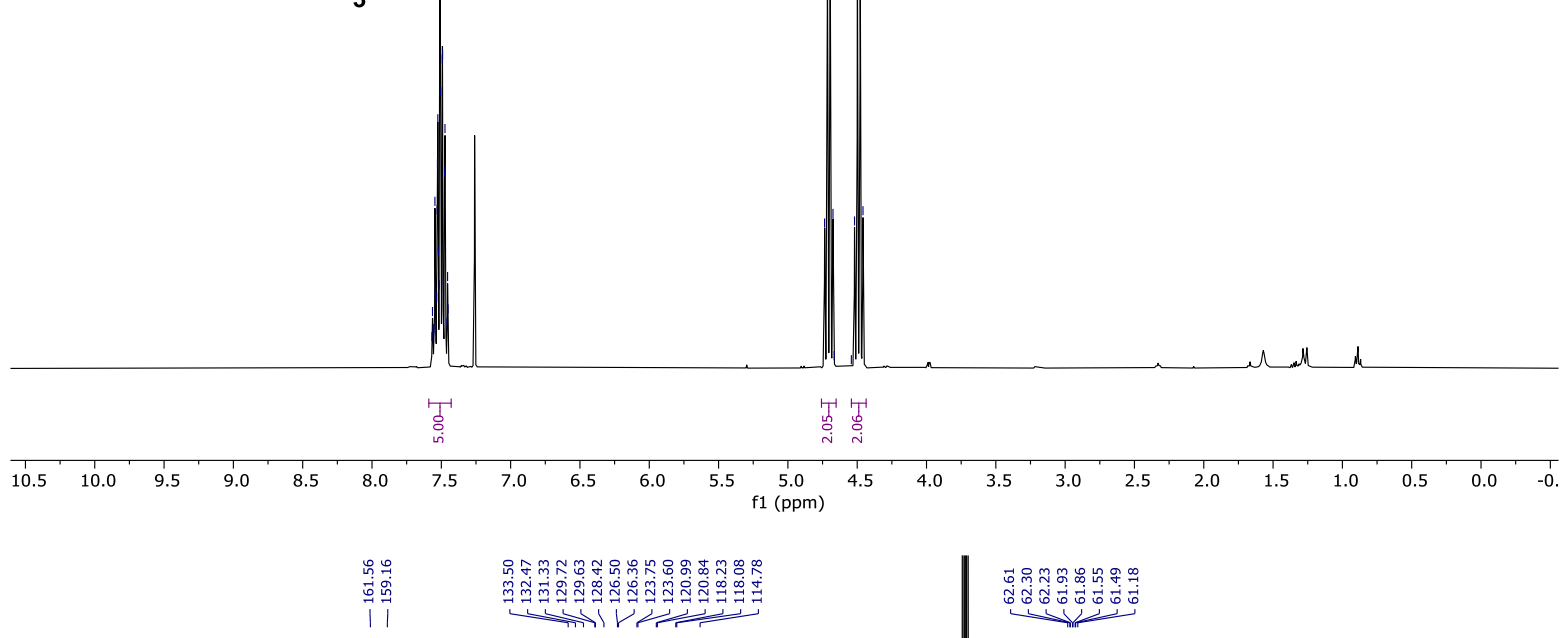

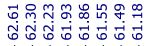

$\mathrm{F}_{3} \mathrm{CH}_{2} \mathrm{CO}_{2} \mathrm{C}-\mathrm{CO}_{2} \mathrm{CH}_{2} \mathrm{CF}_{3}$<smiles>C=C(C#N)c1ccccc1</smiles>

${ }^{13} \mathrm{C}$ NMR $101 \mathrm{MHz}^{\mathrm{CDCl}_{3}}$

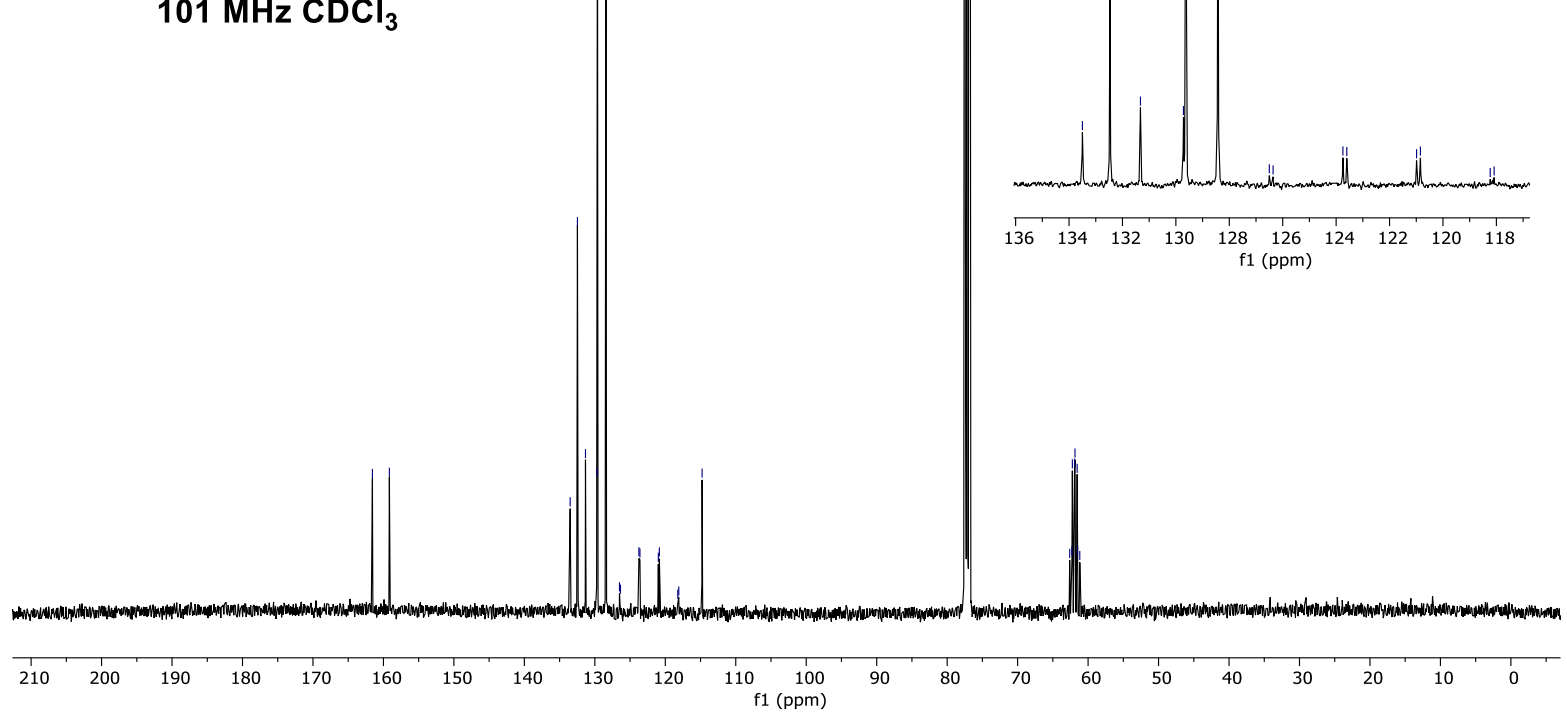




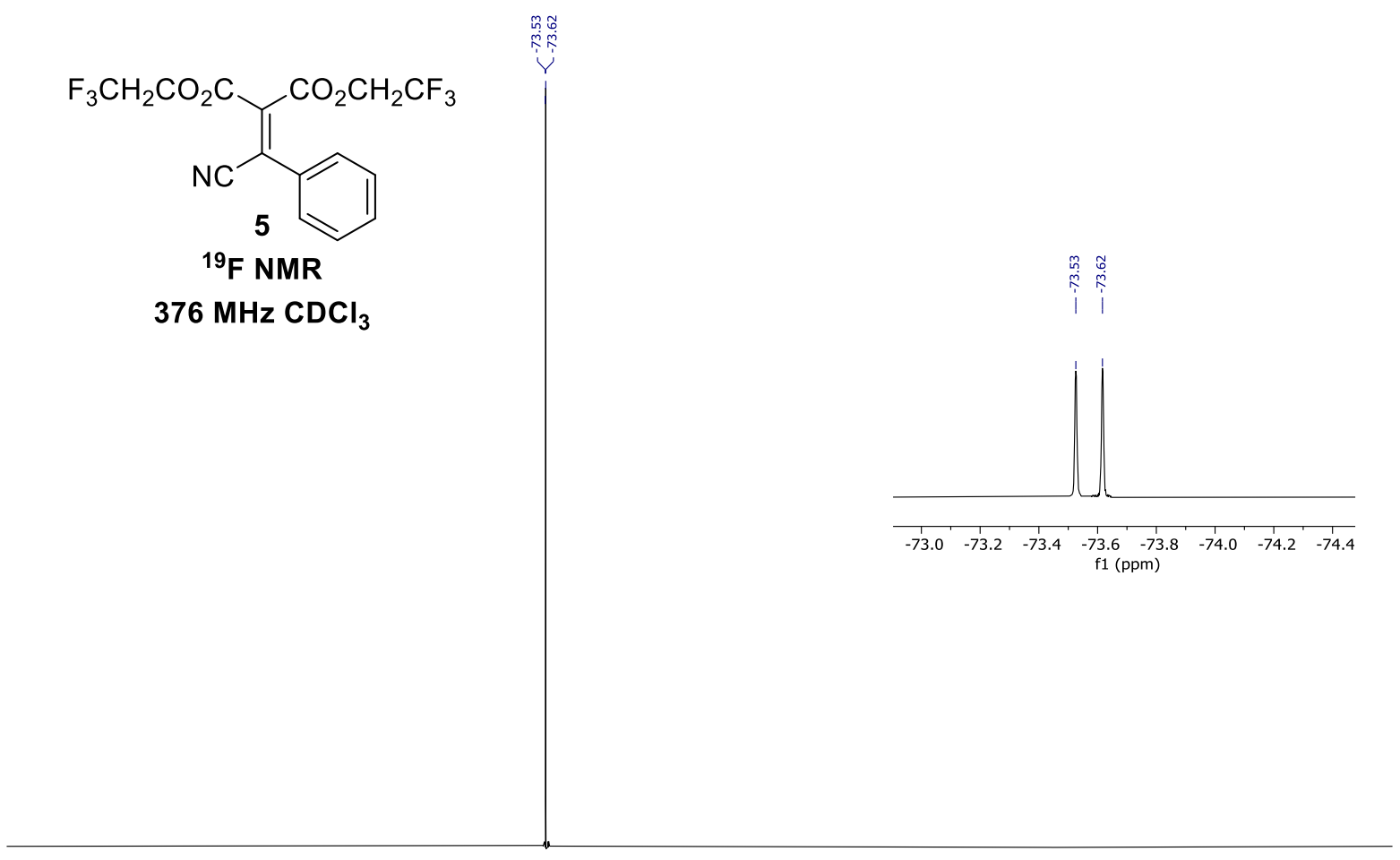

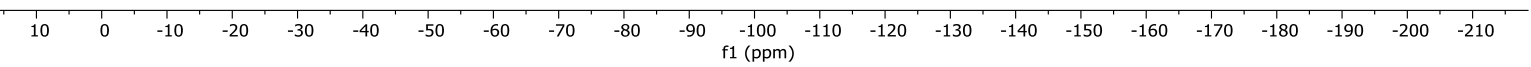



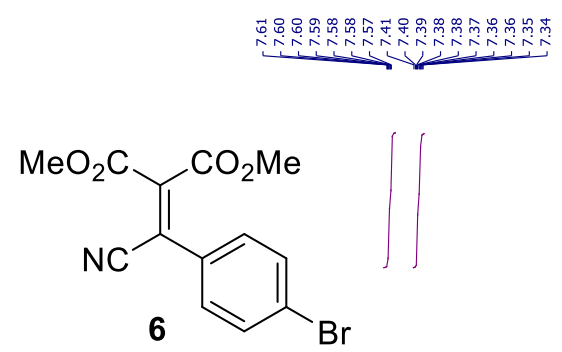

${ }^{1} \mathrm{H}$ NMR

$400 \mathrm{MHz} \mathrm{CDCl}_{3}$

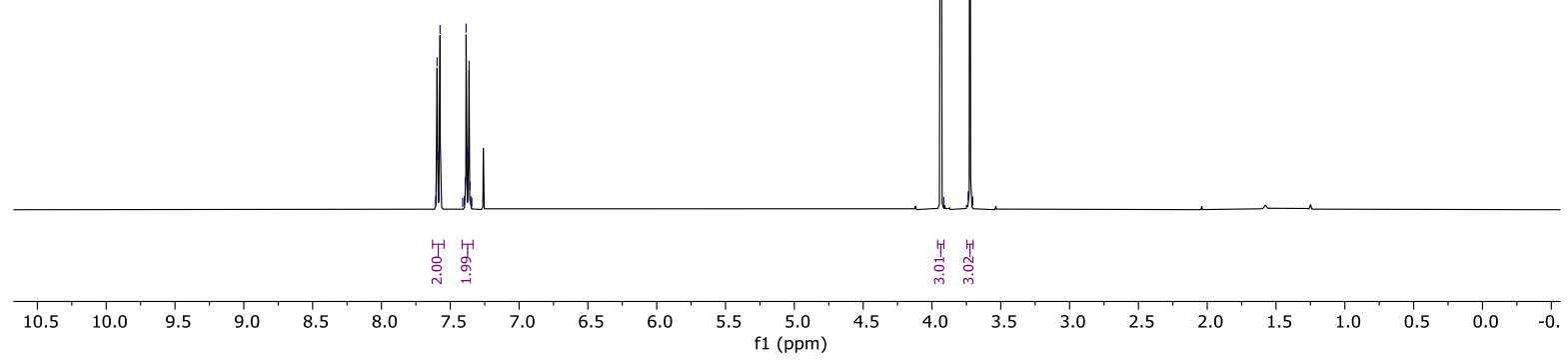<smiles>COC(=O)C(C#N)=C(C#N)c1ccc(Br)cc1</smiles>

${ }^{13} \mathrm{C}$ NMR $101 \mathrm{MHz} \mathrm{CDCl}_{3}$

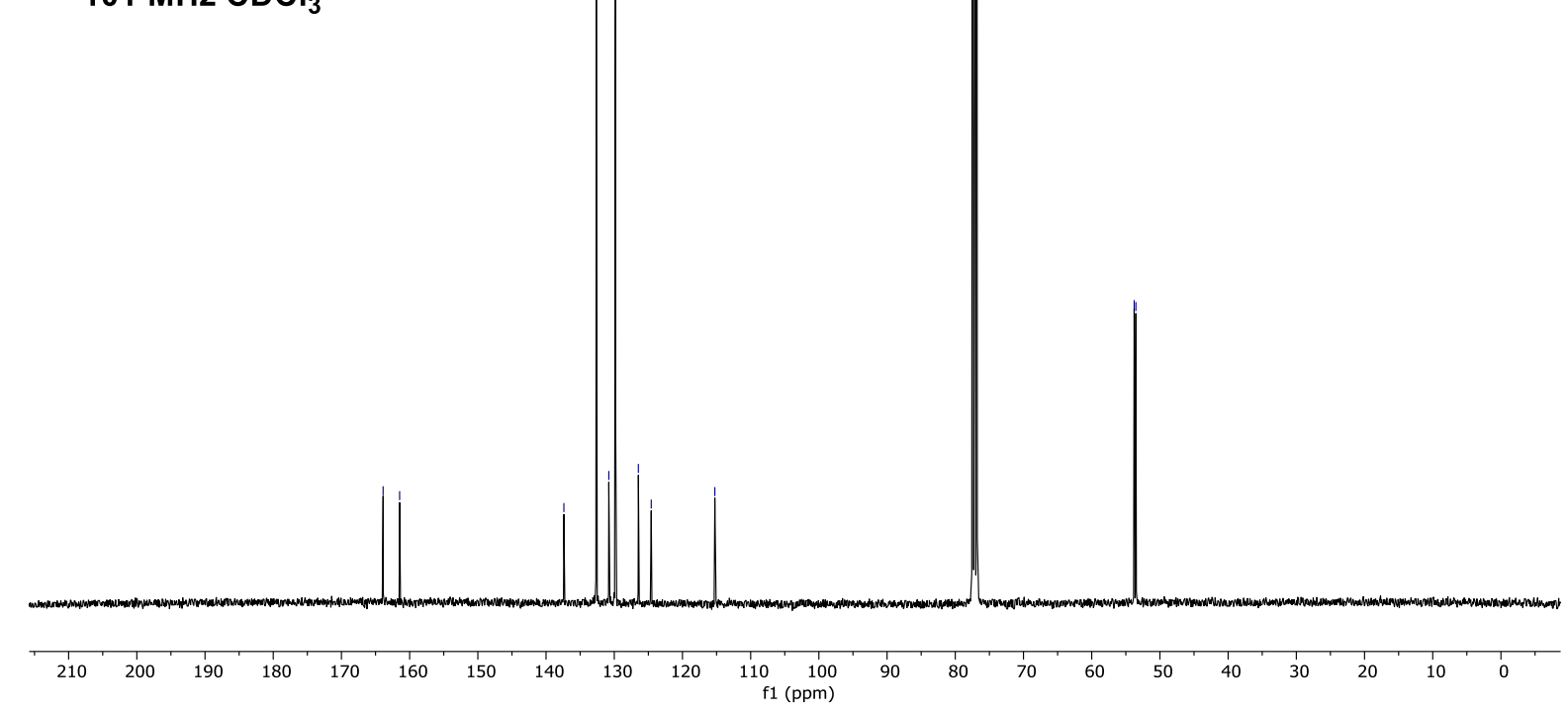



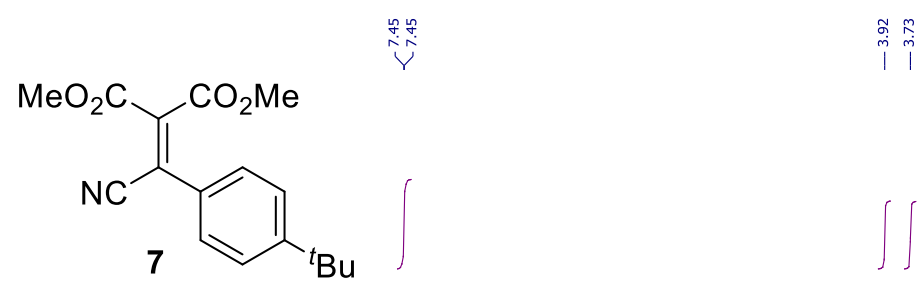

${ }^{1} \mathrm{H}$ NMR

$400 \mathrm{MHz} \mathrm{CDCl}_{3}$
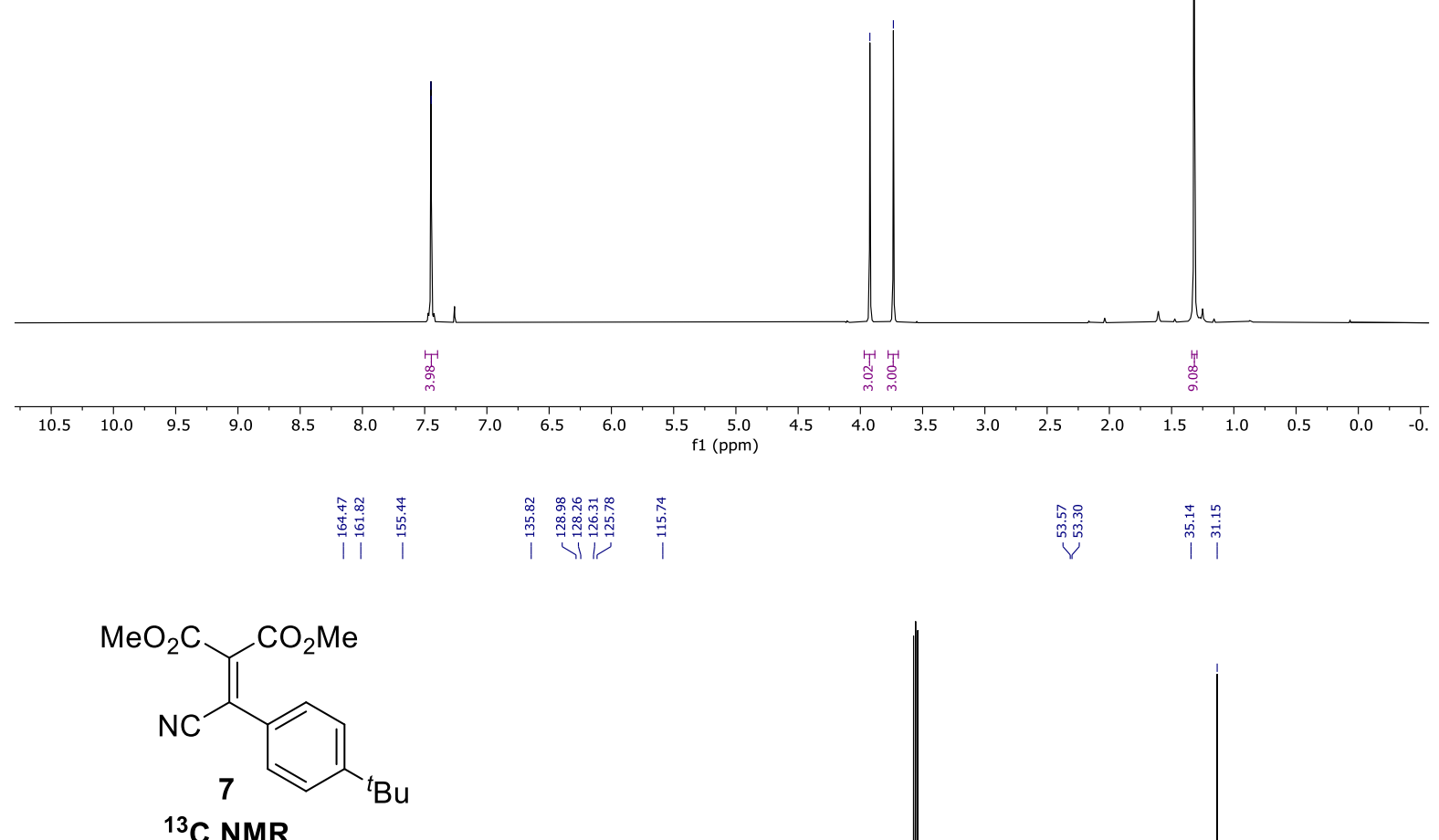

$101 \mathrm{MHz}^{\mathrm{CDCl}}{ }_{3}$

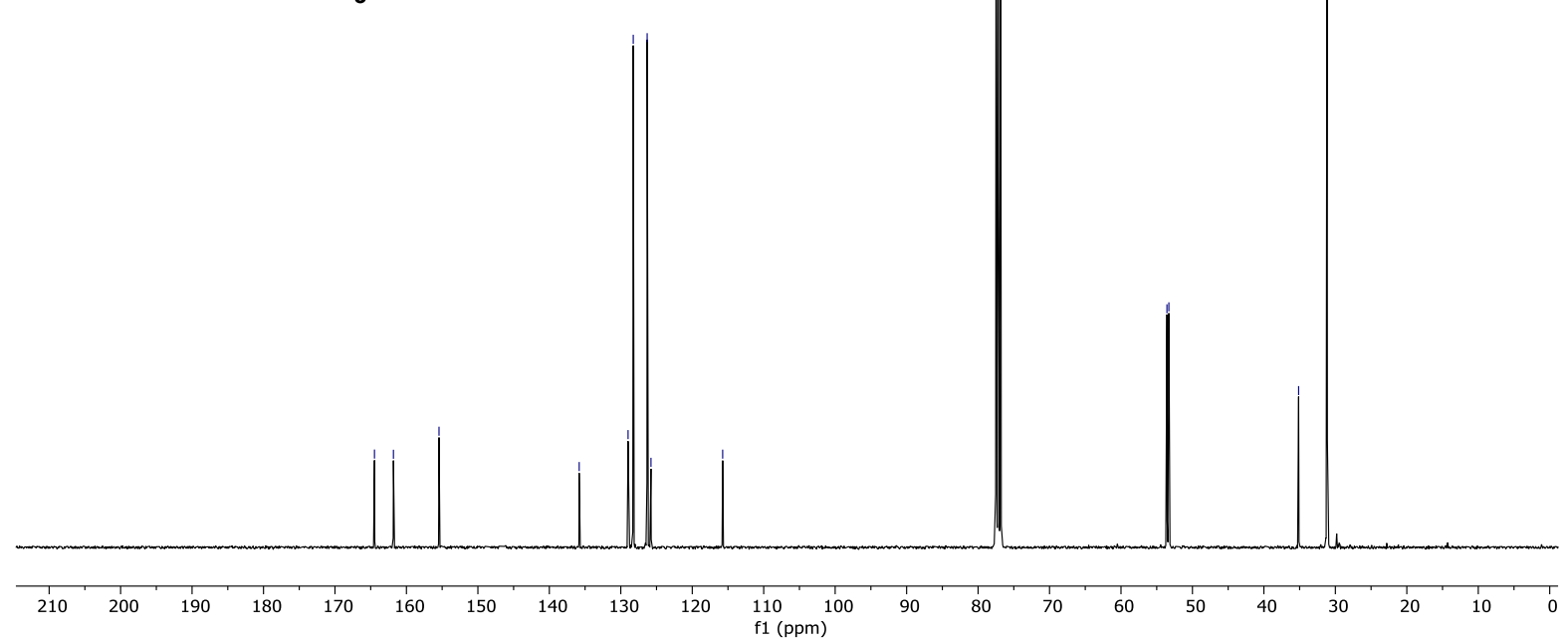




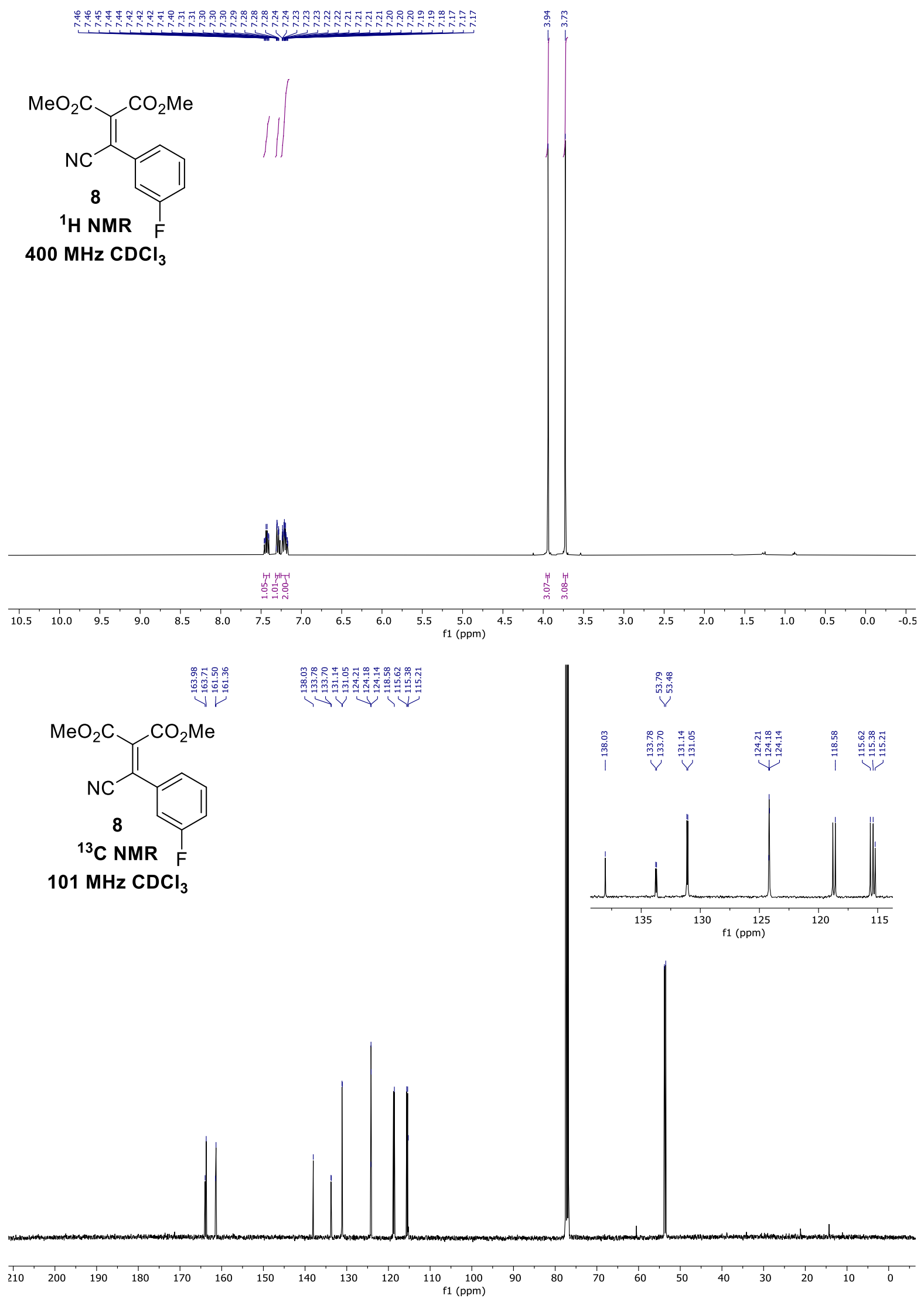




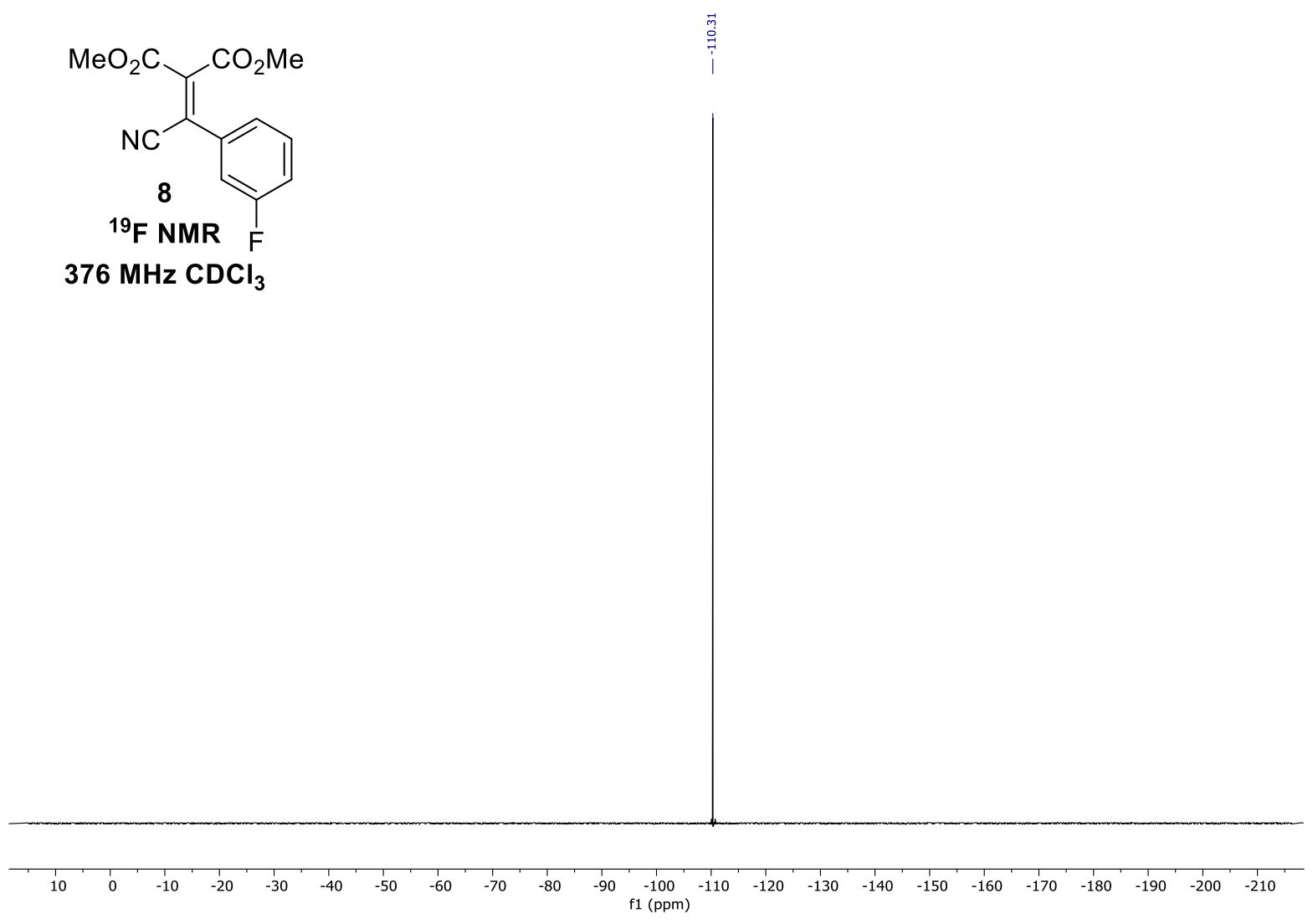



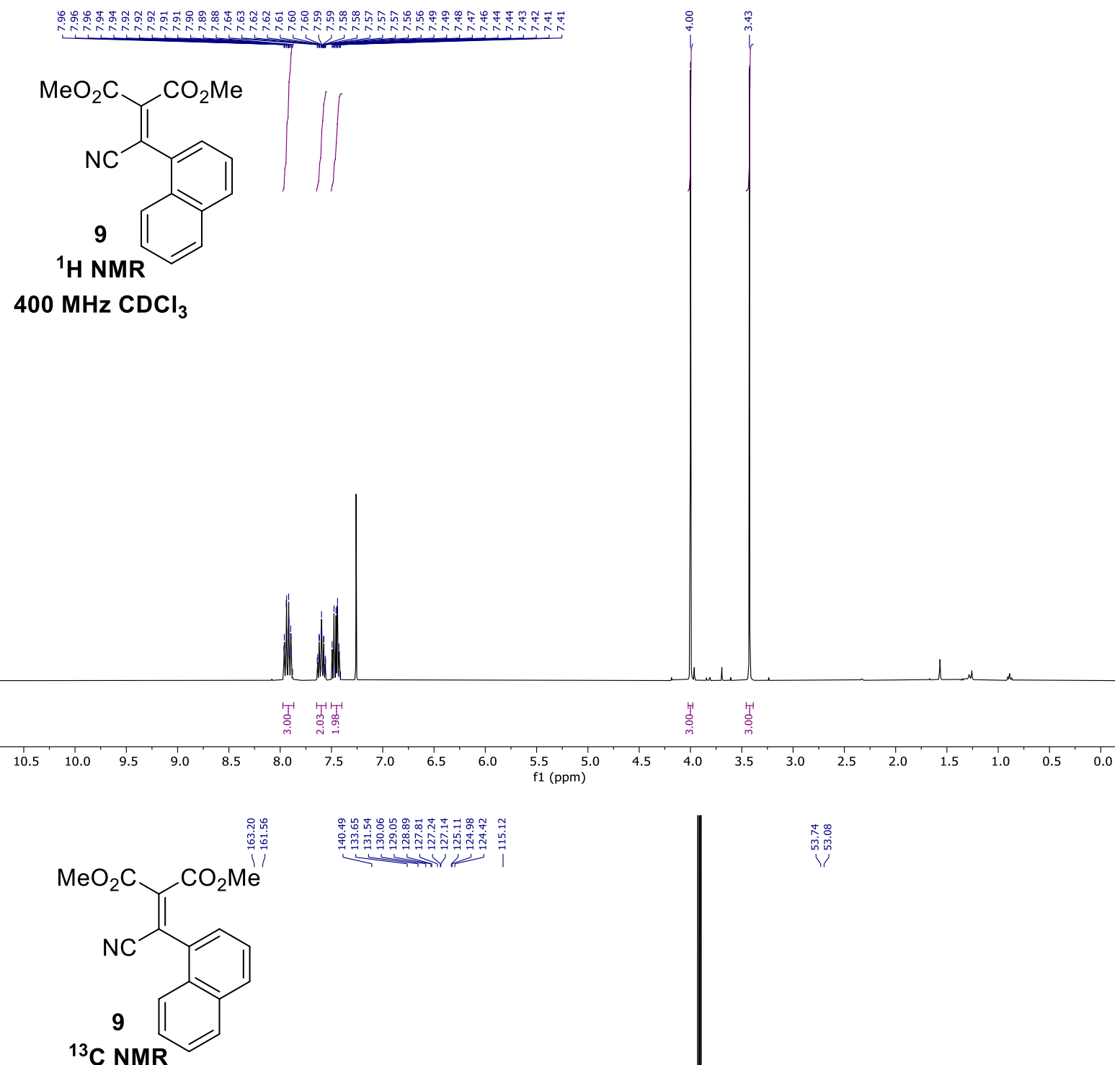

$101 \mathrm{MHz} \mathrm{CDCl}_{3}$

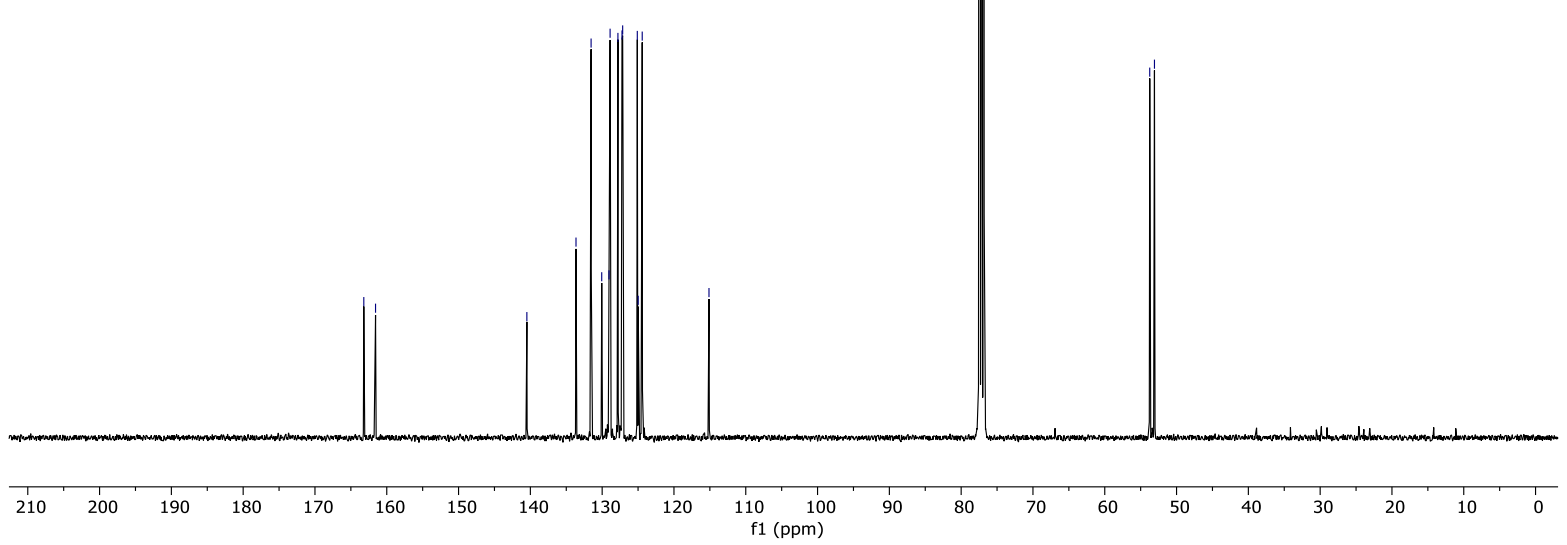



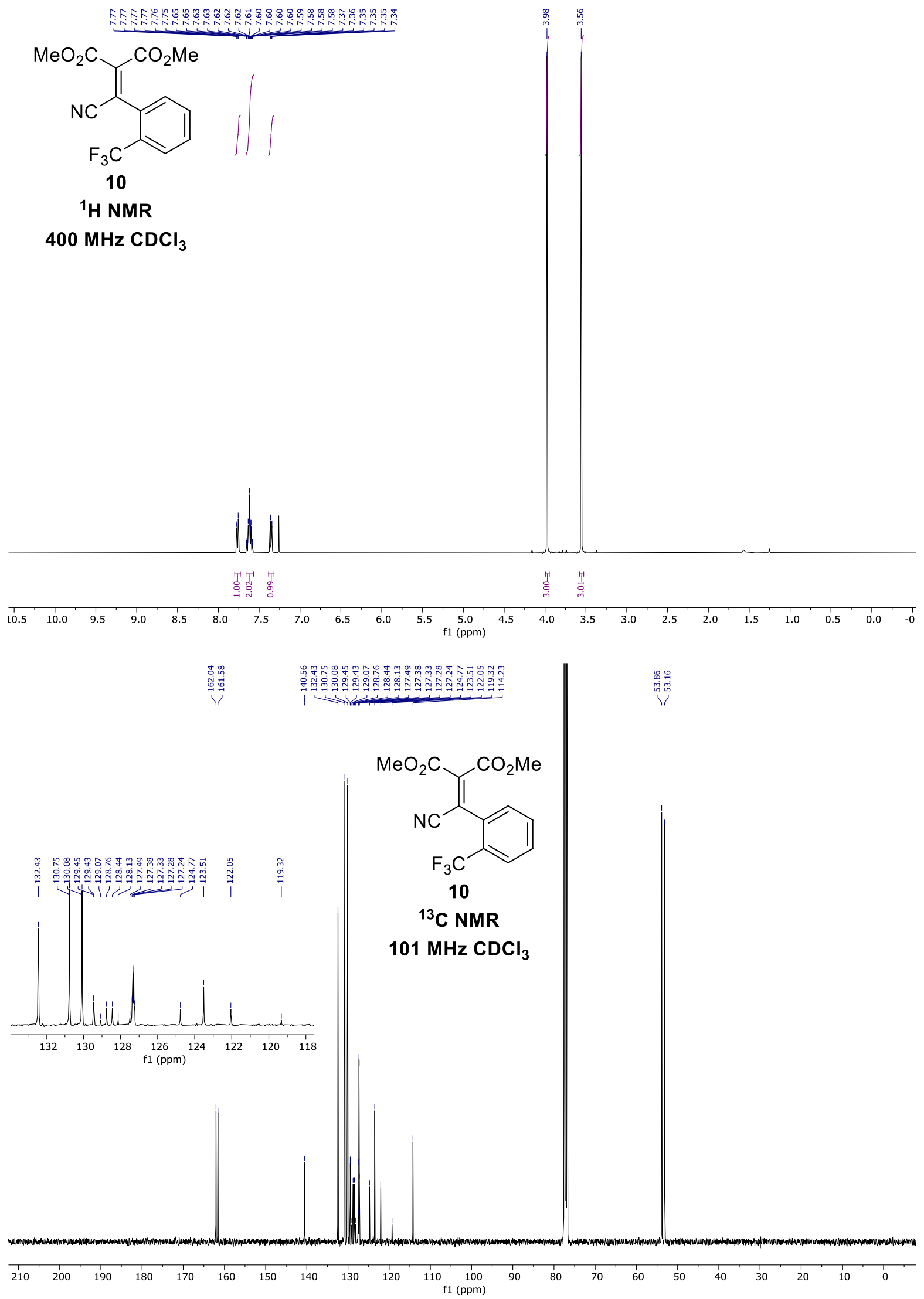


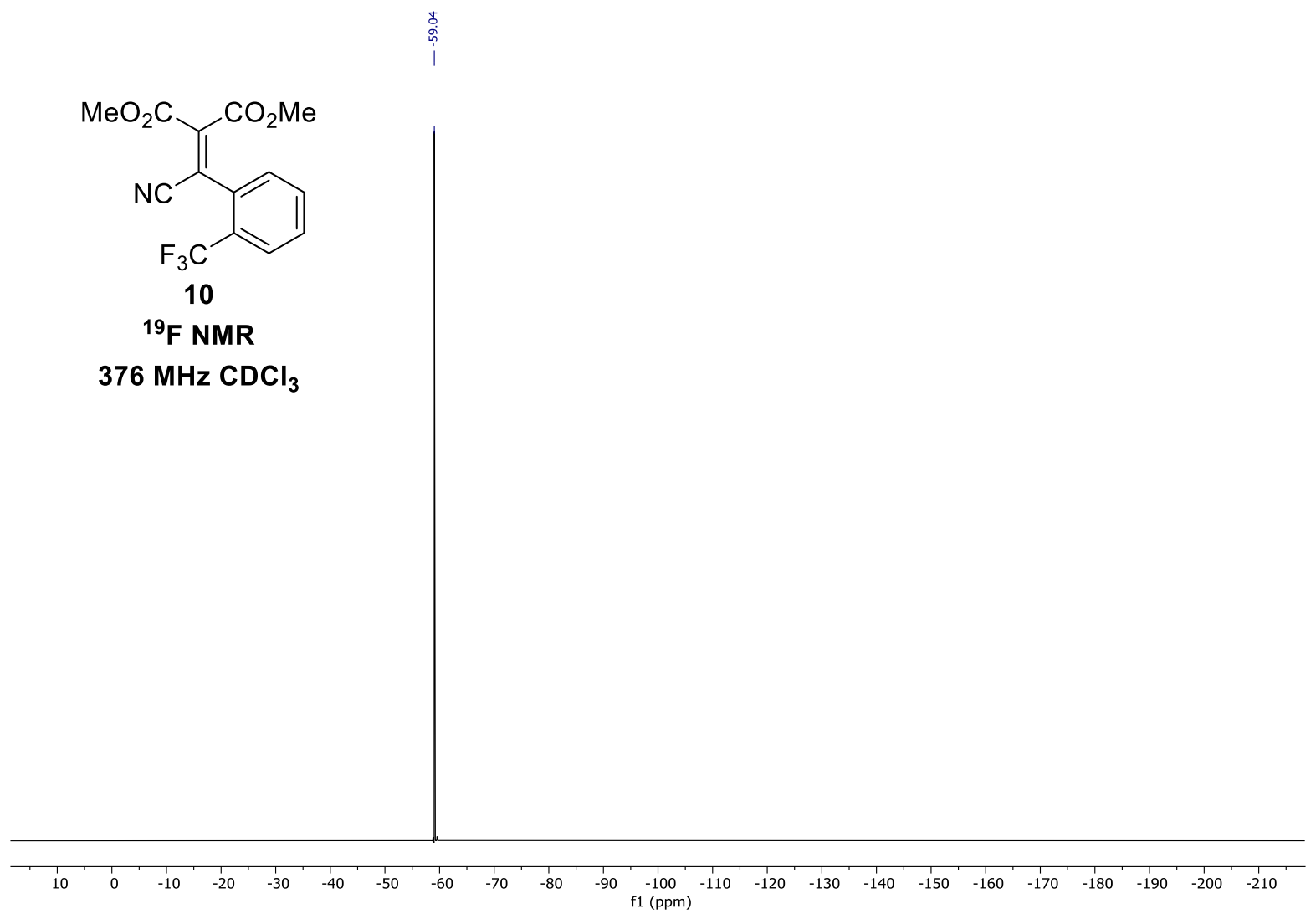



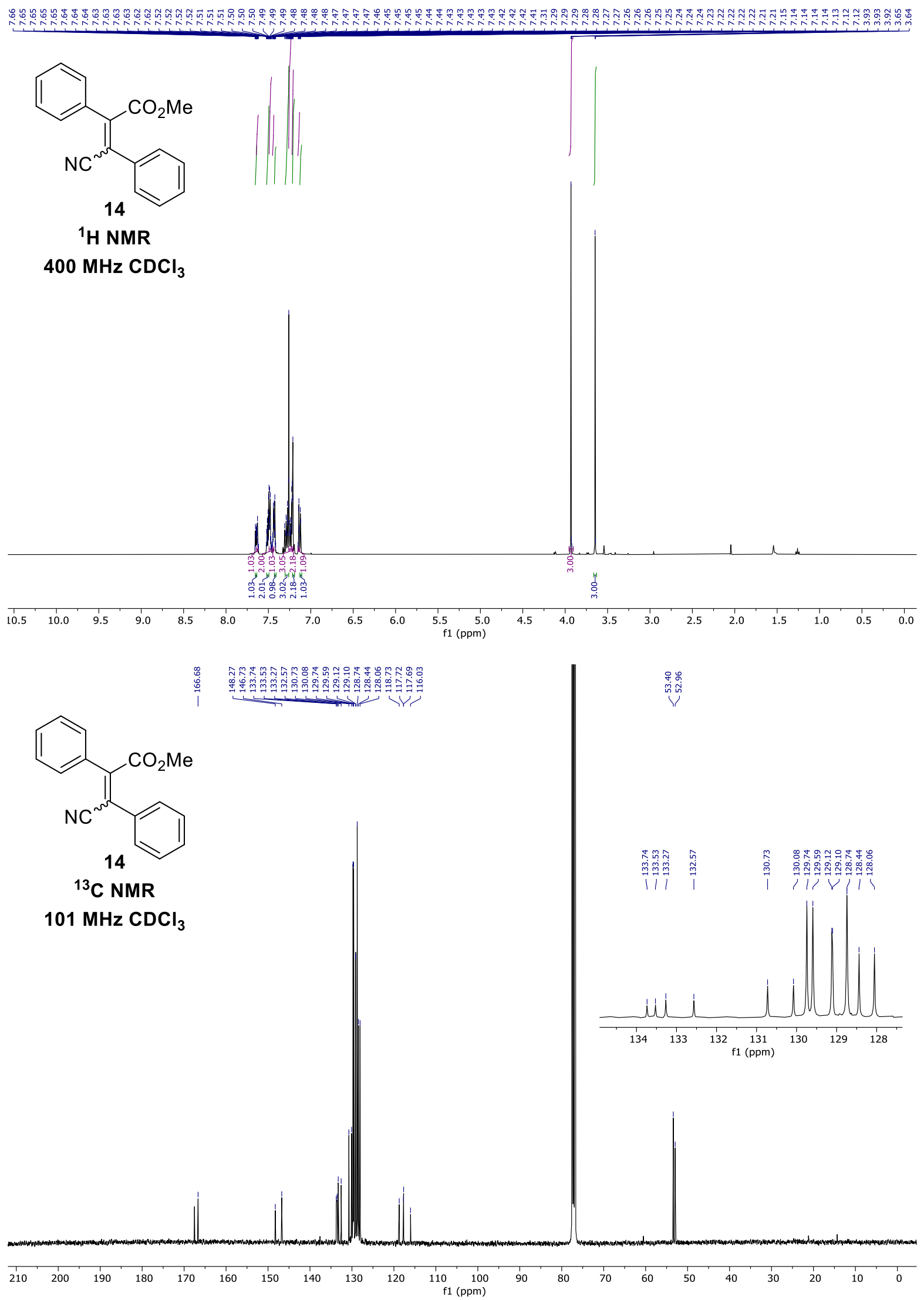

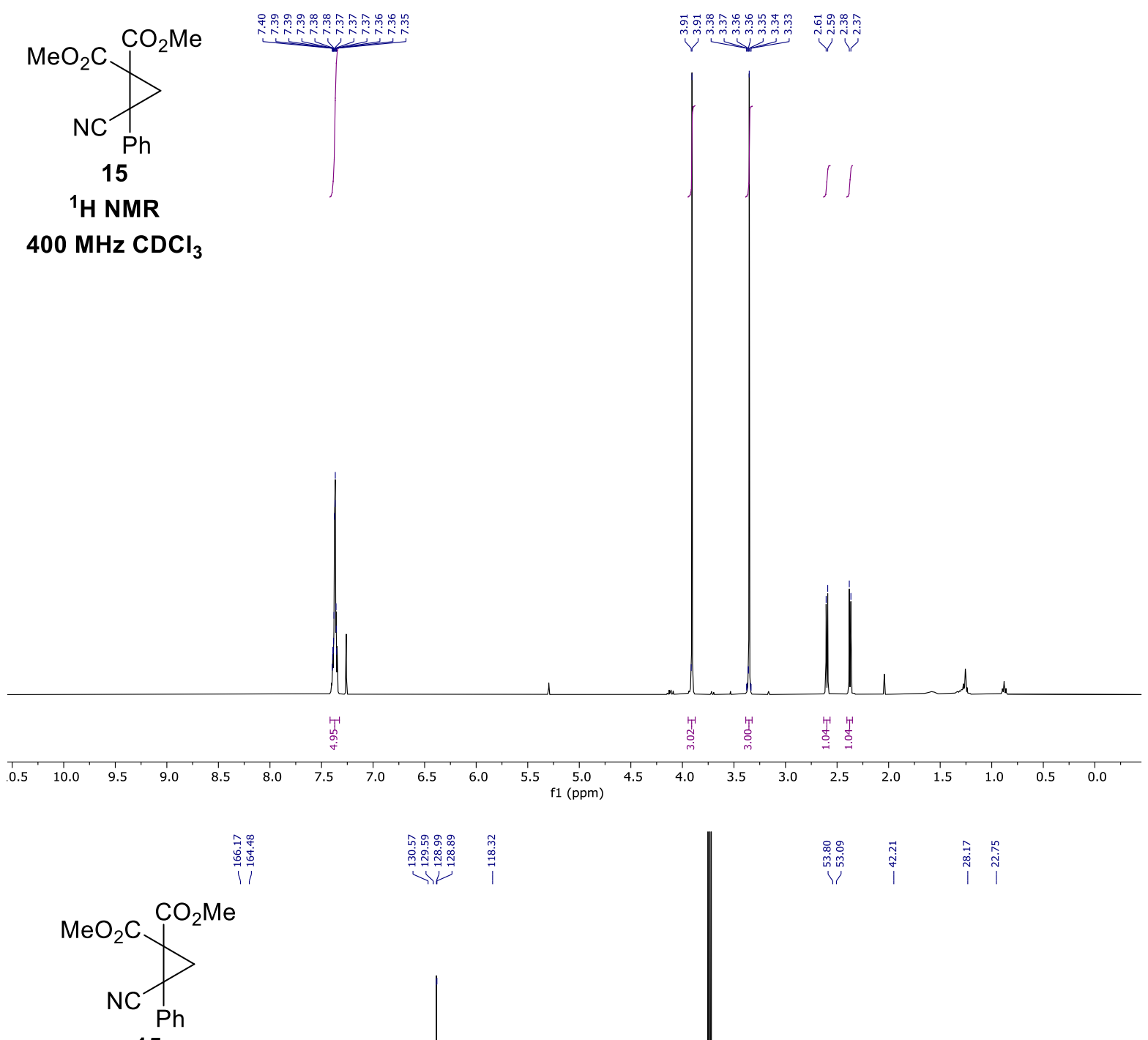

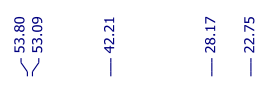

15

${ }^{13}$ C NMR

$101 \mathrm{MHz}^{\mathrm{CDCl}} \mathrm{CDC}_{3}$

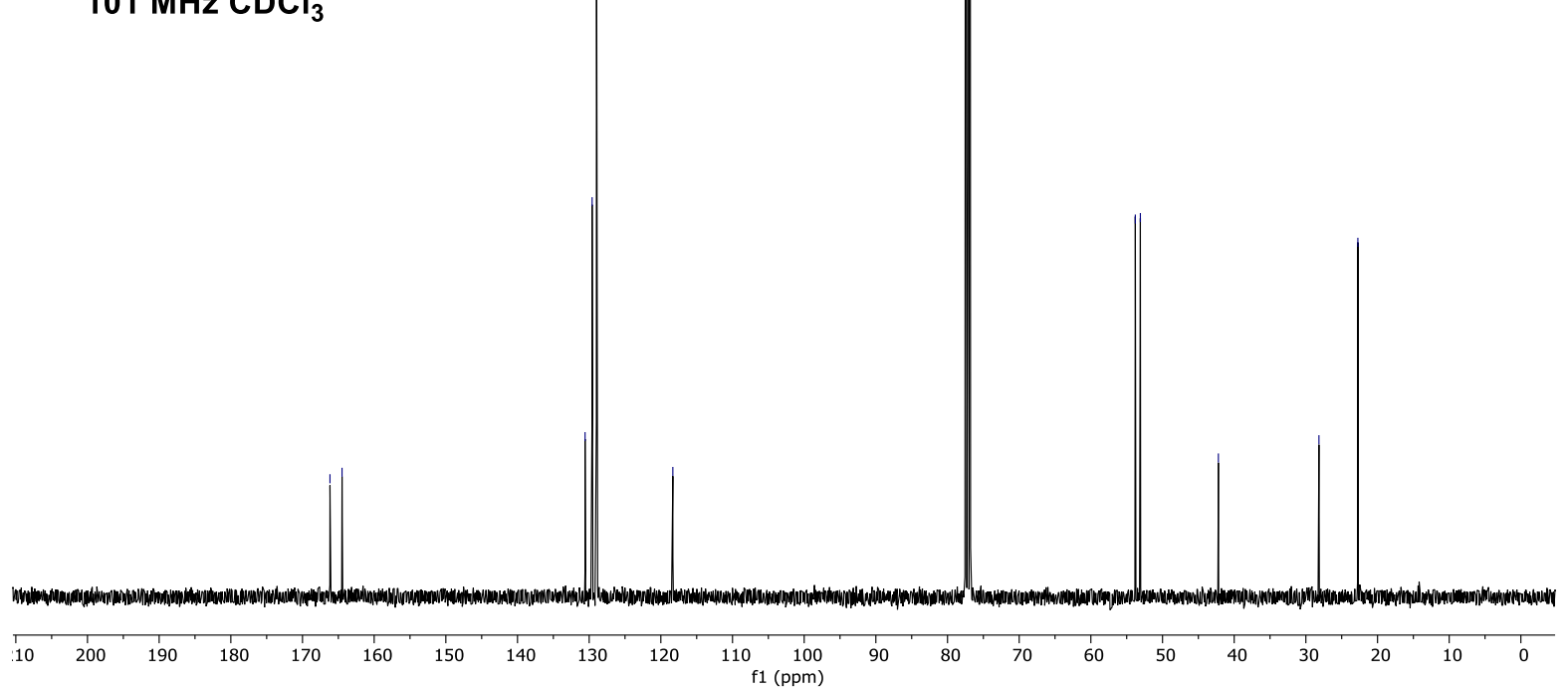




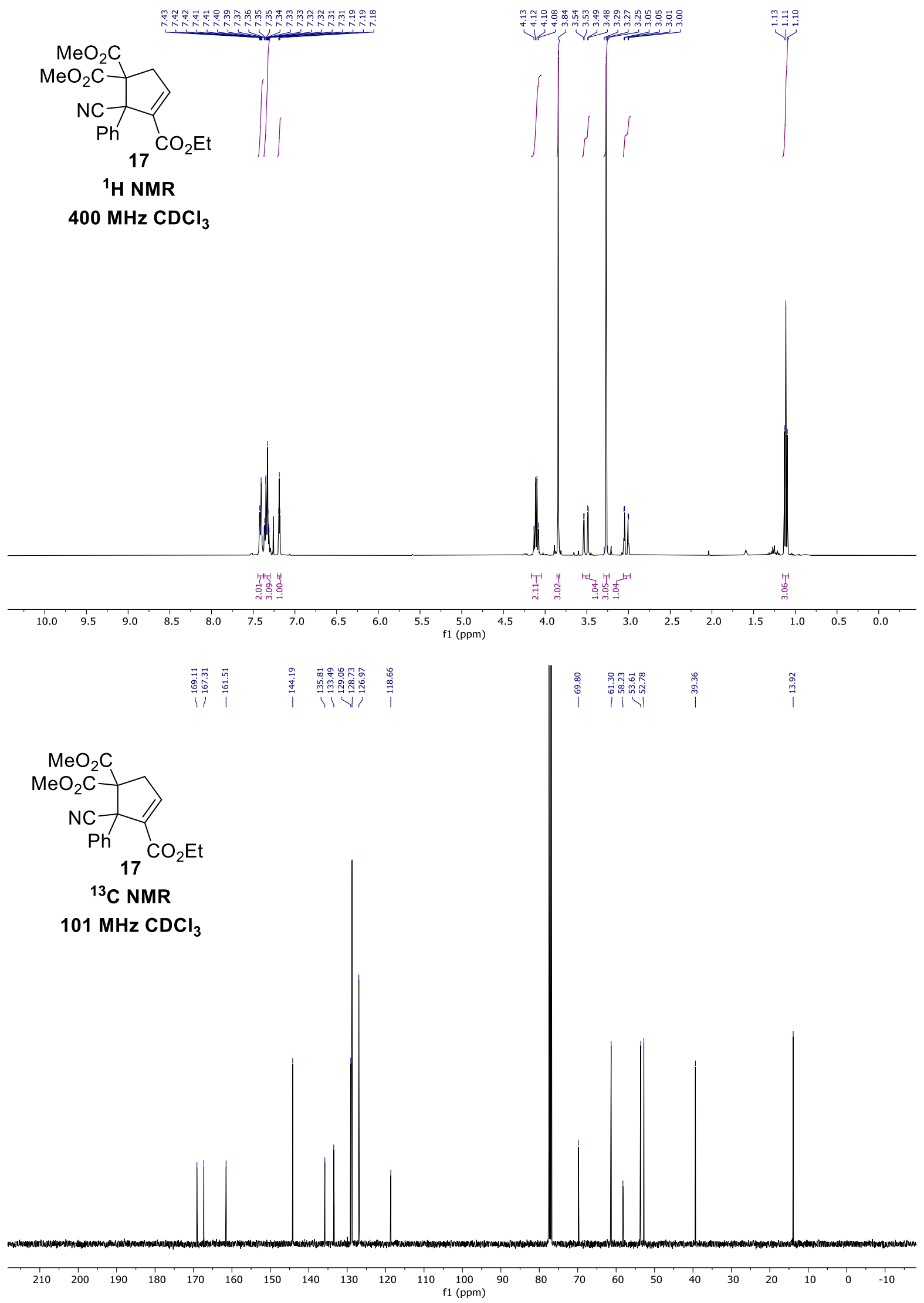



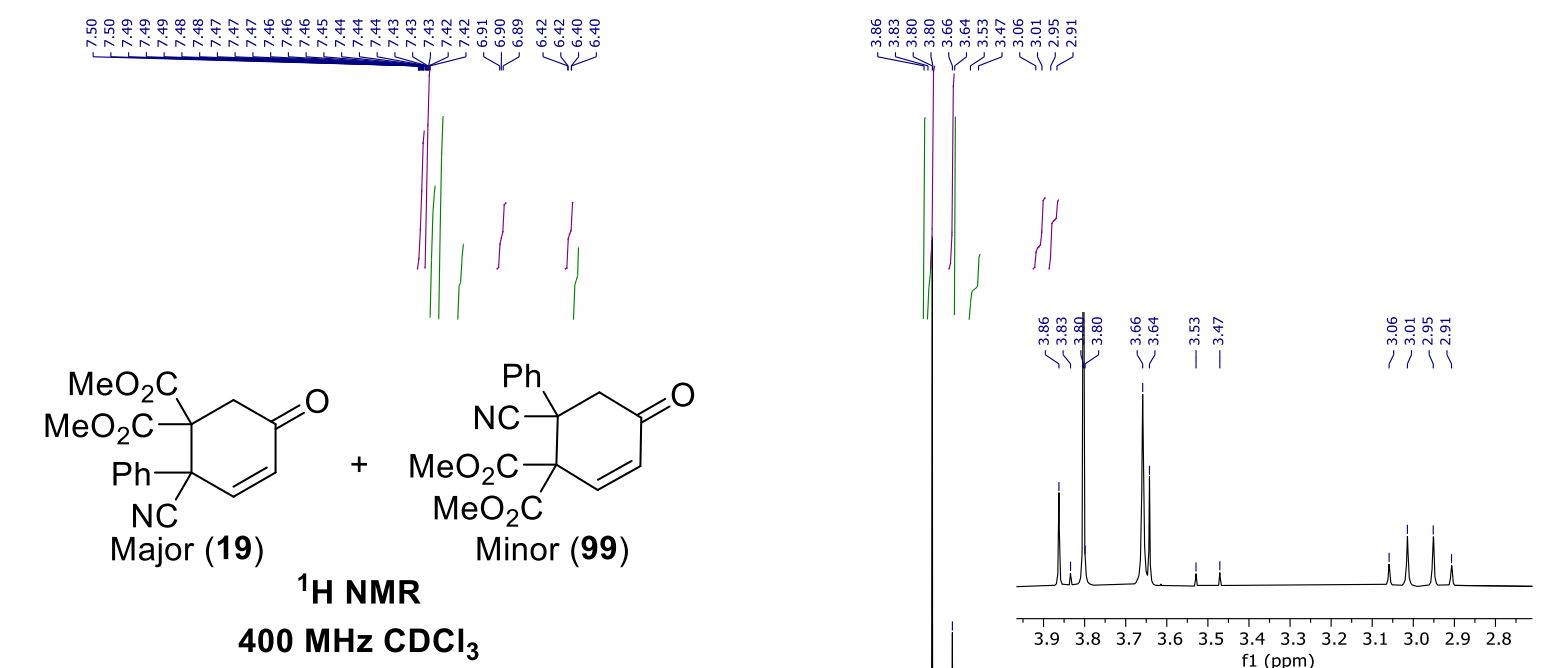

$00 \mathrm{MHz} \mathrm{CDCl}_{3}$
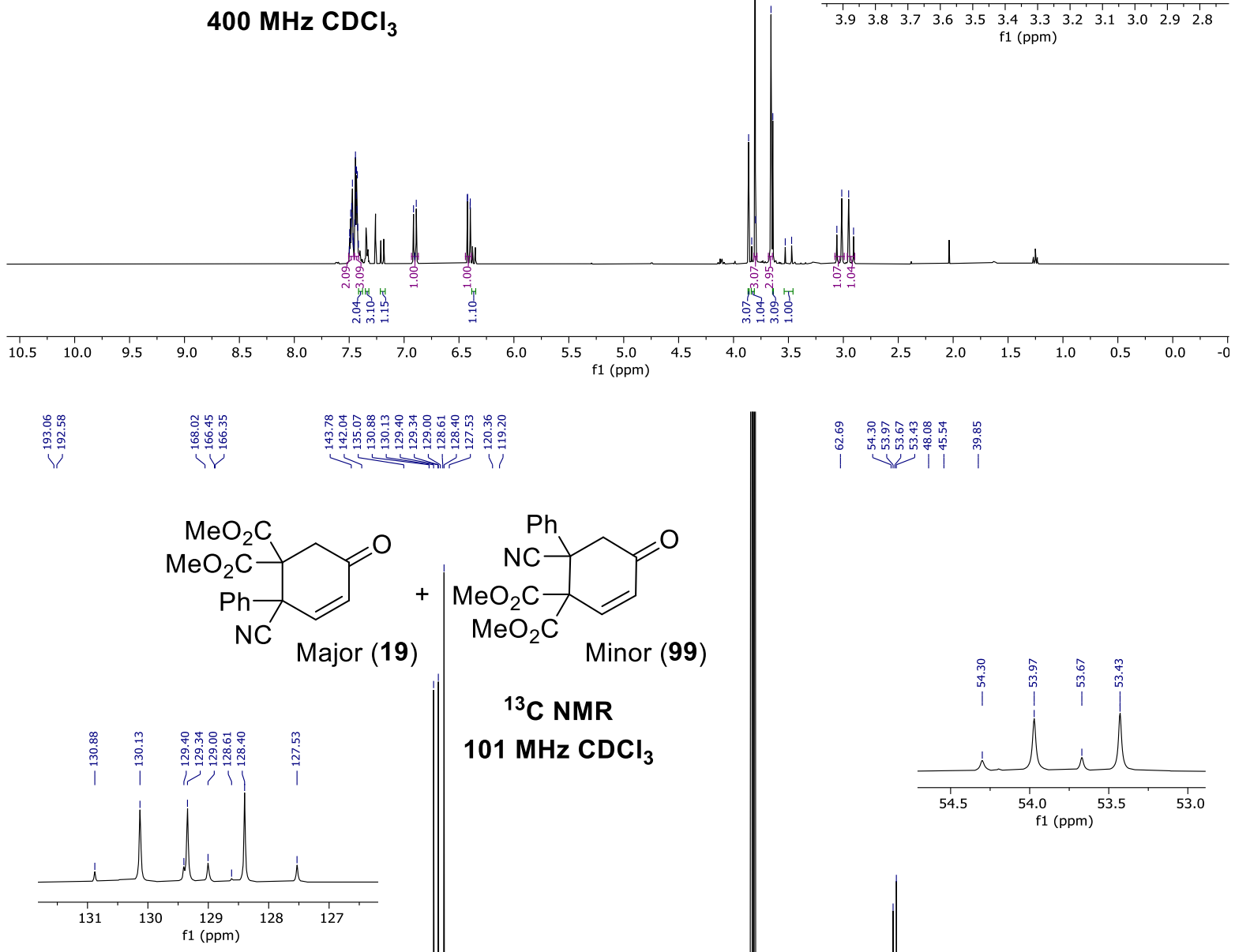

${ }^{13} \mathrm{C}$ NMR $101 \mathrm{MHz} \mathrm{CDCl}_{3}$
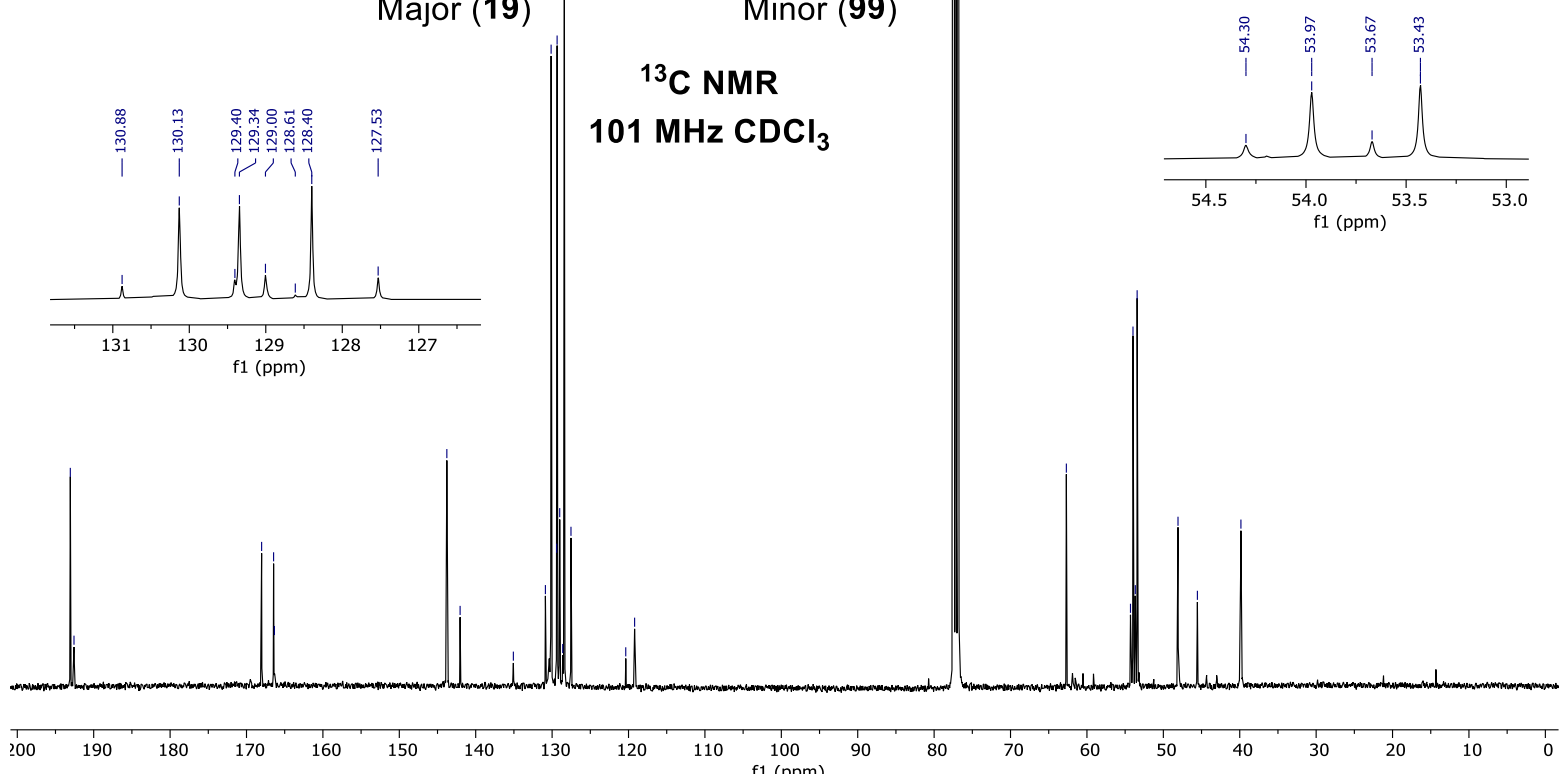

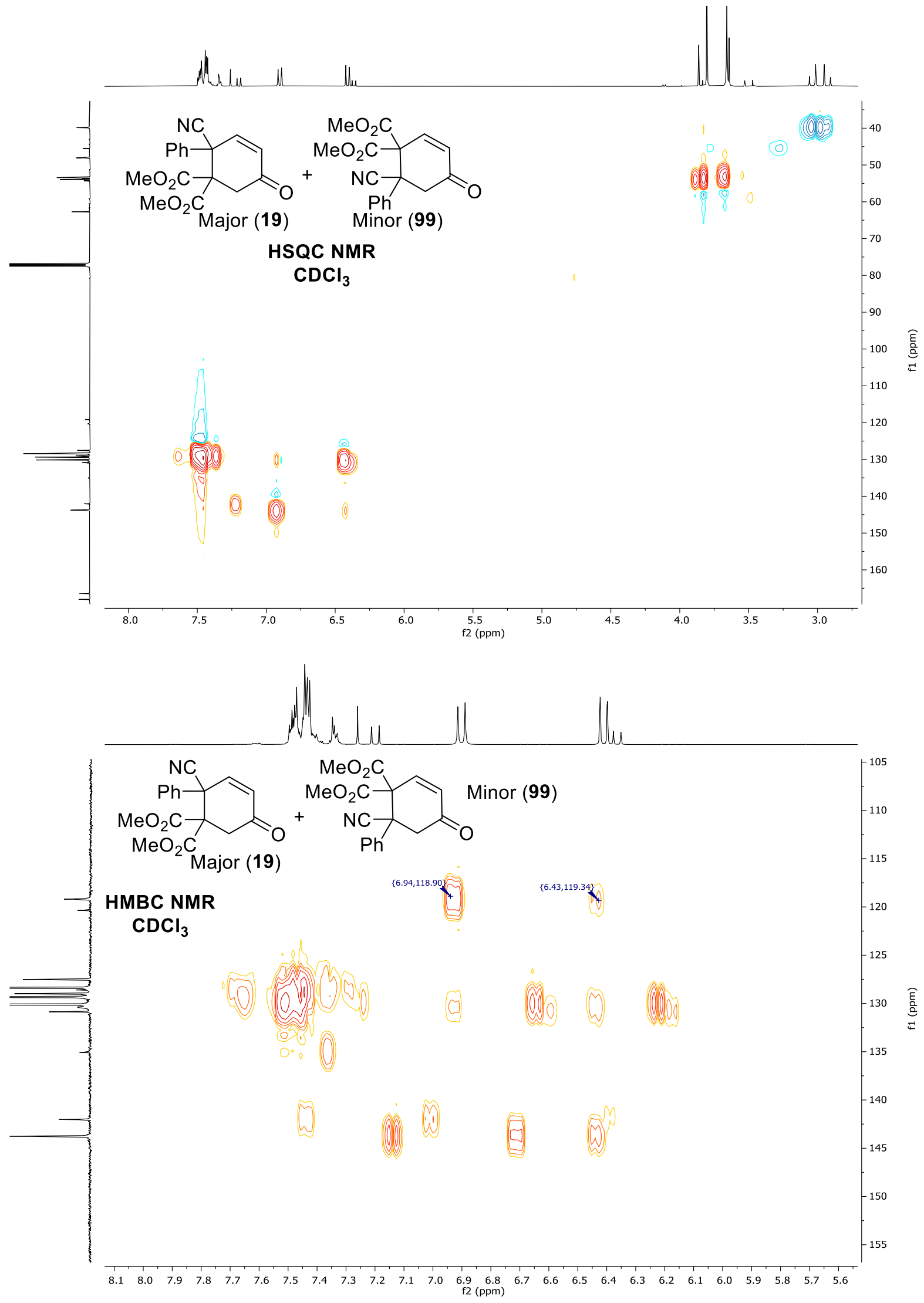

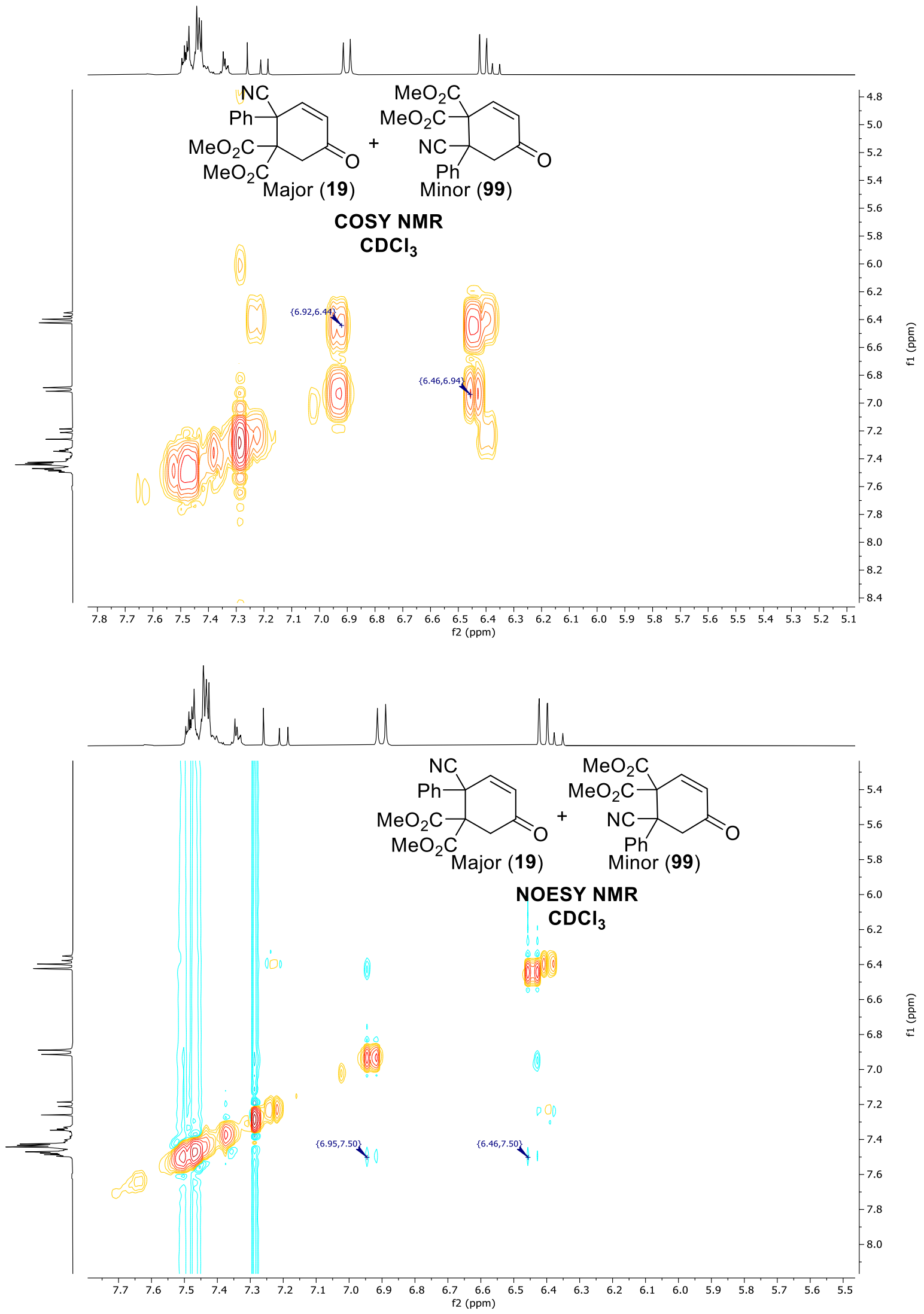


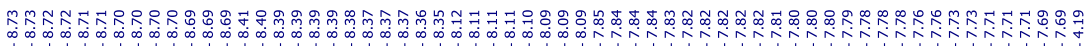

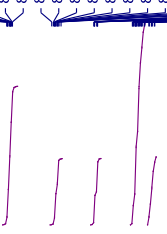<smiles>COC(=O)c1c(C#N)c2ccccc2c2ccccc12</smiles>

20

${ }^{1} \mathrm{H}$ NMR $400 \mathrm{MHz} \mathrm{CDCl}_{3}$

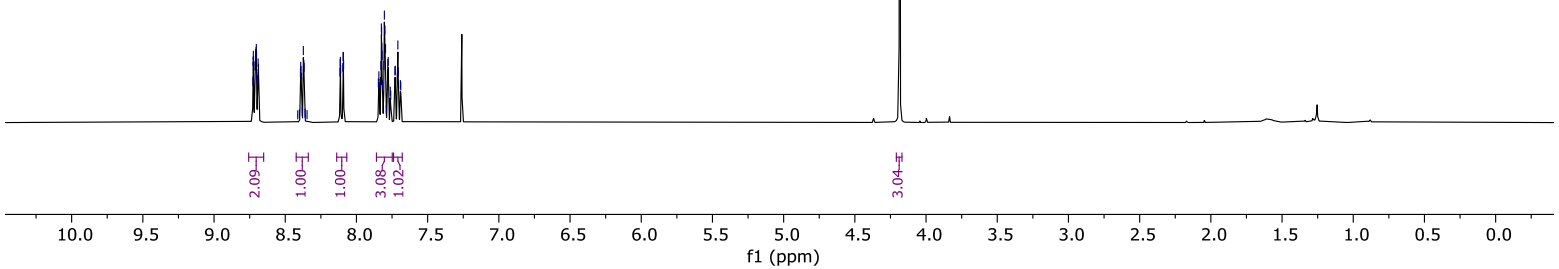<smiles>CC(=O)c1c(C#N)c2ccccc2c2ccccc12</smiles>

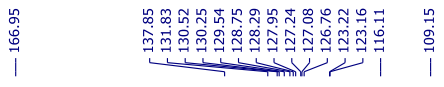

${ }^{13} \mathrm{C}$ NMR

$101 \mathrm{MHz} \mathrm{CDCl}_{3}$

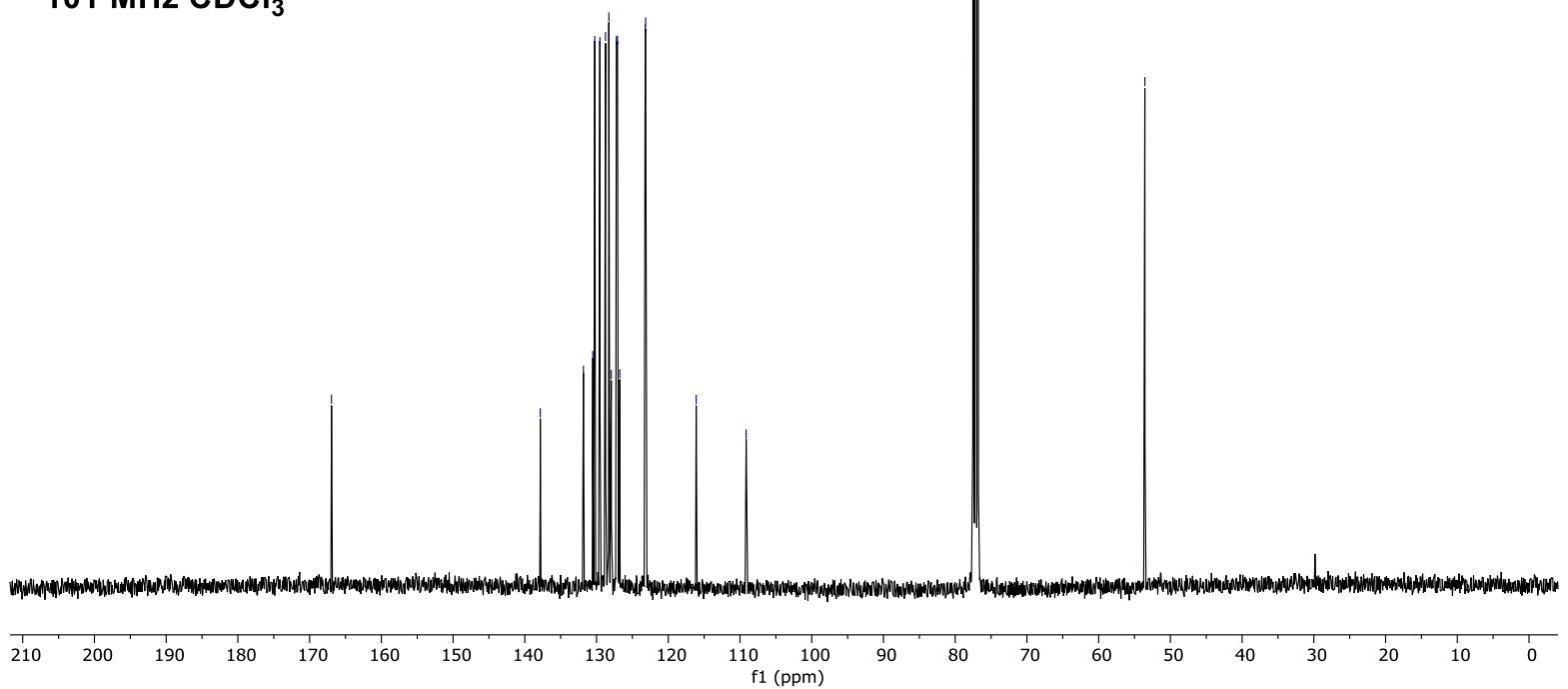



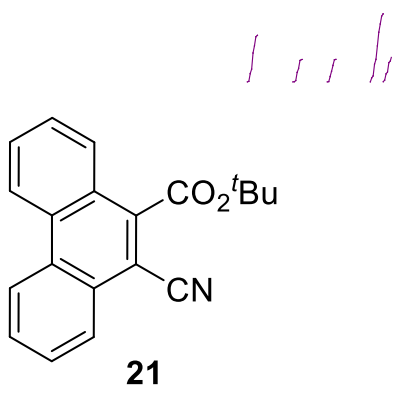

${ }^{1} \mathrm{H}$ NMR

$400 \mathrm{MHz} \mathrm{CDCl}_{3}$
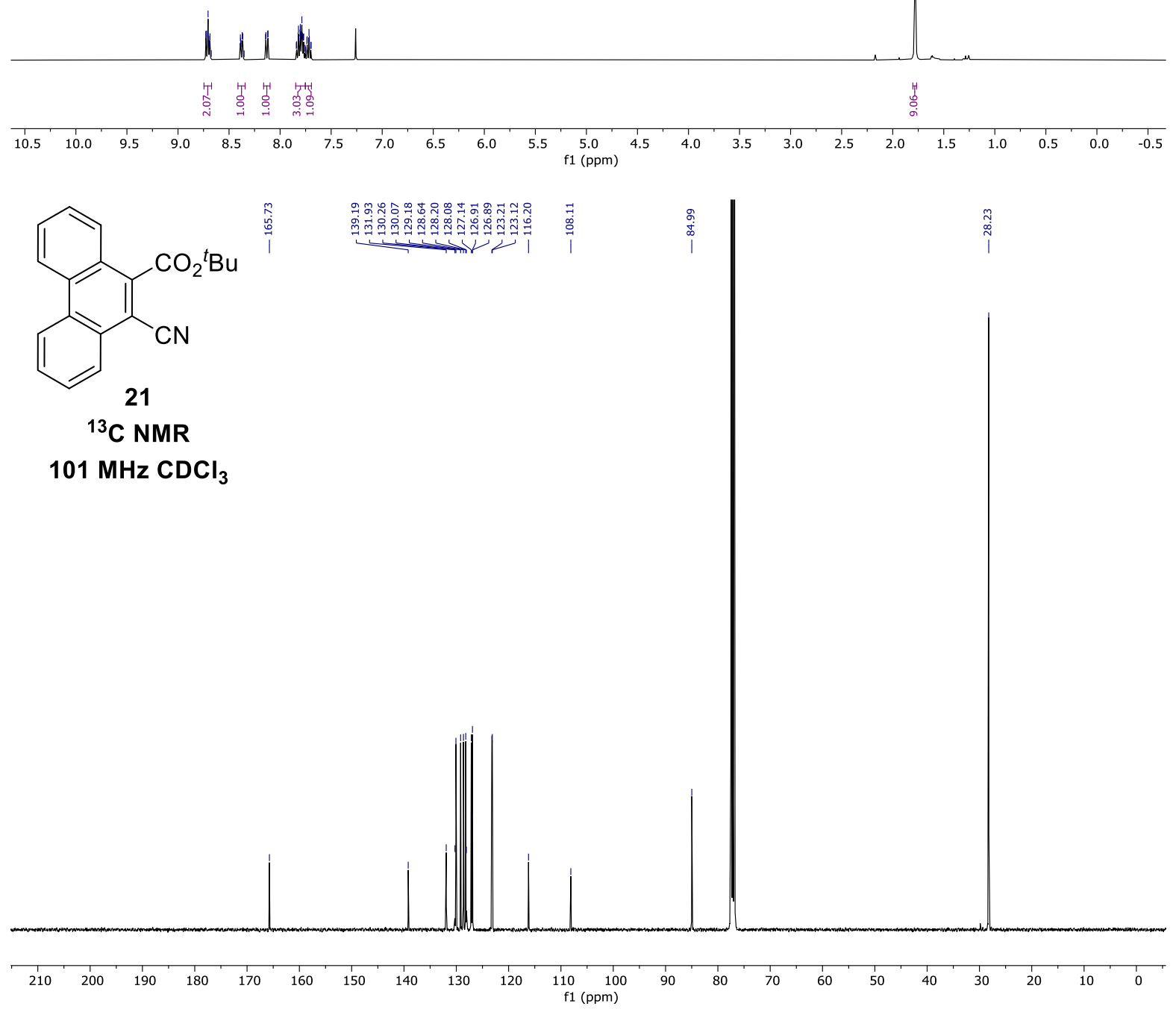


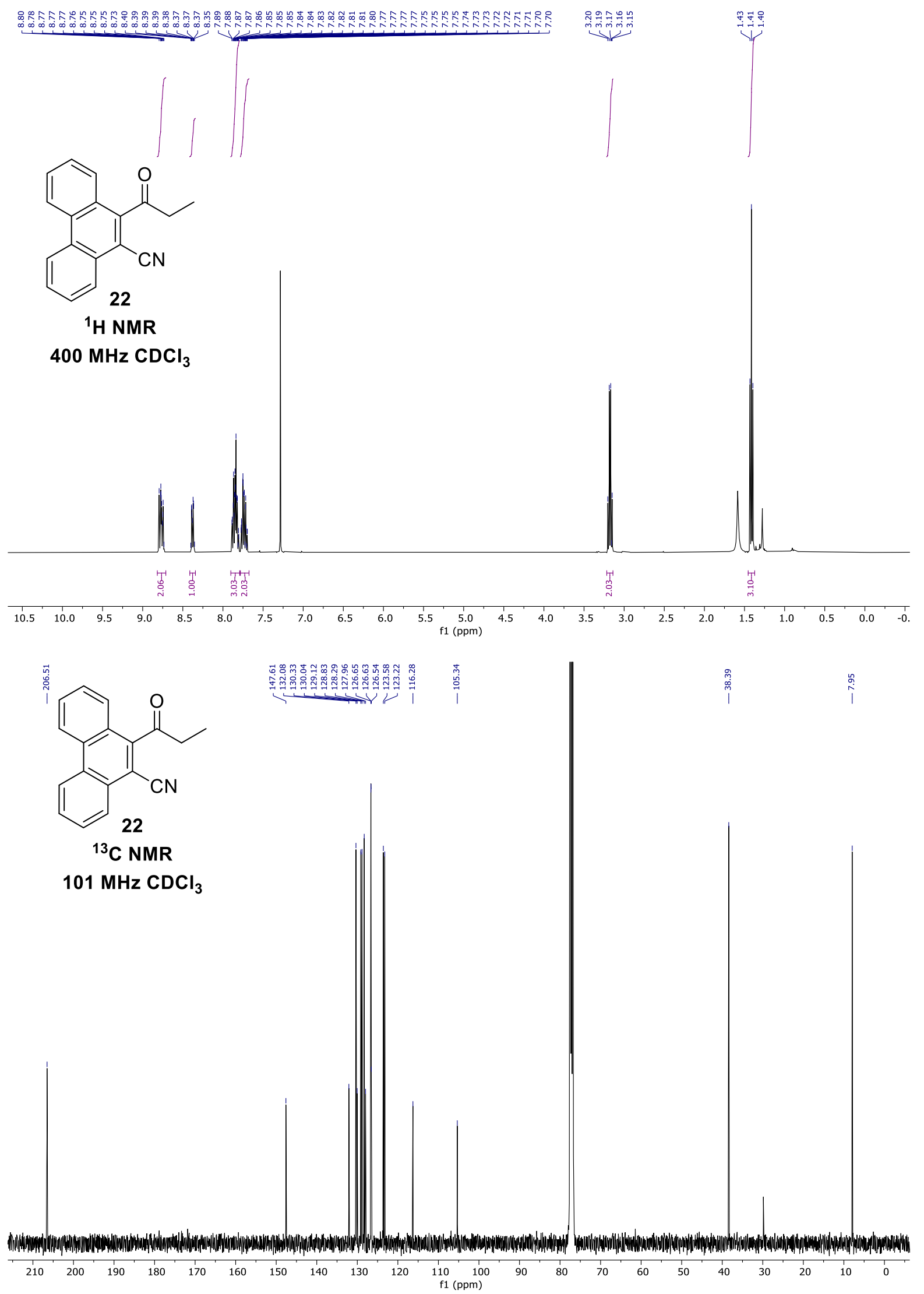




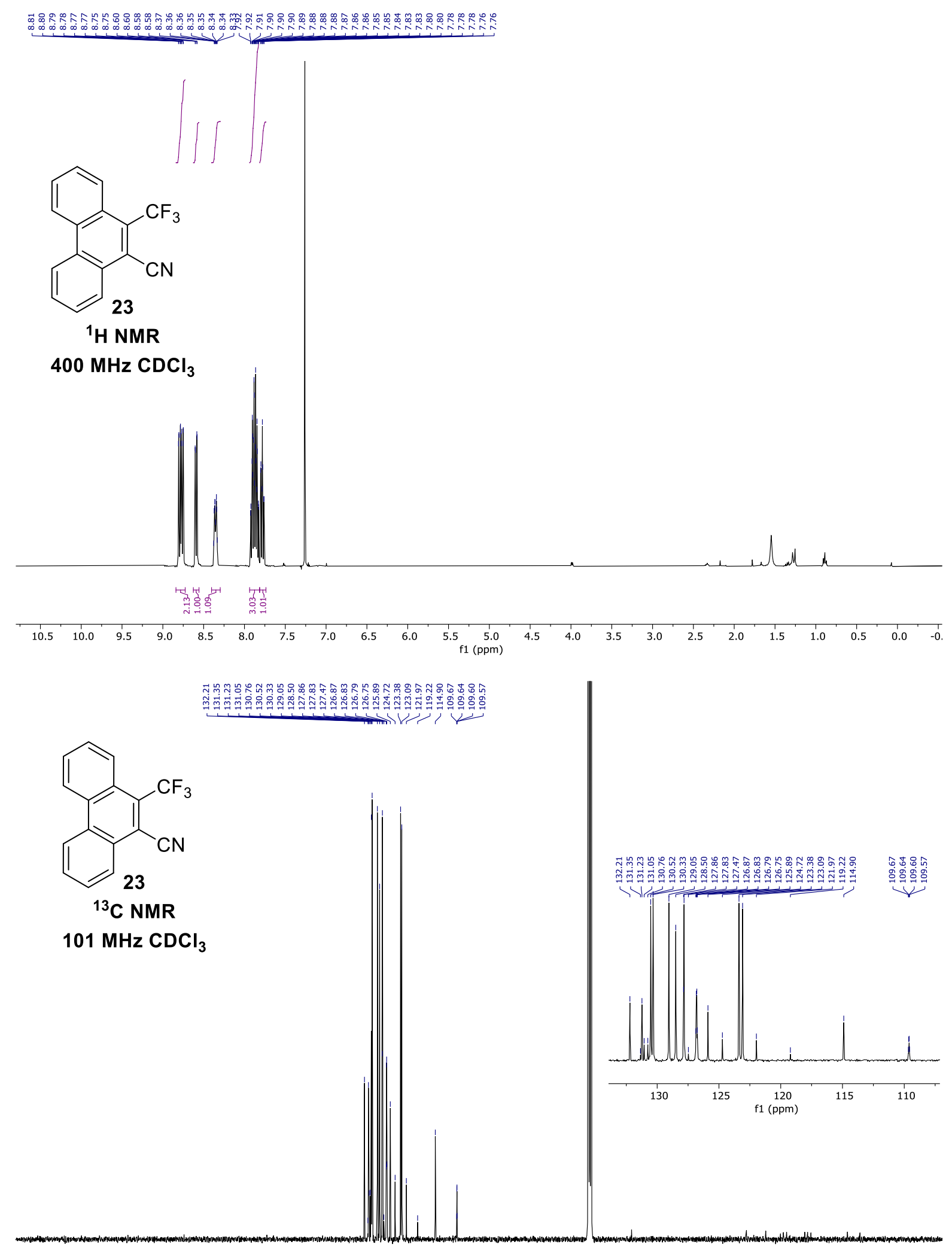

(1)

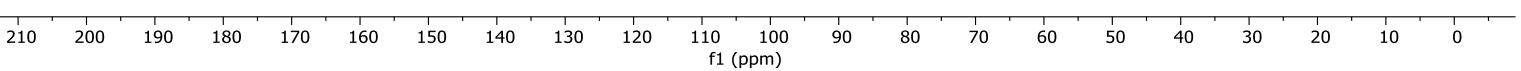


<smiles>N#Cc1c(C(F)(F)F)c2ccccc2c2ccccc12</smiles>

${ }^{19} \mathrm{~F}$ NMR $376 \mathrm{MHz} \mathrm{CDCl}_{3}$

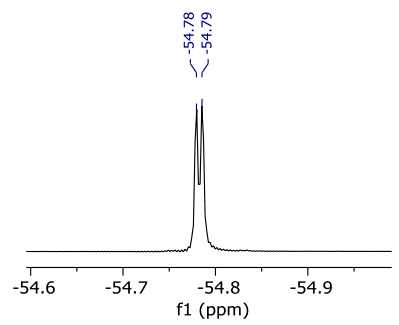

$\begin{array}{lllllllllllllllllllllll}10 & 0 & -10 & -20 & -30 & -40 & -50 & -60 & -70 & -80 & -90 & -100 & -110 & -120 & -130 & -140 & -150 & -160 & -170 & -180 & -190 & -200 & -210\end{array}$




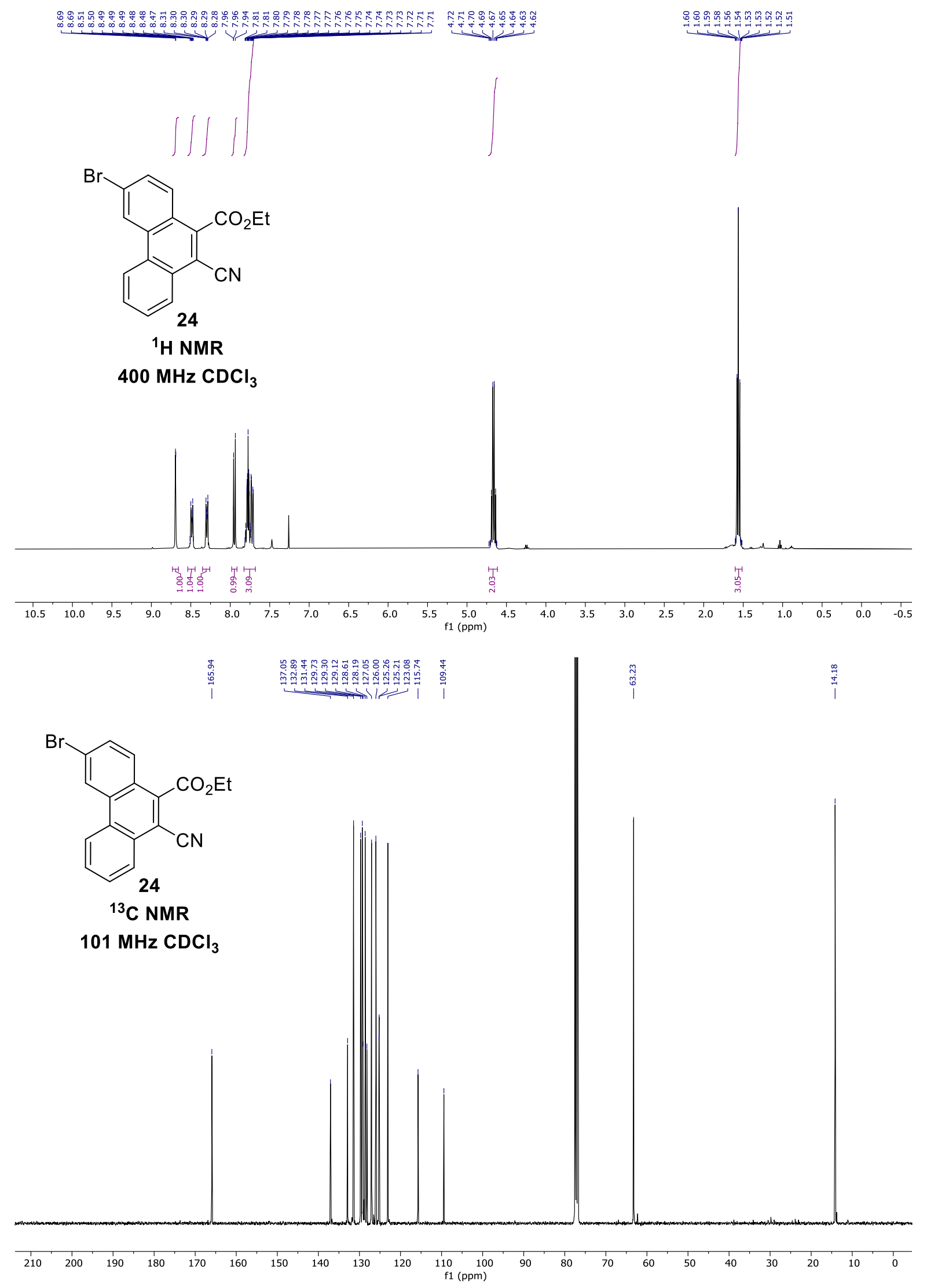




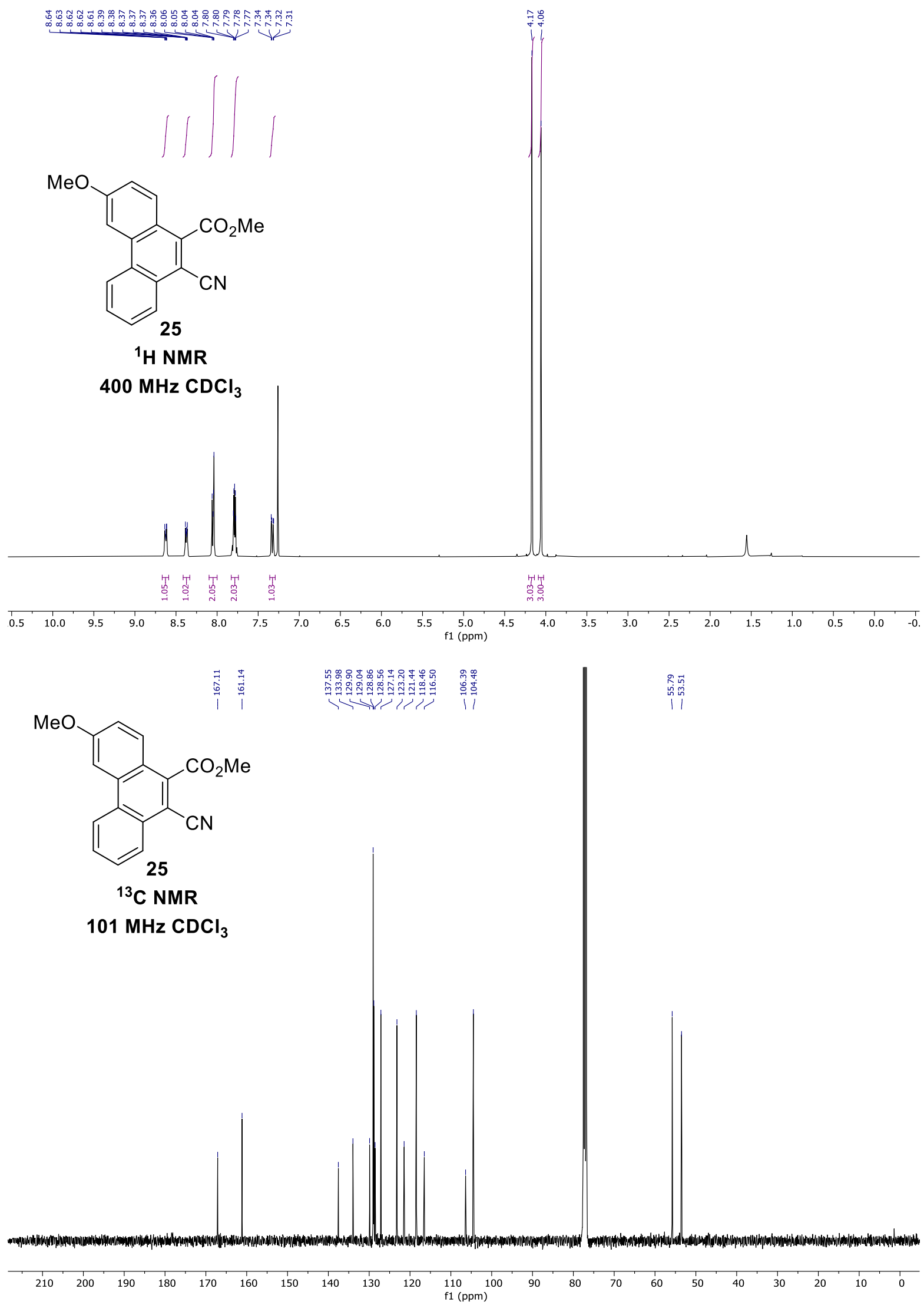




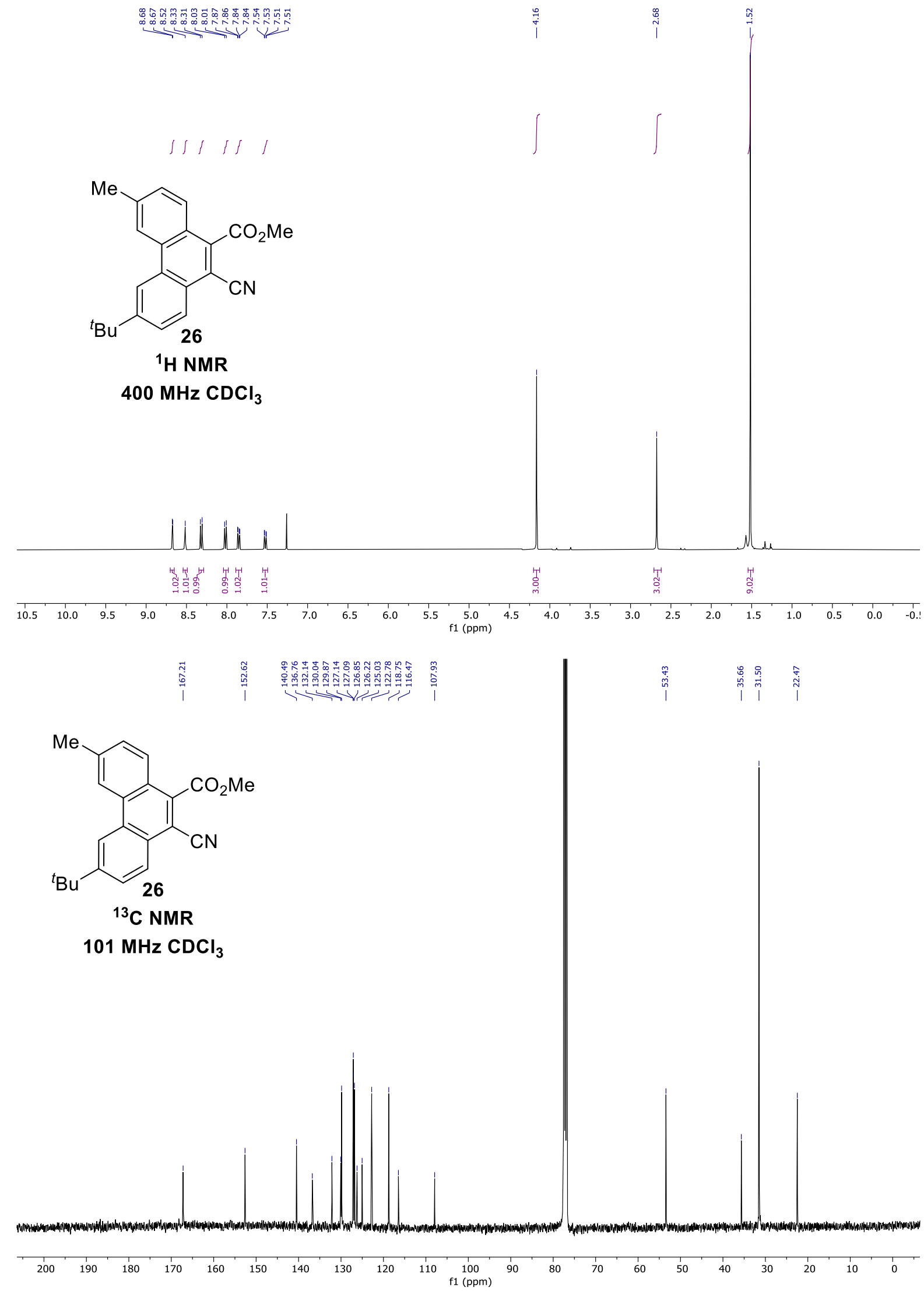




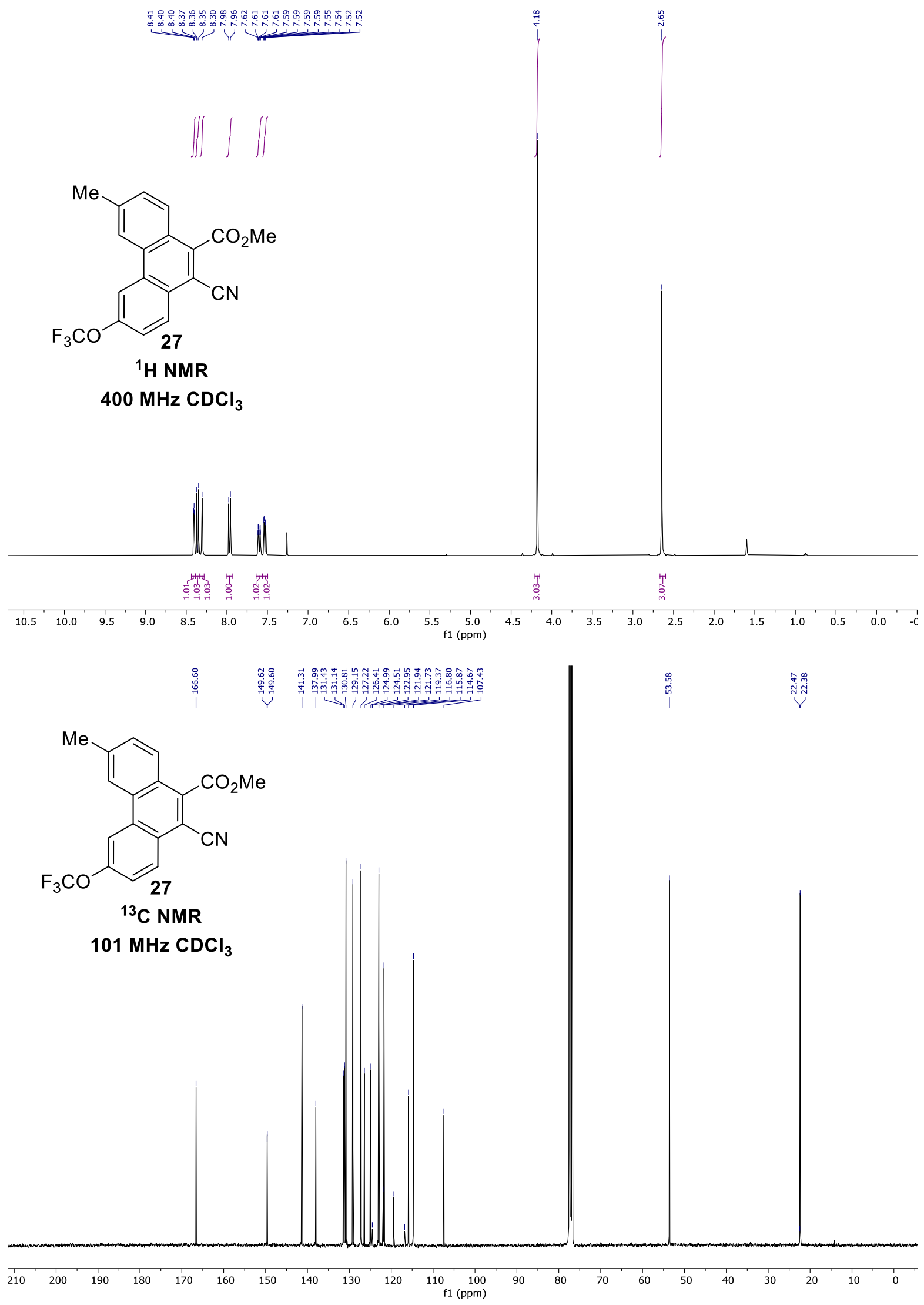




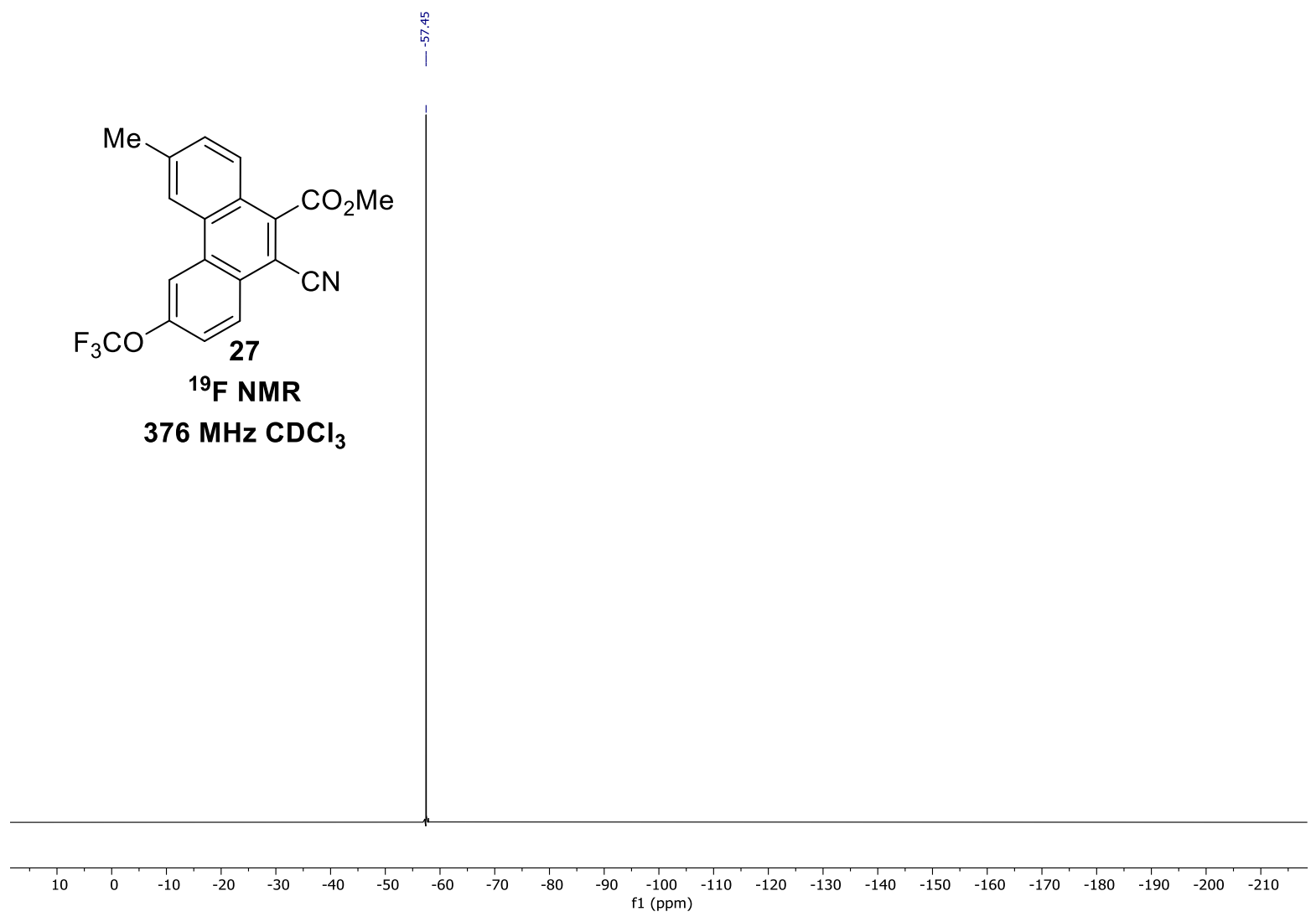



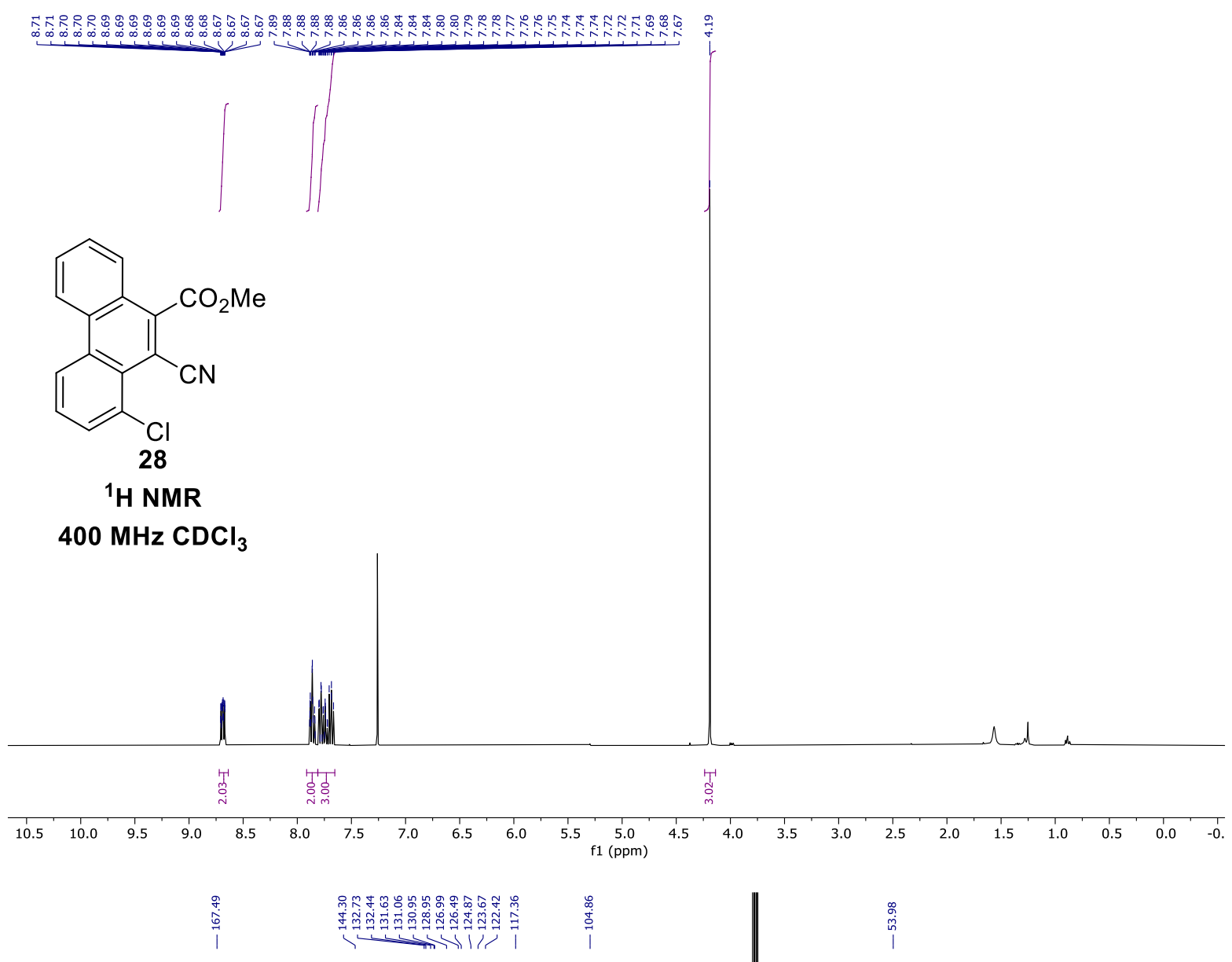<smiles>CC(=O)c1c(C#N)c2c(Cl)cccc2c2ccccc12</smiles>

28

${ }^{13} \mathrm{C}$ NMR

$101 \mathrm{MHz} \mathrm{CDCl}_{3}$

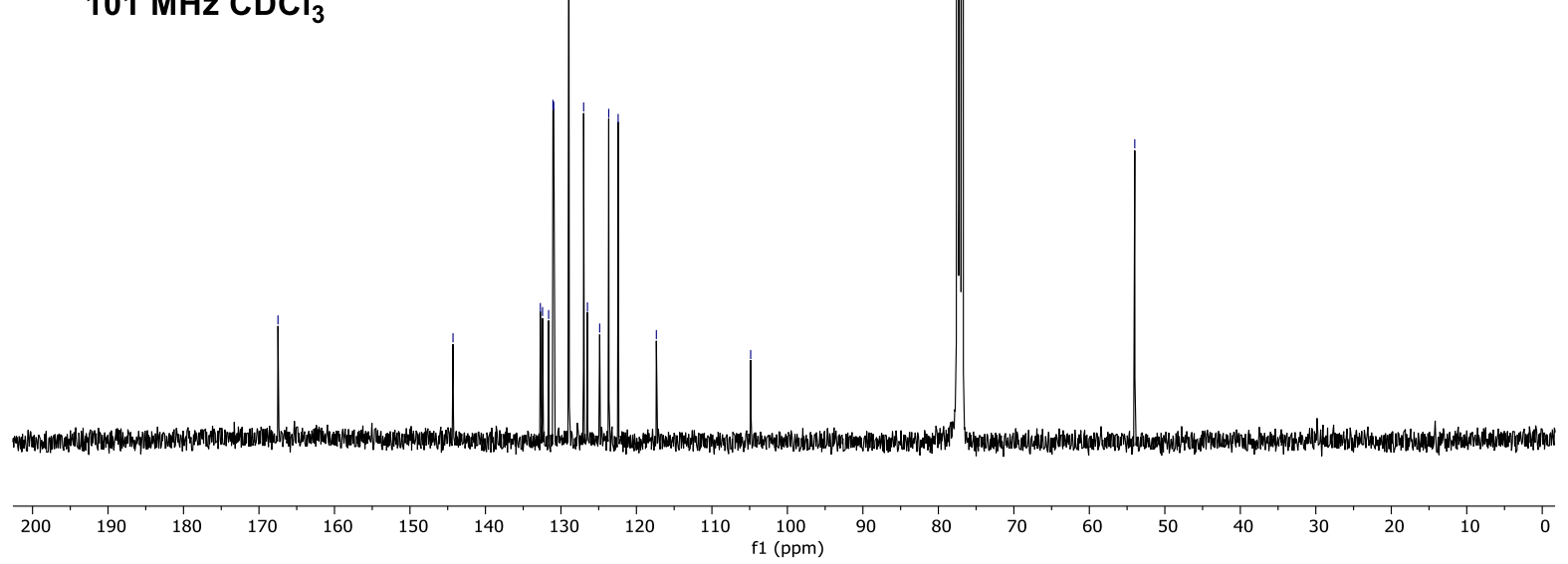



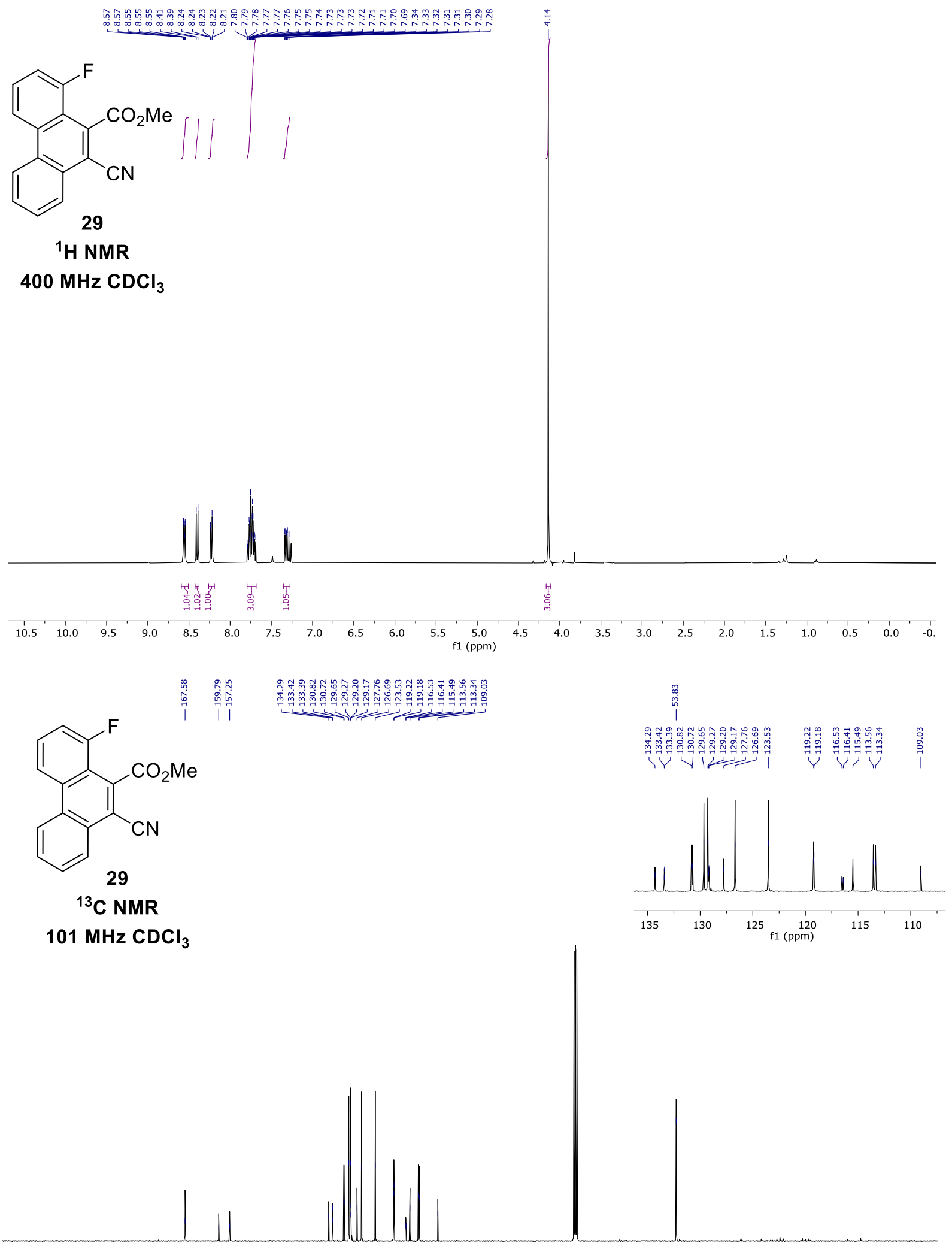

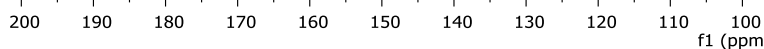


<smiles>CC(=O)c1c(C#N)c2ccccc2c2cccc(F)c12</smiles>

${ }^{19} \mathrm{~F}$ NMR

$376 \mathrm{MHz}^{\mathrm{CDCl}} \mathrm{S}_{3}$

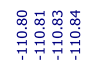

年

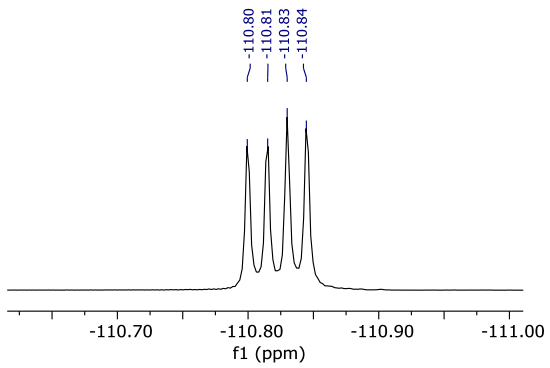

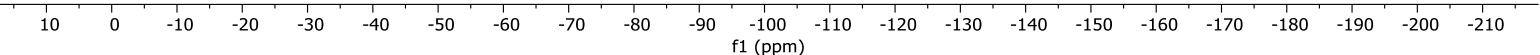




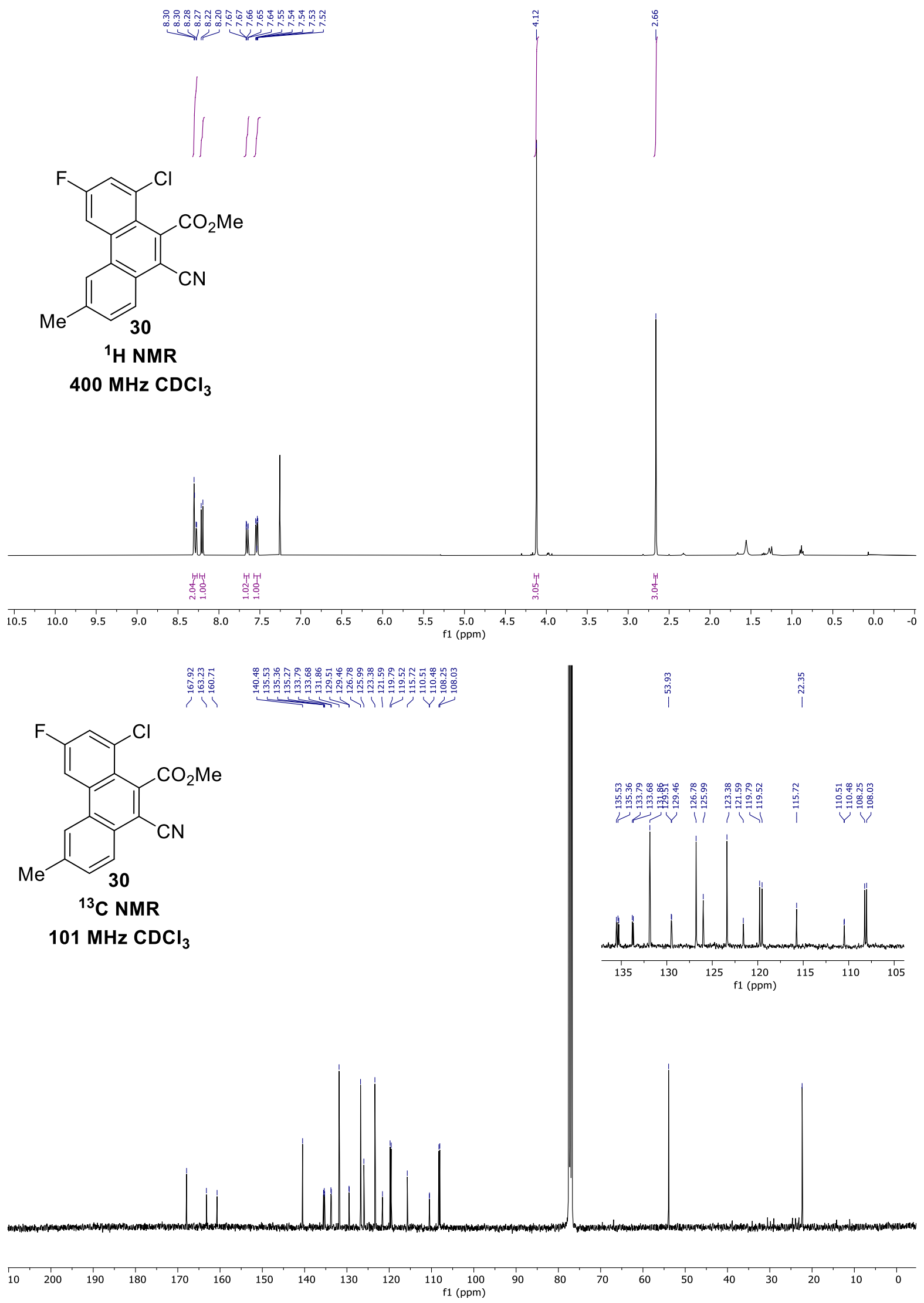




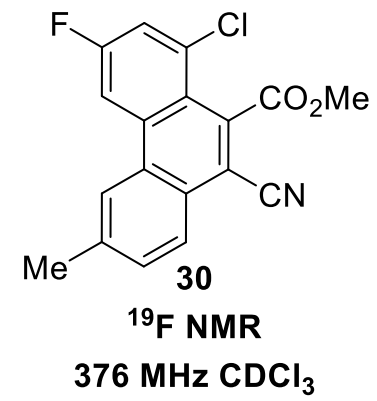

$\checkmark$

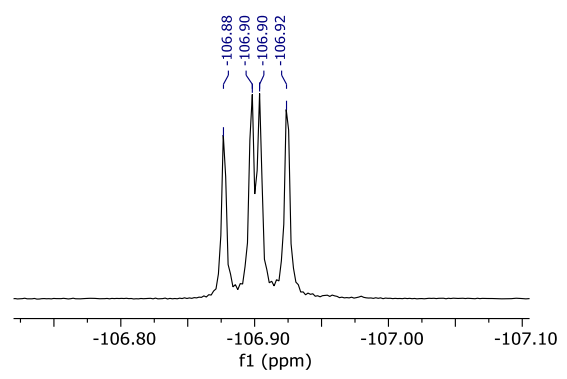

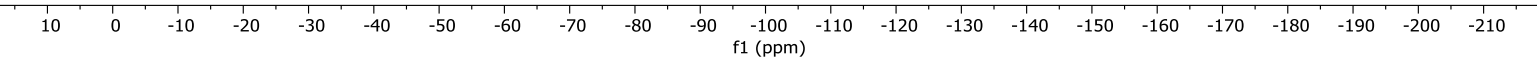




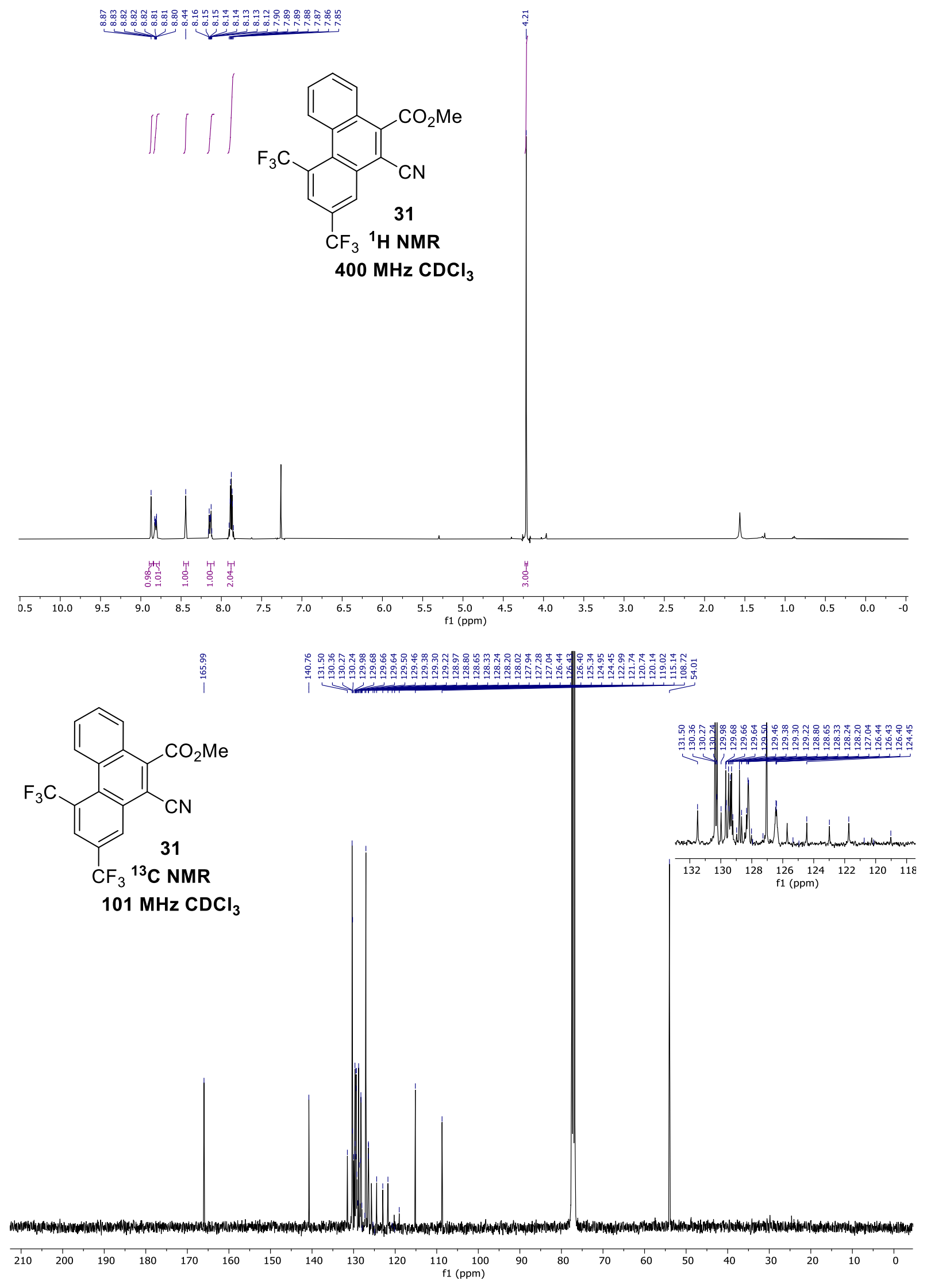




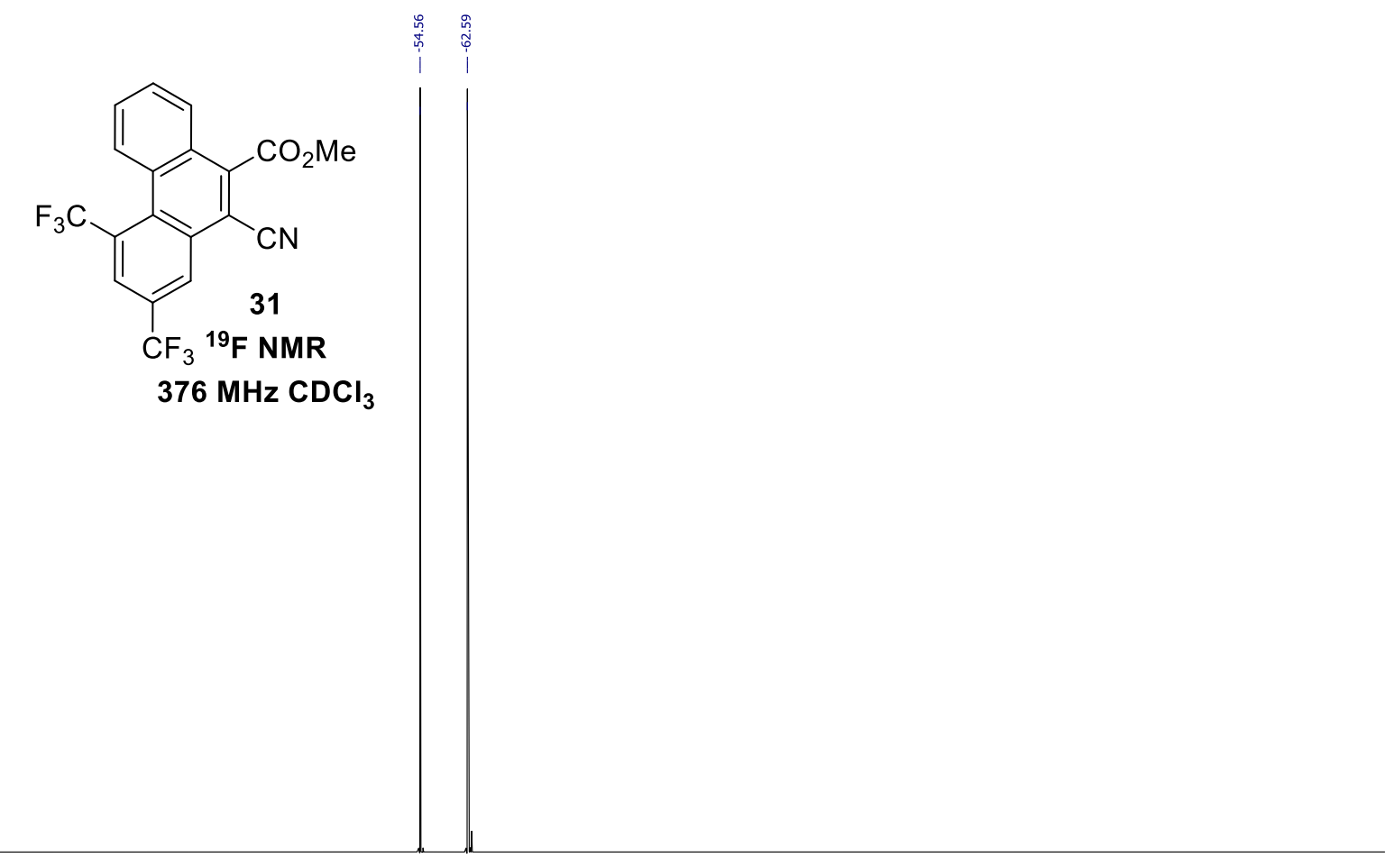

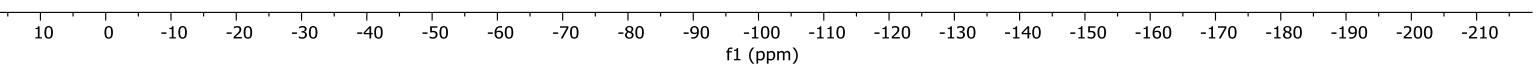




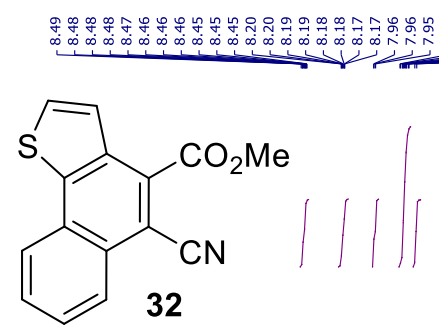

${ }^{1} \mathrm{H}$ NMR

$400 \mathrm{MHz} \mathrm{CDCl}_{3}$
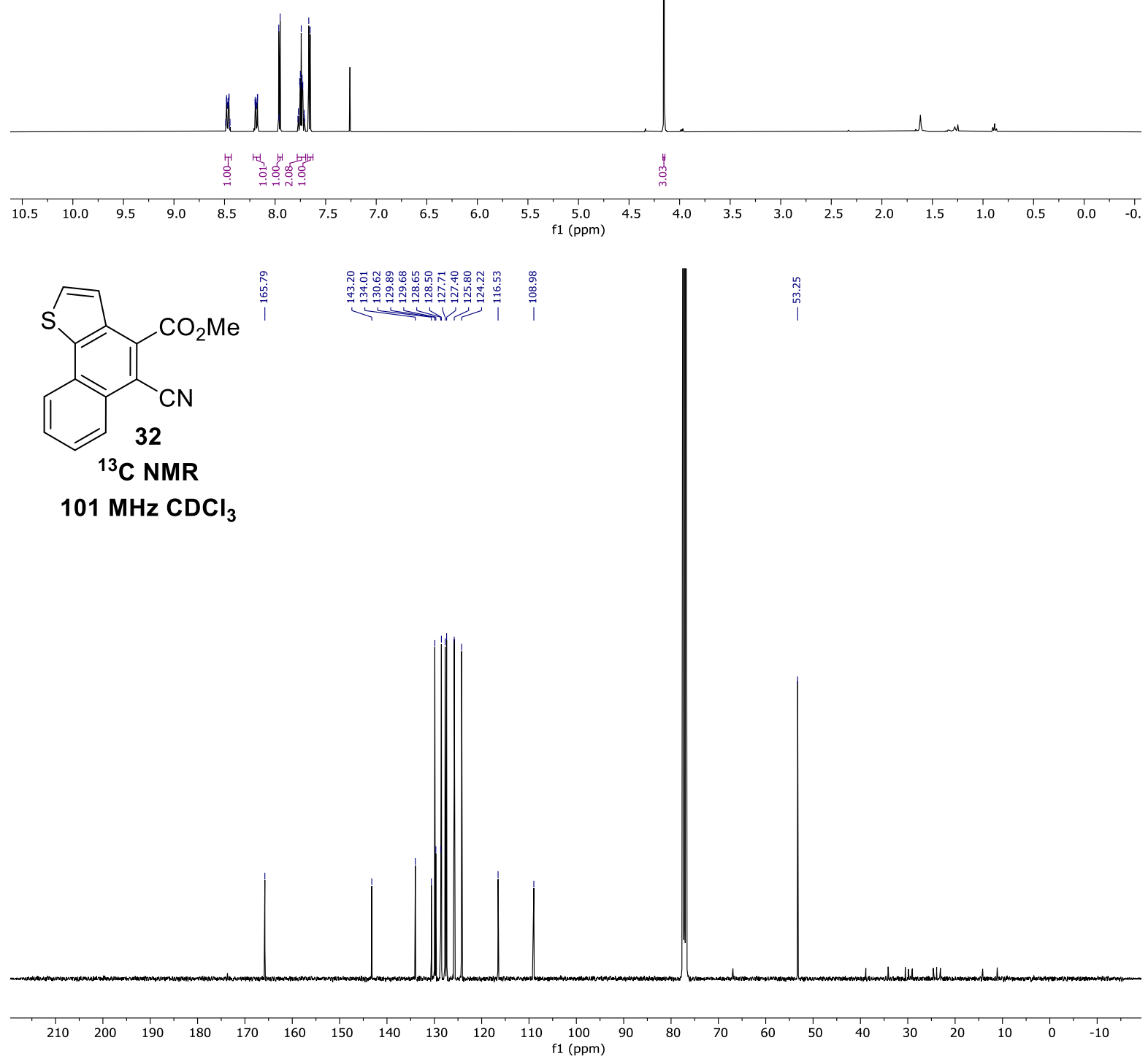

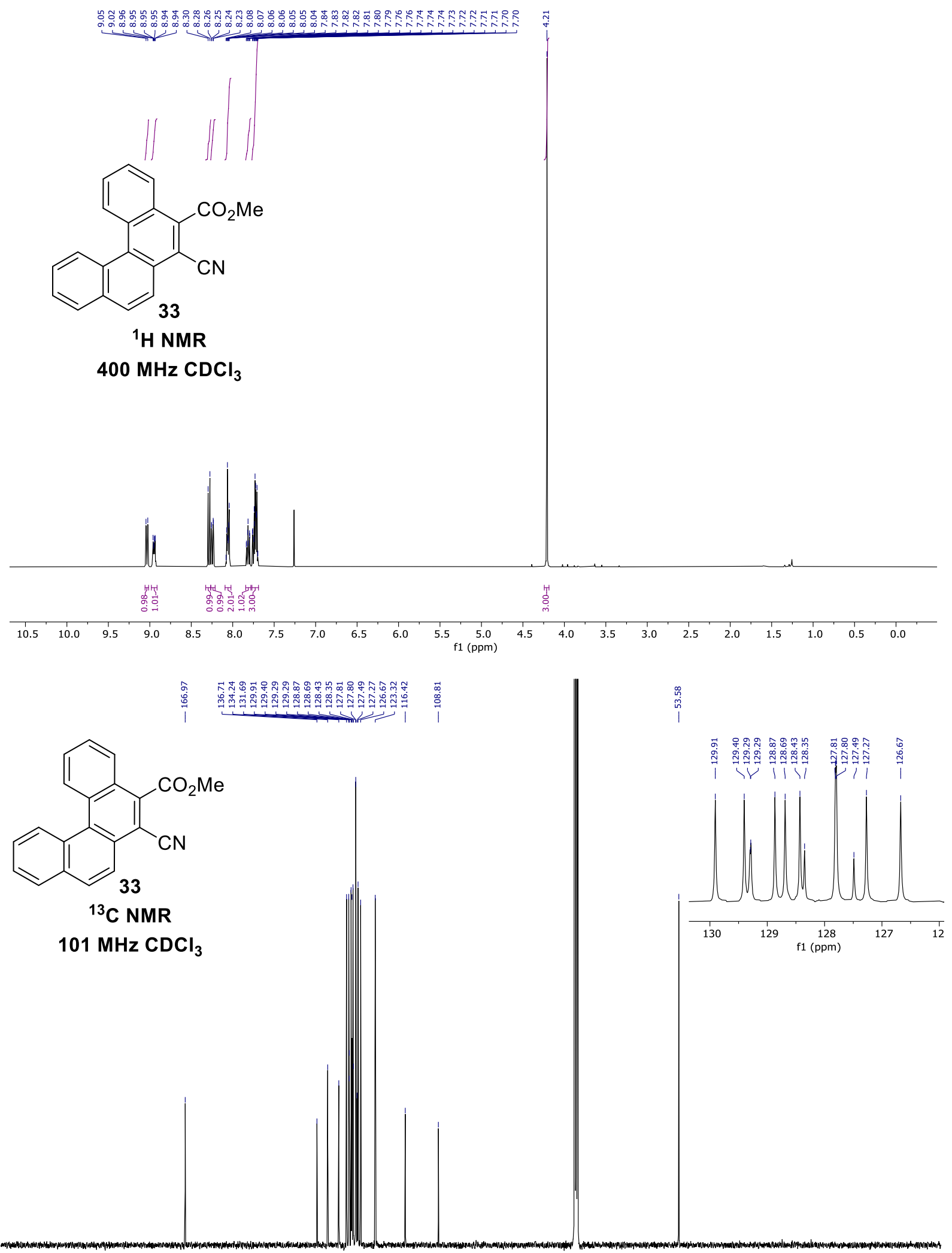

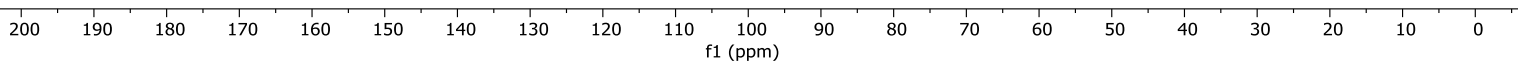



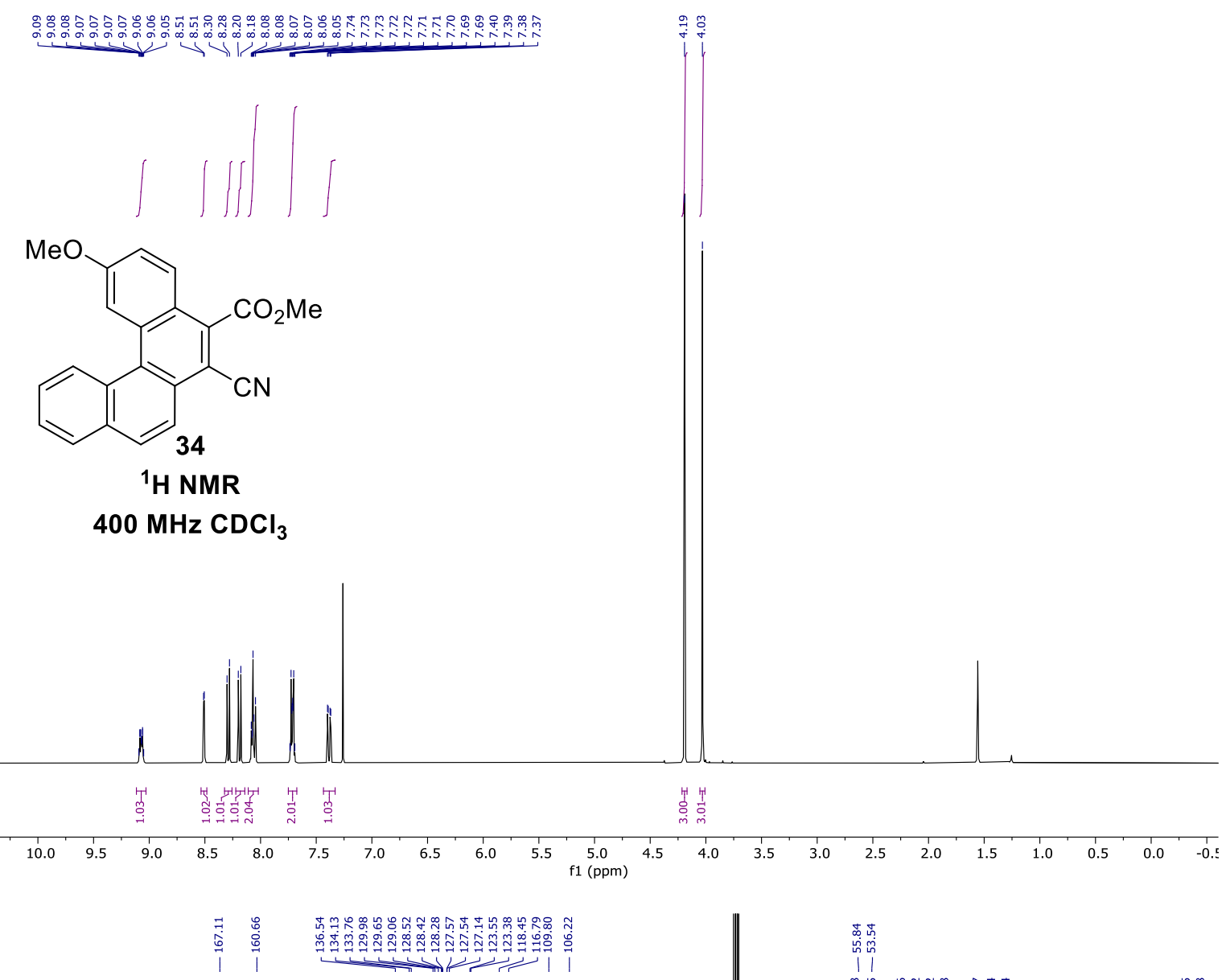<smiles>COc1ccc2c(C(C)=O)c(C#N)c3ccc4ccccc4c3c2c1</smiles>

${ }^{13} \mathrm{C}$ NMR $101 \mathrm{MHz}^{\mathrm{CDCl}}{ }_{3}$

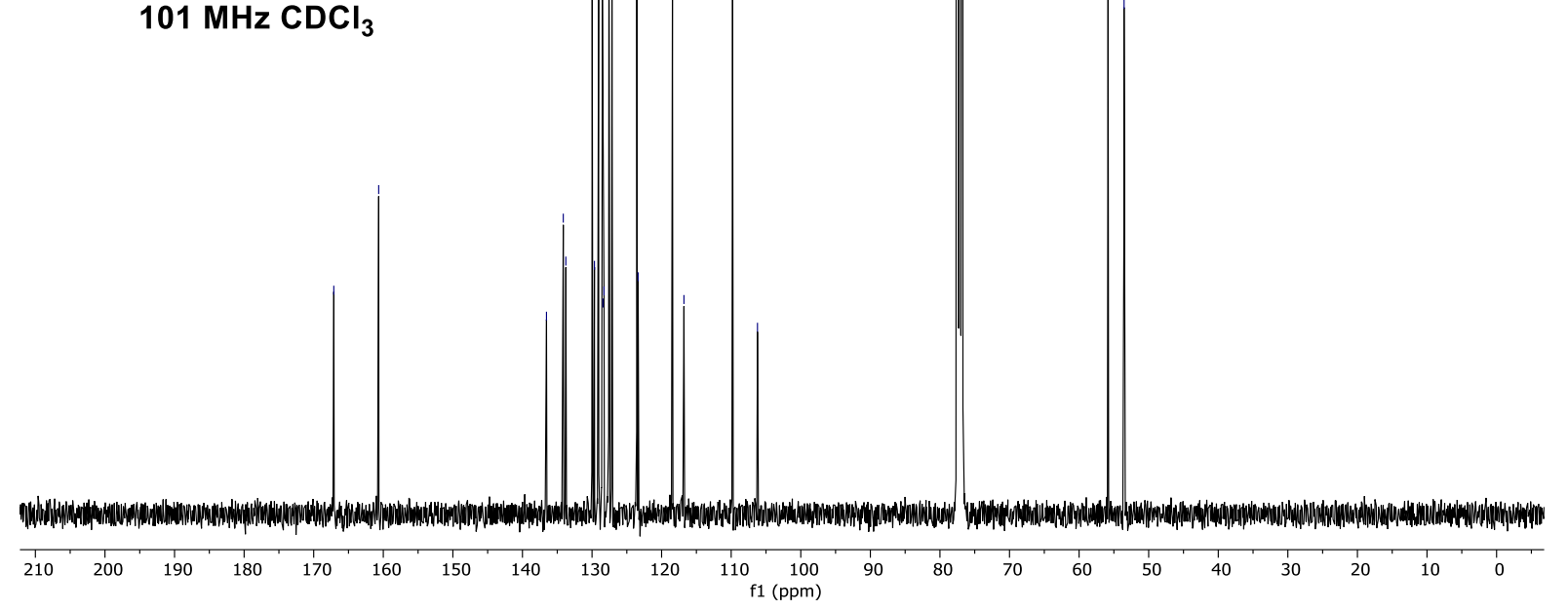




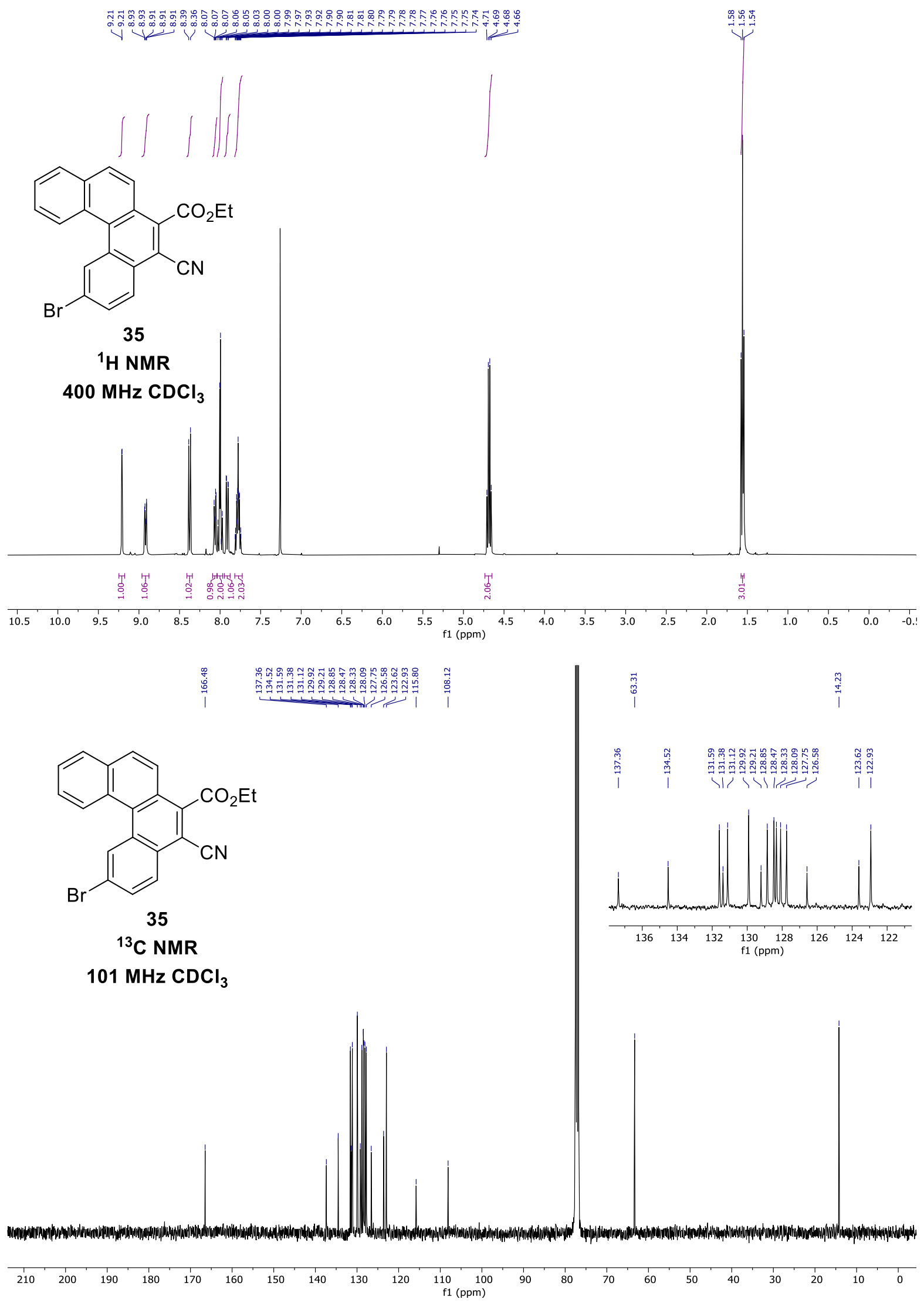




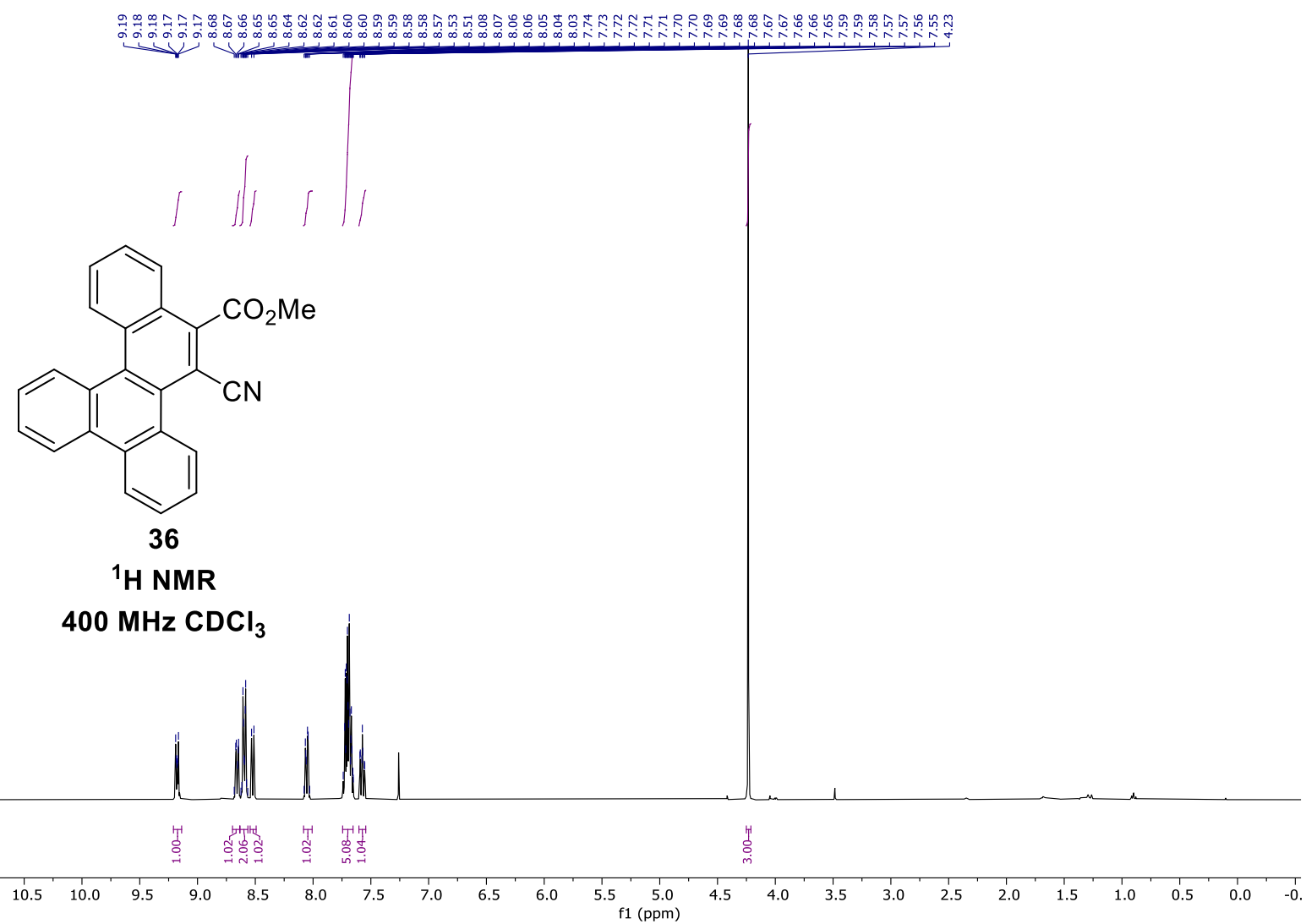

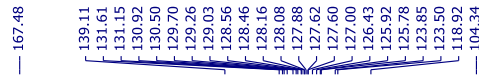<smiles>CC(=O)c1c(C#N)c2c3ccccc3c3ccccc3c2c2ccccc12</smiles>

36

${ }^{13} \mathrm{C}$ NMR $101 \mathrm{MHz} \mathrm{CDCl}_{3}$

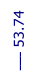

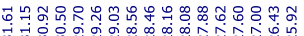
ติં (1)

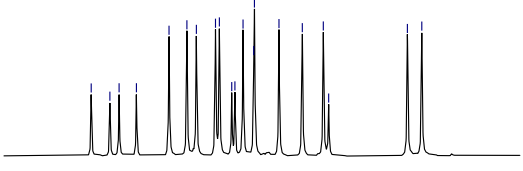

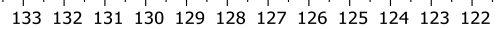
f1 (ppm)

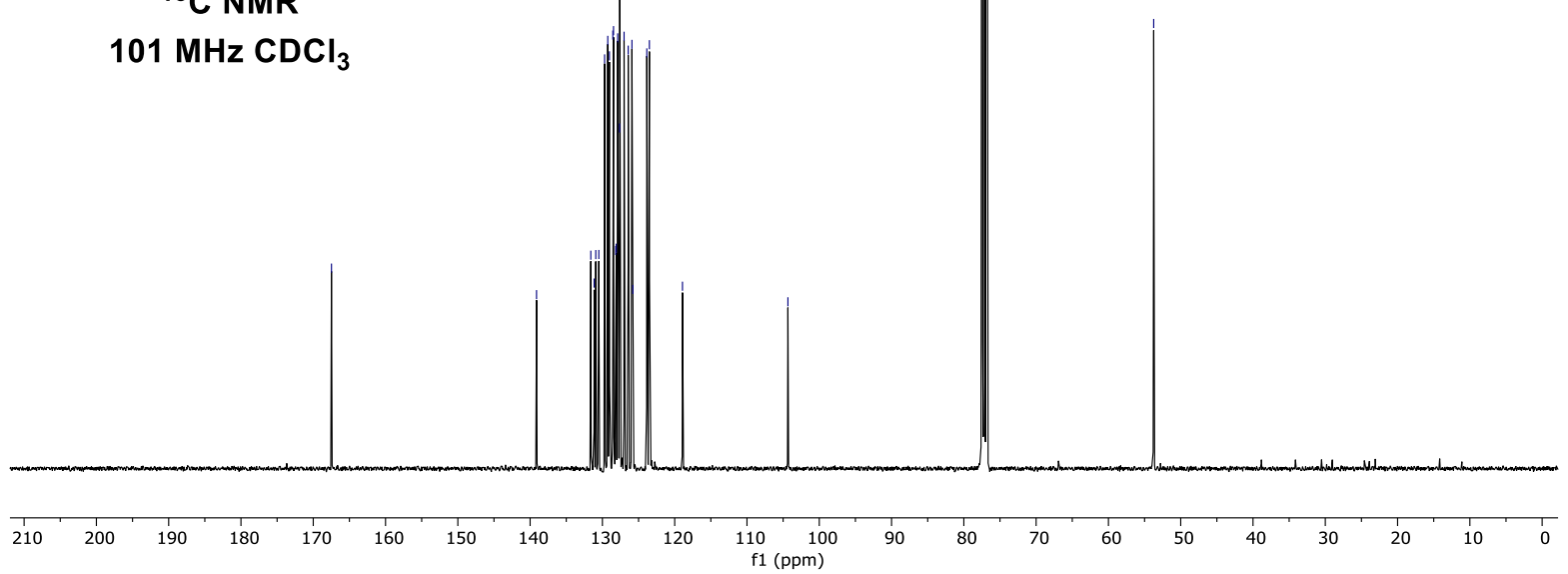



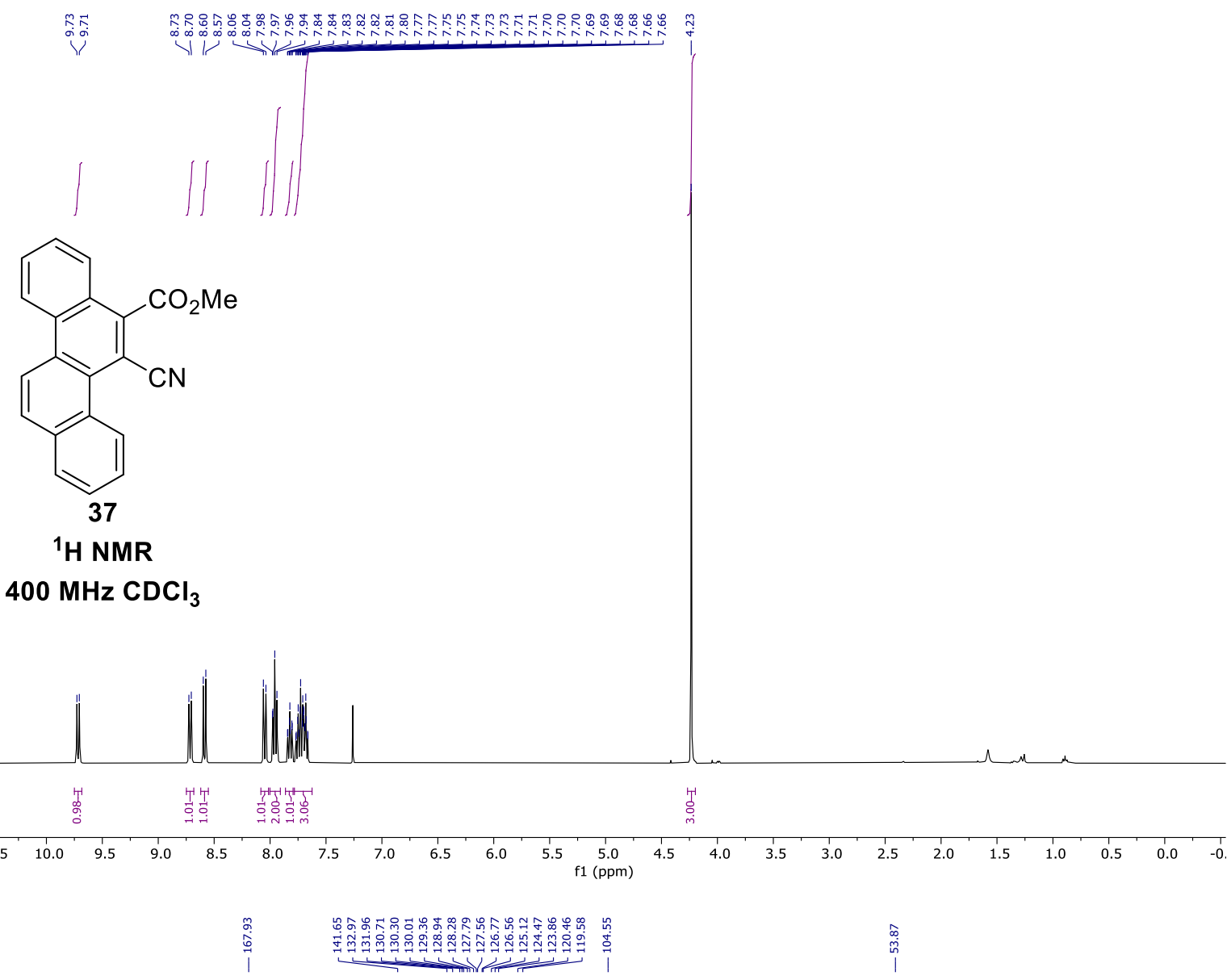<smiles>CC(=O)c1c(C#N)c2c3ccccc3ccc2c2ccccc12</smiles>

37

${ }^{13} \mathrm{C}$ NMR

$101 \mathrm{MHz}^{\mathrm{CDCl}_{3}}$
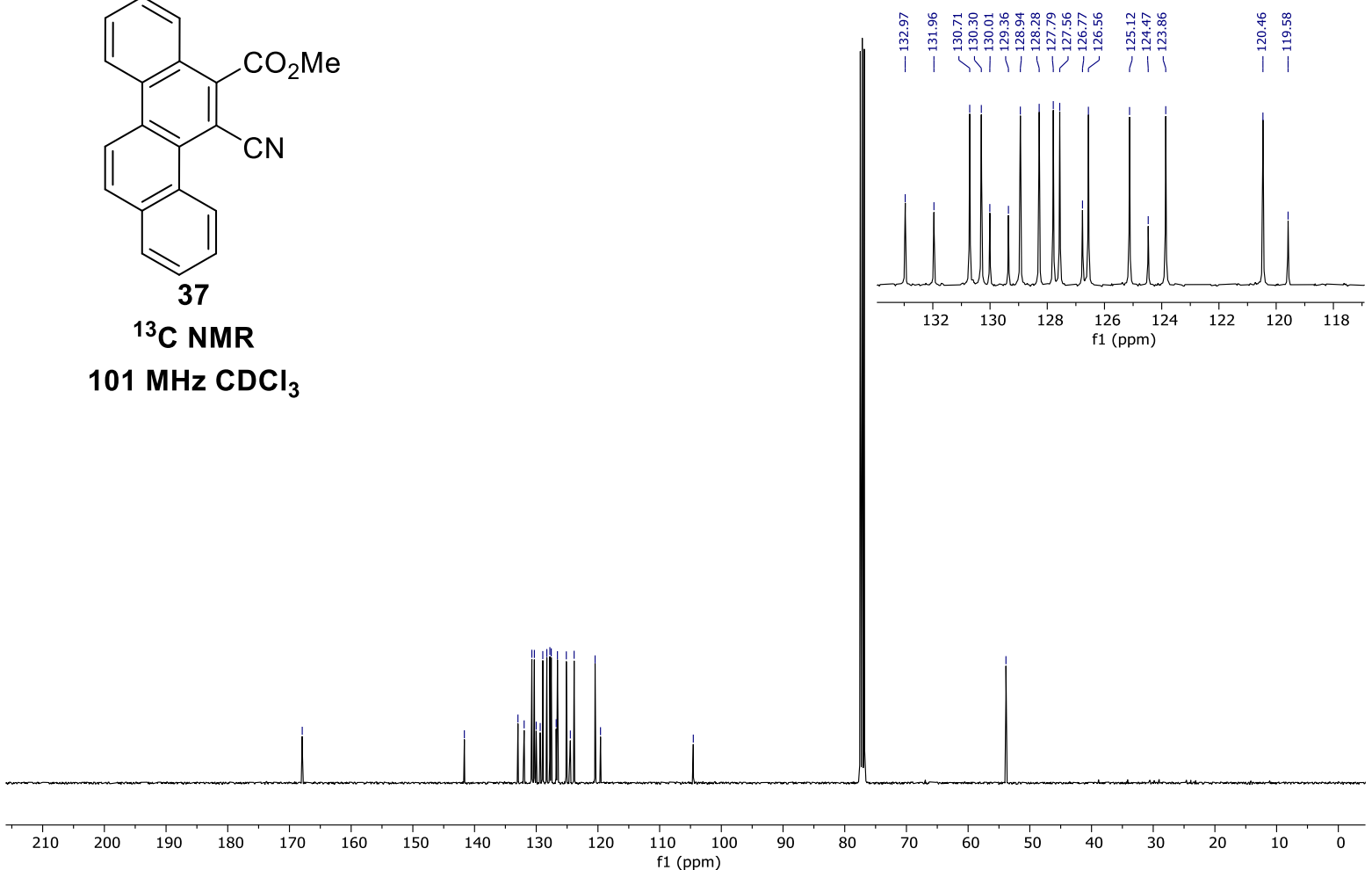


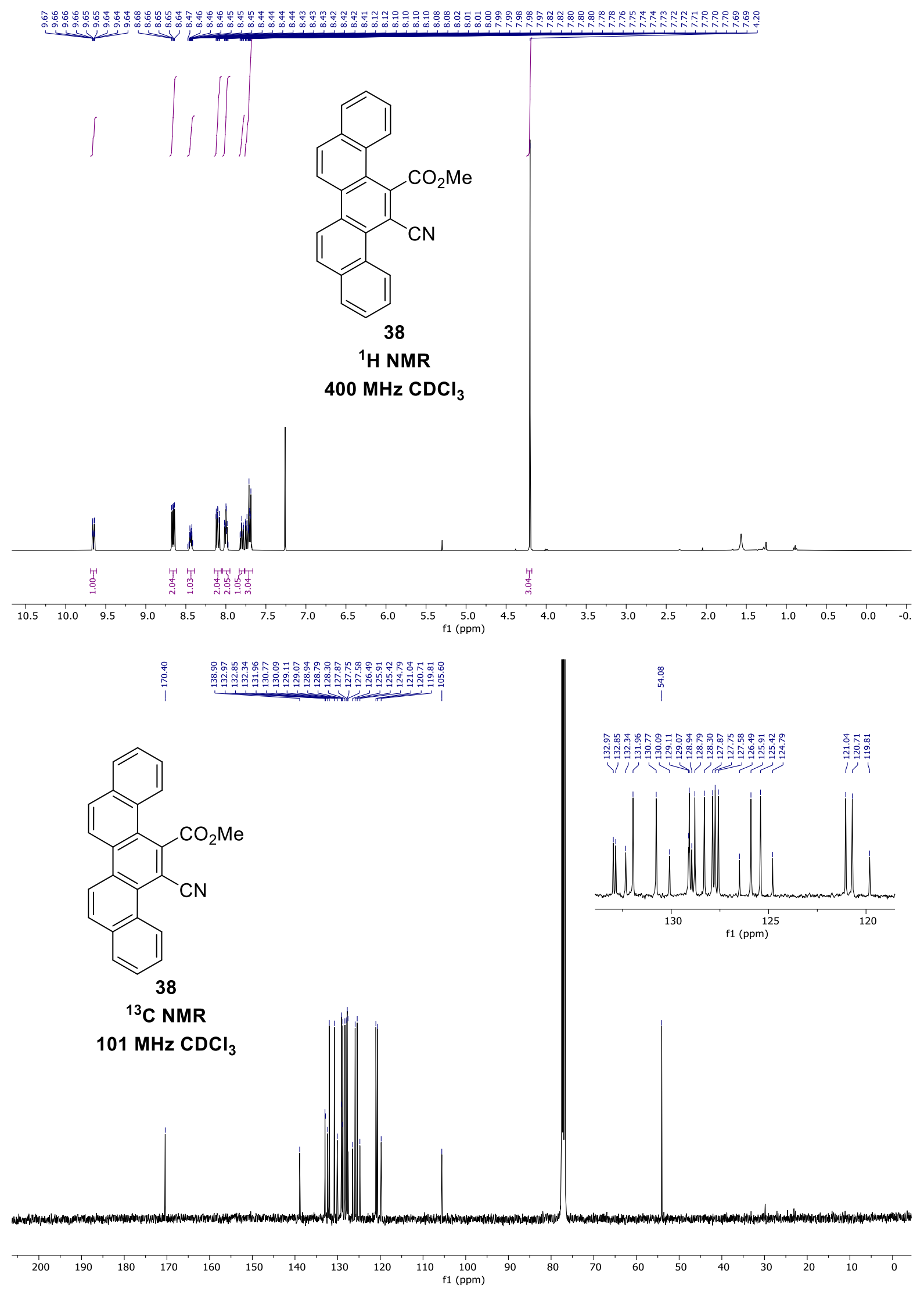




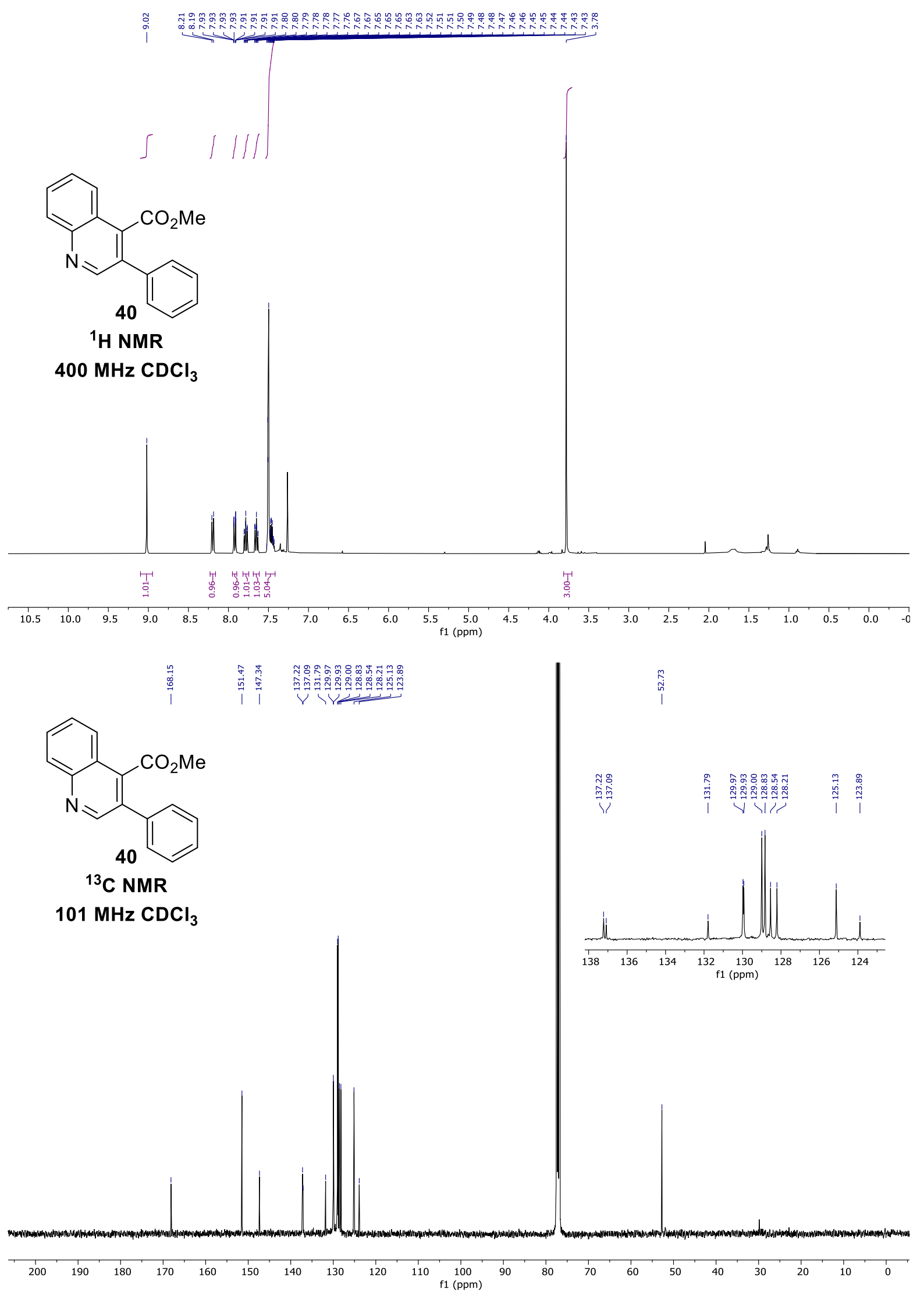

